\title{
Quantitative contrast-enhanced MRI of the microvasculature
}

Citation for published version (APA):

Oosterdorp, M. (2009). Quantitative contrast-enhanced MRI of the microvasculature. [Doctoral Thesis, Maastricht University]. Datawyse / Universitaire Pers Maastricht. https://doi.org/10.26481/dis.20100115mo

Document status and date:

Published: 01/01/2009

DOI:

10.26481/dis.20100115mo

Document Version:

Publisher's PDF, also known as Version of record

\section{Please check the document version of this publication:}

- A submitted manuscript is the version of the article upon submission and before peer-review. There can be important differences between the submitted version and the official published version of record.

People interested in the research are advised to contact the author for the final version of the publication, or visit the DOI to the publisher's website.

- The final author version and the galley proof are versions of the publication after peer review.

- The final published version features the final layout of the paper including the volume, issue and page numbers.

Link to publication

\footnotetext{
General rights rights.

- You may freely distribute the URL identifying the publication in the public portal. please follow below link for the End User Agreement:

www.umlib.nl/taverne-license

Take down policy

If you believe that this document breaches copyright please contact us at:

repository@maastrichtuniversity.nl

providing details and we will investigate your claim.
}

Copyright and moral rights for the publications made accessible in the public portal are retained by the authors and/or other copyright owners and it is a condition of accessing publications that users recognise and abide by the legal requirements associated with these

- Users may download and print one copy of any publication from the public portal for the purpose of private study or research.

- You may not further distribute the material or use it for any profit-making activity or commercial gain

If the publication is distributed under the terms of Article $25 \mathrm{fa}$ of the Dutch Copyright Act, indicated by the "Taverne" license above, 


\section{Quantitative contrast-enhanced MRI of the microvasculature}




\section{About the cover}

The cover shows a tropical house gecko (hemidactylus mabouia) on the ruins of the Great Mosque in the $16^{\text {th }}$ century town of Takwa on Manda Island (Lamu Archipelago, Kenya). The gecko's protective coloring, which makes it more difficult to distinguish the animal from its surroundings, represents the fact that the most interesting parts of an (MR) image can be hidden. Image quantification and post-processing may allow significant enhancement of these interesting features, as indicated on the back cover. Furthermore, the photograph represents the continuous balance between spatial resolution, acquisition time, signal intensity, and noise in MRI.

The work described in this thesis was funded by the BSIK program entitled Molecular imaging of ischemic heart disease (grant number 03033).

ISBN: 978-90-5278-902-6

(C) Copyright Marlies Oostendorp, Maastricht 2009 Printed by Datawyse / Universitaire Pers Maastricht. 


\title{
Quantitative contrast-enhanced MRI of the microvasculature
}

\author{
PROEFSCHRIFT \\ ter verkrijging van de graad van doctor aan de \\ Universiteit Maastricht, op gezag van de Rector \\ Magnificus, Prof. Mr. G.P.M.F. Mols, volgens het \\ besluit van het College van Decanen in het openbaar \\ te verdedigen op vrijdag 15 januari 2010 om 16:00 uur \\ door
}

\section{Marlies Oostendorp}

geboren op 11 oktober 1982 te Arnhem. 
Promotor

Prof. Dr. M.J. Post

Copromotor

Dr. Ir. W.H. Backes

Beoordelingscommissie

Prof. Dr. J.E. Wildberger (voorzitter)

Prof. Dr. A. Heerschap (Radboud Universiteit Nijmegen)

Prof. Dr. J.F.M. Smits

Prof. Dr. Ir. J.A.E. Spaan (Academisch Medisch Centrum Amsterdam)

Prof. Dr. F. Verhaegen

Financial support by the Netherlands Heart Foundation for the publication of this thesis is gratefully acknowledged.

Additional financial support was kindly provided by: Guerbet Nederland BV, Medtronic Bakken Research Center, Schering-Plough, and UNO Roestvaststaal BV. 


\section{Contents}

1 General introduction $\quad 1$

1.1 Magnetic resonance imaging 2

1.2 Contrast agents 6

$\begin{array}{ll}1.3 \text { Image quantification } & 7\end{array}$

1.4 Thesis objectives and outline 8

2 Vessel growth and function: depiction with contrast-enhanced MRI

2.1 Introduction 12

2.2 Mechanisms of neovascularization 12

2.3 Morphological imaging 14

2.4 Functional imaging $\quad 21$

2.5 Molecular imaging 29

2.6 Clinical considerations and future directions 33

2.7 Conclusions 35

I Tumor microvasculature $\quad 39$

3 Quantitative molecular MRI of tumor angiogenesis using cNGRlabeled paramagnetic quantum dots

3.1 Introduction 42

3.2 Methods 43

3.3 Results 49

3.4 Discussion 55

4 Gadolinium-labeled quantum dots for molecular MRI: $R_{1}$ versus $R_{2}$ mapping

4.1 Introduction 60

4.2 Methods 62

4.3 Results 65

$\begin{array}{lll}4.4 & \text { Discussion } & 69\end{array}$ 
5 Pharmacokinetics of contrast agents targeted to the tumor vas$\begin{array}{ll}\text { culature in molecular MRI } & 73\end{array}$

$\begin{array}{lll}5.1 & \text { Introduction } & 74\end{array}$

$\begin{array}{ll}5.2 \text { Methods } & 75\end{array}$

$\begin{array}{lll}5.3 & \text { Results } & 79\end{array}$

5.4 Discussion 84

6 Evaluation of MR vessel size imaging by two-photon laser scanning microscopy $\quad 89$

6.1 Introduction 90

$\begin{array}{ll}6.2 \text { Methods } & 91\end{array}$

6.3 Results 97

$\begin{array}{lll}6.4 & \text { Discussion } & 100\end{array}$

II Myocardial microvasculature 107

7 Molecular MRI of cardiac angiogenesis after acute myocardial infarction

$\begin{array}{lll}7.1 & \text { Introduction } & 110\end{array}$

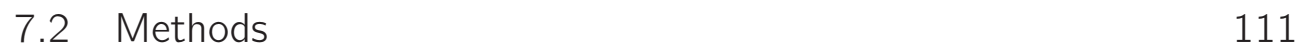

$\begin{array}{lll}7.3 & \text { Results } & 115\end{array}$

$\begin{array}{lll}7.4 & \text { Discussion } & 119\end{array}$

III Renal microvasculature $\quad 125$

$8 \mathrm{MRI}$ of renal oxygenation and function after normothermic ischemiareperfusion injury

8.1 Introduction 128

8.2 Methods 129

8.3 Results 132

$\begin{array}{lll}8.4 & \text { Discussion } & 137\end{array}$ 
9 General discussion $\quad 141$

9.1 Quantitative MRI in oncology 142

9.2 Quantitative MRI in cardiovascular disease 144

9.3 Quantitative MRI in nephrology 146

9.4 Translational challenges in molecular MRI 147

9.5 Towards clinical angiogenesis imaging 151

$\begin{array}{ll}9.6 \text { Conclusions } & 154\end{array}$

$\begin{array}{ll}\text { Appendices } & 155\end{array}$

A Pharmacokinetic modeling in DCE-MRI 157

B Magnetic effects of quantum dots 159

$\begin{array}{ll}\text { C IR and SE sensitivities } & 161\end{array}$

D Pharmacokinetic modeling in dynamic molecular MRI 163

$\begin{array}{ll}\text { Summary } & 189\end{array}$

$\begin{array}{ll}\text { Samenvatting } & 193\end{array}$

$\begin{array}{ll}\text { Dankwoord } & 197\end{array}$

$\begin{array}{ll}\text { Scientific publications } & 201\end{array}$

$\begin{array}{ll}\text { Curriculum vitae } & 203\end{array}$ 



\section{List of abbreviations}

\begin{tabular}{|c|c|}
\hline ADC & Apparent diffusion coefficient \\
\hline ARF & Acute renal failure \\
\hline ASL & Arterial spin labeling \\
\hline$B_{T P L S M}$ & Vessel branching by TPLSM \\
\hline BOLD & Blood oygen level dependent \\
\hline CA & Contrast agent \\
\hline CE-MRA & Contrast-enhanced MRA \\
\hline CEST & Chemical exchange saturation transfer \\
\hline cNGR & Cyclic Asn-Gly-Arg peptide \\
\hline CNR & Contrast-to-noise ratio \\
\hline cRGD & Cyclic Arg-Gly-Asp peptide \\
\hline $\mathrm{C} \top$ & Computed tomography \\
\hline DCE-MRI & Dynamic contrast-enhanced MRI \\
\hline DWI & Diffusion weighted imaging \\
\hline EC & Endothelial cell \\
\hline$E C G$ & Electrocardiogram \\
\hline ECM & Extracellular matrix \\
\hline ED & End-diastolic \\
\hline EES & Extracellular-extravascular space \\
\hline EF & Ejection fraction \\
\hline EPC & Endothelial progenitor cell \\
\hline ERS & Endocardial radial shortening \\
\hline ES & End-systolic \\
\hline ESI-MS & Electrospray ionization mass spectrometry \\
\hline $\mathrm{fBV} \bigvee_{\mathrm{MRI}}$ & Fractional blood volume by MRI \\
\hline FDG & Fluorodeoxyglucose \\
\hline FITC & Fluorescein isothiocyanate \\
\hline FLAPS & Fast low angle positive contrast steady state free precession \\
\hline FLASH & Fast low angle shot \\
\hline FOV & Field of view \\
\hline $\mathrm{NV}_{\mathrm{TPLSM}}$ & Fractional vessel volume by TPLSM \\
\hline Gd-DTPA & Gadolinium diethylenetriamine pentaacetic acid \\
\hline HBSS & Hanks balanced salt solution \\
\hline Hct & Hematocrit \\
\hline $\mathrm{I} / \mathrm{R}$ & Ischemia-reperfusion \\
\hline ICP-MS & Inductively coupled plasma mass spectrometry \\
\hline IR & Inversion recovery \\
\hline LAD & Left anterior descending coronary artery \\
\hline
\end{tabular}




\section{LIST OF ABBREVIATIONS}

$\begin{array}{ll}\text { LV } & \text { Left ventricle } \\ \text { MGE } & \text { Multi-echo gradient echo } \\ \text { MI } & \text { Myocardial infarction } \\ \text { MMP } & \text { Matrix metalloproteinase } \\ \text { MRA } & \text { Magnetic resonance angiography } \\ \text { MRI } & \text { Magnetic resonance imaging } \\ \text { MSME } & \text { Multi-slice multi-echo spin echo } \\ \text { MVD } & \text { Microvessel density } \\ \text { MVLTPLSM } & \text { Microvessel length by TPLSM } \\ \text { NA } & \text { Not applicable/available } \\ \text { NSF } & \text { Nephrogenic systemic fibrosis } \\ \text { OCT } & \text { Optimal cutting temperature } \\ \text { Pvox } & \text { Percentage of enhanced voxels } \\ \text { PAOD } & \text { Peripheral arterial occlusive disease } \\ \text { PAS } & \text { Periodic acid - Schiff } \\ \text { PCR } & \text { Polymerase chain reaction } \\ \text { PET } & \text { Positron emission tomography } \\ \text { PFC } & \text { Perfluorocarbon } \\ \text { PMT } & \text { Photomultiplier tube } \\ \text { PQDs } & \text { Paramagnetic QDs } \\ \text { QC } & \text { Quantitative contrast } \\ \text { QD } & \text { Quantum dot } \\ \text { RTPLSM } & \text { Vessel radius by TPLSM } \\ \text { RF } & \text { Radio frequency } \\ \text { RIMRI } & \text { Vessel radius index by MRI } \\ \text { ROI } & \text { Region of interest } \\ \text { S.C. } & \text { Subcutaneous } \\ \text { SE } & \text { Spin echo } \\ \text { SGM } & \text { Susceptibility gradient mapping } \\ \text { SNR } & \text { Signal-to-noise ratio } \\ \text { SPECT } & \text { Single photon emission computed tomography } \\ \text { TE } & \text { Echo time } \\ \text { TI } & \text { Inversion time } \\ \text { TNF } \alpha & \text { Tumor necrosis factor } \alpha \\ \text { TPLSM } & \text { Two-photon laser scanning microscopy } \\ \text { TR } & \text { Repetition time } \\ \text { USPIO } & \text { Ultrasmall superparamagnetic iron oxide particle } \\ \text { VCAM-1 } & \text { Vascular cell adhesion molecule 1 } \\ \text { VEGF } & \text { Vascular endothelial growth factor } \\ \text { VIF } & \text { Vascular input function } \\ \text { VSI } & \text { Vessel size imaging } \\ \text { XRA } & \text { X-ray angiography } \\ & \end{array}$




\section{1 \\ General introduction}

Angiogenesis is the growth of new blood vessels via sprouting from pre-existing vessels. It plays a relatively minor role in the healthy human adult and is only involved in wound healing and in the female reproductive cycle. In contrast, angiogenesis plays an important role in many common diseases $[1,2]$. Pathological angiogenesis can be divided in two categories based on its relative effect on disease development and patient prognosis, which can be either negative or positive (Figure 1.1). In this thesis one disease of each category will be investigated, namely tumor angiogenesis and angiogenesis after myocardial ischemia, respectively.

In cancer, angiogenesis supports tumor development by providing the growing tumor with its own blood supply. In addition, the rapid vessel growth contributes to the formation of metastasis, as tumor cells may spread to other parts of the body via the typically hyperpermeable blood vessels. The level of angiogenic activity was shown to be related to tumor malignancy and poor patient prognosis $[3,4]$. Consequently, there is a continuous effort to develop anti-angiogenic treatments, which can be given as single therapy or adjunctive to traditional radiation or chemotherapy.

In the heart, angiogenesis provides a natural repair mechanism to restore flow to ischemic tissue after myocardial infarction, and is a positive determinant of postinfarct remodeling and patient prognosis $[2,5]$. In this case, investigations are aimed at developing pro-angiogenic therapies in order to reduce or prevent myocardial damage after an infarction.

For either anti- or pro-angiogenic therapies, their success rate in clinical trials depends on reliable in vivo detection of angiogenic activity and definition of suitable clinical end-points. Furthermore, measurement of angiogenic activity may allow early evaluation of treatment response, as microvascular changes are expected to precede macrostructural changes, which can be detected using traditional anatomical imaging techniques.

Currently, estimation of tissue angiogenic activity is only possible via immunohistochemical determination of the microvessel density in biopsy samples. This significantly hampers longitudinal evaluation, is prone to tissue sampling errors, and is discomforting for patients. Consequently, novel methods need to be developed to detect angiogenic activity non-invasively in vivo. 
Magnetic resonance imaging (MRI) is one of the most versatile non-invasive imaging modalities available today. Clinical advantages are a superior spatial resolution and soft tissue contrast, and the absence of ionizing radiation. In addition, images can be obtained in any oblique direction with large anatomical coverage (Figure 1.1). It can detect blood vessels and vessel development at a morphological, functional, and recently also at a molecular level, as will be explained in more detail in Chapter 2. MRI is therefore the method of choice to develop clinically relevant methods to detect angiogenic activity in vivo.

\subsection{Magnetic resonance imaging}

This section is meant as a short introduction to the physical basics of MRI for the novice and can be skipped by the more experienced reader. For more detailed information on nuclear magnetic resonance in general, and more specifically on magnetic resonance imaging, the reader is referred to excellent text books by Levitt [14] and Haacke [15], respectively.

Magnetic resonance is based on the quantum mechanical property of atomic nuclei called spin. Loosely speaking, the spin of a nucleus indicates that the nucleus behaves as if it is spinning around, thereby generating a small magnetic moment. Not all nuclei have a spin, but the most abundant one, the hydrogen nucleus or proton, does. As tissues contain many protons in the form of water molecules (the average body water content is approximately $60 \%$ and water has a 110 molar proton concentration), it is the nucleus of choice for in vivo MRI.

Protons are so-called spin- $1 / 2$ nuclei, which means that there are two allowed spin quantum states, called spin-up and spin-down. When placed inside an external magnetic field, the small nuclear magnetic moments align either with or against the main magnetic field. As the spin-up state is slightly more populated than the spindown state, this generates a net overall magnetization $M_{0}$ that is aligned parallel to the main magnetic field $B_{0}$ (Figure 1.2). The relative population of the two

Figure 1.1 (facing page): Whole body MR image highlighting several diseases where angiogenesis plays either a positive or negative role. On the positive side, an increased blood supply could minimize neuronal damage in the brain. Furthermore, in the heart or in the legs, angiogenic activity provides a natural relief mechanism to protect the tissue against ischemia. On the negative side, angiogenesis can result in abnormal blood vessel growth in the eye in diabetes patients. In addition, angiogenesis supports tumor growth and metastatization, adds to the instability and growth of atherosclerotic lesions, and contributes to endometriosis development. Note that different MR techniques were applied to obtain the selected images, including anatomical imaging (whole body, eye, endometriosis, and atherosclerotic plaque), delayed contrast enhancement (heart), angiography (brain and lower extremities), and functional perfusion imaging (breast). Figure inspired by [6]; panels obtained from [7-13]. 


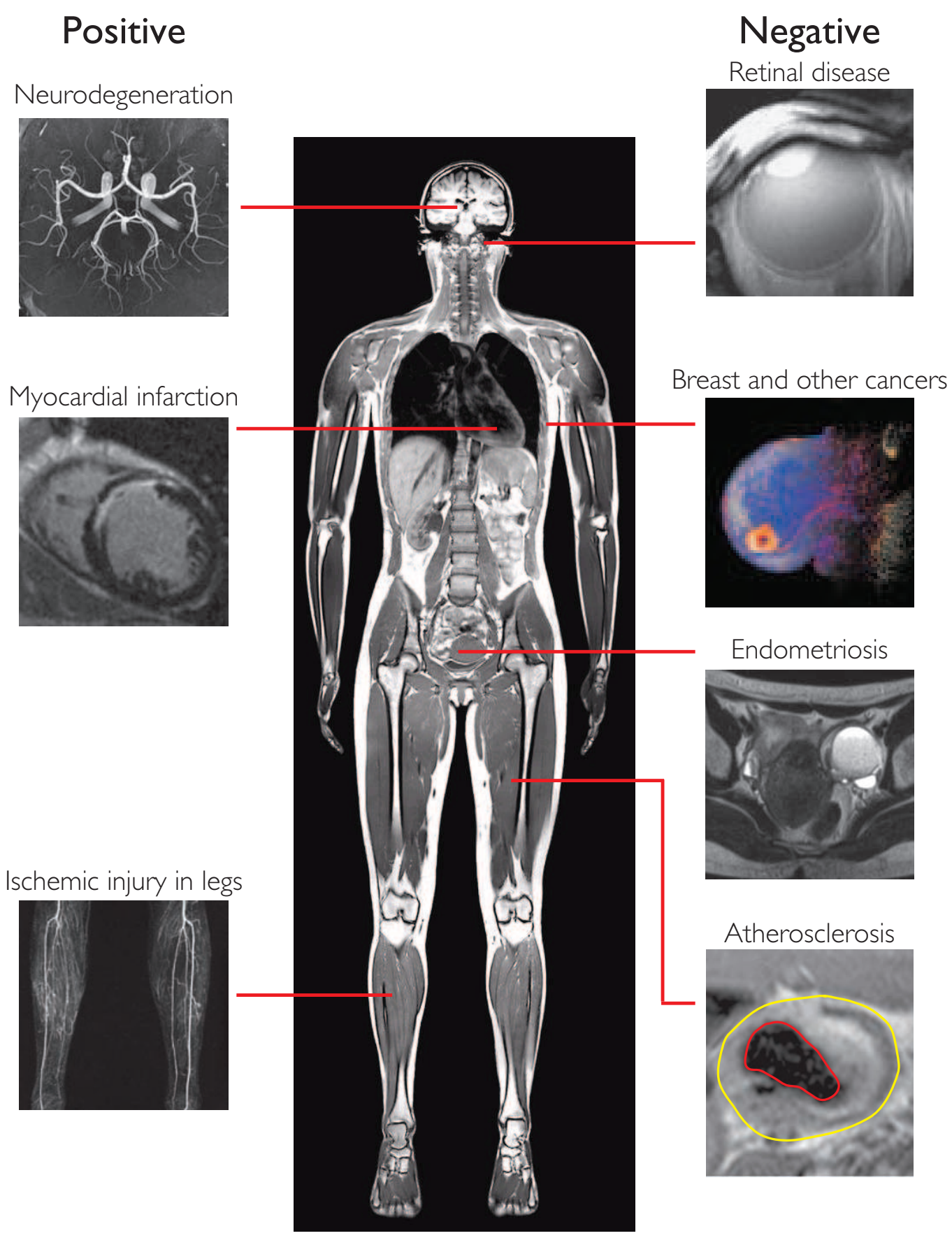




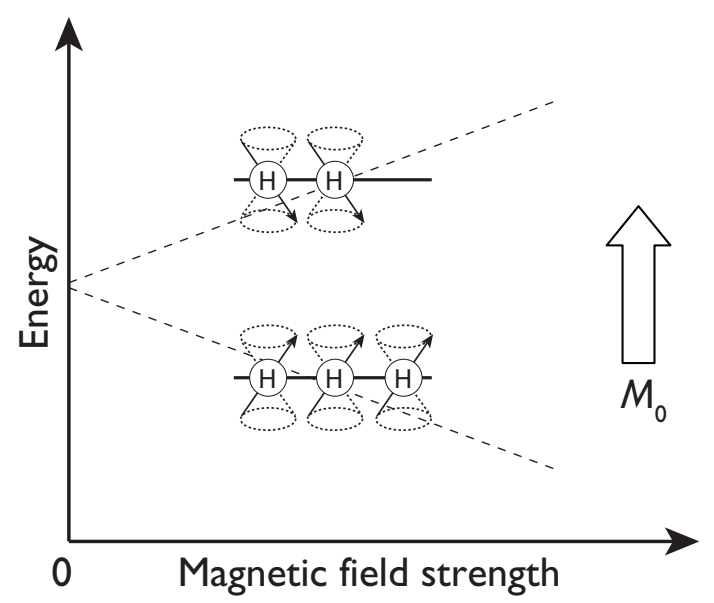

Figure 1.2: Energy difference between the spin-up and spin-down states of the hydrogen nucleus as a function of external magnetic field strength. Due to the slight population excess of the lower energy state (spin-up), a net magnetization is formed that is aligned parallel to the main magnetic field.

states is related to the energy difference between the states as described by the Boltzmann equation. In thermal equilibrium, this difference is relatively small, and, consequently, only few spins (in the order of 1 in every $10^{5}$ spins) contribute to the net magnetization. This explains the inherently low signal of MRI. However, the energy difference increases with increasing $B_{0}$ (Figure 1.2) and, consequently, the MRI signal also increases as function of $B_{0}$. This basically explains the general trend of imaging at higher field strengths.

A detectable MR signal can be obtained through various manipulations of $M_{0}$ using radio frequency (RF) pulses. Application of an RF pulse results in a "flip" of $M_{0}$ into the transverse plane, perpendicular to $B_{0}$ (Figure 1.3). The number of spins in the two quantum states is exactly equal and the spins have obtained the same phase (phase coherence). As the magnetization vector is now perpendicular to $B_{0}$, it experiences a torque, which causes it to precess around $B_{0}$ at the so-called Larmor frequency. This frequency depends on the type of nucleus and the strength of the magnetic field. For instance, the Larmor frequency of protons at field strengths of 1.5 and 7 Tesla is approximately 64 and $300 \mathrm{MHz}$, respectively. Note that the frequency of the applied RF pulse needs to be the same as the Larmor frequency to be able to flip the magnetization into the xy-plane. As a rotating magnetic moment induces an oscillating electrical current in an RF coil, the magnetization can be detected through its precession. Next, the resonance frequency can be obtained via a Fourier transformation of the oscillating signal.

Simultaneous with the precessional movement, relaxation processes bring the magnetization back to its equilibrium state, i.e. aligned parallel with $B_{0}$. Relaxation is divided into two components. First, the longitudinal magnetization will grow back to 

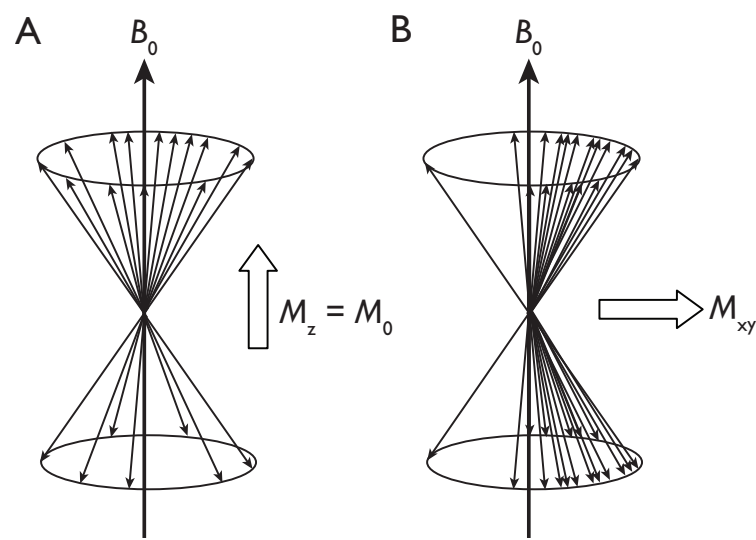

Figure 1.3: A) Schematic representation of the ensemble of spins in the equilibrium situation. There are slightly more spins in the spin-up than in the spin-down state and there is no phase coherence between the spins. Consequently, the net magnetization is oriented along the $z$-axis. Note that the $z$-direction is defined parallel to the main magnetic field and $M_{0}$ is therefore also called $M_{z}$. B) After an RF-pulse, the number of spins in the spin-up and spin-down states is exactly equal and the spins have predominantly obtained the same phase, resulting in a net magnetization in the xy-plane.

its steady-state value via spin-lattice interactions, with a characteristic time constant $T_{1}$. Second, transverse magnetization will decrease due to spin-spin interactions (dynamic field fluctuations experienced by the spins), resulting in a loss of the phase coherence between the individual spins in the ensemble. This process is governed by the time constant $T_{2}$. The loss of transverse magnetization can be accelerated by both microscopic and macroscopic field inhomogeneities. In this case, the relaxation process is referred to as apparent transverse relaxation, with a characteristic time constant $T_{2}^{*}$. All relaxation times are tissue-type dependent, whereas $T_{1}$-relaxation is also strongly dependent on magnetic field strength. Note that longitudinal and transverse relaxation are two independent processes. The lost xy-magnetization does not match the growth of z-magnetization. In fact, $T_{2}^{(*)}$-relaxation is always faster than $T_{1}$-relaxation.

Although the net magnetization $M_{0}$ can now be detected, it is unknown where the signal originated from in the body. Spatial encoding is achieved by applying magnetic field gradients. This causes the total magnetic field strength to change as a function of position in a linear way. As the Larmor frequency depends on field strength, the precession frequency is now directly related to the spatial position of the contributing spins. Another way of encoding spatial information is via the phase of the oscillating signal. Spatial encoding can be performed in one or multiple directions, after which the image is obtained by $2 \mathrm{D}$ or $3 \mathrm{D}$ Fourier transformation.

Image contrast is governed by multiple tissue-related and experimental parameters. The most important tissue parameters are proton density, and the relaxation times $T_{1}$ and $T_{2}^{(*)}$. Main experimental parameters include the repetition time, echo 


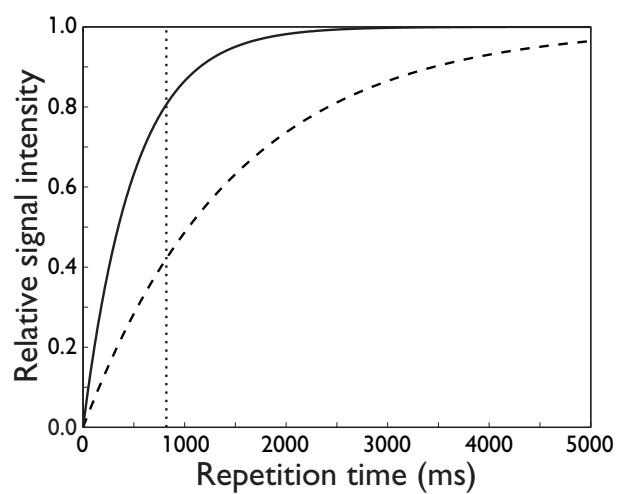

Figure 1.4: Signal intensity of a spin echo sequence as a function of repetition time (i.e. the time between two subsequent RF excitations) for two hypothetical tissues with a $T_{1}$ of $500 \mathrm{~ms}$ (solid line) and $1500 \mathrm{~ms}$ (dashed line) and equal proton density. The dotted vertical line shows the repetition time with the optimal image contrast between the tissues. Here, the tissue with the short $T_{1}$ has an almost two-fold higher signal intensity than the tissue with the longer $T_{1}$. At very long repetition times, the longitudinal magnetization has fully recovered for both tissues and the resulting image contrast will only depend on differences in proton density, and not on differences in $T_{1}$.

time, inversion time, and flip angle. As an example, Figure 1.4 shows how the image contrast of a standard spin echo sequence is related to $T_{1}$ and repetition time. For more information on the manipulation of tissue contrast, the reader is referred to the textbook of Haacke [15].

\subsection{Contrast agents}

Although the inherent soft tissue contrast of MR images is already superior compared with other non-invasive imaging modalities, it can be further enhanced using exogenous contrast agents. Traditionally, MR contrast agents can be categorized into positive and negative agents. Positive contrast agents cause a strong reduction in tissue $T_{1}$, which therefore appears brighter on $T_{1}$-weighted images. Clinically approved positive contrast agents are all based on low molecular weight chelates of the paramagnetic gadolinium ion, which has 7 unpaired electrons and therefore effectively reduces the relaxation times of nearby water protons. Negative contrast agents are usually based on particles of superparamagnetic iron oxide, which generate a strong reduction in the MRI signal due to shortening of the tissue $T_{2}$ and $T_{2}^{*}$.

The potency of a contrast agent to reduce relaxation times and hence to increase image contrast, is expressed using the relaxivity. For $T_{1}$-relaxation, the longitudinal relaxivity $r_{1}$ is defined as follows: 


\subsection{IMAGE QUANTIFICATION}

$$
\frac{1}{T_{1}}=\frac{1}{T_{1}^{0}}+r_{1} \cdot C
$$

Here, $T_{1}$ is the relaxation time after contrast agent administration, $T_{1}^{0}$ is the intrinsic tissue relaxation time (i.e. without contrast agent), and $C$ is the contrast agent concentration. A similar relationship holds for the transverse relaxivity $r_{2}$. Note that although relaxivity is a contrast agent specific parameter, it also depends on the external field strength, the macromolecular content (i.e. tissue and/or blood plasma [16]), and local conditions of water exchange [17]. The linear relationship of equation 1.1 is therefore not always guaranteed. For more details on the physicochemical mechanisms through which contrast agents reduce tissue relaxation, such as inner and outer sphere relaxation, the reader is referred to references $[15,18]$.

A novel class of contrast agents are the so-called targeted or molecular MRI contrast agents. These contrast agents are designed to detect biological processes in vivo at a cellular or molecular level. This is usually achieved via high-affinity ligands, such as antibodies, peptides or polysaccharides, coupled to the contrast agent. As these ligands are developed to bind specific biomarkers (e.g. receptors or enzymes) that are strongly upregulated during disease, the image contrast is only enhanced at the location of these biomarkers. One of the first molecular MRI examples was given by Sipkins et al., who used an antibody coupled to a gadolinium based contrast agent to selectively image angiogenic activity in tumors [19]. The development and, in particular, the application of a novel molecular MRI contrast agent to detect angiogenesis in tumors and in the heart are the main topics of this thesis.

\subsection{Image quantification}

Visual evaluation of MR images is an established tool in both clinical and research settings to detect abnormalities with great anatomical detail. However, unique additional information can often be obtained through image quantification. With respect to blood vessels and vessel development, quantitative MRI allows, for instance, measurement of vascular flow, vessel permeability, relative blood volume, vessel size, and tissue perfusion (see also Chapter 2). In the case of molecular MRI, quantification of tissue relaxation times may allow estimation of local contrast agent concentration and, consequently, the level of angiogenic activity. As outlined previously, this would be very beneficial for patient diagnosis and prognosis, and for the development of novel anti- and pro-angiogenic therapies for tumors and myocardial infarction, respectively.

Quantitative MRI has several advantages over structural MRI. First, it provides objective parameters that are independent of experimental parameters, such as repetition time, echo time, or flip angle. This allows better and easier comparison of results obtained from different subjects, recorded on different days, or using different 
equipment. This would be especially important for longitudinal and/or multicenter studies. Second, quantitative MRI often enhances only the relevant features of an image, for instance via reduction of noise and image artifacts. Third, there is no need for subjective image interpretation and images can be analyzed automatically. The downside, however, is that the data processing involved in quantitative MRI is often lengthy and can take up to several hours, depending on computational power, the complexity of the calculations, and the amount of data. Although quantitative analysis of MR images is currently not yet routine, it is starting to become available in dedicated clinical centers.

\subsection{Thesis objectives and outline}

The main goal of this thesis was to implement and apply a variety of quantitative contrast-enhanced MRI techniques to study microvascular properties and adaptations in murine models of cancer, myocardial infarction, and renal ischemia-reperfusion injury. In Chapter 2, a comprehensive overview is presented on the biological mechanisms of neovascularization and on the specific properties of the microvasculature that can be exploited by MRI. It describes the available MRI techniques that can be applied to study vessel growth and function, which provides the technical basis for the experimental studies described in Chapters $3-8$. The contents of these subsequent chapters are divided into three parts, each dealing with MR imaging of a different microvascular bed.

Part I focuses on quantitative MRI techniques to characterize the tumor microvasculature. Chapter 3 describes the development and application of a novel bimodal targeted contrast agent, which allowed specific imaging of the angiogenic tumor vasculature using in vivo molecular MRI and ex vivo two-photon laser scanning microscopy (TPLSM). The relaxation and contrast enhancing properties of the contrast agent are further investigated in Chapter 4. In Chapter 5, dynamic contrast-enhanced $\mathrm{MRI}$ of targeted molecular MRI contrast agents is described and a two-compartment model was developed to study their dynamic homing behavior and pharmacokinetics in tumor tissue. MR vessel size imaging using an iron oxide-based contrast agent is described in Chapter 6, which evaluates the validity of MR derived measures for the average tumor vessel diameter and fractional blood volume using a quantitative three-dimensional TPLSM approach.

Part II addresses the microvascular adaptations that occur in both the infarcted and remote cardiac regions after an acute myocardial infarction. Chapter 7 describes the use of the bimodal targeted contrast agent to selectively image neovascularization in the mouse heart using in vivo molecular MRI and ex vivo TPLSM.

Part III concentrates on microvascular damage due to ischemia-reperfusion injury in the kidney, which is, for instance, induced by renal transplantation. In Chapter 8, blood oxygen level dependent MRI and dynamic contrast-enhanced MRI are applied 


\subsection{THESIS OBJECTIVES AND OUTLINE}

to study intrarenal oxygenation and perfusion, respectively, up to 24 hours after ischemia-reperfusion injury.

This thesis is concluded by Chapter 9, which provides a general discussion on the previously described experimental studies. Main conclusions are drawn and potential future developments towards clinical translation of quantitative contrast-enhanced MRI of the microvasculature are discussed. 



\section{2 \\ Vessel growth and function: depiction with contrast-enhanced MRI}

Magnetic resonance imaging $(\mathrm{MRI})$ is a versatile, non-invasive diagnostic tool that can be applied to the entire human body, revealing morphological, functional, and metabolic information. Here, we review how MRI can visualize both the established and the developing vasculature with techniques involving intravenously administered contrast agents. In addition to macrovascular morphology and flow, $\mathrm{MRI}$ is able to exploit microvascular properties including vessel size distribution, hyperpermeability, flow heterogeneity and possibly also upregulation of endothelial biomarkers. For each MRI method, the basic principles, potential acquisition and interpretation pitfalls, solutions and applications are described. Furthermore, the discussion includes current shortcomings and the impact of future developments (e.g. higher magnetic field strength systems, targeted macromolecular contrast agents) on the visualization of blood vessel growth and function with contrast-enhanced MRI.

Radiology. 2009; 251: 317-35 


\subsection{Introduction}

Neovascularization is important for many physiologic processes, including embryonic development, wound healing and the menstrual cycle. However, there are also diseases where defective neovascularization is part of the condition and particularly the progression thereof [1]. The most prevalent diseases associated with abnormal vessel growth are cancer and atherosclerosis.

Currently, a number of therapeutic agents have been developed for cancer and cardiovascular disease treatment; these agents either inhibit (e.g. bevacizumab) or stimulate neovascularization, respectively $[20,21]$. However, to fully assess their therapeutic potential, non-invasive measurements are required that allow both diagnosis of the pathology as well as short and long-term evaluation of treatment response.

Of the available non-invasive imaging modalities, such as magnetic resonance imaging (MRI), computed tomography ( $\mathrm{CT}$ ), positron emission tomography (PET), and single photon emission computed tomography (SPECT), MRI may be the most powerful and versatile modality for imaging the vascular system, as it allows both morphological and functional characterization, possibly even within one session. Moreover, MRI is able to investigate vessel growth at different spatial and temporal scales, and is generally more sensitive to small vessel function than other modalities [22]. In addition, recent experimental developments demonstrate molecular characterization of neovascularization with MRI.

Here, the currently available MRI techniques to image the neovasculature are reviewed. Although several excellent papers on this topic were already published [23-27], the focus of this review will be on contrast-enhanced MRI methods as most new developments require contrast agent administration. Contrast-enhanced methods usually show a higher target-to-background contrast and facilitate fast imaging techniques, as compared with non-enhanced techniques such as time-of-flight angiography, phase contrast angiography, or arterial spin labeling. We will discuss the translation from in vivo animal imaging to the clinic and present clinical implementations. Also, we will address imaging of both angiogenesis and arteriogenesis associated with cardiovascular ischemic injury and show how techniques originally developed for oncological imaging appear valuable to cardiovascular problems.

\subsection{Mechanisms of neovascularization}

Neovascularization is generally considered to occur through one of three mechanisms $[28,29]$. First, vessels can be formed through vasculogenesis, which is the major process of vessel growth in the embryo $[1,28]$. During vasculogenesis, circulating endothelial progenitor cells (EPCs) differentiate into endothelial cells (ECs) that subsequently assemble into a vascular network [28]. Although vasculogene- 

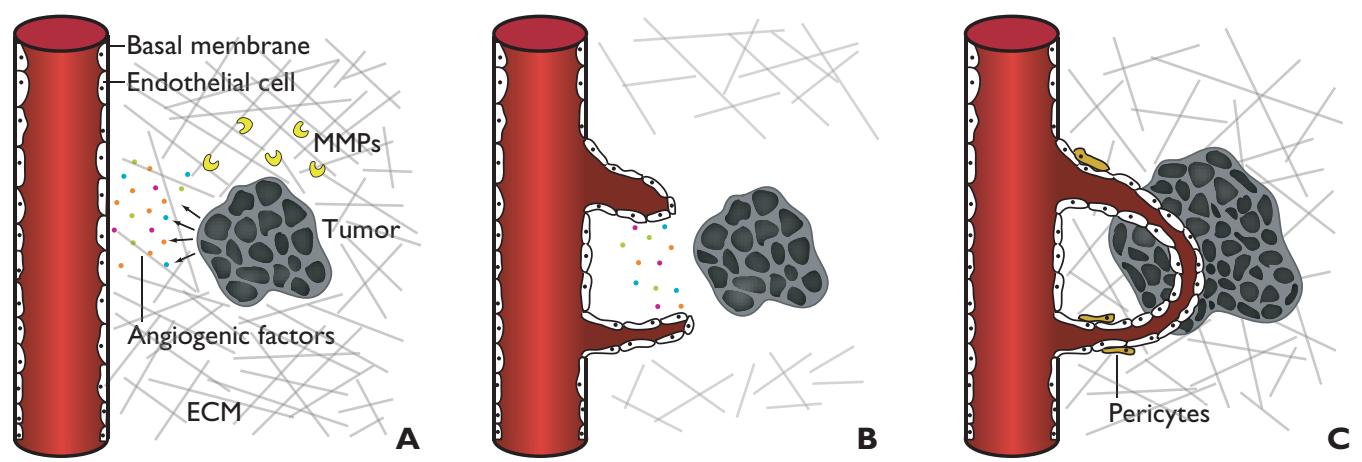

Figure 2.1: Schematic representation of the different steps of angiogenesis. A) A tumor triggers neovascularization by excreting angiogenic cytokines. B) Migration and proliferation of ECs towards the tumor. C) Formation of a new vessel lumen and vessel maturation. This final maturation step is not completed during tumor angiogenesis. ECM: extracellular matrix, MMPs: matrix metalloproteinases.

sis was previously thought to be a solely embryonic process, EPCs are also present in the adult circulation and can be mobilized from bone marrow by cytokines and growth factors, and during ischemic conditions [30]. EPCs contribute to postnatal neovascularization and maintenance of the vascular wall via incorporation into sites of vessel growth and differentiation into mature ECs [30-32]. Currently, promising results have been obtained with EPC-based therapies for cardiovascular ischemic diseases, showing improved left ventricular ejection fraction and myocardial perfusion, although the exact working mechanism and therapeutic potential of these therapies are still incompletely characterized [31].

Angiogenesis, the sprouting of vessels from pre-existing ones, is the second mechanism and is for instance involved in tumor neovascularization. In brief, angiogenesis is thought to be triggered by hypoxia, resulting in an increased production of various cytokines, of which vascular endothelial growth factor (VEGF) is the most important $[1,2,28,29]$. Potent endogenous inhibitors of VEGF are the Notch signaling pathway and Delta-like ligand $4[33,34]$. Tumor cells can independently induce vessel growth by excreting cytokines, even without being hypoxic (Figure 2.1) [35, 36]. Next, the basal membrane and extracellular matrix (ECM) are degraded and interendothelial cell contacts are loosened by matrix metalloproteinases (MMPs) and other proteases. EC migration and proliferation results in the formation of a new vessel lumen (Figure 2.1). Angiogenesis is completed by ECM reestablishment and pericyte recruitment to form a stable, mature vasculature (Figure 2.1).

During tumor angiogenesis, ECs proliferate much faster than in normal vessels [37]. Combined with the absence of a stabilizing maturation phase, this results in a highly irregular, maze-like, inefficient and leaky vasculature with heterogeneous flow profiles and prolonged transit times [38]. 
The third mechanism is arteriogenesis, which is the growth and outward remodeling of pre-existing collateral vessels [39-41]. It is primarily involved in maintaining blood flow in limbs and organs after occlusion of a major conduit artery. Opposed to angiogenesis, arteriogenesis is not activated by tissue hypoxia [40]. In femoral artery occlusion for instance, there is a large distance between the area of collateralization (the upper leg) and the potentially hypoxic tissue (the lower leg and foot, Figure 2.2). Instead, arteriogenesis seems to be mainly flow regulated and is initiated by changes in physical forces [41]. Upon arterial occlusion, blood flow is redirected through the collaterals and shear stress is increased on the vessel wall (24). As a result, collateral arteries enlarge and animal studies showed that they can grow over 20-fold of their original size [41]. Arteriogenesis is completed by a maturation and remodeling step, also called 'pruning', during which many small vessels regress in favor of several larger conduit vessels, which conduct the majority of the total blood flow [39]. The collateral arteries have not only increased in diameter, but have also gained almost a factor two in length between the fixed begin and end points, which makes them tortuous and more resistant to blood flow. Consequently, collateral vessels can only partly (maximal 50\%) replace the occluded artery [39]. During rest, this decreased conductance is usually sufficient to maintain normal organ/limb functionality, but during exercise ischemia might manifest.

Table 2.1 summarizes the morphological and functional characteristics of angiogenic and arteriogenic blood vessels. Imaging of these two mechanisms will be the main focus of this review.

\subsection{Morphological imaging}

Vascular morphology may be studied on a macroscopic and a microscopic scale using contrast-enhanced magnetic resonance angiography and vessel size imaging, respectively. Below, we will discuss how these methods enhance the contrast between vessels and the surrounding tissue, as well as their abilities to probe the structural characteristics of the developing vasculature.

\section{Contrast-enhanced MR angiography}

During contrast-enhanced magnetic resonance angiography (CE-MRA), the blood signal is selectively enhanced by intravenous injection of gadolinium chelates, which shorten the $T_{1}$ relaxation time of blood [42]. On fast 3D gradient echo images recorded with short repetition time (TR), the signal of background tissue is saturated (i.e. suppressed by the rapidly repeating pulses), resulting in a large contrast between blood and tissue. The main advantages of CE-MRA over non-enhanced time-of-flight and phase-contrast MRA, are elimination of blood saturation artifacts, considerably reduced scan time [43], and most importantly, the ability to visualize small vessels ( $<1 \mathrm{~mm}$ caliber). In addition, fewer and thinner slices to cover more vascular anatomy can be recorded in less time by acquisition of 3D images in the plane of the 
arteries of interest. Moreover, image contrast can be improved by proper timing of the arterial phase contrast bolus [44]. As an example, a peripheral CE-MRA exam of a patient with bilateral peripheral arterial occlusive disease (PAOD) is shown in Figure 2.2C.

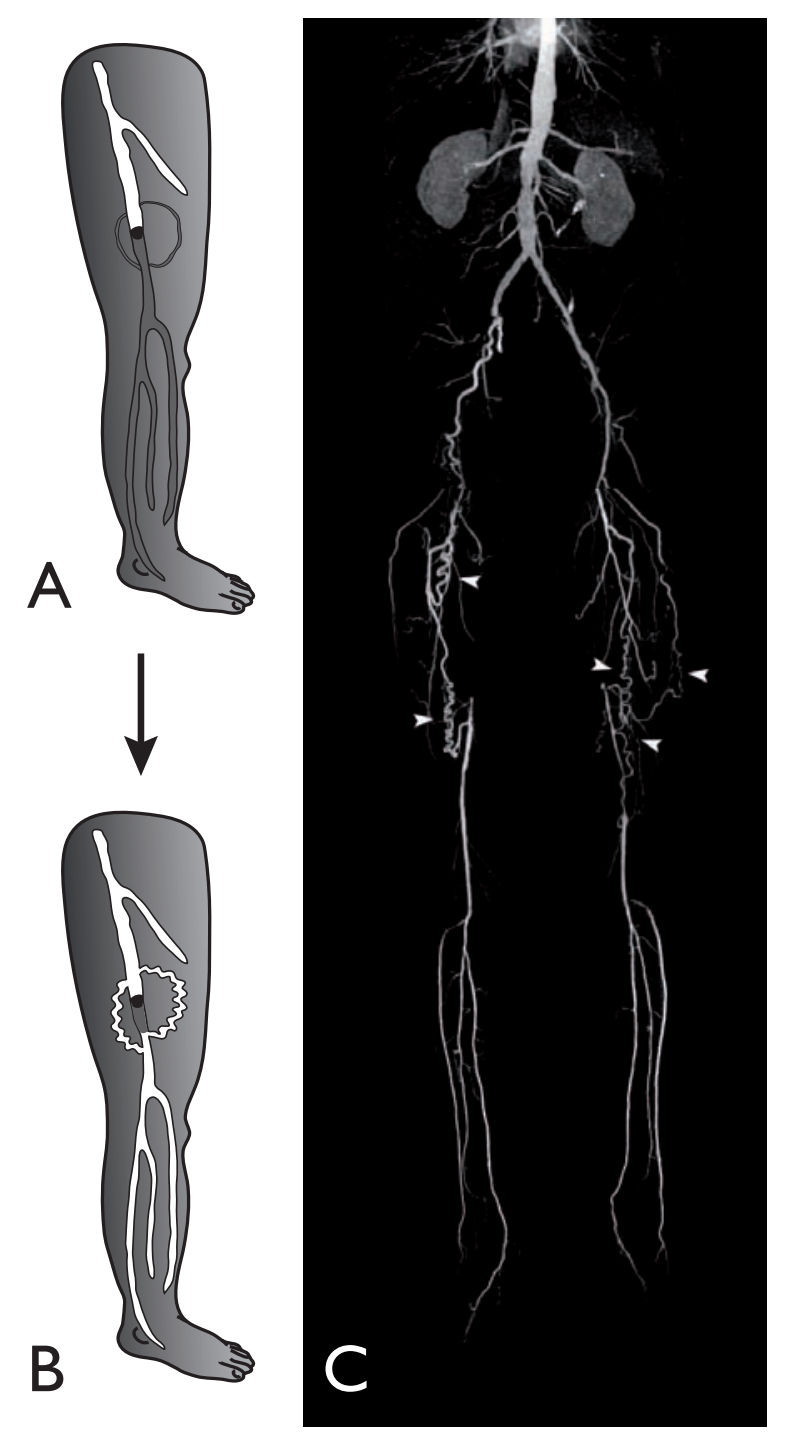

Figure 2.2: Arteriogenesis in the leg of a patient with peripheral arterial occlusive disease (PAOD). A) Occlusion of a conduit artery (black clot) in the thigh results in diminished blood supply to the proximal arteries and ischemia of the lower leg and foot. B) Tortuous collateral arteries have developed and restore perfusion to some extent. C) Multi-station peripheral CE-MRA obtained using Gd-DTPA of a PAOD patient clearly showing a fully developed collateral circulation in both legs (arrowheads). Voxel size $0.9 \times 0.9 \times 1.20 \mathrm{~mm}^{3}$, total scan time 1.5 minutes. Panel C courtesy of Bas Versluis MD, Tim Leiner MD PhD and Walter Backes PhD. 
Table 2.1: Morphological and functional properties of angiogenic and arteriogenic vasculature. Suitable contrast-enhanced MRI techniques to investigate each property are provided.

\begin{tabular}{|c|c|c|c|}
\hline Category & Angiogenesis & Arteriogenesis & MRI techniques ${ }^{a}$ \\
\hline \multicolumn{4}{|l|}{ Morphology } \\
\hline & Spatially heterogeneous & Tortuous & CE-MRA \\
\hline & Irregular diameter & Corkscrew-like & VSI \\
\hline & Arteries and veins not differentiable & Dilated & \\
\hline \multirow{2}{*}{\multicolumn{4}{|c|}{ Function }} \\
\hline & & & \\
\hline \multirow[t]{2}{*}{ Blood perfusion } & Increased & Increased & DCE-MRI \\
\hline & & & Myocardial perfusion imaging \\
\hline \multirow[t]{3}{*}{ Blood flow } & Increased & Increased & DCE-MRI with small CA \\
\hline & Alternating direction & Unidirectional & Myocardial perfusion imaging \\
\hline & & Non-laminar & \\
\hline Blood volume & Increased & Increased & $\begin{array}{l}\text { DCE-MRI with macromolecular CA } \\
\text { VSI }\end{array}$ \\
\hline Permeability & Increased & Increased in early stage only & DCE-MRI \\
\hline
\end{tabular}

${ }^{a}$ CE-MRA = Contrast-enhanced magnetic resonance angiography, VSI = Vessel size imaging, DCE-MRI = Dynamic contrast-enhanced MRI, $C A=$ Contrast agent. Source: references $[38,39,41]$. 
Accurate timing of data acquisition to the contrast agent arrival into the vessels of interest is of crucial importance for optimal arterial contrast. Mistiming is probably the most common error in CE-MRA. Since $T_{1}$ decreases strongly with increasing gadolinium concentration, the highest arterial signal is achieved when images are recorded during the first pass of the contrast agent through the tissue. When image acquisition is too long or delayed, interpretation is complicated by enhancement of venous blood. Mistiming can, for instance, be avoided by employing a timing sequence prior to the examination using a test bolus, or by real-time identification of bolus arrival using MR fluoroscopy [43-45].

CE-MRA can easily depict relatively large mature vessels ( $\geq 1 \mathrm{~mm}$ caliber). Small vessels $(<1 \mathrm{~mm})$ however, demand high spatial resolution, and consequently long acquisition times, which can result in venous contamination. This may be reduced by using advanced gradient systems, dedicated coils suited for parallel imaging, and/or magnets with higher magnetic field strengths. Furthermore, higher resolution can be obtained with partial Fourier and other undersampling techniques, such as k-t BLAST or TRICKS, which tend to work well with sparse data-sets like arterial phase CE-MRA [44].

Using a high spatial resolution $\left(0.6 \times 0.6 \times 0.7 \mathrm{~mm}^{3}\right)$ acquisition, collateral arteries in rabbit hind limbs with a diameter larger than $0.3 \mathrm{~mm}$, could be accurately visualized with CE-MRA on a clinical $1.5 \mathrm{~T}$ scanner equipped with a 5-element phased array coil, and using a 9.5 ms TR [46] (Figure 2.3). Collateral visualization correlated well with X-ray angiography (XRA), although the number of detected collaterals was significantly lower with MRA than with XRA due to the higher spatial resolution of XRA $(\approx 0.25 \mathrm{~mm})$.

In a clinical study by Nijenhuis et al., CE-MRA was applied successfully to localize collateral vessels that contribute to the spinal cord blood supply in thoracoabdominal aorta aneurysm patients [47]. Pre-operative identification of the blood supplying trajectories to the spinal cord is considered relevant to avoid spinal cord ischemia and injury during intra-operative aortic cross-clamping. Using voxel sizes of $0.8 \times 0.8 \times 1.2 \mathrm{~mm}^{3}$, collateral vessels that were critical, and not just accessory, for spinal cord function could be identified (Figure 2.4) [48]. A second clinical example of CE-MRA is the visualization of collateral vessels in patients with a cavernous transformation of the portal vein [49]. Here, collateral vessels are largely pathological and visualization is important for diagnosis and subsequent surgical treatment planning.

Taken together, these examples demonstrate that CE-MRA is suitable for visualizing small, critically functional, or pathological vessels in clinical and preclinical situations. In rodents however, CE-MRA remains challenging because of the animals' appreciably high heart rate and short circulation time. Mice typically have a heart rate of 500-700 beats per minute and a circulation time of 5-10 seconds [50], which precludes first-pass CE-MRA. 


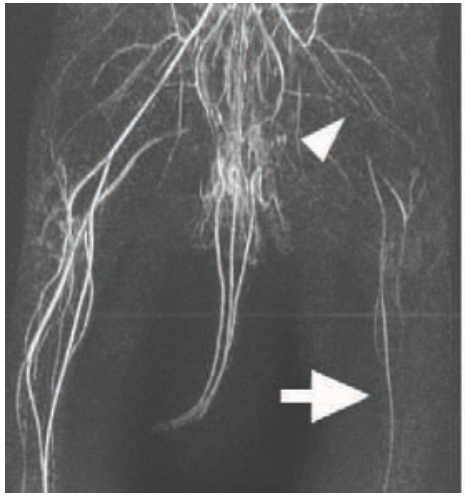

Day $0+$

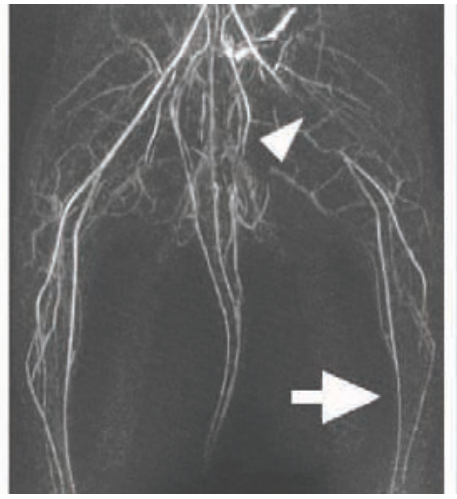

Day 7

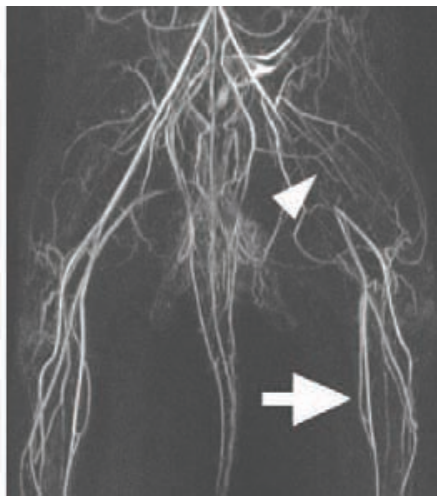

Day 21

Figure 2.3: Maximum intensity projections of CE-MRA images obtained using Gd-DTPA of rabbit hind limbs after femoral artery ligation (day $0+$ : immediately after surgery). Collateral vessels can be clearly discerned at day 7 and even more at day 21 . Restored blood flow in the ligated limb can be observed (arrows) at day 21. Arrow head: ligation site. (Reprinted, with permission, from reference [46])

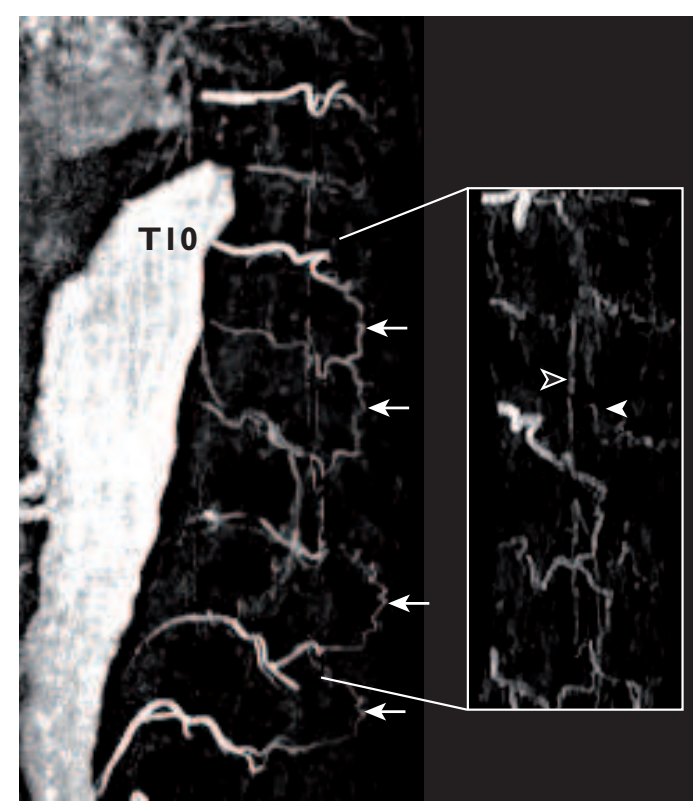

Figure 2.4: Targeted maximum intensity projections of a CE-MRA, recorded using Gd-DTPA, of the aneurysm and segmental arteries of a thoracoabdominal aortic aneurysm patient (Crawford type II). Most segmental arteries are occluded and are indirectly supplied via an extensive collateral network (arrows) originating from segmental artery T10 and lumbar circulation. The inset shows the Adamkiewicz artery (white arrowhead) and the anterior spinal artery (black arrowhead), which are responsible for the spinal cord blood supply. The Adamkiewicz artery is supplied via a collateral originating from the T10 segmental artery. Figure courtesy of Robbert Nijenhuis MD PhD and Walter Backes PhD. 


\section{Blood pool agents}

An inherent problem of CE-MRA using many of the currently available gadolinium chelates, is the rapid leakage of these small contrast agents (e.g. Gd-DTPA, $576 \mathrm{Da}$ ) from the vasculature into the interstitium. This occurs in both healthy and pathological vessels and results in extravascular signal enhancement and decreased vessel to background contrast. Contrast extravasation can be reduced by the use of macromolecular contrast agents [51-53]. These so-called blood pool agents have molecular weights of several $\mathrm{kDa}$ and reside largely intravascular in normal tissues. In hyperpermeable tumor vessels however, these agents do leak into the extracellular space. Examples of macromolecular contrast agents are dendrimers (highly branched polymers), albumin-Gd-DTPA and the albumin binding agent Vasovist ${ }^{\text {TM }}$ (Bayer Schering Pharma AG, Berlin, Germany). Most of these agents have not been clinically approved yet and have mainly been used in animal studies.

Steady-state CE-MRA images with a spatial resolution of approximately $200 \mu \mathrm{m}$ were obtained in mouse tumors by Fink et al. on a clinical 1.5 T MRI scanner using the dendritic contrast agent Gadomer ${ }^{\circledR}(17 \mathrm{kDa}, 24$ gadolinium chelates, Bayer Schering Pharma AG, Berlin, Germany) [54]. Blood vessels both outside and inside the tumor could be depicted. Subjective strong and weak contrast enhancement in the tumor correlated with highly vascularized and necrotic regions, respectively (Figure 2.5). Due to the 8 minute acquisition time, it is likely that both arteries and veins were visualized.

\section{Vessel size imaging}

Vessel size imaging ( $\mathrm{VSI}$ ) probes the distribution of submillimeter caliber microvessels using the ratio of changes in the transverse relaxation rates $R_{2}^{*}$ and $R_{2}\left(\Delta R_{2}^{*} / \Delta R_{2}\right)$, induced by a superparamagnetic contrast agent. In the future, VSI may for instance be applied to infer patient diagnosis and prognosis, since tumor vascularity was shown to be related to tumor aggressiveness (e.g. [55]). Additionally, it may be used to monitor the response upon anti-angiogenic treatment.

Using mathematical models of vessel distribution within tumor voxels and Monte Carlo simulations, it was shown by several authors that the ratio of $\Delta R_{2}^{*}$ and $\Delta R_{2}$ is a direct measure of the average vessel radius $R_{v}$ (also called vessel size index):

$$
R_{V}=0.424 \cdot\left(\frac{D}{\gamma \Delta \chi B_{0}}\right)^{1 / 2}\left(\frac{\Delta R_{2}^{*}}{\Delta R_{2}}\right)^{3 / 2}
$$

where $D$ is the apparent diffusion coefficient of water, $\gamma$ the gyromagnetic ratio, $\Delta \chi$ the increase in blood susceptibility due to the contrast agent, and $B_{0}$ the magnetic field strength [56-59]. Furthermore, the fractional blood volume $\xi$ can be extracted using: 


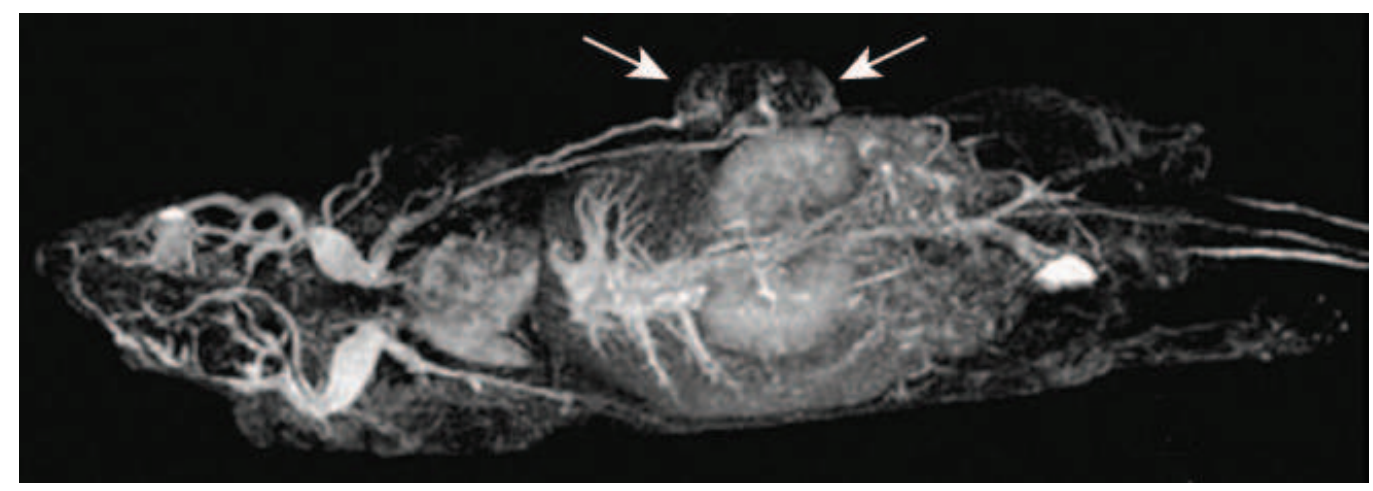

Figure 2.5: Maximum intensity projection of a high resolution $\left(166 \times 206 \times 320 \mu \mathrm{m}^{3}\right)$ whole body CE-MRA of a nude mouse carrying a subcutaneous tumor on the flank (arrows), recorded using a medium-sized contrast agent (Gadomer). Blood vessels can be observed in the tumor periphery. (Reprinted, with permission, from reference [54]).

$$
\xi=\frac{3}{4 \pi} \frac{\Delta R_{2}^{*}}{\gamma \Delta \chi B_{0}}
$$

For more detailed information on the theory of VSI, the reader is referred to [56-59].

A difficulty of VSI is its dependency on $\Delta \chi$ and, consequently, contrast agent concentration. Jensen and Chandra deduced that the concentration of superparamagnetic iron oxide particles should be well over $0.1 \cdot B_{0} \mathrm{mM} \mathrm{Fe} / T$ to obtain reliable $R_{V}$ values and defined a concentration independent measure of vessel size to circumvent this problem [57]. For low molecular weight contrast agents, VSI is hampered by contrast agent leakage and macromolecular agents are consequently preferred. The intrinsic venous blood paramagnetism may result in systematic overestimation of vessel size, although this is less problematic for contrast agents with high susceptibility. Finally, accurate VSI measurements require knowledge of the local diffusion coefficient, since this may increase by a factor of two in tumors compared to normal tissue $[59,60]$. Only average diffusion values obtained from literature were used in the previously cited studies.

VSI in rat brain tumors showed that tumors had a significantly higher $R_{V}$ than healthy brain tissue [59]. A good correlation with histology was found, especially for small $R_{v}$. However, histology measures both perfused and non-perfused vessels, whereas VSI probes only the perfused vessels. VSI is furthermore suitable for basic research regarding the mechanisms of vessel growth. Using a mouse tumor model, Robinson et al. showed that VSI allows assessment of the role of platelet-derived growth factor on vessel maturation and pericyte coverage [61]. 
A clinical application of VSI was described by Kiselev et al., who found an increased $R_{V}$ in malignant versus benign human brain tumors when using Gd-DTPA as contrast agent, suggesting that VSI is sensitive to brain tumor type. This might be beneficial for patient diagnosis, prognosis and treatment planning. Although vessel size appeared to be overestimated by a factor of two, this might be reduced for intravascular contrast agents with greater susceptibility effects. Alternatively, the overestimation may be related to the underlying tissue model, which assumed a uniform vessel distribution, while tumor vessels are generally heterogeneous. Furthermore, only a limited number of patients was included.

An example of VSI to monitor tumor growth outside the brain was recently provided by Wade and Kozlowski [62]. Here, VSI included measurement of tissue diffusion and was applied to a mouse model of prostate cancer. A significantly lower $R_{V}$ was found in regressing tumors after castration compared with growing tumors.

\subsection{Functional imaging}

Vascular function is key to organ viability and function. Abnormalities in vessel function, for instance reduced flow or increased vessel wall permeability, often occur at the onset of the disease, before the patient becomes symptomatic. Consequently, detection of vessel function is important for early patient diagnosis, prognosis and for the evaluation of treatments aimed at improving vessel function.

A well-studied contrast-enhanced MRI method to measure vessel function is dynamic contrast-enhanced MRI, which will be the main topic of this section. A related technique to measure myocardial perfusion will be discussed shortly thereafter.

\section{Dynamic contrast-enhanced MRI}

With dynamic contrast-enhanced MRI (DCE-MRI), physiological information on blood flow, vessel wall permeability and/or surface area of microvessels can be obtained by $T_{1}$-weighted imaging. It exploits the leakage of contrast agents out of the vasculature and is therefore highly suitable to investigate tumor angiogenesis due to the increased vascularity and permeability, and abnormal, i.e. spatially and/or temporarily heterogeneous, perfusion that is present in these lesions [63]. Consequently, many of the clinical and preclinical DCE-MRI studies focused on the tumor microvasculature and applied DCE-MRI in oncological studies evaluating the efficacy of anti-angiogenic medication $[22,64,65]$.

The kinetic behavior of contrast agents is mainly determined by three factors: blood perfusion, active transport, and passive diffusion of contrast agent through the vessel wall into the extravascular-extracellular space (EES) [22]. The rate of transendothelial transport of a contrast agent is described by the volume transfer 

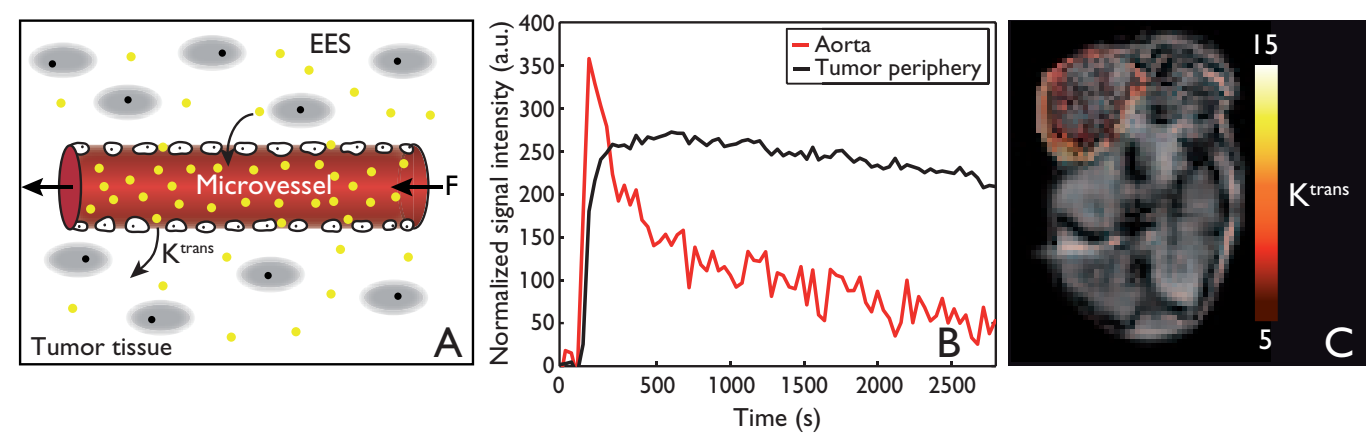

Figure 2.6: Schematic representation of the parameters that can be extracted from DCEMRI data. See text and Appendix A for parameter explanation. Red: tumor microvessel, Grey ovals: tumor cells, Yellow dots: contrast agent molecules. B) DCE-MRI signal curves in the aorta and the tumor periphery of a nude mouse carrying a subcutaneous human colon carcinoma on the flank (top left in panel C). Gd-DTPA was injected at time 0 and 3D gradient echo images were obtained at a temporal resolution of 40 seconds. C) Corresponding $K^{\text {trans }}$ map (in $\mathrm{mL} \cdot 100 \mathrm{~cm}^{-3} \cdot \mathrm{min}^{-1}$ ). Tumor heterogeneity is clearly visible with higher $K^{\text {trans }}$ at the tumor rim.

coefficient $K^{\text {trans }}$ (Figure 2.6), which is defined as the product of the vascular flow $F$ and the extraction fraction $E$, with $P S$ the permeability surface area product:

$$
E=1-e^{-P S / F}
$$

Alternatively, $T_{2}^{*}$-weighted DCE-MRI (i.e. susceptibility enhanced MRI) may be used to assess tumor regional blood volume, blood flow, and mean transit time. However, as previous research has focused mainly on $T_{1}$-weighted DCE-MRI, we will not further discuss susceptibility enhanced MRI and refer the reader to [66] and references therein.

\section{Data acquisition}

To monitor contrast agent kinetics, usually a large number $(>50)$ of gradient echo images are serially recorded at a high temporal resolution ( $<40$ seconds). Images can be obtained in $2 \mathrm{D}$ or $3 \mathrm{D}$ mode. $2 \mathrm{D}$ acquisition allows a higher temporal resolution but is more sensitive to inflow artifacts, whereas 3D sequences are more suitable for imaging large field of views [67]. The contrast agent is usually injected after multiple measurements of the baseline signal. It is assumed that all resulting signal intensity changes reflect changes in contrast agent concentration. An example of the temporal signal in the aorta and tumor of a mouse is shown in Figure 2.6. A sharp rise in signal intensity is observed in the aorta immediately after contrast agent injection, which is followed by a bi-exponential decay due to rapid distribution and plasma clearance. In the tumor, a less steep increase and a more gradual clearance are observed. High 
temporal resolution is a prerequisite for accurate registration and quantification of these time courses. This frequently necessitates a reduction in spatial resolution, anatomical coverage or even both. Mathematical details concerning the pharmacokinetic model which can be applied to extract $K^{\text {trans }}$, the relative plasma volume $\left(v_{p}\right)$, and the relative EES volume $\left(v_{\mathrm{e}}\right)$ from the temporal signal curves are provided in Appendix A.

\section{Vascular input function}

Accurate measurement of the blood signal, also called the vascular input function (VIF), is important for reliable estimation of the pharmacokinetic parameters. However, VIF measurements are complicated by several factors, including a strong dependence on measurement location, saturation inflow effects, high contrast agent concentration ( $T_{2}^{*}$-effects, vide infra) and partial volume effects. In general, two main methods can be discerned to obtain a VIF: (i) individual VIF measurement and (ii) the use of a previously determined group average VIF. The former has the advantage that it corrects for individual variations in injection speed, vascular resistance, clearance rate, cardiac output, etc. An important disadvantage is the required high temporal and spatial resolution to ascertain accurate data sampling, and to avoid inflow and partial volume effects, respectively. This is usually achieved at the expense of SNR. Advantages of an average VIF are that it has a considerably better SNR and can be applied in situations where measurement of the individual VIF failed [68]. However, errors of up to $30 \%$ in estimated pharmacokinetic parameters have been reported when using an average VIF [69].

A reference region input function has been suggested as an alternative to the VIF. It is less prone to errors caused by inflow and partial volume effects and can be obtained in a more standardized way at a lower temporal resolution [70-72]. Consequently, a higher spatial resolution might be obtained and tissue heterogeneity can be investigated more accurately [70]. The reference region model is, however, still under development and has to our knowledge only been applied in preclinical models. Furthermore, either $K^{\text {trans }}$ and $v_{\mathrm{e}}$ or only $v_{\mathrm{e}}$ of the reference region are fixed during calculation, which potentially introduces bias in the estimated pharmacokinetic parameters.

At high intravascular concentrations, $T_{2}^{*}$-effects have a considerable influence on estimated contrast agent concentrations. Figure 2.7 shows the observed signal amplification as function of gadolinium concentration. Considering the contrast agent's $T_{1}$-effect only, a linear relationship is observed at the low concentrations typically found in tissues (also known as the fast exchange limit), whereas a significant deviation from linearity arises at higher concentrations. When the echo time and $T_{2}^{*}$ are in the same order of magnitude, $T_{2}^{*}$ shortening further decreases the signal amplification. Consequently, the high gadolinium concentrations of the VIF are largely underestimated and $K^{\text {trans }}$ will be overestimated accordingly. 


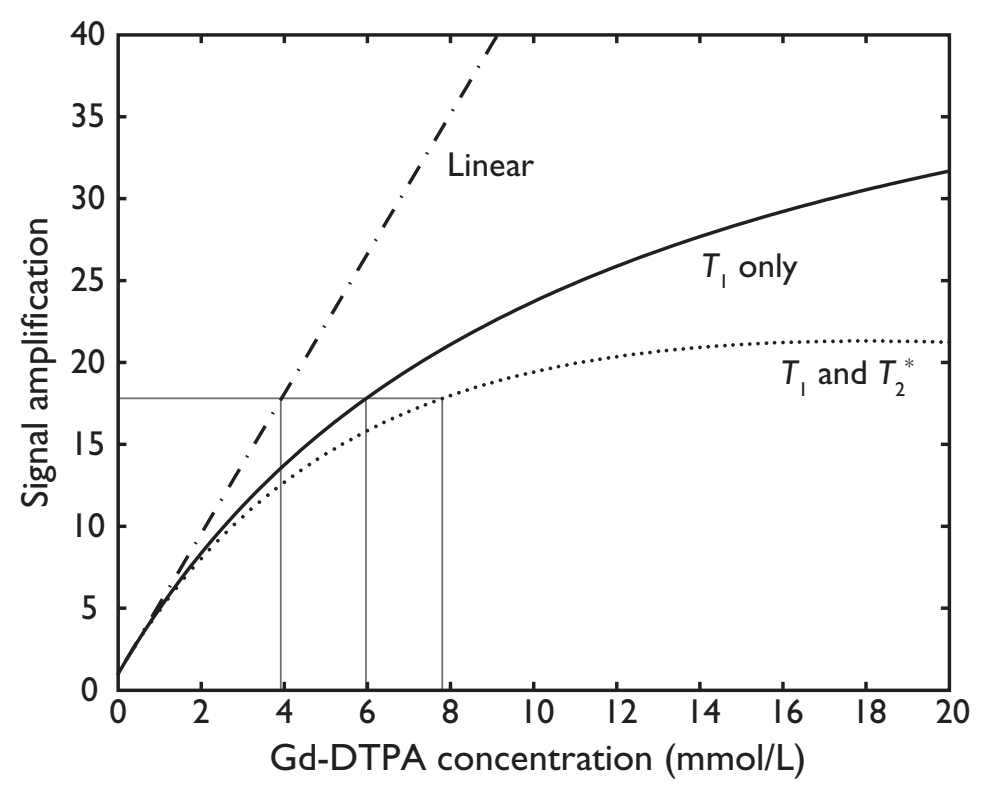

Figure 2.7: Signal amplification versus Gd-DTPA concentration for DCE-MRI measurements according to equation A.3 of Appendix A. For low concentrations, a linear behavior is observed (fast exchange limit, dot-dashed line). When taking $T_{1}$-effects into account, a clear deviation from linearity is observed (solid line). If $T_{2}^{*}$-effects are also considered (dotted line), an even stronger deviation is found, especially for high concentrations. At a given signal amplification, a linear or $T_{1}$ corrected model will give significant underestimations of Gd-DTPA concentration (solid vertical and horizontal lines).

De Bazelaire et al. found that at field strengths of $3 \mathrm{~T}$ and higher, $T_{2}^{*}$-effects resulted in a signal reduction of more than 20\% [73]. Using a combined $T_{2}^{*}$ and $T_{1}$ measurement, they were able to reduce the underestimation of gadolinium concentration and the $K^{\text {trans }}$ overestimation by 59 and $58 \%$, respectively. However, tissue and field strength inhomogeneities may lead to uncertainties in these corrections. Further improvements in the accuracy of VIF extraction were described by Cheng, who implemented specific algorithms to correct for $B_{1}$ field imperfections, in-flow, and partial volume effects [74].

Finally, the translation of signal intensity changes to local concentrations of contrast agent is complicated by variations in contrast agent $T_{1}$ relaxivity. For instance, it was found that Gd-DTPA relaxivity increases with increasing macromolecular concentration, with a 30-70\% higher value in the EES than in saline [16].

\section{Model validation}

Currently, no suitable validation is available for $K^{\text {trans }}, v_{e}$, and $v_{p}$ measurements. Especially for $K^{\text {trans }}$, validation is complicated due to its dependency on vessel wall 
permeability, flow, and contrast agent size. For a low molecular weight contrast agent like Gd-DTPA, $K^{\text {trans }}$ primarily reflects permeability under low flow conditions, while under high flow conditions it largely reflects flow. For macromolecular contrast agents like dendrimers, $K^{\text {trans }}$ mainly reflects the permeability surface area product $[75,76]$.

An alternative method to measure blood flow is laser Doppler imaging [77]. This method however, cannot validate DCE-MRI as the limited penetration depth of light implies that it reflects mostly skin blood flow. Fluorescence microscopy [78], microspheres for regional blood flow [79], and Evan's blue staining for permeability assessment, may be appropriate validation tools for DCE-MRI. However, their invasive nature hampers widespread testing under non-perturbed experimental conditions. Nevertheless, Lüdemann et al. published that similar results were obtained for myocardial perfusion in pigs measured with either DCE-MRI or colored microspheres [80]. Microsphere perfusion measurements in rat tumors were also found to correlate well with DCE-MRI measurements obtained using deuterated water as diffusible tracer [69].

In several tumor angiogenesis studies, DCE-MRI was compared to the histologically determined microvessel density (MVD) [81-84]. Although a positive correlation was found, MVD is not considered an accurate validation method, because it measures both functional and non-functional vessels, whereas DCE-MRI probes only functional vessels.

\section{Model accuracy}

Using numerical simulations, Buckley et al. demonstrated that three commonly accepted pharmacokinetic models, i.e. the Kety model, the Kety model extended with a vascular term, and the model of St. Lawrence and Lee, all result in a systematic overestimation of $K^{\text {trans }}$ and a potential underestimation of $v_{p}$ for small molecular contrast agents [85]. Lopata et al. found that the most accurate assessment of $K^{\text {trans }}$ was obtained at high temporal resolutions and rapid contrast agent injection [86]. The critical parameter determining the sample frequency was the ratio $K^{\text {trans }} / V_{\mathrm{e}}$. It was furthermore reasoned that more accurate parameter estimations can be achieved when using medium or large-sized contrast agents with slower pharmacokinetic properties. This will give a reduction in $K^{\text {trans }}$ and consequently lower sampling rates can be used, which will result in a higher SNR and/or spatial resolution in the obtained images [86]. This was supported by Kiessling et al., who argued that medium-size dendrimers (20-30 kDa) are preferred for clinical DCE-MRI due to their reduced leakage out of the vasculature and favorable body clearance and renal accumulation rates [67]. However, these contrast agents are currently not approved for clinical use.

Tumors often contain areas of lymphatic, fibrotic, and necrotic tissue, which can form additional compartments where the contrast agent can leak into with different time constants. Consequently, more compartments may be required to accurately 


\section{CHAPTER 2. MRI OF VESSEL GROWTH AND FUNCTION}

describe the pharmacokinetics of the tumor microenvironment [87]. Also, the presence of an intracellular compartment was proposed. Although the contrast agent concentration in this compartment is zero, intracellular water contributes to the observed signal via transcytolemnal water exchange [88]. Incorporation of the intracellular compartment into the pharmacokinetic model resulted in improved fittings of the signal time courses and larger $K^{\text {trans }}$ values compared with the traditional two-compartment model. The use of multiple compartments may furthermore be important for macromolecular contrast agents that can, for instance, bind to plasma proteins, be captured in macrophages or even be metabolized by the tumor [67].

Using additional compartments also introduces uncertainties, as multiple, equally good mathematical solutions may exist. In a two-compartment model there is already a strong interdependency between $v_{e}$ and $v_{p}$, and a large variance in $K^{\text {trans }}$ when using sub-optimal experimental settings [86]. Furthermore, the absolute benefits of a multiple compartment model still have to be demonstrated. Currently, the only objective parameter to compare different models is the quality of the fit - that is, how well does the model describe the experimental data? However, it remains impossible to determine which model truly reflects the local flow, permeability, and/or vascularity, since absolute validation of the pharmacokinetic parameters is currently unavailable, as indicated previously.

Regarding the reproducibility of DCE-MRI, a study in normal human pelvic tissues showed a large between-patient variance of kinetic parameters compared to the mean values [89]. It was found that a logarithmic change in $K^{\text {trans }}$ of $0.89 \mathrm{~min}^{-1}$ for muscle tissue is required to detect significant individual changes. Compared to the global mean $K^{\text {trans }}\left(\approx 0.14 \mathrm{~min}^{-1}\right)$, this is an extremely large value, which suggests that the reproducibility may be insufficient to be clinically applicable, unless treatment effects are large. A considerably better reproducibility was reported by Galbraith et al., who found that a change of $14-17 \%$ in $K^{\text {trans }}$ would be sufficient to be statistically significant in a cohort of 16 patients [90]. These results were supported by Roberts et al. [91] and Lankester et al. [66]. Considering the differences in reported reproducibility, studies aimed at evaluating treatment response with DCEMRI should incorporate reproducibility measurements to assess variability in both normal and pathological tissues [89]. Alternatively, due to the established temporal heterogeneity in tumor perfusion [63], it might also be justifiable to demand only a sufficient reproducibility on whether $K^{\text {trans }}$ is significantly increased or not, i.e. above a certain normative threshold value, and not to require an exact replication of $K^{\text {trans }}$.

\section{Applications}

DCE-MRI is becoming a valuable tool for cancer diagnosis. It allows accurate depiction of tumor heterogeneity, with higher $K^{\text {trans }}$ in the rim of the tumor and lower $K^{\text {trans }}$ in the core (Figure 2.6), which corresponds with the higher rate of vessel growth in the tumor rim. The regional variation in $K^{\text {trans }}$ correlated well with MVD 
measurements [81]. Furthermore, using a range from benign to highly malignant mammary tumors in female rats and using a macromolecular contrast agent, Daldrup et al. found that $K^{\text {trans }}$ and $v_{p}$ correlated strongly with histopathologic tumor grade. No correlations were found when using Gd-DTPA [92]. In contrast, in a recent clinical study by Radjenovic et al., DCE-MRI using Gd-DTPA did result in significantly increased $K^{\text {trans }}$ and the reflux rate $K\left(=K^{\text {trans }} / V_{e}\right)$ in high-grade invasive breast tumors (grade 3) compared with low grade tumors (grade 1 and 2) [93]. These experimental findings indicate that DCE-MRI may allow differentiation between benign and malignant tumors in humans, which could be important for patient prognosis and treatment planning.

Furthermore, DCE-MRI may be applied to assess the effects of traditional chemoradiative therapy and emerging anti-angiogenic therapies. For instance, a significant decrease in $K^{\text {trans }}$ was found upon treatment of tumor bearing mice with the angiogenesis inhibitors Anginex and TNP-470 [81]. In addition, the applicability of DCE-MRI to monitor the effects of anti-vascular therapy was also demonstrated in several clinical trials [94].

Other oncological applications of DCE-MRI include screening women with high genetic risk of breast cancer, evaluation of the contralateral breast in women diagnosed with unilateral breast cancer, and the assessment of tumor stage and therapy response in other common cancer types such as rectal, prostate and cervical cancers $[22,64,95,96]$.

A cardiovascular example of DCE-MRI was described by Kerwin et al., who studied carotid atherosclerotic plaque neovascularization in patients scheduled for carotid endarterectomy $[97,98]$. A significant correlation was found between $K^{\text {trans }}$, determined using Gd-DTPA, and the histologically determined macrophage, neovasculature, and loose matrix content, suggesting that $K^{\text {trans }}$ is a quantitative, non-invasive marker of plaque inflammation and the extent of neovascularization [98]. Since increased angiogenesis and inflammation are likely related to atherosclerotic lesion progression [99], DCE-MRI may become a suitable tool to non-invasively study the relationship between neovascularization and plaque stability in vivo.

Recently, DCE-MRI was also employed to characterize the vasa vasorum in the adventitia of atherosclerotic arteries [100]. A significantly higher adventitial $K^{\text {trans }}$ was found in patients with severe lesions compared to patients with only moderate disease. Histological validation showed that $K^{\text {trans }}$ correlated well with the amount of adventitial neovasculature, indicating that the extent of the vasa vasorum may be inferred from DCE-MRI measurements.

\section{Myocardial perfusion imaging}

Due to cardiac and respiratory movement, a dedicated method is required to measure myocardial perfusion and to detect ischemia caused by obstruction of one or multiple 
coronary arteries. Myocardial perfusion imaging is based on the dynamic recording of the first-pass of a gadolinium contrast agent. During bolus passage, the contrast agent enters the microvasculature and starts to distribute over the interstitial space, thereby enhancing the myocardial signal. The rate of signal enhancement is directly related to the perfusion state of the tissue: normally perfused tissue will display a fast increase whereas hypoperfused myocardium will show a delayed increase. Differences between normal and ischemic myocardium can be augmented using pharmacological stress agents such as adenosine or dipyridamole. These drugs induce vasodilation and consequently increase blood flow in healthy myocardium, but not in ischemic areas. The ratio of rest and stress perfusion is defined as the perfusion reserve and is inversely related to the severity of coronary artery stenosis [101].

Images are generally acquired at a temporal resolution of at least every other heartbeat at a spatial resolution of 2-3 mm using fast read-outs such as gradient echo, echo planar imaging or steady state free precession [102,103]. Furthermore, due to the short duration of the first contrast agent passage, data acquisition is usually performed during breath-hold, which results in diminished respiratory motion. The effects of cardiac motion can be reduced using ECG-triggering. Technical limitations currently prevent the application of myocardial perfusion imaging to rodents because of their rapid cardiac and respiratory movement.

Myocardial perfusion imaging data can be analyzed qualitatively by visually inspecting the signal intensity - time curves; by using semi-quantitative measures as upslope, peak signal intensity, or myocardial transit time; or by means of absolute quantification of tissue perfusion [102]. As described previously, quantification requires knowledge of an arterial input function to correct for individual differences in contrast agent delivery from the left ventricle to the myocardium. Myocardial blood flow can subsequently be calculated using model-independent deconvolution techniques [104]. In pigs, the resulting blood flow correlated well with microsphere measurements.

After myocardial infarction, tissue damage may be limited or even prevented if a functional collateral network is present [105]. Non-invasive collateral detection may therefore be valuable for periodical patient follow-up, to identify high-risk areas of the myocardium, or to estimate patient prognosis after myocardial infarction. Both preclinical and clinical studies have shown that the time delay of contrast agent arrival in the myocardium allows differentiation between healthy and collateral-dependent myocardium $[106,107]$. Absolute quantification of myocardial blood flow was only able to detect differences between healthy tissue and tissue perfused via a collateral network during adenosine-induced stress [106]. However, further investigation and validation are still needed to assess the true potential of this method. 


\subsection{Molecular imaging}

Molecular imaging is defined as the in vivo visual representation, characterization and quantification of biological processes and molecular pathways at a cellular and molecular level [108]. More specifically, molecular imaging commonly uses targeted contrast agents that home to disease-specific markers (e.g. receptors, enzymes) to generate contrast at the locations of these markers (Figure 2.8). Provided that a suitable target, probe, and detection method are available, the concept can be incorporated in various imaging modalities, such as MRI, CT, PET, SPECT, ultrasound, and optical imaging. A potential advantage of molecular imaging over traditional imaging is disease specificity, while simultaneously providing information on disease localization. Hypothetically, this could lead to a more accurate diagnosis at an earlier stage of the disease process [109]. Advantages of using MR molecular imaging over the other modalities are the excellent spatial resolution and soft tissue contrast, the absence of ionizing radiation, the fact that images can be recorded in any oblique direction, and the possibility to obtain anatomical, functional as well as molecular information within a single imaging session. A major disadvantage of MRI is the inherent low sensitivity.

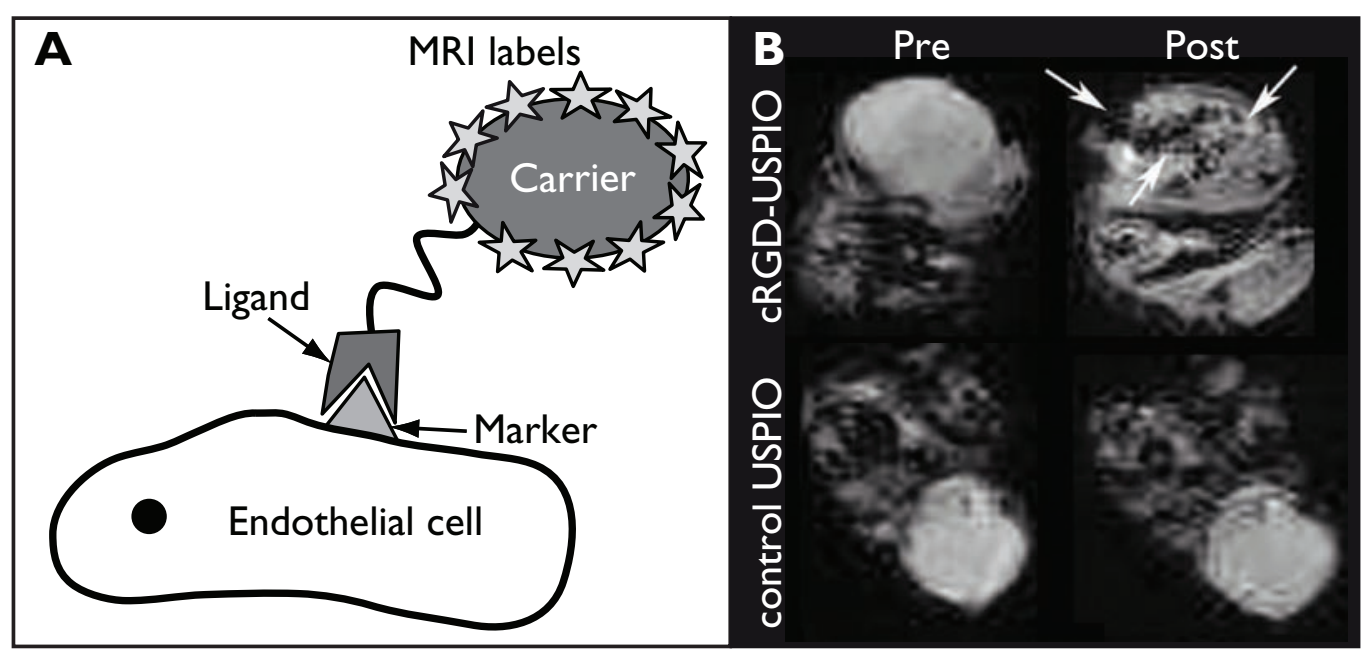

Figure 2.8: A) Schematic representation of the principles of molecular MRI. A carrier particle containing many MRI labels is targeted to a specific endothelial cell marker via high affinity ligands. MRI labels can be for instance gadolinium or iron. For iron oxide particles, additional MRI labels are generally not required since the carrier particle itself is already superparamagnetic. B) In vivo results of molecular MRI of tumor angiogenesis using CRGD-labeled USPIO. Post cRGD-UPSIO injection, a strong signal decrease is observed in the tumor. Only minor signal changes were found upon injection of unlabeled control USPIO. (Figure B reprinted, with permission, from reference [110]). 


\section{CHAPTER 2. MRI OF VESSEL GROWTH AND FUNCTION}

Neovascularization is an attractive phenomenon for molecular imaging because ECs and their molecular targets are more easily accessible for contrast agents than deeply lying tissue cells. However, there are much fewer ECs compared with the number of surrounding tissue cells. In tumors for instance, approximately $10 \%$ of the cells are ECs [111], so volume averaging reduces their signal intensity. Also, the presence of contrast agent in the blood pool may obscure the signal enhancement from receptor-bound contrast agent particles.

Markers that have been used as target for molecular imaging of neovascularization are provided in Table 2.2. Relative to the physiologic state, the level of marker expression is generally increased during disease, although their final concentration is still extremely low. Concentrations are estimated to be in the pico- to nanomolar range, but quantitative measurements are lacking. Consequently, high gadolinium or iron concentrations are required for detection by MRI. This is typically achieved by using large constructs that carry a high payload of gadolinium or iron, and are capable of binding to the target molecule via peptide, antibody or polysaccharide ligands (Figure 2.8). Usually, hundreds to thousands of ligands are coupled to the carrier particle to augment its affinity for the target. It was shown that these multivalent interactions can result in a more than 1000-fold increase in affinity of the cyclic arginine-glycine-asparagine (CRGD) peptide, a commonly applied ligand for molecular MRI of angiogenesis (Table 2.2), for the $\alpha_{v} \beta_{3}$ integrin [112]. Additionally, large carrier particles have a slow tumbling rate, which enhances relaxivity. Serious disadvantages of these large constructs are the poor tissue penetration, rapid plasma clearance, and strong accumulation in the liver, spleen, and kidneys. Furthermore, it is unknown if the large quantities of contrast agent required for molecular MRI interfere with the targeted biological process.

Table 2.2 shows an overview of the angiogenic markers, corresponding ligands, and carrier particles that have been used for molecular MRI. Although none of these particles have been applied clinically, promising results have been obtained in animal research.

Gadolinium-based contrast agents reduce local $T_{1}$, resulting in an increase in signal intensity, and are categorized as positive contrast agents. In contrast, iron oxide-based agents reduce the signal intensity via a local reduction of $T_{2}$ and therefore belong to the negative contrast agents. Theoretically, a higher sensitivity can be achieved with negative iron oxide contrast agents than with positive gadolinium-based agents [110]. Iron oxide particles therefore provide a potential solution for the low sensitivity of molecular MRI. Zhang et al. recently used cRGD-conjugated ultrasmall superparamagnetic iron oxide particles (USPIO) to characterize $\alpha_{v} \beta_{3}$ expression in vitro and in murine tumors [110]. At $1.5 \mathrm{~T}$, a pronounced decrease in signal intensity was observed 6 hours after CRGD-USPIO administration and a clear difference between tumors with high and low $\alpha_{\mathrm{v}} \beta_{3}$ expression patterns was detected by both $T_{2}^{*}$-weighted imaging and $T_{2}$ relaxometry. No significant changes in signal intensity where found for unlabeled USPIO compared with pre-contrast images (Figure 2.8). 
Table 2.2: Targets with corresponding ligands used for molecular MRI of angiogenesis. Physicochemical properties of the carrier particles and the applied experimental model are presented.

\begin{tabular}{|c|c|c|c|c|c|c|}
\hline Target & Ligand & Carrier particle & $\begin{array}{l}\text { Particle } \\
\text { diameter } \\
(\mathrm{nm})\end{array}$ & $\begin{array}{l}\text { Ionic relaxivity } \\
\left(\mathrm{mM}^{-1} \mathrm{~s}^{-1}\right)^{a}\end{array}$ & $\begin{array}{l}\text { Experimental } \\
\text { model }\end{array}$ & Refs \\
\hline \multirow[t]{6}{*}{$\alpha_{\mathrm{v}} \beta_{3}$} & LM609 mAb & $\begin{array}{l}\text { Paramagnetic } \\
\text { polymerized } \\
\text { liposomes }\end{array}$ & $300-350$ & $N D^{b}$ & Rabbit tumor & [19] \\
\hline & $\begin{array}{l}\text { Cylcic RGD } \\
\text { peptide }\end{array}$ & $\begin{array}{l}\text { Paramagnetic and } \\
\text { fluorescent } \\
\text { liposomes }\end{array}$ & $\approx 150$ & $\begin{array}{l}\approx 10 \text { at } 0.6 \mathrm{~T} \\
\approx 5 \text { at } 6.3 \mathrm{~T}\end{array}$ & $\begin{array}{l}\text { Nude mice } \\
\text { bearing human } \\
\text { colon carcinoma }\end{array}$ & $\begin{array}{l}{[113} \\
114]\end{array}$ \\
\hline & $\begin{array}{l}\text { Peptidomimetic } \\
\alpha_{v} \beta_{3} \text { antagonist }\end{array}$ & $\begin{array}{l}\text { Gd-perfluorocarbon } \\
\text { nanoparticle }\end{array}$ & $\approx 270$ & $\begin{array}{l}\approx 20 \mathrm{at} \\
0.47 \mathrm{~T}\end{array}$ & $\begin{array}{l}\text { Nude mice } \\
\text { bearing human } \\
\text { melanoma }\end{array}$ & [115] \\
\hline & $\begin{array}{l}\text { Cyclic RGD } \\
\text { peptide }\end{array}$ & $\begin{array}{l}\text { Gd-perfluorocarbon } \\
\text { nanoparticle }\end{array}$ & $\approx 270$ & 17.7 at $1.5 \mathrm{~T}$ & $\begin{array}{l}\text { Rabbit } \\
\text { atherosclerosis }\end{array}$ & [116] \\
\hline & DM101 mAb & $\begin{array}{l}\text { Gd-perfluorocarbon } \\
\text { nanoparticle }\end{array}$ & $\approx 200$ & 12 at $4.7 \mathrm{~T}$ & $\begin{array}{l}\text { Rabbit corneal } \\
\text { micropocket }\end{array}$ & {$[117]$} \\
\hline & $\begin{array}{l}\text { Cyclic RGD } \\
\text { peptide }\end{array}$ & USPIO & $\approx 10$ & $\begin{array}{l}1 \text { at } 1.5 \mathrm{~T} \\
134 \text { at } 1.5 \mathrm{~T}^{\mathrm{c}}\end{array}$ & $\begin{array}{l}\text { In vitro HUVEC } \\
\text { and in vivo nude } \\
\text { mice bearing } \\
\text { human squamous } \\
\text { cell carcinoma }\end{array}$ & [110] \\
\hline$\alpha_{5} \beta_{1}$ & $\begin{array}{l}\text { Cyclic } \\
\text { ACRGDGWCG } \\
\text { peptide }\end{array}$ & $\begin{array}{l}\text { Gd-perfluorocarbon } \\
\text { nanoparticle }\end{array}$ & $\approx 300$ & ND & $\begin{array}{l}\text { Nude mice } \\
\text { bearing human } \\
\text { colon carcinoma }\end{array}$ & [118] \\
\hline CD13 & $\begin{array}{l}\text { Cyclic NGR } \\
\text { peptide }\end{array}$ & $\begin{array}{l}\text { Paramagnetic } \\
\text { quantum dots }\end{array}$ & $\approx 30$ & 7.1 at $7.0 \mathrm{~T}$ & $\begin{array}{l}\text { Nude mice } \\
\text { bearing human } \\
\text { colon carcinoma }\end{array}$ & [119] \\
\hline $\begin{array}{l}\text { SMCe } \\
\text { tissue } \\
\text { factor }\end{array}$ & $\begin{array}{l}\text { Anti tissue } \\
\text { factor pAb }\end{array}$ & $\begin{array}{l}\text { Gd-perfluorocarbon } \\
\text { nanoparticle }\end{array}$ & $\approx 270$ & $\begin{array}{l}17.9 \text { at } 1.5 \mathrm{~T} \\
9.7 \text { at } 4.7 \mathrm{~T}\end{array}$ & $\begin{array}{l}\text { In vitro porcine } \\
\text { aortic smooth } \\
\text { muscle cells }\end{array}$ & [120] \\
\hline \multirow[t]{2}{*}{ E-selectin } & $\mathrm{H} 18 / 7 \mathrm{mAb}$ & $\begin{array}{l}\text { Paramagnetic and } \\
\text { fluorescent } \\
\text { liposomes }\end{array}$ & $\approx 120$ & $\begin{array}{l}5.5 \text { at } 1.5 \mathrm{~T} \\
5.2 \text { at } 7.0 \mathrm{~T}\end{array}$ & In vitro HUVEC & [121] \\
\hline & $\begin{array}{l}\text { Anti-human } \\
\text { E-selectin } \\
\text { fragments } \\
\mathrm{H} 18 / 7 \mathrm{~F}\left(\mathrm{ab}^{\prime}\right)_{2}\end{array}$ & $\begin{array}{l}\text { Cross-linked iron } \\
\text { oxide nanoparticles }\end{array}$ & $\approx 45$ & 48 at $0.47 \mathrm{~T}^{\mathrm{c}}$ & $\begin{array}{l}\text { Nude mice } \\
\text { implanted with } \\
\text { HUVEC in } \\
\text { Matrigel }\end{array}$ & {$[122]$} \\
\hline ICAM-1 & $\begin{array}{l}\text { Anti ICAM-1 } \\
\text { mAb }\end{array}$ & $\begin{array}{l}\text { Paramagnetic and } \\
\text { fluorescent } \\
\text { polymerized } \\
\text { liposomes }\end{array}$ & $300-350$ & ND & $\begin{array}{l}\text { Ex vivo mouse } \\
\text { brains with } \\
\text { experimental } \\
\text { autoimmune } \\
\text { encephalitis }\end{array}$ & [123] \\
\hline NCAM & $\begin{array}{l}\text { C3d peptide } \\
\text { derivative }\end{array}$ & $\begin{array}{l}\text { Gd-loaded } \\
\text { apoferritin }\end{array}$ & $12-15$ & $\begin{array}{l}\approx 70 \text { at } \\
0.47 \mathrm{~T}\end{array}$ & $\begin{array}{l}\text { Tumor ECs } \\
\text { implanted in nude } \\
\text { mice within } \\
\text { growth factor } \\
\text { reduced Matrigel } \\
\text { plugs }\end{array}$ & [124] \\
\hline
\end{tabular}

aAll values are $r_{1}$ relaxivities, unless stated otherwise. ${ }^{b}$ ND: not determined. ${ }^{c} r_{2}$ relaxivity. ${ }^{d}$ HUVEC: human umbilical vein endothelial cells. ${ }^{\text {eSMC: }}$ smooth muscle cells. Table adapted from Kiessling et al [67]. 


\section{CHAPTER 2. MRI OF VESSEL GROWTH AND FUNCTION}

A disadvantage of iron oxide particles is their rather high uptake by the spleen and the liver, which seems to render the localization of angiogenic activity in these organs impossible.

Recently, Waters et al. showed that diffusion-weighted ${ }^{19} \mathrm{~F}$ spectroscopy allowed differentiation between the signals originating from bound $\alpha_{v} \beta_{3}$-targeted perfluorocarbon (PFC) nanoparticles and unbound circulating particles in tumor bearing mice [125]. This finding potentially solves the problem that unbound particles can conceal the signal from specifically bound particles. Unfortunately, specific hardware is required for ${ }^{19} \mathrm{~F} \mathrm{MRI}$, which hampers widespread implementation.

To estimate the detection limit of molecular MRI, we calculated the theoretically achievable contrast-to-noise ratio (CNR) as a function of particle concentration (Figure 2.9). For Gd-DTPA and dendrimers with 4 or $64 \mathrm{Gd}$ ions, the obtained detection limit corresponds well with previously reported values [126]. For PFCnanoparticles and liposomes, detection limits are in the expected range of biomarker concentrations, supporting their potential applicability as molecular MRI contrast agent. For USPIO, an unexpectedly high detection limit of $\approx 50 \mu \mathrm{mol} / \mathrm{L}$ was found. This illustrates the shortcoming of the model calculation, which does not take specific particle accumulation into account, but assumes a homogeneous plasma distribution. However, USPIO aggregation will result in strong, local magnetic field inhomogeneities and an accordingly altered Larmor frequency. Consequently, proton excitation will be less efficient and reduced signal intensities will be observed. Compared with unbound USPIO, this will lead to stronger signal decreases and a lower detection limit, potentially allowing the generation of large signal differences without the high contrast agent concentrations as required for gadolinium-based contrast agents. Obviously, particle aggregation will also lower the detection limit for PFCnanoparticles and liposomes, but the effect is expected to be more pronounced for USPIO. Nevertheless, the simple model calculation presented in Figure 2.9 may be helpful to predict if a particle is a potentially suitable contrast agent for $T_{1}$-weighted molecular MRI.

Presently, molecular MRI experiments have been performed only in animals because no targeted contrast agent has been approved for clinical use. In contrast, several of the carrier particles in Table 2.2 were applied for other therapeutic or imaging applications, such as liposomal formulations carrying cytostatic drugs and non-targeted iron oxide particles for liver metastasis and lymph node imaging. It is therefore expected that these particles are more likely to become clinically approved molecular MRI contrast agents, although many problems still have to be solved. For instance, particles need to be synthesized according to good laboratory practice and have to sustain extensive safety and efficacy testing. Also, stronger signal amplification strategies, more sensitive imaging protocols with a high spatial resolution, and adequate post-processing software will be required. Finally, thorough validation and reproducibility studies are required. 


\section{Cellular imaging}

MRI can also be applied to study vasculogenesis. Arbab et al. showed that the migration and incorporation of iron oxide-labeled EPCs into the tumor vasculature in mice could be accurately detected using in vivo MRI [127]. Immunohistochemical analysis demonstrated that EPCs were transformed into ECs, indicating that iron oxide labeling does not interfere with EPC differentiation. Additionally, Weber et al. showed that iron oxide-labeled EPCs could also be detected ex vivo in ischemic rat hearts [128]. Furthermore, ${ }^{19} \mathrm{~F}$ imaging and spectroscopy was recently applied to study PFC-labeled progenitor cells both in vitro and in vivo [129].

\subsection{Clinical considerations and future directions}

\section{Contrast agent safety}

Although gadolinium-based contrast agents are considered to be relatively safe owing to the absence of radiation, a high chemical inertness, and rapid renal clearance, recent evidence showed that these agents potentially cause nephrogenic systemic fibrosis (NSF) in patients with severe renal impairment. Although the relative risk of developing NSF is low and appears to be different for the various gadoliniumcontaining agents, the FDA issued a warning for all agents regarding their use in patients with renal insufficiency (glomerular filtration rate $<30 \mathrm{~mL} / \mathrm{min} / 1.73 \mathrm{~m}^{2}$ ) $[130,131]$.

For blood pool agents, clinical implementation is hampered by potential toxicity from incomplete gadolinium clearance. Due to their size, macromolecular contrast agents generally have to be metabolized before they can be cleared via renal filtration, which may increase the risk of cellular uptake and consequent gadolinium release [132]. Additionally, macromolecular contrast agents may invoke an immune response. The potential clinical evaluation of molecular MRI is further hampered by the large doses of contrast agent that are currently required, as demonstrated in animal studies, and the fact that possible detrimental health effects are largely unknown.

Currently, three potential solutions to increase the renal clearance of macromolecular agents exist. First, medium-sized agents, such as the dendritic agent Gadomer, are being developed which have a molecular weight smaller than the renal filtration cutoff of approximately $20 \mathrm{kDa}$ [132]. Second, small agents might be used which bind reversibly to plasma proteins such as albumin. An example is Vasovist, which is already clinically approved in Europe. Third, biodegradable polymers are under development, which have a relatively high renal excretion rate and show minimal gadolinium accumulation [132]. 


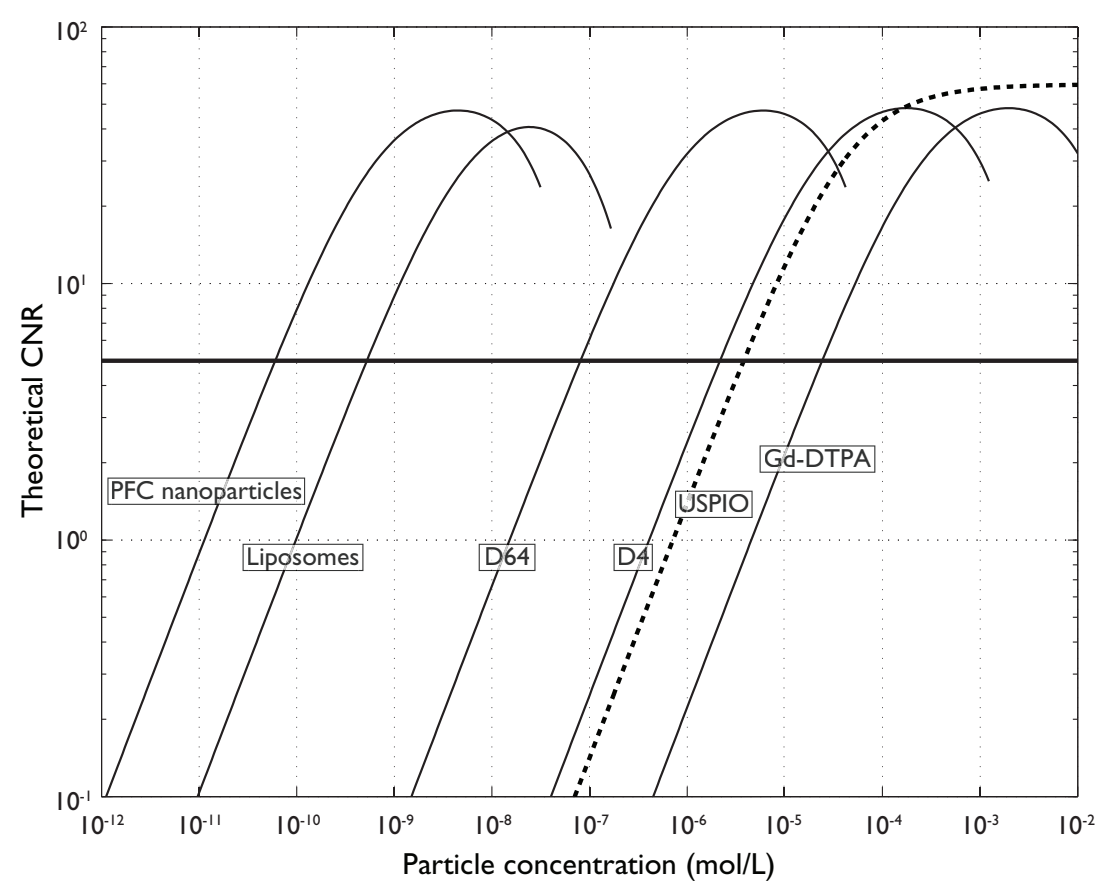

Figure 2.9: Theoretical contrast-to-noise ratio (CNR) versus particle concentration for PFCnanoparticles, liposomes, dendrimers containing 64 (D64) or 4 Gd ions (D4), USPIO and Gd-DTPA. For gadolinium- or iron-based particles, a spin echo or gradient echo sequence was assumed, respectively. For clarity, absolute values are plotted for USPIO (dotted line) due to the inherent negative CNR. The minimally detectable CNR is considered to be 5 (solid horizontal line). The detection limit for each particle is found at the corresponding intercept. Required particle relaxivities were obtained from references provided in Table 2.2 and from ref [126].

\section{High field strength}

Currently, there is trend towards MRI at higher magnetic field strengths $\left(B_{0}\right)$. Although for clinical systems $1.5 \mathrm{~T}$ is still standard, $3 \mathrm{~T}$ is becoming more prevalent, particularly for brain MRI, and scanners operating at $7 \mathrm{~T}$ and $9.4 \mathrm{~T}$ are already available in a number of centers [133]. For dedicated animal systems, field strengths of $7 \mathrm{~T}$ and higher prevail. With this in mind, several remarks considering MRI of neovascularization can be made. First, stronger magnetic fields offer better spatial resolution and SNR. It is expected that this will bring the detection limit for blood vessels back to a caliber of approximately $200 \mu \mathrm{m}$ for humans and $50 \mu \mathrm{m}$ for mice. Furthermore, $T_{2}^{*}$ decreases with increasing field strength, which makes susceptibility based methods like VSI more sensitive. However, susceptibility artifacts are also enhanced, and field homogeneity and shimming become more important at higher field strength. In contrast, $T_{1}$ increases with increasing $B_{0}$, which results in a reduction of inherent tissue contrast. 


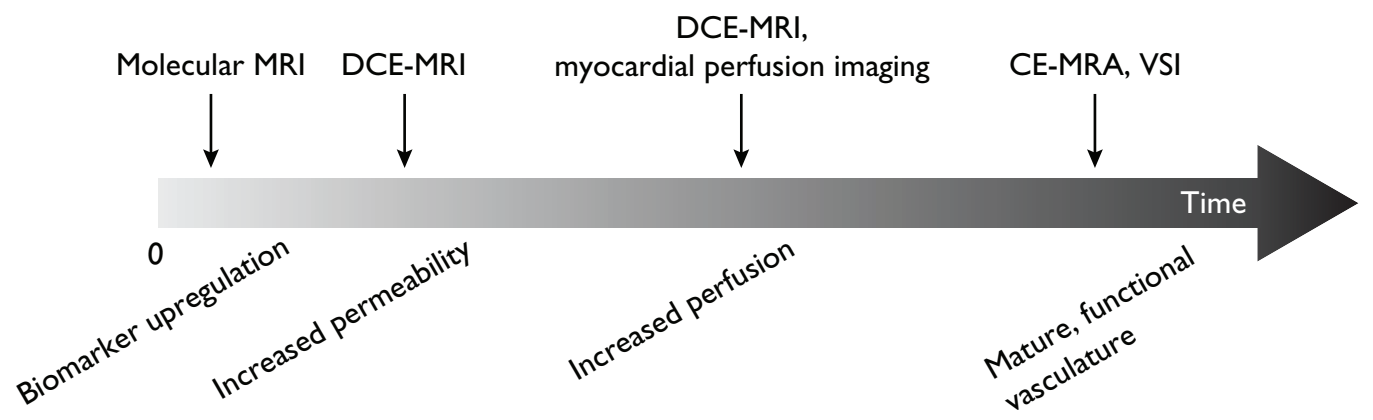

Figure 2.10: Schematic representation of the time course of neovascularization showing suitable contrast-enhanced MRI techniques that may be employed to investigate vessel growth at different scales and time-points.

An apparent problem of higher $B_{0}$ is the reduced relaxivity of positive contrast agents [134]. Combined with the increased $T_{1}$, contrast-enhanced methods are considerably less sensitive at higher magnetic fields. However, this may be partially compensated by a decrease in partial volume effect due to the increase in spatial resolution and/or SNR. The contrast enhancement of negative contrast agents will likely improve due to the increased sensitivity to field inhomogeneities at increasing $B_{0}$. It is therefore expected that negative contrast agents will become more important for imaging of the neovasculature if the tendency towards higher $B_{0}$ continues.

A final concern for imaging at higher field strengths, especially when using gradient echo sequences, are strong image artifacts caused by local changes in magnetic field susceptibility and altered blood flow profiles due to non-natural objects such as stents or artificial heart valves. However, when the foreign object resides outside the field of interest, these artifacts are not considered a major problem for image quality.

\subsection{Conclusions}

In this review, we have shown that contrast-enhanced MRI is suitable for imaging of both the established and the developing vasculature at morphological, functional, and molecular levels. The ability to detect these diverse aspects of neovascularization makes contrast-enhanced MRI in theory attractive to follow the entire time course of neovascularization, from the first onset until the establishment of a functional mature vasculature (Figure 2.10). Clinically, contrast-enhanced MRI may be applied to monitor patient response after pro- or antiangiogenic treatments.

A comprehensive overview of the presented MRI techniques is provided in Table 2.3. It was shown that CE-MRA enables morphological characterization and that small vessels with diameters even smaller than the imaging voxel size may be detected using VSI. Furthermore, DCE-MRI can assess several functional parameters of neovascularization, such as vessel permeability, flow, and perfusion. 
Table 2.3: Summary of the presented contrast-enhanced MRI techniques. Advantages, disadvantages, and the vessel type to which the techniques can be applied are given.

\begin{tabular}{|c|c|c|c|}
\hline $\begin{array}{l}\text { MRI } \\
\text { technique }\end{array}$ & Advantages & Disadvantages & Vessel type \\
\hline CE-MRA & $\begin{array}{l}\text { High vessel to background contrast; } \\
\text { short acquisition time; no blood } \\
\text { saturation artifacts }\end{array}$ & $\begin{array}{l}\text { Requires acurate bolus timing; depiction of } \\
\text { small vessel ( }<0.5 \mathrm{~mm} \text { ) problematic; } \\
\text { non-specific tissue contrast due to } \\
\text { extravasation of small contrast agents }\end{array}$ & $\begin{array}{l}\text { Mature vessels; } \\
\text { macrocirculation; arteries } \\
\text { and veins }\end{array}$ \\
\hline $\begin{array}{l}\text { Vessel size } \\
\text { imaging }\end{array}$ & $\begin{array}{l}\text { Quantification of vessel size and } \\
\text { blood volume }\end{array}$ & $\begin{array}{l}\text { Sensitive to susceptibility effects; requires } \\
\text { additional diffusion measurement; no direct } \\
\text { visualization, mathematical analysis required }\end{array}$ & $\begin{array}{l}\text { Mature and developing } \\
\text { vessels; micro- and } \\
\text { macrocirculation; arteries } \\
\text { and veins }\end{array}$ \\
\hline DCE-MRI & $\begin{array}{l}\text { High versatility; estimation of } \\
\text { contrast agent pharmacokinetics; } \\
\text { sensitive to tumor aggressiveness; } \\
\text { applied in many preclinical and clinical } \\
\text { studies }\end{array}$ & $\begin{array}{l}\text { Requires high temporal resolution; often low } \\
\text { spatial resolution and/or anatomical } \\
\text { coverage; no consensus on data analysis; } T_{2}^{*} \\
\text { effects at high magnetic fields; no direct } \\
\text { visualization, mathematical optimization } \\
\text { required; moderate reproducibility; no } \\
\text { suitable validation }\end{array}$ & $\begin{array}{l}\text { Developing vessels; } \\
\text { microcirculation; arteries } \\
\text { and veins }\end{array}$ \\
\hline $\begin{array}{l}\text { Myocardial } \\
\text { perfusion } \\
\text { imaging }\end{array}$ & $\begin{array}{l}\text { Absolute quantification of myocardial } \\
\text { blood flow; perfusion reserve sensitive } \\
\text { to the severity of coronary artery } \\
\text { stenosis }\end{array}$ & $\begin{array}{l}\text { No direct visualization, mathematical } \\
\text { analysis required; not applicable in } \\
\text { preclinical research with rodents }\end{array}$ & $\begin{array}{l}\text { Mature and developing } \\
\text { vessels; micro- and } \\
\text { macrocirculation; } \\
\text { arteries; collaterals }\end{array}$ \\
\hline $\begin{array}{l}\text { Molecular } \\
\text { MRI }\end{array}$ & $\begin{array}{l}\text { Potential disease specificity; possible } \\
\text { earlier diagnosis; non-invasive } \\
\text { monitoring of pro- or antiangiogenic } \\
\text { treatments }\end{array}$ & $\begin{array}{l}\text { Low target concentration; requires high } \\
\text { local contrast agent concentration; } \\
\text { unknown sensitivity, specificity, and toxicity; } \\
{ }^{19} \text { F imaging requires special hardware; } \\
\text { currently not clinically applicable }\end{array}$ & $\begin{array}{l}\text { Developing vessels; } \\
\text { microcirculation; arteries } \\
\text { and veins }\end{array}$ \\
\hline
\end{tabular}


In preclinical research, MRI may be valuable to solve basic questions regarding blood vessel development and function, using, for instance, a combination of morphological, functional, and/or molecular methods. Furthermore, molecular MRI may be valuable to differentiate between the two forms of neovascularization, provided that specific markers for these two processes become available.

In conclusion, considering the versatility in available MRI methods and the exciting new developments in scanner hardware, pulse sequences, post-processing, and contrast agents (i.e. targeted and/or macromolecular), it is expected that contrastenhanced MRI will further grow in importance to address advanced scientific and/or clinical needs concerning vessel growth and to visualize vessels at a submillimeter resolution. 



\section{Part I}

Tumor microvasculature 



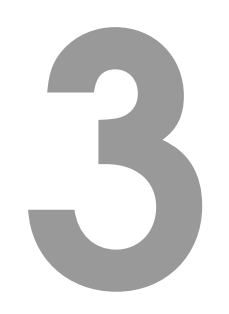

\section{Quantitative molecular MRI of tumor angiogenesis using cNGR-labeled paramagnetic quantum dots}

The objective of this study was to develop and apply cyclic Asn-Gly-Arg-labeled paramagnetic quantum dots (cNGR-pQDs) for the non-invasive assessment of tumor angiogenic activity using quantitative in vivo molecular magnetic resonance imaging (MRI). cNGR was previously shown to colocalize with CD13, an aminopeptidase that is highly overexpressed on angiogenic tumor endothelium. Since angiogenesis is important for tumor growth and metastatization, its in vivo detection and quantification may allow objective diagnosis of tumor status and evaluation of treatment response.

Intravenous injection of cNGR-pQDs in tumor-bearing mice resulted in increased quantitative contrast, comprising increased longitudinal relaxation rate and decreased proton visibility, in the tumor rim but not in tumor core or muscle tissue. This demonstrated that cNGR-pQDs allow in vivo quantification and accurate localization of angiogenic activity. MRI results were validated using ex vivo two-photon laser scanning microscopy (TPLSM), which showed that cNGR-pQDs were primarily located on the surface of tumor endothelial cells and to a lesser extent in the vessel lumen. In contrast, unlabeled $\mathrm{pQDs}$ were not or only sparsely detected with both MRI and TPLSM, supporting a high specificity of cNGR-pQDs for the angiogenic tumor vasculature.

Cancer Research. 2008; 68: 7676-83 


\subsection{Introduction}

Angiogenesis, the formation of new capillaries from existing blood vessels, is key to tumor growth and metastatization by providing proliferating tumor cells with oxygen and nutrients $[2,135]$. Moreover, angiogenic activity is related to tumor malignancy $[3,92]$. Non-invasive detection of angiogenic activity is therefore highly relevant for adequate tumor diagnosis. Quantification of angiogenesis may furthermore allow objective monitoring of tumor progression, for instance in response to treatment.

Currently, molecular imaging techniques are being developed that allow direct visualization and characterization of cellular or molecular activation of angiogenesisrelated pathways [108]. More specifically, molecular imaging uses contrast agents that home to upregulated biomolecules (e.g. receptors, enzymes) via interaction with high-affinity ligands coupled to the contrast agent. Ideally, this results in an altered signal intensity at the location of these molecules. Of the different imaging modalities, magnetic resonance imaging (MRI) may be the most desirable for molecular imaging due to its excellent spatial resolution and soft tissue contrast. Moreover, molecular MRI potentially allows direct covisualization of tumor angiogenic activity with anatomy. However, the inherently low sensitivity of MRI is a problem due to the typically low abundance of upregulated biomolecules. This can be overcome by using large molecular weight constructs carrying a high payload of gadolinium or iron, and multiple targeting ligands to enhance the particle's relaxivity and targeting efficacy, respectively [19].

One of the best-defined ligands for molecular imaging of angiogenesis is the cyclic Arg-Gly-Asp (cRGD) peptide, which binds specifically to the $\alpha_{\mathrm{v}} \beta_{3}$-integrin $[110,113]$. However, for the cyclic Asn-Gly-Arg (cNGR) motif, the tumor-homing capability was shown to be 3-fold higher compared with CRGD [136]. The clinical applicability of cNGR as a tumor-homing ligand was previously demonstrated by conjugating cNGR to tumor necrosis factor $\alpha$ (TNF $\alpha)$. Compared with unlabeled TNF $\alpha$, cNGRTNF $\alpha$ displayed a significantly increased anti-tumor activity with similar systemic toxicity [137-139].

The vascular address of cNGR is a specific isoform of CD13 (aminopeptidase N), a transmembrane glycoprotein involved in cancer angiogenesis, tumor invasion, and metastasis, which is overexpressed by activated endothelial cells (ECs) of tumor vasculature $[136,140,141]$. CD13 is not required for vessel growth during embryonic development and normal adult function, as shown in CD13-null mice [142]. In a model of retinal neovascularization, these mice had significantly decreased vessel growth, suggesting that CD13 is important in pathological neovascularization. In addition, fluorophore-conjugated cNGR allowed detection of the in vivo expression of CD13 in tumors and in infarcted myocardium $[143,144]$. Competition with unconjugated cNGR significantly decreased the fluorescence signal, indicating high specificity of cNGR for CD13 [143, 144]. 
Despite the aforementioned high tumor-homing capability of cNGR, its potency as a targeting ligand for molecular imaging of tumor angiogenesis is currently unknown. Therefore, the objective of this study was to explore cNGR-labeled paramagnetic quantum dots (cNGR-pQDs) for the non-invasive and selective in vivo detection of tumor neovascularization using quantitative molecular MRI. QDs were chosen as contrast agent scaffolds because of their excellent photophysical properties, i.e. broad excitation, small emission spectra, and limited photo-bleaching $[145,146]$. Furthermore, QDs enabled binding of multiple targeting ligands and gadolinium chelates. The particle's bimodal nature (i.e. paramagnetic and fluorescent) allowed validation of the results with ex vivo two-photon laser scanning microscopy (TPLSM). With TPLSM, three-dimensional contrast agent localization can be obtained at subcellular resolution with a penetration depth reaching $250 \mu \mathrm{m}$ in tumors.

MRI data were analyzed via absolute quantification of contrast agent induced changes in the tissue's longitudinal relaxation rate $R_{1}\left(1 / T_{1}\right)$, which is proportional to contrast agent concentration, and proton visibility. The latter expectedly decreases at high densities of paramagnetic contrast material. Quantitative analysis requires acquisition of a series of images and may provide improved sensitivity in molecular MRI. Theoretically, the employed inversion recovery (IR) technique has an inherent two-fold higher sensitivity than spin echo pulse sequences and by measuring a series of images it potentially allows detection of smaller changes in $R_{1}$ than a single image.

Both MRI and TPLSM showed specific binding of cNGR-pQDs to ECs in the angiogenic tumor rim, but not in tumor core or muscle tissue. Furthermore, a significantly lower quantitative contrast was found with unlabeled pQDs, indicating a high specificity of the cNGR-labeled contrast agent for angiogenic ECs. To our knowledge, this is the first non-invasive in vivo application of cNGR as a targeting ligand for molecular MRI of tumor angiogenesis.

\subsection{Methods}

\section{Preparation of cNGR-labeled paramagnetic quantum dots}

NAc-Cys(4MeBzl)-Asn(Xanthyl)-Gly-Arg(Tosyl)-Cys(4MeBzl)-Gly-Gly-Lys (Fmoc)peptide was synthesized by $\mathrm{tBoc}$ solid phase peptide synthesis, as described previously $[143,147]$. On the resin, the lysine side chain was selectively deprotected by treatment with $20 \%$ piperidine/dimethylformamide $(4 \times 3 \mathrm{~min})$. After covalent coupling of biotin-succinimidyl ester (Molecular Probes, Eugene, OR) to the lysine $\epsilon$-amino group to obtain biotinylated peptide-resin, the peptide was deprotected and cleaved from the resin using anhydrous hydrogen fluoride for $1 \mathrm{~h}$ at $0{ }^{\circ} \mathrm{C}$ with $4 \% \mathrm{p}$ cresol as scavenger and lyophilized. Electrospray ionization mass spectrometry (ESIMS) revealed a mass of 1287.4, corresponding well to the theoretical average mass (1288.7) of the reduced biotinylated Nac-Cys-Asn-Gly-Arg-Cys-Gly-Gly-Lys(biotin)- 
$\mathrm{NH}_{2}$ peptide. Oxidative folding of the crude product in $0.1 \mathrm{M}$ Tris, $\mathrm{pH} 8,1 \mathrm{M}$ guanidin at $4{ }^{\circ} \mathrm{C}$ for 16 hours yielded the internal disulfide bridged biotin-cyclic NGR, which was HPLC-purified (C18 RP-HPLC) and lyophilized. ESI-MS confirmed a mass decrease of 2 , representing the loss of 2 protons from the cystein side chains due to the generation of 1 disulfide bond (S-S). Biotinylated poly (lysine) dendritic wedge, a construct comprising 8 Gd-DTPA moieties, was synthesized and purified similarly $[148,149]$.

Curnis et al. previously showed that cNGR spontaneously converts into isoDGR by asparagine deamidation at slightly basic $\mathrm{pH}$, generating an $\alpha_{\mathrm{v}} \beta_{3}$-integrin ligand [150]. Using a combination of HPLC and mass spectrometry upto 24 hours after dissolving $\mathrm{cNGR}$ in water ( $\mathrm{pH}$ 6.0) or in $1 \mu \mathrm{M}$ borate buffer ( $\mathrm{pH}$ 8.3, supplemented with $0.05 \% \mathrm{NaN}_{3}$ ), it was found that this process did not occur in the time-period of the experiments (data not shown).

Bimodal, multivalent contrast agent was prepared as follows. Streptavidin conjugated QDs (1 $\mu \mathrm{M}$ in borate buffer $\mathrm{pH}$ 8.3, emission at $585 \mathrm{~nm})$ were purchased from Invitrogen (Breda, The Netherlands). QDs were composed of a CdSe core with a ZnS shell and covered with polyethyleneglycol-2000. Each QD holds approximately 10 surface-bound streptavidins, allowing 30 biotinylated compounds to bind on average (personal communication with Invitrogen). For each experiment, cNGRpQDs were prepared freshly at room temperature by sequential mixing of $100 \mu \mathrm{LD}$ solution with biotin-cNGR and biotin-poly(lysine) dendritic wedge, both dissolved in Hanks balanced salt solution (HBSS, pH 7.4, Invitrogen), in a molar ratio of 1:6:24 to a total volume of approximately $120 \mu \mathrm{L}$. Samples were mildly vortexed during each preparation step to ensure a homogeneous distribution of biotin-cNGR and biotin-poly(lysine) dendritic wedge over the QD surface. Overall, each QD carried a maximum of $192 \mathrm{Gd}$ ions and 6 cNGR peptides. Unlabeled pQDs carried the same number of $\mathrm{Gd}$ constructs but no cNGR. A schematic representation of the cNGR-pQD particle is shown in Figure 3.1.

\section{Animal model}

All animal studies were approved by the institutional animal welfare committee. $1.5-$ $3 \times 10^{6}$ human colorectal adenocarcinoma cells (LS174T, American Type Culture Collection CL-188, Rockville, MD) were subcutaneously and unilaterally injected on the flank of 15 week old male Swiss nu/nu mice (Charles River, Maastricht, The Netherlands). Mice were subjected to the MRI examination when the tumor diameter was $>1.0 \mathrm{~cm}$, which was approximately 16 days after LS174T injection.

For in vivo MRI, mice were anesthetized using $1.5-2.0 \%$ isoflurane (Abbott Laboratories Ltd, Queensborough, UK) in medical air and were placed prone in a dedicated animal holder with built-in mask for anesthesia gas supply. An infusion line was placed in the jugular vein for contrast agent administration during the MRI experiment. A heating pad was placed over the mice to maintain normothermic 


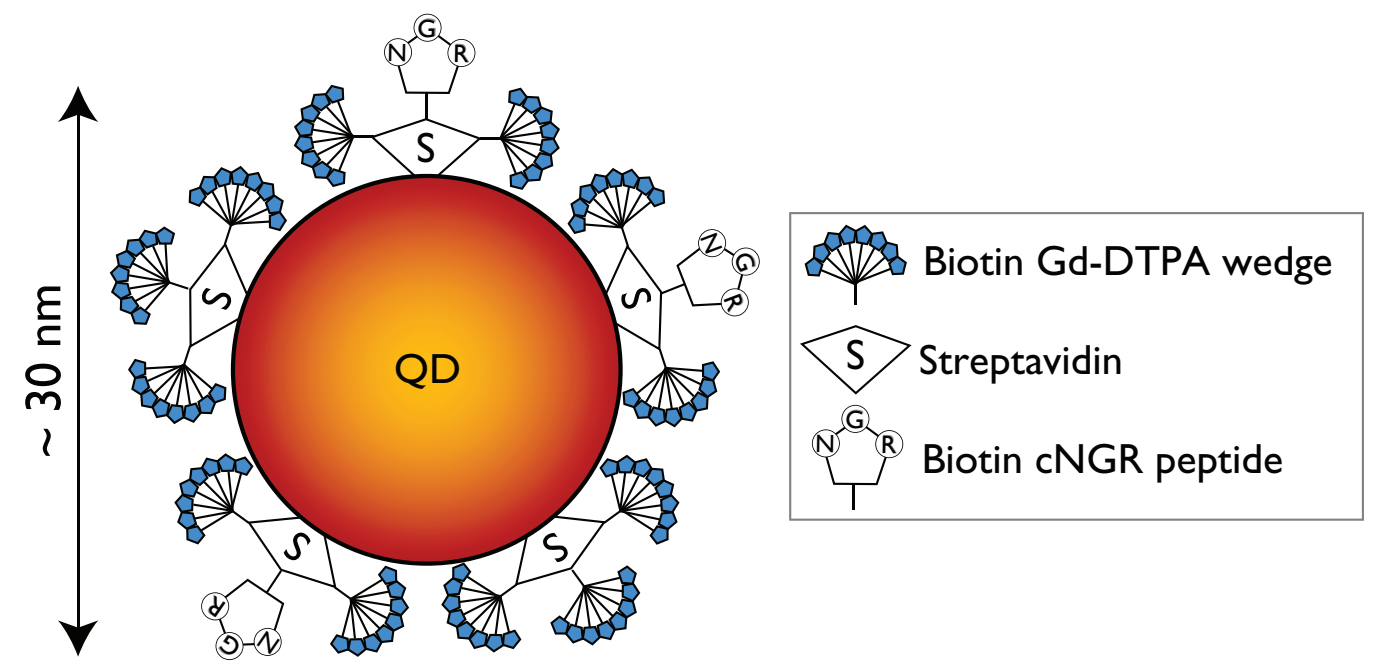

Figure 3.1: Schematic representation of a cNGR-labeled paramagnetic quantum dot. Each particle carries approximately 10 streptavidin moieties to which 6 cNGR groups and 24 dendritic gadolinium constructs were bound. The total number of gadolinium ions per particle was maximally 192. Particle size was measured using dynamic light scattering (Chapter 4).

conditions. Respiration rate and body temperature were continuously monitored via a balloon sensor and rectal temperature probe, respectively, interfaced to an MR compatible small animal monitoring system (SA Instruments Inc, Stony Brook, NY).

Mice were randomly selected for injection with either cNGR-pQDs or unlabeled pQDs. Seven mice were included for each contrast agent group. Mice were kept inside the magnet during the entire MRI experiment to preserve their position.

\section{MRI protocol}

All MRI experiments were performed on a 7 Tesla Bruker Biospec 70/30 USR MRI system, interfaced to an AVANCE II console (Bruker Biospin GmbH, Ettlingen, Germany). The BGA12-S mini imaging gradient (maximum gradient strength $720 \mathrm{mTm}^{-1}$, slew rate $6000 \mathrm{Tm}^{-1} \mathrm{~s}^{-1}$ ) and a $3.5 \mathrm{~cm}$ inner diameter quadrature volume resonator were used.

\section{Molecular MRI}

Tumors were localized using $T_{2}$-weighted anatomical images (TR 4200, TE $37.4 \mathrm{ms).}$ Next, pre-contrast $R_{1}$ values were determined using a series of IR measurements with increasing inversion times (TR 4000, TE 8.4, TI 500, 1000, 1500, 2000, 2500 and $3500 \mathrm{~ms}$; total scan time 18 minutes). Subsequently, mice were injected with $120 \mu \mathrm{L}$ of cNGR-pQDs or unlabeled pQDs, followed by a $50 \mu \mathrm{L}$ saline flush. IR experiments 
were repeated approximately 30 minutes post-contrast to ensure adequate contrast agent circulation and a reduced level of intravascular contrast agent. Images were recorded using a field of view (FOV) of $4.0 \times 4.0 \mathrm{~cm}^{2}$, a $192 \times 192$ acquisition matrix interpolated to $256 \times 256$ by means of zero-filling, and a slice thickness of $1.2 \mathrm{~mm}$, resulting in $0.16 \times 0.16 \times 1.2 \mathrm{~mm}^{3}$ sized voxels. On average, 15 contiguous slices were recorded in multi-slice mode (range $11-22$ slices, depending on tumor size and orientation). After MRI, mice were euthanized by cervical dislocation.

\section{Competition experiment}

Four tumor-bearing mice were randomly selected for a competition experiment of cNGR-pQDs with unconjugated cNGR, i.e. non-biotinylated, non-paramagnetic, and non-fluorescent. Imaging was performed as described above, except that $525 \mu \mathrm{g}$ per mouse of unconjugated cNGR, i.e. a 1000-fold excess as compared to QD-bound cNGR, was injected intravenously 10 minutes after administration of cNGR-pQDs.

\section{Biodistribution}

Healthy Swiss mice (Charles River) were injected with either cNGR-pQDs, unlabeled pQDs, or no contrast agent. After approximately 1 hour circulation time, mice were sacrificed and whole body $T_{1}$-weighted spin echo images were recorded (TR 1100, TE $8.5 \mathrm{~ms}$, FOV $4.0 \times 6.0 \mathrm{~cm}^{2}$, matrix $256 \times 512$, resolution $0.16 \times 0.12 \times 1.2 \mathrm{~mm}^{3}$ ). Two mice were included per group.

\section{Tissue harvesting}

After MRI, tumor, spleen, liver, kidney, hind limb muscle, heart, and lung were excised and embedded in optimal cutting temperature (OCT) compound (Sakura Finetek Europe, Zoeterwoude, The Netherlands). Next, tissues were snap-frozen in cold 2-methylbutane (Acros Organics, Geel, Belgium) for approximately 2 minutes and subsequently transferred to liquid nitrogen. Tissues were stored at $-80{ }^{\circ} \mathrm{C}$ until TPLSM measurements.

\section{Contrast agent relaxivity}

$T_{1}$ relaxivity $\left(r_{1}\right)$ was determined by diluting cNGR-pQDs in HBSS in 9 steps to concentrations of $0-0.001 \mathrm{mM}$ (corresponding gadolinium concentrations: 0 $0.192 \mathrm{mM}$ ). The $R_{1}$ of each sample was determined using the IR series as described above. Absolute gadolinium concentrations were measured using inductively coupled plasma mass spectrometry. Longitudinal relaxivity was determined by the slope of a linear fit of $R_{1}$ versus gadolinium concentration. 


\section{MRI data analysis}

All data processing was performed in Matlab (The Mathworks, Natick, MA), unless stated otherwise. IR images were first spatially coregistered using the mutual information algorithm in the MIRIT software package [151] to correct for possible animal motion in the images with different $T_{1}$ contrast, and smoothed with a three dimensional Gaussian kernel with a full-width-at-half-maximum of $0.4 \times 0.4 \times 3.0 \mathrm{~mm}^{3}$. Regions of interest (ROIs) were drawn manually in MRIcro [152] to define tumor and muscle tissue. Both $T_{1}$ - and $T_{2}$-weighted images were used to accurately delineate tumors from surrounding tissue and edema. Pre- and post-contrast $R_{1}$ values were determined on a voxel-by-voxel basis by non-linear curve fitting of the IR signal intensity function [153]:

$$
S=S_{0}\left(1-2 e^{-T I \cdot R_{1}}+e^{-T R \cdot R_{1}}\right)
$$

using the Levenberg-Marquardt optimization algorithm. $S_{0}$ is a scaling factor including proton density, excitation pulse profile, echo time, and pre-amplifier gain. The detection limit for changes in $R_{1}\left(\Delta R_{1}=R_{1, \text { post }}-R_{1 \text {,pre }}\right)$ was determined with a Monte Carlo simulation using Equation 3.1, in vivo relaxation rates, and representative noise levels as derived from the in vivo experiments. A voxel was considered significantly enhanced when $\Delta R_{1}$ was more than 1.96 (i.e. $95 \%$ confidence interval) times higher than the detection limit of $0.005 \mathrm{~s}^{-1}$. We defined the quantitative contrast derived from the $\Delta R_{1}$ measurements $\left(Q C_{R_{1}}\right)$ as the product of the mean $\Delta R_{1}$ and the percentage of significantly enhanced voxels for each tissue type, i.e. tumor rim and core, and muscle tissue. $Q C_{R_{1}}$ indicates both the level and spatial extent of contrast agent binding. Changes in $S_{0}\left(\triangle S_{0}=S_{0, \text { post }}-S_{0 \text {,pre }}\right)$ were also evaluated and the quantitative contrast from $S_{0}\left(Q C_{S_{0}}\right)$ was defined analogously to $Q C_{R_{1}}$ to yield a quantity that reflects proton visibility [154].

\section{Tumor rim/core analysis}

To investigate the differences between tumor rim, i.e. the region with the highest expected angiogenic activity, and core, the tumor rim was first defined as an approximately $1 \mathrm{~mm}$ thick peripheral zone with the strongest $R_{1}$ enhancement, in accordance with the approach taken by others $[81,155]$. The rim comprised $29.0 \pm 5.8 \%$ and $31.6 \pm 3.5 \%$ of all tumor voxels for mice injected with cNGR-pQDs and unlabeled pQDs, respectively. The tumor core was defined as the difference between whole tumor and tumor rim ROls. Secondly, a contour was drawn to calculate the number of voxels with a significantly increased $\Delta R_{1}$ as a function of the distance to the tumor rim. As an empiric measure of spatial heterogeneity in angiogenic tumor activity, the half-value-depth was defined as the distance from the rim at which the percentage of enhanced voxels has decreased by 50\% compared to its value at zero distance, i.e. the rim. The half-value-depth was calculated by fitting the group-averaged data presented in Figure 3.3C with a mono-exponential decay function. 


\section{Biodistribution}

ROls defining the spleen, liver, kidney, heart, lung, and aorta were drawn manually in MRIcro. Signal intensities were averaged over the entire tissue and normalized to hind limb muscle.

\section{Statistical analysis}

Statistical analysis of paired samples was performed using a non-parametric Wilcoxon signed ranks test in SPSS 14.0 (SPSS, Chicago, III). As both $Q C_{R_{1}}$ and $Q C_{S_{0}}$ represent contrast agent presence, $Q C_{R_{1}}$ and $Q C_{S_{0}}$ were combined to a summary value according to O'Brien and Läuter $[156,157]$, which is more sensitive to contrast effects than the individual measures. Therefore, $Q C_{R_{1}}$ and $Q C_{S_{0}}$ were first standardized by $z=(Q C$-mean $(Q C) / s d(Q C))$. Subsequently, the absolute values of $z_{Q C_{R_{1}}}$ and $z_{Q C_{S_{0}}}$ were averaged per animal. The resulting summary measure was tested using a nonparametric Mann-Whitney $\mathrm{U}$-test. $\mathrm{P}<0.05$ was considered statistically significant.

\section{TPLSM data acquisition}

Tissue samples were thawed and washed with HBSS to remove OCT compound. Except for the spleen and liver, tissues were incubated with 25-fold diluted $\alpha$ CD31-FITC (0.5 mg/mL, BD Biosciences Pharmingen, Alphen aan de Rijn, The Netherlands) to fluorescently label ECs. Next, tissues were embedded in $2 \mathrm{w} \%$ agarose gel (Invitrogen), with their rim upwards. For measurements in the tumor core, tumors were cut transversally to resemble the slice orientation of the MRI measurements.

TPLSM imaging was performed using a Nikon Eclipse E600FN upright microscope (Tokyo, Japan), incorporated in the Bio-Rad Radiance 2100MP imaging system, and operated by Lasersharp2000 V6.0 (Bio-Rad, Hemel Hempstead, UK). Tissue samples were excited by the Tsunami Ti:sapphire laser (Spectra-Physics, Mountain View, CA), which was pumped by a Millennia Vs $5 \mathrm{~W}$ pump laser (SpectraPhysics), and mode-locked at $800 \mathrm{~nm}$, with a $82.5 \mathrm{MHz}$ repetition rate and 100 fs pulse width. Tissues were observed through a water dipping $60 \times$ fluor objective with a 1.00 numerical aperture (Nikon). Photomultiplier tubes (PMTs $9108 \mathrm{~B} 02$ and 9136B05, Electron Tubes Limited, Ruislip, UK) were used to acquire fluorescence photons in three spectral regions: $420-470 \mathrm{~nm}$ (autofluorescence), 520-560 nm (FITC), and 570-600 nm (QDs). Each PMT was tuned for minimal bleed-through of the fluorescent markers to adjacent PMTs. Images, color-coded blue, green, and red, respectively, were subsequently merged into a single image. The in-plane pixel dwell time was $11.8 \mu \mathrm{s}$, which, together with a 2-fold Kalman averaging, resulted in an imaging speed of $0.16 \mathrm{~Hz}$. The FOV was $179 \times 179 \mu \mathrm{m}^{2}$ with a matrix size of $512 \times 512$, resulting in $0.35 \times 0.35 \mu \mathrm{m}^{2}$ sized pixels. 


\section{TPLSM data analysis}

Data were analyzed with Image-Pro Plus 6.0 (MediaCybernetics, Silver Spring, MD) and Image 1.35 ( $\mathrm{NIH}$, Bethesda, MD). Image quality was improved by convolution with a $1.05 \times 1.05 \mu \mathrm{m}^{2}$ Gaussian filter. Spatial distribution of $\mathrm{pQDs}$ was classified into four groups: intravascular, intracellular, colocalized with the EC membrane, or extravasated to the interstitium.

\subsection{Results}

\section{In vivo targeting of activated tumor endothelium}

The ability of cNGR to target angiogenic tumor ECs was evaluated in tumor-bearing nude mice by injecting them with cNGR-pQDs or unlabeled pQDs. Tumor volumes of cNGR and control groups did not differ on MR images (mean \pm SD: $1.0 \pm 0.7$ $\mathrm{cm}^{3}$ and $1.0 \pm 0.6 \mathrm{~cm}^{3}$, respectively).

For both cNGR-pQDs and unlabeled $\mathrm{pQDs}$, changes in $R_{1}\left(\Delta R_{1}\right)$ were spatially heterogeneous throughout the tumor and were most pronounced at the tumor rim (Figure 3.2A). Averaged over all mice, the $\Delta R_{1}$ induced by cNGR-pQDs ranged up to approximately $0.3 \mathrm{~s}^{-1}$, which was considerably larger than the intrinsic variation in pre-contrast tumor $R_{1}$ of $0.1 \mathrm{~s}^{-1}$. Furthermore, the range in $\Delta R_{1}$ was relatively large compared with the pre-contrast tumor $R_{1}$ of $0.8 \mathrm{~s}^{-1}$. Administration of unlabeled $\mathrm{pQD}$ s resulted in a 3-fold lower response range $\left(\Delta R_{1}<0.1 \mathrm{~s}^{-1}\right)$ compared with cNGR-pQDs.

Subsequent investigation by TPLSM allowed localization of cNGR-pQDs and unlabeled pQDs at a subcellular resolution. cNGR-pQDs were found to colocalize approximately three times more often with tumor ECs than unlabeled pQDs (Figure 3.2). cNGR-pQDs and unlabeled pQDs were also found in the vessel lumen, albeit that cNGR-pQDs were approximately three times more prevalent than unlabeled $\mathrm{pQDs}$. Both contrast agents were only sparsely found to have extravasated into the tumor interstitium. Although cNGR was previously reported to be an internalizing peptide [158], cNGR-pQDs were not detected inside ECs with TPLSM.

Further evidence for the specificity of cNGR was provided by $\Delta R_{1}$ in hind limb muscle. Here, average $\Delta R_{1}$ upon administration of cNGR-pQDs was considerably lower than in the tumor and ranged up to $0.05 \mathrm{~s}^{-1}$. TPLSM did not display colocalization of cNGR-pQD with ECs of muscle vasculature. However, the incidence of cNGR-pQDs in the muscle vascular lumen was almost two-fold higher than for unlabeled pQDs (Figure 3.2D). 


\section{$S_{0}$-effect}

For both cNGR-pQDs and unlabeled pQDs, changes in the scaling factor $S_{0}$ colocalized strongly with $\Delta R_{1}$ (Figure $3.2 \mathrm{~B}$ ). The $S_{0}$-effect is likely caused by field inhomogeneities ( $T_{2}^{*}$ effect) in the vicinity of the contrast agent, induced by the magnetic properties of QDs [159] and the dense gadolinium concentration on the particle. Analogous to iron oxide particles, such properties result in locally reduced transverse relaxation times $T_{2}$ and $T_{2}^{*}$, a shift in local resonance frequency, and a broader water resonance line, which is reflected by a decrease in $S_{0}$, i.e. a reduced proton visibility $[154,160]$. Therefore, $\Delta R_{1}$ and $\Delta S_{0}$ both represent contrast agent presence.

\section{Spatial heterogeneity}

To explore the absolute differences between tumor rim, tumor core, and muscle, $Q C_{R_{1}}$ and $Q C_{S_{0}}$ were determined for each tissue type for $c N G R-p Q D s$ and unlabeled pQDs (Figure 3.3 A/B). Administration of cNGR-pQDs resulted in an approximately 50-fold increase in $Q C_{R_{1}}$ in the angiogenic rim compared with tumor core or muscle tissue. For unlabeled pQDs, significant differences were also found between tumor rim and core, and tumor rim and muscle tissue, although the net increase in $Q C_{R_{1}}$ was lower than for cNGR-pQDs. The decreases in $S_{0}$ showed the same trend as the increases in $R_{1}$ (cf. Figure $3.3 \mathrm{~A} / \mathrm{B}$ ).

For each of the three tissue types, no significant differences in $Q C_{R_{1}}$ or $Q C_{S_{0}}$ were found between cNGR-pQDs and unlabeled pQDs. Since $\Delta R_{1}$ and $\Delta S_{0}$ were shown to accurately colocalize (Figure 3.2), $Q C_{R_{1}}$ data were combined with $Q C_{S_{0}}$ to a summary measure as described in Section 3.2. This resulted in a statistically significant difference between cNGR-pQDs and unlabeled pQDs for the tumor rim only (Figure $3.3 \mathrm{~A} / \mathrm{B}$ ).

Figure 3.2 (facing page): $T_{2}$-weighted anatomical images with color overlay of $\Delta R_{1}(\mathrm{~A})$ and $\triangle S_{0}(\mathrm{~B})$ for tumor $(\mathrm{T})$ and muscle $(\mathrm{M})$ tissue of mice injected with cNGR-pQDs or unlabeled pQDs ( $\mathrm{n}=7$ for both groups). Changes in $R_{1}$ were most pronounced at the tumor rim for cNGR-pQDs. Although an $R_{1}$ increase in the tumor rim was also observed for unlabeled pQDs, the average response was 3-fold lower when compared with cNGR-pQDs, indicating a high specificity of cNGR for angiogenic tumor endothelium. This is further supported by the low changes in $R_{1}$ found in muscle tissue. Changes in $S_{0}(\mathrm{~B})$ colocalized almost completely with changes in $R_{1}(\mathrm{~A})$. Representative TPLSM images of tumor (C) and muscle tissue (D) showing pQD signal in red and endothelial cell specific $\alpha$ CD31-FITC in green. cNGR-pQDs accurately colocalized with tumor endothelial cells, indicating binding of the contrast agent to the tumor endothelium (C). cNGR-pQDs were also detected in muscle tissue with TPLSM (arrows in Figure D), although to a much lesser extent than in tumor tissue. cNGR-pQDs did not display any colocalization with muscle endothelial cells and were only found intraluminally. Unlabeled pQDs were not or only sparsely detected in both tumor and muscle tissue. Bar: $50 \mu \mathrm{m}$. 


\subsection{RESULTS}
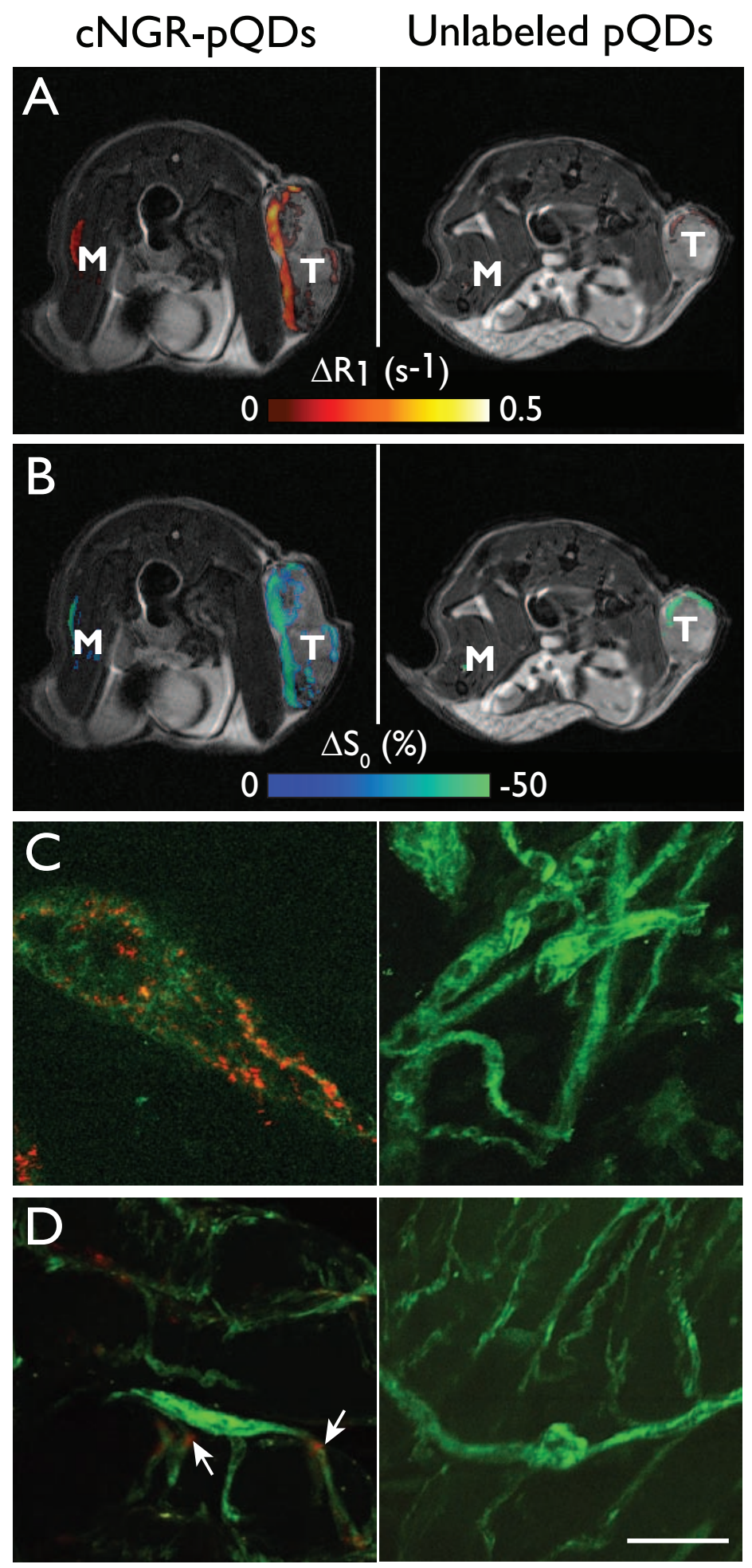

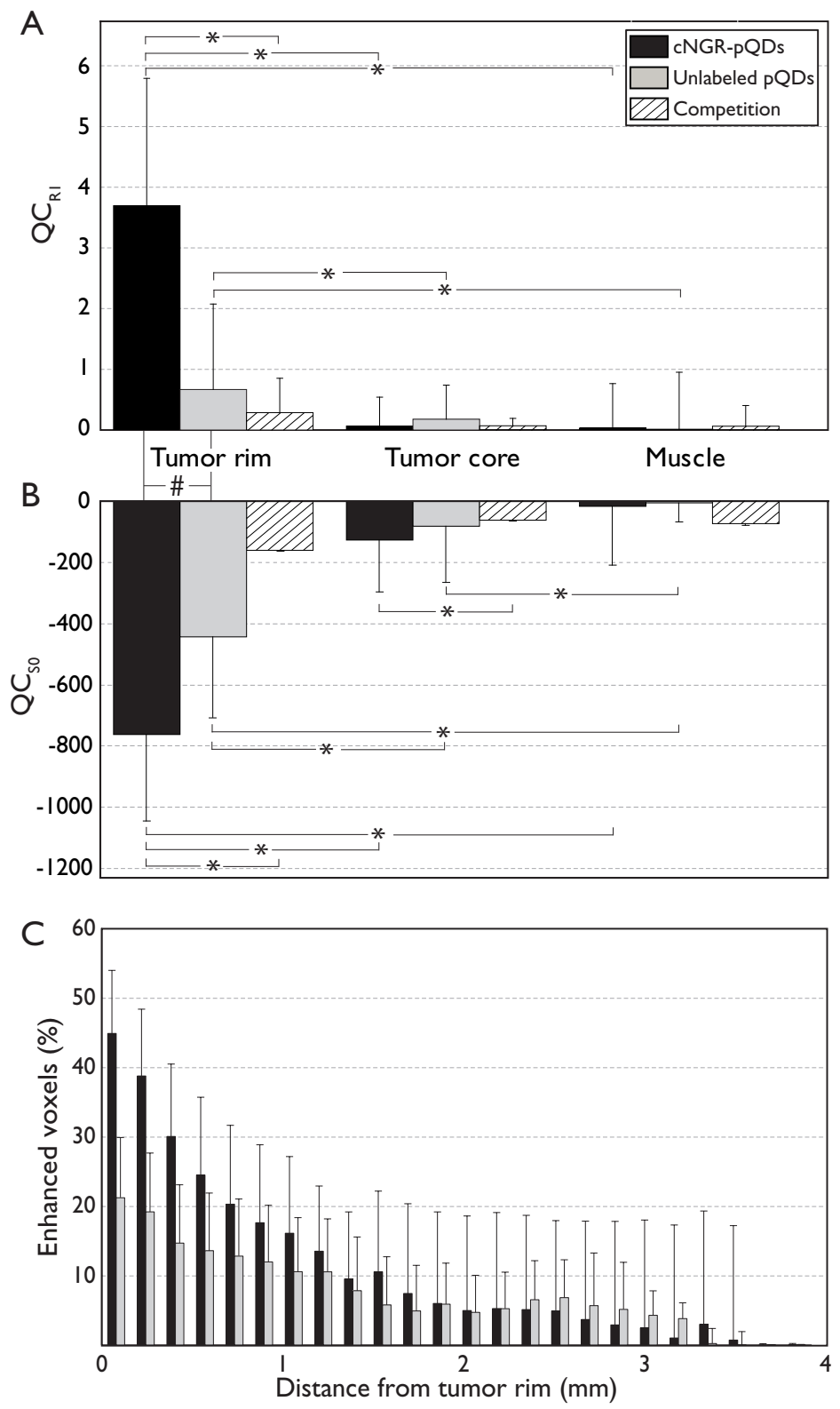

Figure 3.3: Spatial distribution of angiogenic activity. A/B) Quantitative contrast as derived from changes in $R_{1}\left(Q C_{R_{1}}, \mathrm{~A}\right)$ and from changes in $S_{0}\left(Q C_{S_{0}}, \mathrm{~B}\right)$ for tumor rim, tumor core and hindlimb muscle tissue. Data are shown for cNGR-pQDs $(n=7)$, unlabeled pQDs $(n=7)$, and the competition experiment of cNGR-pQDs with excess unconjugated cNGR $(n=4)$. C) Percentage of enhanced voxels at a certain distance versus distance from the tumor rim for cNGR-pQDs and unlabeled pQDs. Although enhanced voxels were mostly found at the tumor rim for both contrast agents, more than twice as many rim voxels were enhanced for cNGR-pQDs than for unlabeled pQDs. In the tumor core, similar values were found for both contrast agents. Values are presented as median \pm standard error. ${ }^{*} \mathrm{P}<0.05$. $\# \mathrm{P}<0.05$ for the O'Brien-Läuter summary measure of $Q C_{R_{1}}$ and $Q C_{S_{0}}$ (see text). 
To further investigate the spatial distribution of angiogenic activity in the tumor, the percentage of significantly enhanced voxels was calculated as a function of the distance to the tumor rim (Figure 3.3C). Although the highest signal increase was found at the tumor rim for both contrast agents, more than twice as many rim voxels were enhanced for cNGR-pQDs than for unlabeled pQDs. In the tumor core, similar enhancements were found for both contrast agents. These findings qualitatively concur with previous findings, showing that angiogenic activity is most pronounced at the tumor rim for this tumor model $[113,158]$.

Subsequently, half-value-depths were calculated for both cNGR-pQDs and unlabeled $\mathrm{pQDs}$. High values indicate a more homogeneous distribution of enhanced voxels over the entire tumor and thus a low spatial heterogeneity, whereas low values indicate a high spatial variation. For cNGR-pQDs and unlabeled pQDs, the half-value-depths were 0.6 and $1.1 \mathrm{~mm}$, respectively, indicating a stronger contrast between tumor rim and core for cNGR-pQDs, which suggests that cNGR-pQDs allow a better differentiation between tumor rim and core than unlabeled pQDs.

\section{Competition experiment}

Intravenous injection of a 1000-fold excess of unconjugated CNGR 10 minutes after administration of cNGR-pQDs resulted in a statistically significant decrease in $Q C_{R_{1}}$ and $\mathrm{QC}_{S_{0}}$ for the tumor rim (Figure $3.3 \mathrm{~A} / \mathrm{B}$ ). With TPLSM, cNGR-pQDs were barely detected in the tumor rim, which confirmed the MRI results (data not shown). These results therefore indicate that binding of cNGR-pQDs to tumor ECs is specific, reversible, and can be competed with unconjugated cNGR.

\section{Biodistribution}

Figure 3.4 shows the relative MRI signal intensities for the blood and major organs recorded approximately one hour after the administration of cNGR-pQDs, unlabeled pQDs, or no contrast agent. No differences were found between cNGR-pQDs and unlabeled pQDs. Both contrast agents accumulated mainly in the spleen, liver, and kidneys (Figure 3.4), which was confirmed by TPLSM and corresponds to previous findings [161]. Due to the intravenous administration, pQDs were also expected to accumulate in the lung. However, MRI has only limited signal sensitivity in the lung due to the inherently low signal intensity and air-tissue interfaces. With TPLSM, pQDs could be clearly detected in the lung (Figure 3.4), although microscopic imaging was hampered by tissue movement caused by heating of the sample during excitation, resulting in expansion of air in the pulmonary alveoli.

\section{Contrast agent relaxivity}

The ionic $T_{1}$ relaxivity of cNGR-pQDs, i.e. per Gd ion, was $7.1 \pm 0.4 \mathrm{mM}^{-1} \mathrm{~s}^{-1}$ at $7 \mathrm{~T}$ and $20^{\circ} \mathrm{C}$, which lies in the expected range for macromolecular contrast agents and is in correspondence with previously reported values for Annexin A5 conjugated pQDs [162]. 


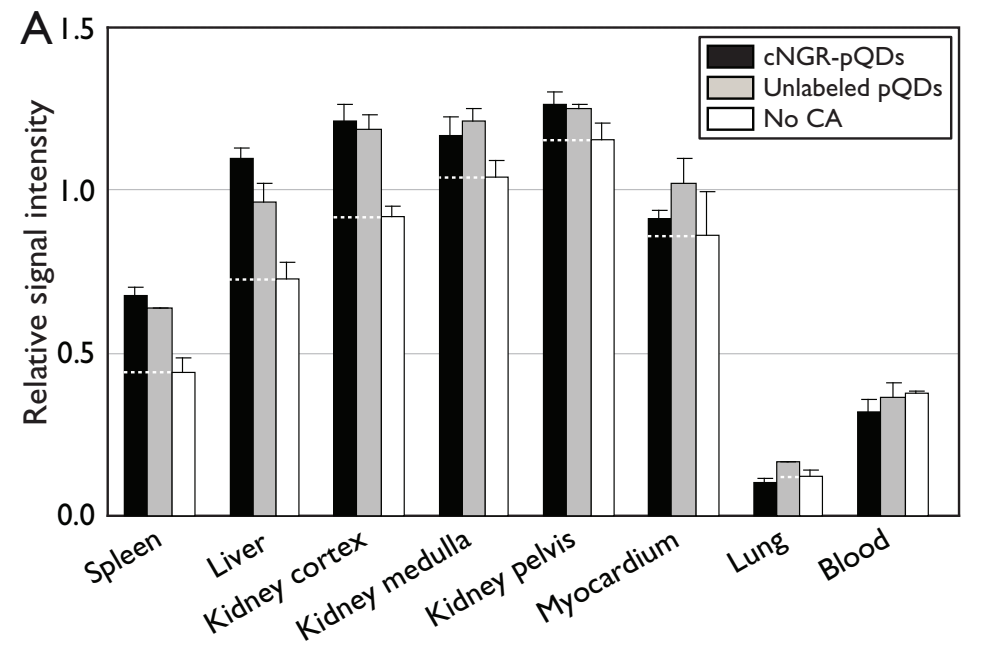

B

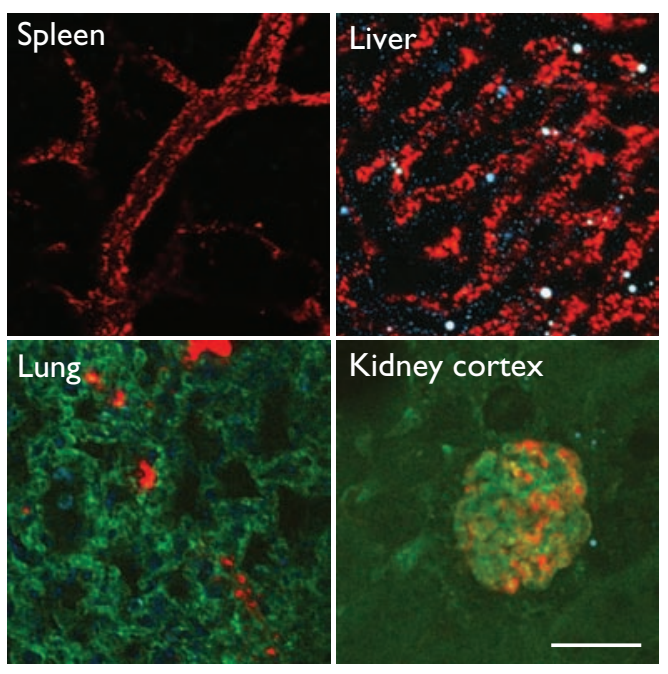

Figure 3.4: Biodistribution of cNGR-pQDs and unlabeled pQDs ( $n=2$ for each group). A) Postmortem $T_{1}$-weighted $\mathrm{MRI}$ results. Signal intensities were normalized to hindlimb muscle signal and subsequently averaged. No CA: no contrast agent administration $(n=2)$. Both cNGR-pQDs and unlabeled pQDs were found to accumulate mainly in the spleen, liver, and kidneys. Values are represented as median \pm standard error. B) Representative TPLSM images of spleen, liver, lung, and kidney. Since a similar biodistribution was found for cNGRpQDs and unlabeled pQDs, no differentiation was made for the TPLSM results. Red: pQDs, Green: $\alpha$ CD31-FITC, Blue: autofluorescence, Bar: $50 \mu \mathrm{m}$. 


\subsection{Discussion}

\section{Current findings}

In this study, the ability of cNGR-labeled paramagnetic QDs to visualize and quantify angiogenic activity in LS174T tumors was evaluated using two complementary imaging modalities: in vivo MRI and ex vivo TPLSM. To our knowledge, this study shows the first results of the application of cNGR for molecular MRI. First, cNGRpQDs were found to have a 3-fold higher quantitative MRI contrast in the tumor rim, i.e. the tumor region with the highest angiogenic activity, compared with unlabeled pQDs. Second, cNGR-pQDs were barely detectable in muscle tissue, indicating a high specificity of cNGR-pQDs for angiogenic vessels. Third, ex vivo TPLSM showed colocalization of cNGR-pQDs, but not unlabeled pQDs, with ECs in the tumor vasculature. Fourth, cNGR-pQDs allowed a more accurate assessment of the rim-core heterogeneity in tumor angiogenic activity. Fifth, the competition experiment indicated that the binding of cNGR-pQDs to tumor ECs is specific, reversible, and can be competed with excess unconjugated cNGR. Finally, no qualitative differences in biodistribution were found between cNGR-pQDs and unlabeled pQDs with MRI and TPLSM. For a full quantitative biodistribution assessment, positron emission tomography would be better suited since the local relaxivity of the MRI contrast agent, which is required to convert $R_{1}$ to concentrations, is unknown. Taken together, the presented results designate cNGR as an effective ligand for discriminating between quiescent and activated endothelium, and for quantifying the extent of tumor angiogenic activity.

In correspondence with other studies $[113,158]$, our results indicate that angiogenic activity is highest at the tumor rim, which was reflected by a high $Q C_{R_{1}}$ and a strongly negative $Q C_{S_{0}}$ for cNGR-pQDs. This was supported by the statistically significant difference between cNGR-pQDs and unlabeled pQDs found using the summary value. Unlabeled $\mathrm{pQDs}$ also showed differences between tumor rim versus core and muscle, albeit smaller than for the cNGR-pQDs. This is likely due to the tumor rim's intrinsically higher vascular density and corresponding blood pool fraction, resulting in a larger amount of circulating contrast agent compared with tumor core or muscle tissue [158]. Additionally, heterogeneous blood flow and long wash-in and wash-out times of macromolecular contrast agents, which was previously described for dendritic agents [126], may have contributed to a prolonged retention of unlabeled $\mathrm{pQDs}$ in the tumor peripheral vasculature.

\section{Methodological considerations}

Non-linear fitting of the IR-signal over a range of inversion times provided a sensitive and reliable method for detecting $R_{1}$ changes induced by contrast agent binding. Compared to signal intensity measurements, it is relatively independent of technical 
settings, e.g. repetition time, echo time, and flip angle, thereby allowing objective comparison between different subjects, both spatially and temporally. A disadvantage of the quantitative approach is the lengthy acquisition time since an adequate number of data points is required for accurate fitting of the IR curve. Although prolonged preand post-contrast acquisition of a single image at a fixed inversion time may also allow accurate detection of changes in signal intensity, this will not provide quantitative information on $R_{1}$ and $S_{0}$. In our quantitative approach, coregistration of these parameters ensured increased sensitivity to detect differences between cNGR-pQDs and unlabeled $\mathrm{pQDs}$ and is therefore preferred over acquisition of a single image.

Theoretically, the measured longitudinal relaxation rates and contrast agent relaxivity allow estimation of local contrast agent concentration and might be used to gain insight in the density of the molecular target. However, the conditions under which the relaxivity was determined differ strongly from the in vivo situation. Not only is the relaxivity affected by the particle's chemical environment, i.e. aqueous buffer versus blood plasma [16], specific binding to vascular endothelium reduces its tumbling rate, thereby increasing the relaxivity. Taken together, this would lead to an overestimation of the local concentration in vivo. Unfortunately, accurate measurements of in vivo relaxivity are currently unavailable.

\section{Contrast agent}

Quantum dots were chosen as scaffold to enable bi-modal, i.e. MRI and TPLSM, visualization of tumor angiogenic activity, which is an essential step in the characterization and validation of cNGR as a targeting ligand. Furthermore, streptavidin-coated QDs provide a suitable and versatile research scaffold to identify and test other potential targeting ligands. In addition, QDs show minimal extravasation, both from healthy and hyperpermeable tumor vasculature (unpublished observations), which is beneficial for EC targeting. However, QDs may pose serious health limitations due to the potential release of toxic cadmium ions. Although this can be prevented by effective shielding of the core $[163,164]$, QDs are not cleared from the body and accumulate in spleen, liver, and kidneys. Cadmium-based QDs will therefore not be approved for clinical application. Recently developed non-toxic and renally excretable QDs may provide a potential solution for this problem $[165,166]$. Nevertheless, once a robust MRI method has been accepted in clinical practice, validation with luminescent particles is no longer necessary and clinically more suitable particles may be applied.

The magnetic and semi-conductive properties of QDs give rise to field inhomogeneities when placed inside a magnetic field [159], which likely result in a local decrease of the transverse relaxation times $T_{2}$ and $T_{2}^{*}$. In addition, $T_{2}$ contrast becomes more effective at high field strength, whereas $T_{1}$ contrast decreases. Using a standard multi-slice multi-echo spin echo sequence, an average $R_{2}$ increase of $5.7 \mathrm{~s}^{-1}$ upon cNGR-pQD injection was detected in the tumor rim at a mean tumor 
pre-contrast $R_{2}$ of approximately $27 \mathrm{~s}^{-1}$. However, the percentage of significantly enhanced voxels in the tumor rim was only $4 \%$, which is considerably lower than the $42 \%$ found for $R_{1}$. This demonstrates that $T_{2}$ changes did not interfere with the effects on $T_{1}$ and $S_{0}$. Consequently, the $T_{1}$ and $S_{0}$ quantification was more sensitive in discriminating between angiogenic activity in the tumor rim, tumor core, and muscle tissue than $T_{2}$ (see also Chapter 4).

\section{Clinical perspectives}

Regarding the potential clinical applicability, quantitative molecular MRI with a suitable contrast agent has a number of advantages over the currently used immunohistochemical methods to quantify tumor angiogenic activity. First, molecular MRI is non-invasive and does not interfere with tissue integrity. Second, it can probe the entire tumor, whereas immunohistochemistry requires biopsies at one or multiple selected locations. Third, it allows covisualization of angiogenic activity with local anatomy. Fourth, tumor status or therapeutic response may be objectively monitored over time due to the absolute quantification methodology. Finally, molecular MRI allows direct detection of activated endothelium in functional vasculature, while immunohistochemistry measures both perfused and non-perfused vessels.

With respect to the applied tumor model, a human colorectal adenocarcinoma, MRI is clinically important for local T-staging of rectal cancer and for the identification of tumors close to or invading the mesorectal fascia [167]. On diagnostic $T_{2}$-weighted images, however, it remains difficult to differentiate between fibrotic tissue and viable tumor cells. Molecular MRI of angiogenesis may facilitate this demarcation, since only viable tumor cells induce angiogenesis, which may be visualized upon administration of the targeted contrast agent.

Besides the availability of suitable contrast agents, clinical implementation of quantitative molecular MRI requires rapid imaging techniques. Possible sequences that allow fast quantification of relaxation times are Look-Locker [168], IR-trueFISP [169], and the recently described QRAPTEST [170]. However, these methods are relatively sensitive to subject movement and field inhomogeneities, although the Look-Locker method was recently modified to allow in vivo $T_{1}$-mapping of the heart [171]. Thus, the development of fast quantification of relaxation rates seems to support future clinical application of quantitative molecular MRI.

In summary, we have shown that cNGR-labeled paramagnetic quantum dots are suitable for the non-invasive visualization and quantification of tumor angiogenic activity using in vivo molecular MRI. These results provide a promising basis for further developments in contrast agent design and synthesis, data acquisition, and post-processing techniques, which may be valuable for future clinical applications to pathologies in which abnormal vessel growth plays a pivotal role. 



\section{4}

\section{Gadolinium-labeled quantum dots for molecular MRI: $R_{1}$ versus $R_{2}$ mapping}

Quantum dots labeled with paramagnetic Gd-chelates (pQDs) can be applied as contrast agent for preclinical molecular MRI combined with fluorescence microscopy. Besides increasing the $R_{1}$ relaxation rate, $\mathrm{QQDs}$ may increase the $R_{2}$ relaxation rate, which might be related to their magnetic properties. Furthermore, molecular MRI experiments are primarily conducted at high magnetic fields, where $R_{1}$ relaxation becomes less effective, and the use of $R_{2}$ relaxation as a source of contrast may become attractive. Consequently, the optimal method of contrast enhancement using $\mathrm{pQDs}$ is a priori unknown. The objective of this study was to compare $R_{1^{-}}$ and $R_{2}$-based contrast enhancement, proton visibility, and changes thereof induced by $\mathrm{pQDs}$ targeted to the angiogenic vasculature of murine tumors, using in vivo $R_{1}$ and $R_{2}$ mapping. At a field strength of 7 Tesla, $R_{1}$-based measures were superior to $R_{2}$-based measures in detecting both the level and spatial extent of contrast agent induced relaxation rate changes.

Magnetic Resonance in Medicine. 2009, provisionally accepted 


\subsection{Introduction}

Molecular MRI is defined as the in vivo characterization and measurement of biological processes at a cellular or molecular level using MRI techniques [172]. Typically, contrast agents are used which home to biomarkers that are upregulated during disease. This is achieved via high-affinity ligands (e.g. peptides, antibodies) conjugated to the contrast agent. To increase the low sensitivity of molecular MRI, large particles with slow tumbling rates carrying a high payload of gadolinium are used to increase contrast agent relaxivity [109]. Furthermore, multiple ligands are attached to the contrast agent to enhance target affinity $[19,109]$.

Despite the submillimeter resolution of MRI, differentiation between contrast agent bound to the biological target and unbound intravascular contrast agent is problematic. Moreover, signal from the blood pool would overshadow the signal from bound contrast agent, thereby complicating image interpretation. Validation of the MRI results with a subcellular resolution technique, such as fluorescence microscopy, is therefore essential for the development and characterization of molecular MRI contrast agents. To this end, experimental contrast agents are often bimodal, e.g. paramagnetic and fluorescent, which allows in vivo MRI and subsequent validation with ex vivo fluorescence microscopy [173].

Quantum dots (QDs) are highly luminescent semiconductor nanoparticles that can be used as contrast agent scaffolds for molecular MRI. The rationale for using QDs in experimental MRI studies is three-fold. First, QDs have highly favorable photophysical properties, including a broad excitation spectrum, a small size-dependent emission spectrum and limited photo-bleaching $[145,146]$. Especially for the in vivo or in situ environment, QDs are at least 20 times brighter than organic dyes $[174,175]$. This allows validation of the molecular MRI results with independent fluorescence microscopy techniques (Chapter 3). Second, multiple targeting ligands can be attached to the QD surface, which increases the binding efficacy of the contrast agent through multivalent interactions [112]. Third, QDs can be labeled with multiple gadolinium constructs, for instance via incorporation into a lipid coating [176-178] or via biotinstreptavidin interactions $[119,162]$, thereby amplifying the MRI signal.

Administration of QDs labeled with paramagnetic Gd-chelates (pQDs) generates an increase in the local longitudinal relaxation rate $R_{1}\left(=1 / T_{1}\right)$ due to the interaction of water protons with surface-bound gadolinium. Furthermore, phantom experiments showed that QDs generate an increase in the local transverse relaxation rate $R_{2}$ $\left(=1 / T_{2}\right.$, see Appendix $\left.B\right)$. It is hypothesized that this is related to their magnetic properties [159]. In addition, the Gd-chelates also contribute to the $R_{2}$ relaxation. The net contrast enhancement induced by $\mathrm{PQDs}$ therefore likely involves $R_{1}$ - as well as $R_{2}$-effects. It is a priori unknown whether $R_{1^{-}}$or $R_{2}$-based methods will show the strongest contrast enhancement upon administration of pQDs and hence, which method allows the most accurate assessment of $\mathrm{pQD}$ induced changes in image contrast. 


\subsection{INTRODUCTION}

Table 4.1: Intrinsic in vivo tissue $R_{1}$ and $R_{2}$ relaxation rates measured at 7 Tesla. Values are represented as mean $\pm S D$, where $S D$ is the regional standard deviation over the tissue. Available literature values, measured in mice and rats at $7 \mathrm{~T}$, are presented as well.

\begin{tabular}{|c|c|c|c|c|}
\hline \multirow[b]{3}{*}{ Liver } & \multicolumn{2}{|c|}{$R_{1}\left(\mathrm{~s}^{-1}\right)$} & \multicolumn{2}{|c|}{$R_{2}\left(\mathrm{~s}^{-1}\right)$} \\
\hline & Present data & Literature $^{a}$ & Present data & Literature $^{a}$ \\
\hline & $1.34 \pm 0.14$ & $1.13 \pm 0.25[179]^{b}$ & $45.6 \pm 5.8$ & $N A^{c}$ \\
\hline Spleen & $0.91 \pm 0.05$ & $0.77 \pm 0.15[179]^{b}$ & $29.9 \pm 3.1$ & NA \\
\hline Kidney cortex & $1.19 \pm 0.06$ & $0.97 \pm 0.13[181]^{d}$ & $27.8 \pm 4.1$ & $55.6 \pm 6.2[181]^{d}$ \\
\hline Kidney medulla & $0.86 \pm 0.04$ & $0.90 \pm 0.09[181]^{d}$ & $23.3 \pm 3.6$ & $43.5 \pm 3.8[181]^{d}$ \\
\hline Skeletal muscle & $1.00 \pm 0.05$ & $\approx 0.7$ & $44.7 \pm 2.7$ & $43.9 \pm 2.1$ [183] \\
\hline Brain & $0.96 \pm 0.05$ & $0.63 \pm 0.02[184]$ & $22.5 \pm 1.8$ & $27.8 \pm 1.5[184]$ \\
\hline Blood & $0.65 \pm 0.01$ & $0.56 \pm 0.00 \quad[182]$ & $38.4 \pm 10.4$ & NA \\
\hline
\end{tabular}

a Values were calculated from $T_{1}$ and $T_{2}$ literature values. ${ }^{b}$ Extrapolated from data obtained at $1-100 \mathrm{MHz}(0.02-2.4 \mathrm{~T})$. ${ }^{\mathrm{c}}$ Not available. ${ }^{\mathrm{d}}$ Postmortem results.

On the one hand, it is expected that a $T_{1}$-weighted inversion recovery has a higher sensitivity to detect changes in relaxation rate compared to a $T_{2}$-weighted spin echo technique (see Appendix C). Furthermore, the relative change in relaxation rate is related to the change in contrast agent concentration, and the ratio of relaxivity and intrinsic relaxation rate. As the ionic longitudinal and transverse relaxivities are generally comparable for most paramagnetic contrast agents [18], the relaxation rate change depends mostly on the intrinsic tissue relaxation rate. Considering that the intrinsic tissue $R_{2}$ values are significantly higher than $R_{1}$ values (Table 4.1, [179]), contrast induced changes in $R_{2}$ are likely more difficult to detect than changes in $R_{1}$. This is further supported by the wider absolute range in $R_{2}$ than in $R_{1}(0.7$ versus $23.1 \mathrm{~s}^{-1}$, respectively, Table 4.1), which was also found in humans at lower field strengths of 1.5 and 3 Tesla [16]. On the other hand, molecular MRI experiments are primarily conducted at dedicated animal systems operating at field strengths of approximately 4 Tesla or higher. This leads to a decreased $T_{1}$ contrast enhancement, since both the intrinsic tissue $R_{1}$ and the contrast agent's longitudinal relaxivity $r_{1}$ decrease with increasing magnetic field $[18,134,180]$. In contrast, $R_{2}$ and the transverse relaxivity $r_{2}$ are less field strength dependent, suggesting that $T_{2}$ contrast enhancement may become more important at high field strengths.

Here, we describe an MRI method to evaluate tissue $R_{1}$ and $R_{2}$ relaxation rates, proton visibility $\left(S_{0}\right)$, and changes thereof induced by $\mathrm{pQDs}$ targeted to the angiogenic vasculature of murine tumors at 7 Tesla. Angiogenesis is a well-suited and wellstudied process for molecular MRI since the biological targets are easily accessible for intravenous contrast agents. pQDs were labeled with the cyclic tripeptide asparagineglycine-arginine (cNGR), which was shown to home to CD13, an aminopeptidase that is upregulated on endothelial cells of angiogenic tumor vessels [136, 140,141]. Previous work showed that administration of cNGR-pQDs resulted in an increase in $R_{1}$ in the angiogenic tumor periphery, whereas injection of control pQDs without cNGR 
did not (Chapter 3). These findings were validated using two-photon laser scanning microscopy, which showed that cNGR-pQDs accurately colocalized with vascular endothelial cells in the tumor rim.

\subsection{Methods}

\section{Contrast agent preparation}

cNGR-labeled pQDs were prepared in Section 3.2. Briefly, streptavidin-coated CdSebased QDs (1 $\mu \mathrm{M}$ solution in borate buffer $\mathrm{pH}$ 8.3, $585 \mathrm{~nm}$ emission) were purchased from Invitrogen (Breda, The Netherlands). Contrast agent was prepared freshly for each experiment by mixing $100 \mu \mathrm{L}$ of QDs with biotinylated poly(lysine) dendritic wedge, a structure comprising 8 Gd-DTPA groups, and biotinylated cNGR in a molar ratio of 1:24:6. Both biotin-poly(lysine) dendritic wedge and biotin-cNGR were synthesized via tBoc solid-phase peptide synthesis, as described previously [147-149]. Each cNGR-pQD carried a maximum of 192 Gd ions and 6 cNGR ligands (Chapter 3). The particle's hydrodynamic diameter was measured using dynamic light scatting at $25{ }^{\circ} \mathrm{C}$ on a Malvern zetasizer nano-S (Malvern Instruments Inc, Worcestershire, UK).

\section{Animal model}

All experiments were approved by the institutional ethics committee on the care for experimental animals. Tumors were induced in 15 week old male Swiss nu/nu mice via subcutaneous injection of $1.5-3 \times 10^{6}$ human colorectal adenocarcinoma cells (LS174T, American Type Culture Collection CL-188, Rockville, MD) in the flank. Tumors grew for approximately 14 days and had a size of approximately $1.0 \mathrm{~cm}^{3}$ at the day of MRI. In total, seven mice were included.

Mice were anesthetized prior to the MRI examination using $1.5-2.0 \%$ isoflurane (Abbott Laboratories Ltd, Queensborough, UK) in medical air and were placed prone in an animal holder with built-in anesthesia mask. Normothermic conditions were maintained using a warm water pad. Respiration rate and body temperature were monitored with an MR compatible small animal monitoring system (SA Instruments, Stony Brook, NY). Contrast agent ( $120 \mu \mathrm{L}, 1 \mu \mathrm{M}$ cNGR-pQDs) was injected during the MRI experiment via the jugular vein.

\section{MRI protocol}

All experiments were performed on a Bruker Biospec 70/30 USR MRI system (Bruker Biospin, GmbH, Ettlingen, Germany), interfaced to an AVANCE II console. The BGA12-S mini imaging gradient (maximum gradient strength $720 \mathrm{mTm}^{-1}$, slew rate $6000 \mathrm{Tm}^{-1} \mathrm{~s}^{-1}$ ) and a $3.5 \mathrm{~cm}$ inner diameter quadrature volume resonator were used. 


\section{Relaxivity}

cNGR-pQDs were repeatedly diluted by a factor two in $1 \mu \mathrm{M}$ borate buffer $\mathrm{pH}$ 8.3. In total, 6 samples were prepared with gadolinium concentrations ranging from 0.003 to $0.13 \mathrm{mM}$. Exact concentrations were determined using Inductively Coupled Plasma Mass Spectrometry (MiPlaza Materials Analysis, Eindhoven, The Netherlands). $R_{1^{-}}$ values were determined using a series of inversion recovery (IR) measurements with increasing inversion times (TI) of 50, 100, 250, 500, 800, 1000, 1500, 2000, 2500 and $3000 \mathrm{~ms}$. Repetition time (TR) and echo time (TE) were 8000 and $8.2 \mathrm{~ms}$, respectively. $R_{2}$-values were measured using a multi-slice multi-echo (MSME) spin echo sequence with a TR of $8000 \mathrm{~ms}$ and TE ranging from 10 to $80 \mathrm{~ms}$ with an echo interval of $10 \mathrm{~ms}$. The longitudinal relaxivity $r_{1}$ and transverse relaxivity $r_{2}$ were given by the slope of a linear fit of $R_{1}$ and $R_{2}$ versus gadolinium concentration, respectively.

\section{Relaxometry}

Intrinsic tissue $R_{1}$ and $R_{2}$ were determined in vivo for the liver, spleen, kidney, skeletal muscle, and brain of a healthy mouse using IR (TR 3000 ms, TE 8.2 ms, TI 500, 750, 1000, 1500, 2000, and $2500 \mathrm{~ms}, 4$ signal averages) and MSME (TR 2500, TE 10, $20 \ldots 80 \mathrm{~ms}, 4$ signal averages) sequences, respectively. Subsequently, the mouse was sacrificed and the blood was collected via a heart puncture for $R_{1}$ and $R_{2}$ measurement. Clotting was prevented using heparin. Scan parameters were equal to the in vivo relaxometry experiments, except that TR was 10 seconds for both the IR and MSME and that TI was 380, 500, 750, 1000, 1500, 2000, 3000, 5000, and $8000 \mathrm{~ms}$ for the IR images.

\section{Molecular MRI}

Tumors were localized using $T_{2}$-weighted spin echo images (TR 4200, TE $37.5 \mathrm{~ms}$ ). Pre- and post-contrast $R_{1}$-values were determined using a series of IR images (TR 4000, TE 8.2, TI 500, 1000, 1500, 2000, 2500, and $3500 \mathrm{~ms}$, acquisition matrix $192 \times 192$ interpolated via zero-filling to $256 \times 256$, slice thickness $1.2 \mathrm{~mm}$, spatial resolution $0.16 \times 0.16 \times 1.2 \mathrm{~mm}^{3}$, total scan time 18 minutes). Pre- and postcontrast $R_{2}$-values were measured using an MSME experiment (TR 4000, TE 10, 2 . . .80 ms, acquisition matrix $128 \times 128$, slice thickness $1.2 \mathrm{~mm}$, spatial resolution $0.32 \times 0.32 \times 1.2 \mathrm{~mm}^{3}$, total scan time 6.5 minutes). On average, 15 slices were recorded (range 11-22, depending on tumor size and orientation). Post-contrast measurements were performed approximately 30 minutes after injection of cNGRpQDs. For a plasma half life of approximately 7 minutes (Chapter 5), this results in a systemic contrast agent concentration of $5 \%$ of the maximum value. Image acquisition at 30 minutes post contrast administration therefore ensures adequate time for contrast agent circulation and target binding, while simultaneously providing a low background signal of unbound intravascular agent. 


\section{Data analysis}

Images were spatially coregistered using MIRIT [151] to correct for possible animal motion within the IR or MSME series. Next, images were smoothed with a threedimensional Gaussian kernel with a full-width-at-half-maximum of 2.5 times the voxel size. Regions of interest defining tumor and muscle tissue were drawn manually using MRIcro [152]. Both $T_{1^{-}}$and $T_{2}$-weighted images were used to correctly delineate the tumor from surrounding tissue.

All further data processing was performed in Matlab (The Mathworks, Natick, MA). Pre- and post-contrast $R_{1}$ and $R_{2}$ were determined on a voxel-by-voxel basis by non-linear curve fitting of the corresponding signal intensity functions (see Appendix C) using the Levenberg-Marquardt optimization algorithm. The quality of the $R_{1}$ and $R_{2}$ fits was determined using the fit error, which was defined as:

$$
\text { fit error }=100 \% \times \sqrt{\frac{\sum_{i}\left(S_{\text {fit }}^{i}-S_{\text {exp }}^{i}\right)^{2}}{\sum_{i}\left(S_{\text {fit }}^{i}\right)^{2}}}
$$

Here, $S_{\exp }^{i}$ is the experimentally determined signal as a function of discrete values of $\mathrm{TI}^{i}$ or $\mathrm{TE}^{i}$ for IR and MSME experiments, respectively, and $S_{\text {fit }}^{i}$ is the calculated signal based on the results of the non-linear fitting. The relative tissue contrast between tumor and muscle tissue was defined as:

$$
\left|\frac{\tilde{R}_{1,2}^{\text {muscle }}-\tilde{R}_{1,2}^{\text {tumor }}}{\tilde{R}_{1,2}^{\text {tumor }}}\right| \times 100 \%
$$

where $\tilde{R}_{1,2}$ is the median relaxation rate value. Changes in relaxation rate were defined as the difference between post- and pre-contrast values $\left(\Delta R_{1,2}=R_{1,2}^{\text {post }}\right.$ $\left.R_{1,2}^{\text {pre }}\right)$.

Detection limits for $\Delta R_{1,2}$ were determined using Monte Carlo simulations. Theoretical signal intensity curves for IR and MSME were generated using in vivo determined pre- and post-contrast relaxation rates, scaling factors $\left(S_{0}\right.$, see Appendix C), and noise levels. Experimental noise levels were measured in a region outside the mouse. Next, 10.000 Monte Carlo runs were performed to obtain a mean fitted relaxation rate. The corresponding standard deviation multiplied by a factor 2 served as the detection limit for $\Delta R_{1,2} . \Delta R_{1}$ and $\Delta R_{2}$ were considered significant when larger than $0.01 \mathrm{~s}^{-1}$ and $5.0 \mathrm{~s}^{-1}$, respectively.

As a summary measure of both the level and spatial extent of contrast enhancement, the quantitative contrasts from $R_{1}$ and $R_{2}$ were defined as the product of the average change in relaxation rate and the percentage of significantly enhanced voxels $\left(\mathrm{QC}_{R_{1}}=R_{1} \cdot P_{\mathrm{vox} R_{1}}\right.$ and $\left.\mathrm{Q} C_{R_{2}}=R_{2} \cdot P_{\mathrm{vox} R_{2}}\right)$. 


\section{Tumor rim/core}

The tumor rim was defined as an approximately $1 \mathrm{~mm}$ thick peripheral zone, as described previously (Chapter 3, [81, 155]). Care was taken to include approximately the same regions for the IR and MSME images. On average, the rim comprised 30\% of the total tumor volume. The tumor core was defined as the difference between whole tumor and tumor rim regions. A single voxel contour was used to calculate the distance to the tumor rim for each voxel.

\section{Statistical analysis}

Statistical analyses were performed in SPSS 14.0 (SPSS, Chicago, III). Differences between paired samples were considered statistically significant when $P<0.05$ according to a non-parametric Wilcoxon signed ranks test.

\subsection{Results}

\section{Contrast agent characterization}

QDs have a hydrodynamic diameter of $23.4 \mathrm{~nm}$, with a polydispersity index of 0.18 , as measured using dynamic light scattering. Upon addition of the gadolinium construct, the hydrodynamic radius increased to $28.9 \mathrm{~nm}$, with a polydispersity index of 0.20 . The difference of $5.5 \mathrm{~nm}$ between QDs and pQDs corresponds with the size of the poly(lysine) dendritic gadolinium-wedge of $3 \times 5 \mathrm{~nm}^{2}$, as estimated through de novo modeling and energy minimization (not shown). The addition of CNGR is not expected to significantly increase the particle's hydrodynamic diameter. The present diameters are 3-4 fold larger compared with the previously published value of $6.7 \mathrm{~nm}$ determined using transmission electron microscopy [162]. However, electron microscopy can only accurately detect the particle's core, whereas dynamic light scattering measures its complete diameter, including coating.

The ionic longitudinal and transverse relaxivities $r_{1}$ and $r_{2}$ of gadolinium-labeled QDs were $7.1 \pm 0.4$ and $48.9 \pm 5.1\left(\mathrm{mM} \mathrm{Gd}^{-1} \mathrm{~s}^{-1}\right.$, respectively.

\section{Intrinsic tissue relaxation rates}

To characterize the intrinsic tissue relaxation rates and their in vivo variability, $R_{1^{-}}$ and $R_{2}$-values were measured in the liver, spleen, kidney, skeletal muscle, brain, and blood of a mouse at $7 \mathrm{~T}$ (Table 4.1). As indicated previously, the range in $R_{1}$ values was much smaller than for $R_{2}$. Furthermore, both $R_{1}$ and $R_{2}$ values correspond reasonably well with available literature values (Table 4.1 ), thereby supporting the validity of the relaxation rate measurements. 

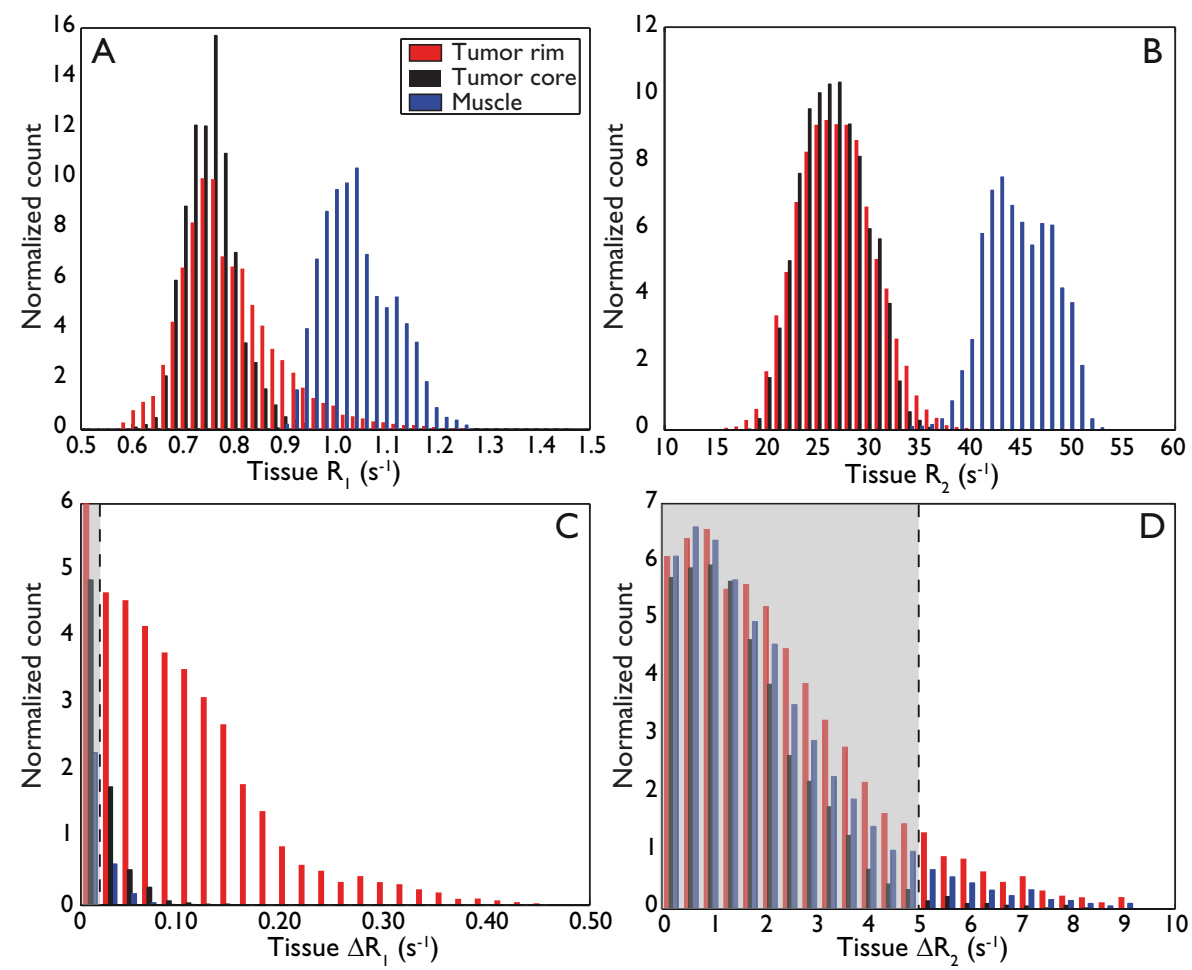

Figure 4.1: Histogram analysis for tumor rim (red), tumor core (black) and muscle tissue (blue). The relative tissue contrast between tumor and muscle was determined by measuring the median values in the histograms of pre-contrast $R_{1}(\mathrm{~A})$ and pre-contrast $R_{2}(\mathrm{~B})$. For $R_{1}$ this resulted in 0.75 and $1.05 \mathrm{~s}^{-1}$ for tumor and muscle, respectively, whereas the corresponding $R_{2}$ values were 25 and $45 \mathrm{~s}^{-1}$. In addition, panels $\mathrm{A}$ and $\mathrm{B}$ provided insight in the tissue variability in intrinsic $R_{1}$ and $R_{2}$. The histogram of $\Delta R_{1}(\mathrm{C})$ shows that changes in $R_{1}$ were most pronounced for the tumor rim. In contrast, changes in $R_{2}$ (D) were more similar for the three tissues. Furthermore, $\Delta R_{1}$ values largely exceeded the threshold as determined from Monte Carlo simulations (dashed lines), whereas $\Delta R_{2}$ values did not. Median values were calculated using all seven mice.

\section{Tumor relaxation rate mapping}

Comparable data and fit quality were obtained for the $R_{1}$ and $R_{2}$ calculations in the tumor, as shown by the similar fit errors of $7.3 \pm 3.0 \%$ and $7.8 \pm 2.6 \%$, respectively $(\mathrm{P}>0.05)$. Figure $4.1 \mathrm{~A} / \mathrm{B}$ shows histograms of the average pre-contrast $R_{1}$ and $R_{2}$ for the tumor rim, tumor core, and muscle tissue. The relative tissue contrast between tumor and muscle (Equation 4.2) was approximately twice as strong for $R_{2}$ than for $R_{1}$ (approximately 80 and $40 \%$, respectively, Figure $4.1 \mathrm{~A} / \mathrm{B}$ ), indicating that tumors are more easily identified from their surroundings using $R_{2}$-contrast. Nevertheless, the histograms show that both $R_{1}$ and $R_{2}$ values of tumor and muscle tissue were sufficiently separated to allow differentiation between the tissue types. 
Table 4.2: Average changes in relaxation rate $(\Delta R)$, percentage of enhanced voxels $\left(P_{\text {vox }}\right)$, and the quantitative contrast (QC) from $R_{1}$ and $R_{2}$ for the tumor rim, tumor core, and muscle tissue. Values are represented as median \pm standard error (calculated using all seven mice).

\begin{tabular}{lccc}
\hline & Tumor rim & Tumor core & Muscle \\
$\Delta R_{1}\left(\mathrm{~s}^{-1}\right)$ & $0.079 \pm 0.025$ & $0.025 \pm 0.0053^{\mathrm{a}}$ & $0.029 \pm 0.013^{\mathrm{a}}$ \\
$P_{\text {vox } R_{1}}(\%)$ & $42.4 \pm 10.0$ & $4.6 \pm 9.7^{\mathrm{a}}$ & $1.0 \pm 8.3^{\mathrm{a}}$ \\
$\mathrm{Q} C_{R_{1}}$ & $3.7 \pm 2.1$ & $0.06 \pm 0.5^{\mathrm{a}}$ & $0.03 \pm 0.7^{\mathrm{a}}$ \\
\hline$\Delta R_{2}\left(\mathrm{~s}^{-1}\right)$ & $5.7 \pm 0.5$ & $5.7 \pm 1.3$ & $0.0 \pm 2.9$ \\
$P_{\text {vox }}(\%)$ & $4.4 \pm 1.6$ & $0.4 \pm 2.8$ & $0.0 \pm 0.1^{\mathrm{a}}$ \\
$\mathrm{Q} C_{R_{1}}$ & $27.2 \pm 10.0$ & $2.1 \pm 2.2^{\mathrm{a}}$ & $0.0 \pm 1.8^{\mathrm{a}}$ \\
\hline
\end{tabular}

${ }^{a} \mathrm{P}<0.05$ compared with tumor rim.

Average histograms were calculated for the change in $R_{1}$ and $R_{2}$ induced by injection of cNGR-pQDs (Figure 4.1 C/D). Significantly increased values, i.e. $\Delta R_{1}$ and $\Delta R_{2}$ above the detection limit as determined by Monte Carlo simulations, could be detected for both parameters. However, $\Delta R_{1}$ largely exceeded this threshold, whereas $\Delta R_{2}$ values were only marginally larger. Furthermore, contrast agent induced $\Delta R_{1}$ values in the tumor rim, i.e. the tumor region with the highest level of angiogenic activity $[113,158]$, ranged up to $0.3 \mathrm{~s}^{-1}$ (Figure $4.1 \mathrm{C}$ ), which was considerably larger than the intrinsic variability in tumor $R_{1}$ of approximately $0.1 \mathrm{~s}^{-1}$ (Figure $4.1 \mathrm{~A}$ ). This indicates that $R_{1}$-contrast is sufficiently strong to allow detection of contrast agent induced changes. In contrast, tumor $\Delta R_{2}$ values ranged up to $7 \mathrm{~s}^{-1}$ (Figure 4.1D) and therefore fell largely within the natural variability in tumor $R_{2}$ of approximately $6 \mathrm{~s}^{-1}$ (Figure 4.1B).

The spatial distribution of contrast agent induced changes in relaxation rate was further investigated by mapping $\Delta R_{1}$ and $\Delta R_{2}$ over the tumor (Figure $4.2 \mathrm{~A} / \mathrm{B}$ ). Although both measures were predominantly enhanced at the tumor rim, statistically significant differences between tumor rim versus tumor core and muscle tissue were only found for $\Delta R_{1}$ (Table 4.2). Additionally, the percentage of significantly enhanced voxels was larger for $\Delta R_{1}$ than for $\Delta R_{2}$ (Figure 4.3, Table 4.2). This is important since a larger spatial extent of contrast enhancement over the tumor is generally easier to identify. Finally, the quantitative contrast (QC) summary measure of the intensity and spatial extent of relaxation rate changes showed statistically significant differences between tumor rim versus tumor core and muscle tissue for both $R_{1}$ and $R_{2}$ (Table 4.2). Taken together, statistically significant effects could be detected using all $R_{1}$-based parameters (i.e. $\Delta R_{1}$, percentage of enhanced voxels, and $Q C_{R_{1}}$ ), whereas for $R_{2}$, differences were only significant for $Q C_{R_{2}}$. 

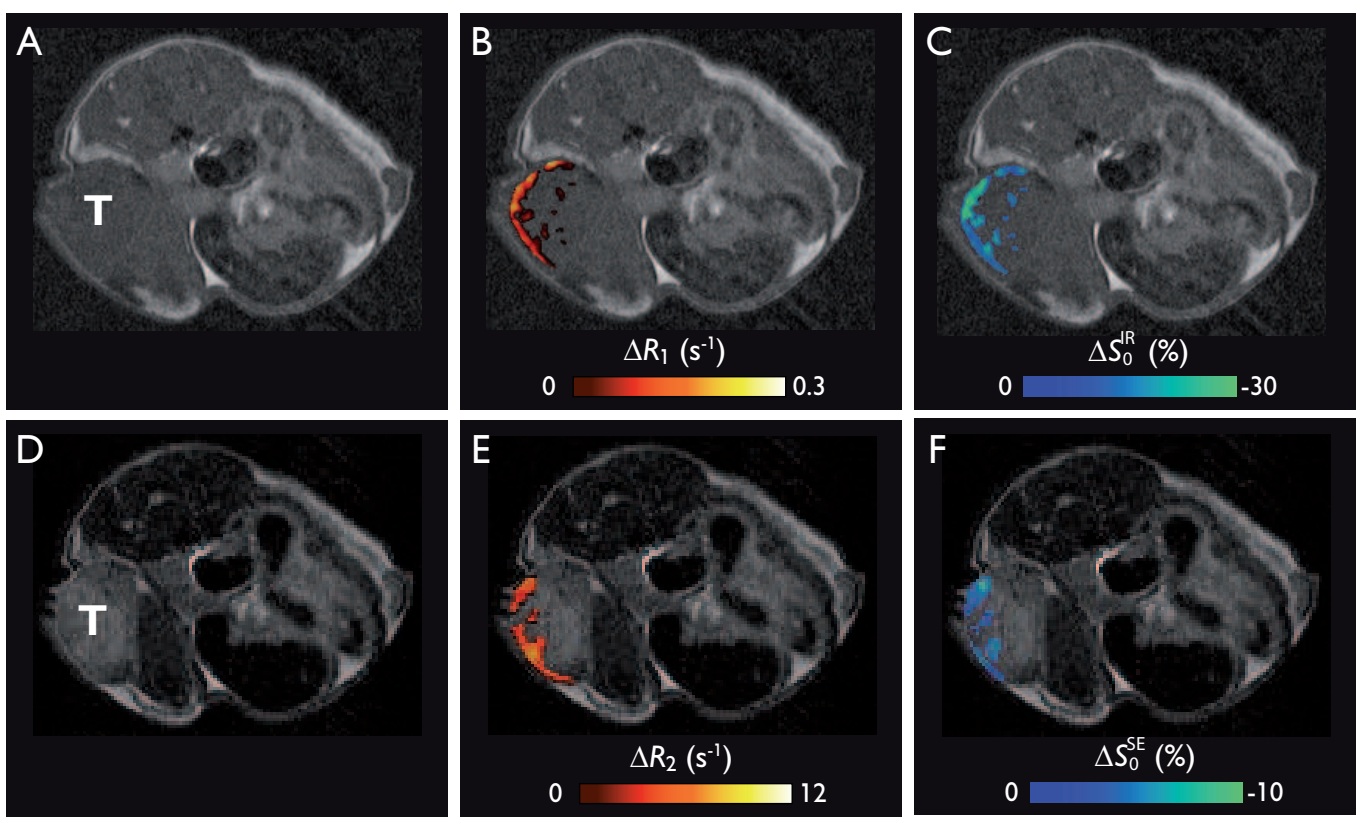

Figure 4.2: Pre-contrast $T_{1}$-weighted IR image $(\mathrm{TI}=1500 \mathrm{~ms}, \mathrm{~A})$ with color coded overlay of changes in the longitudinal relaxation rate $R_{1}(\mathrm{~B})$ and the corresponding measure of proton visibility $\Delta S_{0}^{I R}(\mathrm{C})$ for the tumor tissue $(\mathrm{T})$. Pre-contrast $T_{2}$-weighted MSME image ( $\mathrm{TE}=$ $30 \mathrm{~ms}, \mathrm{D})$ with color coded overlay of changes in the transverse relaxation rate $\Delta R_{2}(\mathrm{E})$ and $\triangle S_{0}^{S E}(F)$. The largest changes were found at the tumor periphery for all panels. Note that skeletal muscle is not shown as it was not present in the same imaging slice for this animal. All images were obtained from the same animal and slice.

The difference between $R_{1}$ and $R_{2}$ contrast enhancement was theoretically confirmed using the maximum change in signal intensity that can be obtained with IR or MSME methods. As described in Appendix C, an almost 80-fold higher sensitivity is achievable with IR than with SE (Figure C.1). Using the experimental $r_{1}$ and $r_{2}$ relaxivities, the maximum signal changes of IR and SE are approximately 7000 and 600 (arbitrary units), respectively. Although the theoretical values were calculated using in aqua relaxivities, the approximately 10 -fold difference in maximum signal changes between IR and SE corresponds well with the difference in the number of significantly enhanced voxels on IR and MSME images (Table 4.2).

Besides increasing the tissue relaxation rates, cNGR-pQDs were shown to reduce the inversion recovery scaling factor $S_{0}^{\mathrm{IR}}$, which is a measure of proton visibility $[119,154]$. This $S_{0}$-effect was also found for the $R_{2}$ determination with the MSME images (Figure 4.2C/D). Similar to $S_{0}^{\mathrm{IR}}$, changes in the scaling factor $S_{0}^{\mathrm{SE}}$ colocalized with changes in the corresponding relaxation rate. 


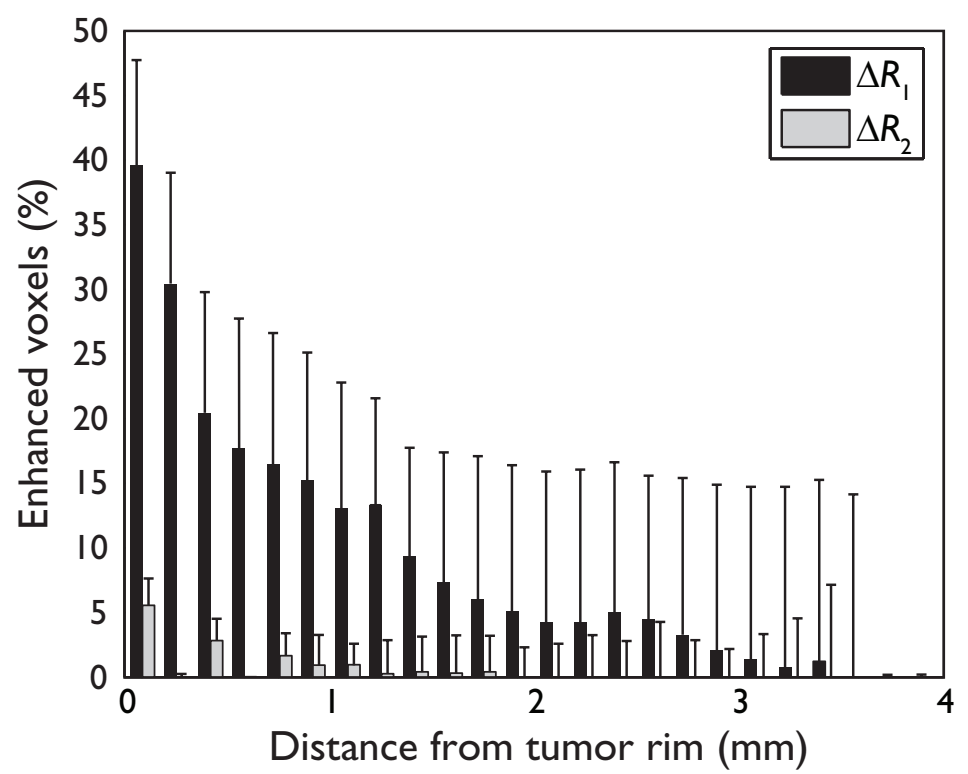

Figure 4.3: Percentage of significantly enhanced voxels at a certain distance versus the distance from the tumor rim for $\Delta R_{1}$ (black) and $\Delta R_{2}$ (grey). Although both parameters showed more enhanced voxels at the rim (i.e. distance smaller than $1 \mathrm{~mm}$ ) than in the core, clearly more significantly enhanced voxels were found for $\Delta R_{1}$ than $\Delta R_{2}$. Values are represented as median \pm standard error (calculated using all mice).

\subsection{Discussion}

Relaxation rate mapping was performed to compare $R_{1^{-}}$and $R_{2}$-characteristics for a paramagnetic quantum dot based contrast agent targeted to the angiogenic tumor vasculature. First, a statistically significant difference between tumor rim, the tumor region with the highest angiogenic activity $[113,119,158]$, versus tumor core and muscle tissue was found for all $R_{1}$-based measures $\left(\Delta R_{1}, P_{\text {vox } R_{1}}\right.$, and $\left.Q C_{R_{1}}\right)$. For $R_{2}$-based measures, differences only reached statistical significance for $Q C_{R_{2}}$. Moreover, differences between tumor rim, tumor core, and muscle were considerably higher for $R_{1}$, suggesting that $R_{1}$ may allow differentiation between tissues with distinct levels of angiogenesis, whereas $R_{2}$ does not. Second, although the majority of significantly enhanced voxels was located at the tumor rim for both $\Delta R_{1}$ and $\Delta R_{2}$, contrast agent induced changes were more conspicuous on the former, as reflected in the larger number of significantly enhanced voxels. Third, the range of pQD-induced $R_{1}$-changes in the tumor rim was approximately three times larger than the spatial heterogeneity in pre-contrast tumor $R_{1}$, indicating that contrast agent induced changes can be accurately detected using $\Delta R_{1}$. In contrast, although significant changes in $R_{2}$ were found (i.e. above threshold), $\Delta R_{2}$ values fell mainly within the inherent $R_{2}$ variability, indicating that contrast agent induced $\Delta R_{2}$ is too small to allow accurate detection. Fourth, the relative tissue contrast between tumor and 
muscle tissue was shown to be approximately two times higher for intrinsic tissue $R_{2}$ than for $R_{1}$, supporting that $T_{2}$-weighted imaging is more suitable for tumor localization. Moreover, bright appearing edema on $T_{2}$-weighted images strongly helps for tumor detection. This finding is in correspondence with clinical tumor diagnostic imaging protocols. Taken together, changes in both $R_{1^{-}}$and $R_{2}$-based measures could be detected upon administration of cNGR-labeled pQDs. However, $R_{1}$-based measures were more suitable for both the localization and quantification of contrast agent induced changes in relaxation rate at 7 Tesla, as demonstrated by theoretical and experimental analysis.

The presented $R_{1}$ results are possibly favored by the smaller voxel size of the IR images compared with the MSME images. On the one hand this allowed more accurate rim localization in the former, but on the other hand may result in a lower signal-to-noise ratio. Although the IR series consisted of a smaller number of data points than the MSME ( 6 and 8 points, respectively), the similar fit errors for the $R_{1}$ and $R_{2}$ measurements indicate that the observed differences between $R_{1^{-}}$and $R_{2^{-}}$ based measures cannot be attributed to experimental errors. Since the differences were relatively large, generally the same results are expected when comparable spatial resolutions and imaging times were used for the IR and MSME experiments. In addition, larger voxels are usually applied in clinical $T_{2}$-weighted imaging to compensate for the loss in signal-to-noise-ratio when compared with $T_{1}$-weighted imaging.

Besides $R_{1}$ and $R_{2}$ effects, intravascular contrast agents can have a considerable effect on the susceptibility. Although QDs were indeed found to have an effect on the apparent transverse relaxation rate $R_{2}^{*}$ (Table B.1), this parameter does not only depend on contrast agent presence, but also on local field inhomogeneities. Quantification of $\mathrm{pQD}$-induced changes in $R_{2}^{*}$ were therefore not included in the present study, as it might have been influenced by susceptibility artifacts related to for instances, air-tissue interfaces.

An ultimate goal of molecular MRI is the non-invasive in vivo quantification of contrast agent binding and/or expression levels of the targeted biomarker. Besides measurement of the relaxation rates, this would require knowledge of in vivo concentrations, relaxivities, and pharmacokinetic behavior of the contrast agent. Currently, no methods are available in the literature to reliably measure these parameters in vivo in inherently heterogeneous tumor tissue. In addition, cell experiments to estimate the relaxivity of bound cNGR-pQDs cannot be applied since CD13 exists in at least five different isoforms, of which only one binds cNGR [140]. The structural properties of these isoforms are currently unknown. Furthermore, it has been proposed that glycosylation and/or conformational changes of CNGR are required for binding to CD13 [185], modifications which likely occur only in vivo and not in vitro.

In conclusion, relaxation rate mapping demonstrated that significant changes in both $R_{1}$ and $R_{2}$ could be detected in tumors upon intravascular administration of cNGR-labeled pQDs at a magnetic field strength of 7 Tesla. However, $R_{1}$-based measures allowed a more accurate localization and quantification of contrast agent induced changes in relaxation rate than $R_{2}$-based measures. 


\section{Acknowledgements}

The authors thank Sander Langereis, Jeanette Smulders and Thea Haex for the ICP-MS analysis, Ludwig Dubois for in vitro growth of the tumor cells, and Roel Straathof for assistance with the dynamic light scattering experiments. 



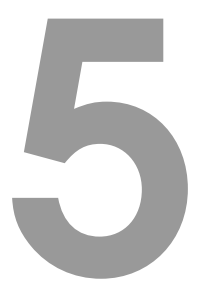

\section{Pharmacokinetics of contrast agents targeted to the tumor vasculature in molecular MRI}

Molecular MRI is increasingly used to investigate tumor angiogenic activity noninvasively. However, the pharmacokinetic behavior and tumor penetration of the often large contrast agent particles is thus far unknown. Here, pharmacokinetic analysis of cyclic asparagine-glycine-arginine (cNGR) labeled paramagnetic quantum dots (pQDs) was developed to quantify the contrast agent's homing efficacy to activated endothelial cells of angiogenic tumor vessels using DCE-MRI. The tripeptide cNGR homes to CD13, an aminopeptidase which is overexpressed on activated endothelial cells of tumor vessels. A two-compartment pharmacokinetic model, comprising the blood space and the endothelial cell surface, was defined to determine the association constant $K_{\mathrm{a}}$, the dissociation constant $k_{\mathrm{d}}$, and the fractional plasma volume $v_{\mathrm{p}}$ from the time-series data. Magnitude and spatial distribution of the pharmacokinetic parameters were compared for cNGR-labeled and unlabeled pQDs. The tumor tissue area with significant $K_{a}$ values was approximately twice as large for cNGR-pQDs compared to unlabeled $p Q D s(P<0.05)$, indicating more contrast agent binding for cNGR-pQDs. Using cNGR-pQDs, a two-fold larger area with significant $K_{a}$ was also found for the highly angiogenic tumor rim compared with the tumor core $(P<0.05)$. It was furthermore found that both contrast agents perfused the tumor at all depths, thereby providing unequivocal evidence that rim/core differences can indeed be ascribed to a higher level of angiogenesis in the rim. In conclusion, molecular DCE-MRI with two-compartment pharmacokinetic modeling provides unique information on contrast agent delivery as well as angiogenic activity in tumors.

Contrast Media and Molecular Imaging. 2009, accepted 


\subsection{Introduction}

Tumor angiogenesis is a pathological process that is well-studied using perfusion $[22,64,65]$ and molecular in vivo magnetic resonance imaging (MRI) methods [19, $110,113,115,117,124,186]$. Perfusion MR, usually performed by dynamic contrastenhanced MRI (DCE-MRI), is becoming a clinical standard for diagnosing tumor angiogenesis and for monitoring anti-cancer therapies [22]. In DCE-MRI, the wash-in and wash-out of a small contrast agent are dynamically recorded. As tumor angiogenesis is characterized by highly vascularized tissue regions, angiogenic activity can be inferred from an abnormally rapid signal intensity increase immediately after the arrival of the contrast agent. In molecular MRI, targeted contrast agents are used which home to overexpressed biomolecules that are primarily located on activated endothelial cells of tumor microvessels. Due to the inherent low sensitivity of molecular MRI to detect sparse epitopes, relatively large contrast agent particles (typically $10-300 \mathrm{~nm}$ ) are required, carrying multiple gadolinium chelates and highaffinity targeting ligands to increase contrast agent relaxivity and binding efficacy, respectively [19].

MRI responses induced by contrast agents targeted to activated endothelial cells have already been described in terms of differences in signal intensity $[22,64,65]$ and relaxation times $[110,119]$. In these studies, the strongest increases were predominantly found at the tumor rim, the area with the highest level of angiogenic activity [158]. However, it is thus far unknown if large contrast agent particles can actually penetrate into the deeper tumor regions, or whether they are not able to enter these tissue regions due to high interstitial pressures in the tumor core or absence of functional vasculature. Additional factors that affect contrast agent delivery include particle size, hydrophilicity, charge, stability, clearance rate, and microvascular permeability and flow $[187,188]$. Therefore, molecular MR images should ideally provide information on both contrast agent delivery (i.e. perfusion) and targeting efficacy. This may facilitate the quantitative interpretation of molecular MR images in low-flow areas such as the tumor core [188].

In the present study, molecular MRI and DCE-MRI of tumor angiogenesis were combined. A two-compartment pharmacokinetic model was developed to quantify the exchange between blood plasma and activated endothelial cells of the previously developed cyclic asparagine-glycine-arginine (cNGR) labeled paramagnetic quantum dots (cNGR-pQDs, Chapter 3). QDs were chosen as carrier particle due to their excellent fluorescent properties, which allowed microscopic validation. The cNGR motif was shown to home specifically to CD13, a transmembrane glycoprotein that is upregulated on endothelial cells of angiogenic vessels [136, 140,141, 143, 189, 190]. The presented method quantifies the association and dissociation constants, and plasma concentration of targeted and non-targeted control contrast agent particles. Furthermore, it reveals to which regions of the tumor the contrast agent is delivered. Molecular DCE-MRI can therefore be applied to image contrast agent homing 
efficacy to angiogenic vessels and to measure the perfusion of targeted contrast agents in tumors. The method is especially suited to study contrast agent homing to intravascular targets, since contrast agent binding is expected to occur within a clinically relevant imaging time frame ( $<30$ minutes), due to the easy accessibility of the endothelial cell targets via the blood stream.

\subsection{Methods}

\section{Contrast agents}

Streptavidin-coated cadmium/selenium quantum dots (585 nm emission wave length, $1 \mu \mathrm{M}$ solution in borate buffer $\mathrm{pH}$ 8.3) were purchased from Invitrogen (Breda, The Netherlands). Each cNGR-pQD dose was prepared freshly by mixing $100 \mu \mathrm{L}$ QDs with biotinylated cNGR peptide and biotinylated Gd-DTPA-wedge, a poly(lysine) dendritic construct containing 8 Gd-DTPA moieties, in a molar ratio of 1:6:24, as described in Section 3.2. Unlabeled (non-targeted) pQDs were prepared similarly, without addition of the ligand. Biotinylated CNGR and Gd-DTPA-wedge were synthesized using tBoc solid phase peptide synthesis [147-149]. Each particle contained a maximum of $192 \mathrm{Gd}-\mathrm{DTPA}$ groups, resulting in a $\mathrm{Gd}$ concentration of $0.2 \mathrm{mM}$. The total administered contrast agent dose was approximately $0.5 \mu \mathrm{mol} \mathrm{Gd} / \mathrm{kg}$ body weight. The ionic $T_{1}$ relaxivity was $7.1(\mathrm{mM} \mathrm{Gd})^{-1} \mathrm{~s}^{-1}$ at 7 Tesla (Chapter 3).

\section{Animal model}

All experiments were approved by the institutional ethics committee on the care for laboratory animals. Tumors were induced subcutaneously in the flank of male Swiss $\mathrm{nu} / \mathrm{nu}$ mice by injection of $1.5-3.0 \times 10^{6}$ human colorectal adenocarcinoma cells (LS174T, American Type Culture Collection CL-188, Rockville, MD). Mice were subjected to the MRI experiment when tumors exceeded $1.0 \mathrm{~cm}^{3}$ (approximately $2-3$ weeks after injection). Seven mice were included per contrast agent group. Different mice were used for targeted and control contrast agents to allow microscopic validation. In addition, pQDs were shown to accumulate mainly in the liver and spleen (Figure 3.4), and it is unknown how (partial) saturation of these organs with a first dose of pQDs might effect the pharmacokinetics of a second dose.

Mice were anesthetized using 1-2\% isoflurane (Abbott Laboratories Ltd, Queensborough, UK) in medical air. Analgesia was induced by $0.1 \mathrm{mmol} / \mathrm{kg}$ buprenorphine s.c. (Temgesic ${ }^{\circledR}$, Schering-Plough, Utrecht, The Netherlands). Subsequently, an infusion line was placed in the jugular vein for contrast agent injection during the MRI experiment. Mice were placed prone in a dedicated animal holder with built-in anesthesia mask. Body temperature was maintained at physiological levels using a warm 
water heating pad. Respiration rate and body temperature were constantly monitored using an MR compatible small animal monitoring system (SA Instruments, Stony Brook, NY). After MRI, mice were sacrificed by cervical dislocation. Tumors were excised and vascular endothelial cells were stained using endothelial cell-specific antiCD31-FITC for subsequent investigation by two-photon laser scanning microscopy (TPLSM, see Section 3.2 for details).

\section{MRI protocol}

All images were acquired with a quadrature volume resonator with a $3.5 \mathrm{~cm}$ inner diameter on a 7 Tesla Bruker Biospec 70/30 USR (Bruker Biospin, GmbH, Ettlingen, Germany) using the BGA12-S mini imaging gradient (maximum gradient strength $720 \mathrm{mTm}^{-1}$, slew rate $6000 \mathrm{Tm}^{-1} \mathrm{~s}^{-1}$ ), interfaced to an AVANCE II console.

Tumors were localized using a $T_{2}$-weighted spin echo sequence (TR $4200 \mathrm{~ms}$, TE $37.5 \mathrm{~ms}$ ). $T_{2}$-weighted images also served for tumor delineation from surrounding tissue and edema. Dynamic multi-slice gradient echo images were recorded using TR $100 \mathrm{~ms}$, TE $2 \mathrm{~ms}$, flip angle 35\%, and a $128 \times 128$ matrix. The field-of-view was $4.0 \times 4.0 \mathrm{~cm}^{2}$, resulting in a $0.31 \times 0.31 \mathrm{~mm}^{2}$ spatial resolution. Depending on tumor size and orientation, 11 to 22 axial slices (mean 15) of $1.2 \mathrm{~mm}$ thickness were recorded. In total, 70 dynamic phases with a $13 \mathrm{~s}$ temporal resolution were acquired. Injection of $120 \mu \mathrm{L}$ contrast agent was started at phase 11 , directly followed by a $50 \mu \mathrm{L}$ saline flush.

A competition experiment was performed in five additional tumor-bearing mice to further investigate the interaction of cNGR-pQDs with angiogenic tumor vessels. Here, a 1000-fold excess of free cNGR (i.e. not bound to a QD particle), was injected 10 minutes after administration of cNGR-pQDs, as described in Section 3.2. Dynamic imaging was performed as outlined above, except that the total imaging time was increased to 35 minutes.

\section{Two-compartment pharmacokinetic model}

Using TPLSM, cNGR-pQDs and unlabeled pQDs were previously found to minimally extravasate into the extracellular extravascular space or internalize into endothelial cells (Figure 5.1A, Chapter 3 ). This limits the number of accessible compartments to two: the endothelial cell layer of the vessel wall and the intravascular space. Figure 5.1B shows a schematic representation of the resulting model. Contrast agent binding to endothelial cells is governed by the association constant $k_{a}\left(\mathrm{~min}^{-1}\right)$. The reversed process of contrast agent dissociation and release into the blood plasma is characterized by the constant $k_{d}\left(\mathrm{~min}^{-1}\right)$. A detailed mathematical description of the two-compartment pharmacokinetic model is given in Appendix D. In brief, the total tumor contrast agent concentration $C_{T}$ at any time $t$ is the sum of the intravascular and endothelial cell layer contributions, which can be described by: 

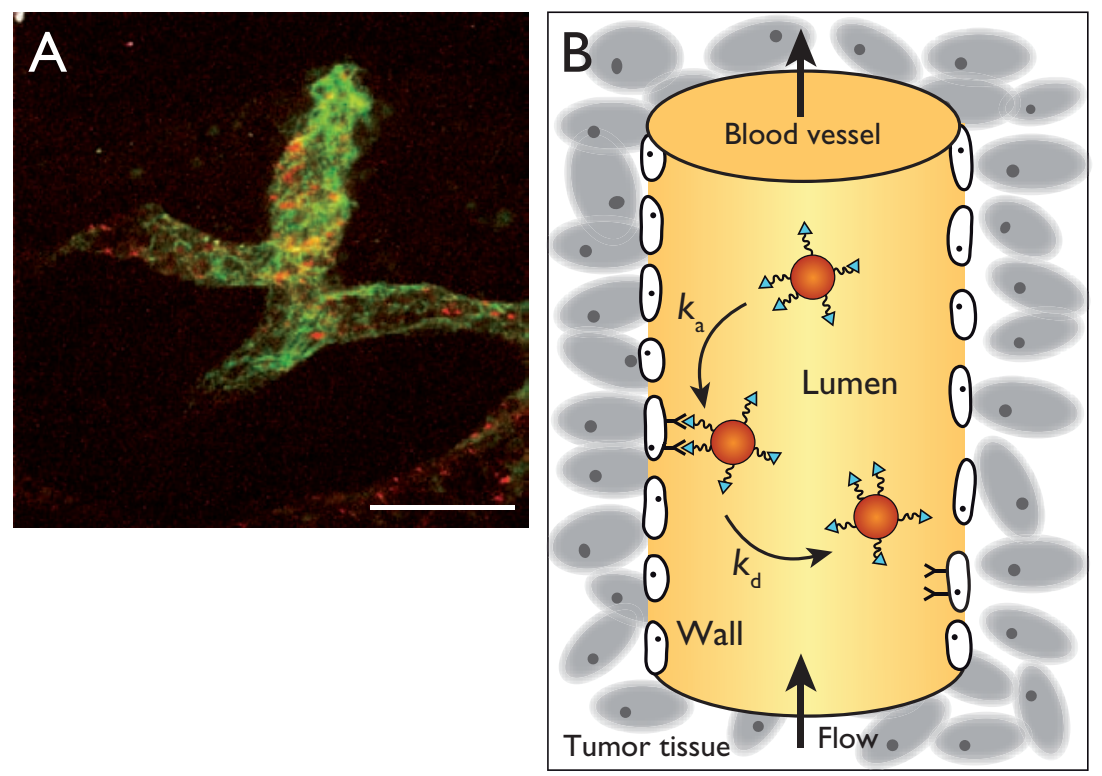

Figure 5.1: A) Ex vivo TPLSM image obtained in an intact murine tumor. The image shows colocalization of cNGR-pQDs (red) with tumor endothelial cells (green), indicating contrast agent binding to the activated endothelial cells. Furthermore, no extravasation or internalization of cNGR-pQDs is observed. Details on TPLSM data acquisition and processing have been described in Section 3.2. Bar: $50 \mu \mathrm{m}$. B) Schematic representation of the two-compartment model for targeted molecular MR contrast agents. The intravascular contrast agent binds to the activated endothelial cells with an association constant $k_{\mathrm{a}}$. The rate of dissociation is described by the constant $k_{d}$.

$$
C_{\mathrm{T}}(t)=v_{\mathrm{p}} C_{\mathrm{p}}+K_{\mathrm{a}} \int_{0}^{t} C_{\mathrm{p}}\left(t^{\prime}\right) e^{-k_{\mathrm{d}}\left(t-t^{\prime}\right)} \mathrm{d} t^{\prime}
$$

Here, $v_{p}$ and $C_{p}$ are the fractional plasma volume and the plasma contrast agent concentration, respectively. $C_{p}(t)$ is also known as the vascular input function (VIF). The parameter $K_{a}$ is defined as the product of the fractional vessel wall volume $v_{w}$ and the association constant $k_{a}$, thus $K_{a}=v_{w} k_{a}$.

\section{Image data analysis}

All data analysis was performed in the Matlab programming environment (The MathWorks, Natick, MA), unless stated otherwise. Regions of interest (ROIs) were defined manually in MRIcro [152]. Dynamic data were spatially coregistered using the rigid body transformation algorithm in SPM2 (Wellcome Trust Centre for Neuroimaging, London, UK) to correct for possible animal motion during data acquisition. Next, Gaussian in-plane image smoothing was performed in SPM2 (kernel full-width-at-half-maximum $0.8 \times 0.8 \mathrm{~mm}^{2}$ ). 
The relative signal intensity changes $\Delta S(t)$ were calculated per voxel as $S(t) / S_{\text {pre }}$ -1 with $S_{\text {pre }}$ the mean signal intensity of the pre-contrast phases. Based on the low contrast agent dose and in vivo concentrations (estimated maximum plasma Gd concentration is $10 \mu \mathrm{M}), \Delta S(t)$ was presumed to be linearly related to changes in contrast agent concentration. Differences in $r_{1}$ relaxivity between bound and unbound contrast agent were neglected. The VIF $C_{p}(t)$ was measured in a large vessel (aorta/vena cava) and subsequently multiplied by (1-Hct), with a previously determined hematocrit Hct of 0.48 [158]. Noise reduction of the plasma and tissue time curves was achieved using a Savitsky-Golay filter with a polynomial order and frame size of 3 and 13, respectively [191]. Contrast agent plasma half life was determined numerically by fitting the VIFs to a bi-exponential decay function. The pharmacokinetic parameters $K_{\mathrm{a}}, k_{\mathrm{d}}$, and $v_{\mathrm{p}}$ were calculated on a voxel-by-voxel basis by fitting the tissue curves with Equation 5.1 using the Levenberg-Marquardt numerical optimization algorithm. Voxels with a fit-error greater than $50 \%$ were excluded from further analysis [158].

Intratumoral differences were investigated by defining the tumor rim as an approximately $1 \mathrm{~mm}$ (3-4 pixels) thick peripheral zone, as described in Chapter 3. The tumor core was defined as the difference between whole tumor and tumor rim ROls. A single voxel contour was drawn around the tumor to calculate the shortest distance to the tumor periphery for each voxel.

All ROIs were drawn by one observer, who was not blinded to the contrast agent groups. However, bias was excluded by drawing all ROls on pre-contrast $T_{2}$-weighted images only (not on e.g., a $K_{\mathrm{a}}$-map) covering the entire tumor (no selection of specific slices or regions).

Histogram analysis was performed to investigate the distributions of $K_{a}, k_{d}$, and $v_{p}$ over the tumor. Based on these histograms, (empirical) thresholds were set to maximize the differences between cNGR-pQDs and unlabeled pQDs, and to exclude bins with high standard errors (Figure 5.5, vide infra). The percentage of voxels $\left(P_{\text {vox }}\right)$ with $K_{\mathrm{a}}$ above the threshold was calculated as a measure of the spatial extent of contrast agent binding. $P_{\text {vox }}$ values for $k_{d}$ and $v_{p}$ were calculated similarly.

\section{Statistical analysis}

All values are presented as mean \pm standard error. Statistical analyses were performed using Student's t-tests in SPSS 16.0 (SPSS, Chicago, III). P $<0.05$ was considered statistically significant; $\mathrm{P}<0.10$ was considered a trend towards statistical significance. 

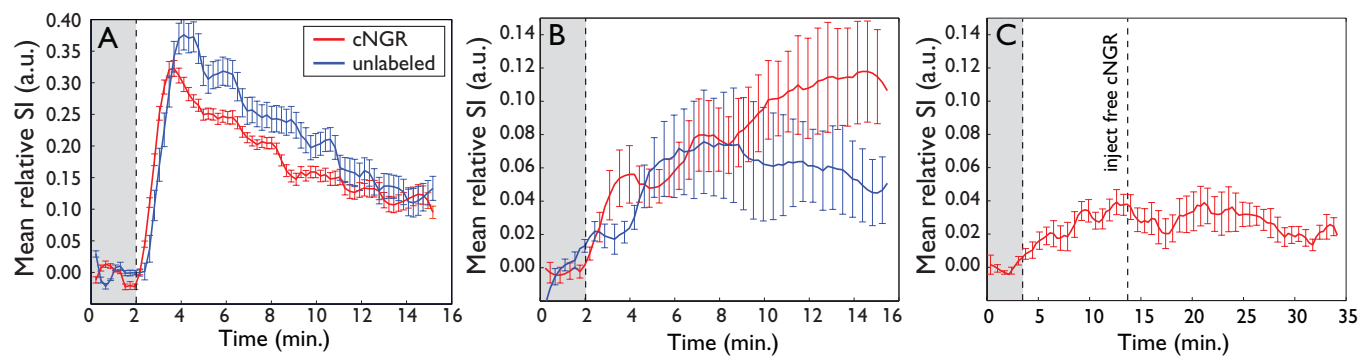

Figure 5.2: A) Averaged vascular input functions for cNGR-pQDs $(n=7)$ and unlabeled pQDs $(n=7)$. B) Averaged dynamic time curves recorded in the tumor rim of mice receiving cNGRpQDs or unlabeled pQDs. C) Average time curves for the dynamic competition experiment of cNGR-pQDs with a 1000-fold excess of free cNGR recorded in the tumor rim. Free cNGR was injected 10 minutes after cNGR-pQD administration. After an initial rise in signal intensity upon administration of cNGR-pQDs, the signal intensity decreased upon injection of free $c N G R$, indicative of effective competition. The gray-shaded areas indicate pre-contrast values. Dynamic time curves for cNGR-pQDs and unlabeled pQDs are depicted in red and blue, respectively. For clarity, error bars are plotted for every other data point in panels $\mathrm{B}$ and $C$.

\subsection{Results}

\section{Contrast agent plasma half life}

Average VIFs for cNGR-pQDs and unlabeled pQDs are shown in Figure 5.2A. The plasma half lives of cNGR-pQDs and unlabeled pQDs were $7.1 \pm 1.5$ and $7.1 \pm 1.4$ minutes, respectively $(P=0.5)$, indicating similar systemic behavior.

\section{Tissue time curves}

Average time curves obtained in the tumor rim for cNGR-pQDs and unlabeled pQDs are shown in Figure 5.2B. Upon injection of cNGR-pQDs, a continuous rise in the relative signal intensity was observed, indicative of contrast agent accumulation. For unlabeled pQDs, a comparable initial rise of typically $5 \%$ was observed, which leveled off after 6-8 minutes and decreased thereafter.

For the competition experiment of cNGR-pQDs with excess free cNGR, an increase in relative signal intensity was observed after injection of cNGR-pQDs (Figure 5.2C). However, upon intravenous administration of free cNGR, the relative signal intensity decreased, indicating efficient competition of bound cNGR-pQDs with free cNGR (Figure 5.2C). 


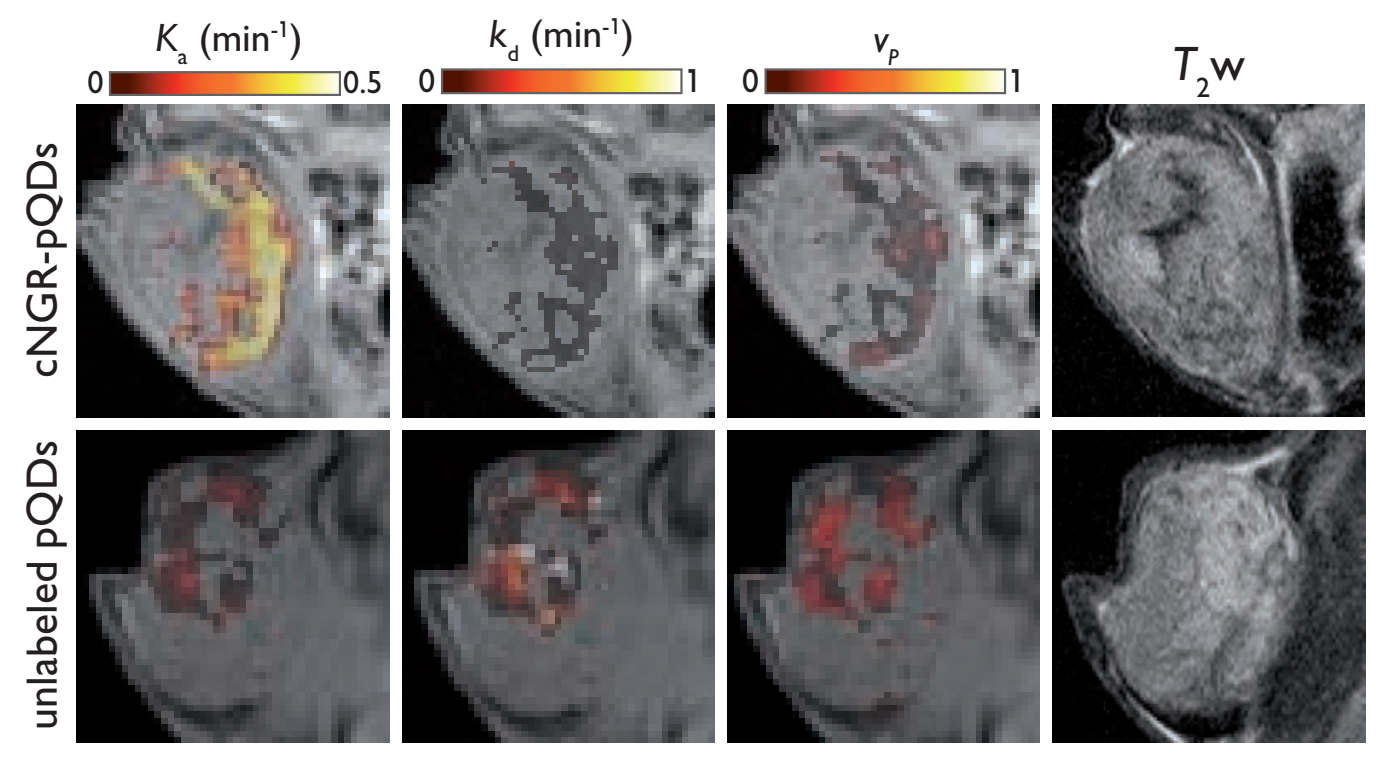

Figure 5.3: Sections of dynamic contrast-enhanced MR images showing subcutaneous tumors with color-coded overlays of the association parameter $K_{\mathrm{a}}$ (first column), the dissociation constant $k_{d}$ (second column), and the fractional plasma volume $v_{p}$ (third column) for cNGRpQDs (top row) and unlabeled pQDs (bottom row). The fourth column shows identical excerpts of the $T_{2}$-weighted images for anatomical reference.

\section{Two-compartment pharmacokinetic modeling}

Figure 5.3 shows DCE-MRI images with color-coded overlay of the association parameter $K_{\mathrm{a}}$, the dissociation constant $k_{\mathrm{d}}$, and the fractional plasma volume $v_{\mathrm{p}}$ for tumors of mice injected with cNGR-pQDs or unlabeled pQDs. These images suggest a higher $K_{a}$, a lower $k_{d}$, and a lower $v_{p}$ for cNGR-pQDs compared with unlabeled pQDs. In addition, images in Figure 5.3 show a stronger enhancement in the tumor rim than in the core for cNGR-pQDs, which concurs with previous studies $[19,110,113,115,119,158]$. For unlabeled pQDs, a more heterogeneous enhancement pattern was found over the tumor.

\section{Tumor penetration}

To investigate the tumor penetration of the contrast agents, the distance to the tumor periphery was calculated for each voxel. Next, the percentage of voxels with a significant $K_{a}$ value was plotted as a function of this distance (Figure 5.4). Although the percentage of voxels was approximately two-fold higher for cNGR-pQDs than for unlabeled pQDs, both contrast agents showed significant enhancement over tumor rim and core regions, indicating that both particles can perfuse the tumor to its full depth. 


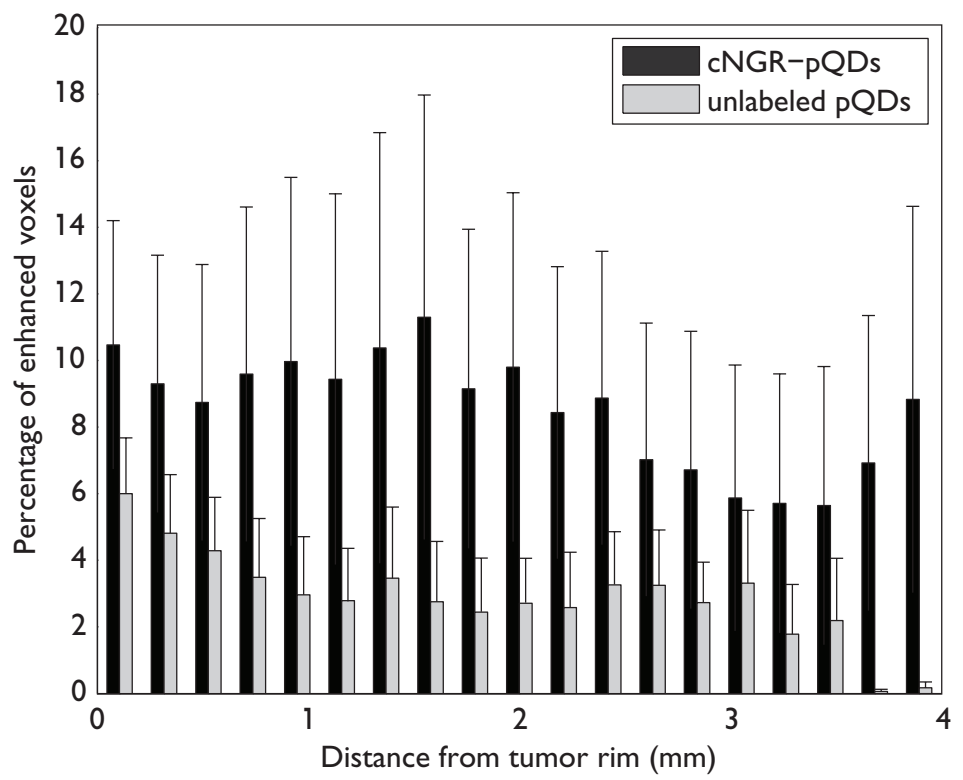

Figure 5.4: Percentage of voxels with $K_{a}$ above the empirical threshold value as defined in Figure 5.5, versus the distance to the tumor periphery for cNGR-pQDs (black bars) and unlabeled pQDs (grey bars). In general, the tumor rim contains voxels with a distance smaller than $1.0 \mathrm{~mm}$, whereas the tumor core comprises distances larger than $1.0 \mathrm{~mm}$.

\section{Contrast agent association}

The histogram of the association parameter $K_{a}$ (Figure 5.5A) shows higher $K_{a}$ values for cNGR-pQDs than for unlabeled pQDs. Next, mean $K_{a}$ values and the percentage of voxels $P_{\text {vox }}$ above the threshold of $0.1 \mathrm{~min}^{-1}$ were calculated for the two contrast agents. In the angiogenic tumor rim, $P_{\text {vox }}$ was almost two-fold higher for cNGRpQDs compared with unlabeled pQDs (Table 5.1). Additionally, $P_{\text {vox }}$ was significantly different between rim and core for both contrast agents.

\section{Contrast agent dissociation}

Figure 5.5B shows a histogram of the dissociation constant $k_{d}$. The highest values were mostly found for unlabeled pQDs. Besides the lower boundary of $0.1 \mathrm{~min}^{-1}$, an additional upper boundary was set to $3.0 \mathrm{~min}^{-1}$ to exclude unrealistically high values (mainly small, isolated groups of voxels located at tissue interfaces). $P_{\text {vox }}$ was significantly lower for cNGR-pQDs than for unlabeled $p Q D$ s in the tumor core (Table 5.1). Compared with unlabeled pQDs, a lower mean $k_{d}$ was found for cNGRpQDs in both tumor rim and core. 
Table 5.1: Summary of the percentage of enhanced voxels $\left(P_{\text {vox }}\right)$ and of the pharmacokinetic parameters $K_{\mathrm{a}}, k_{\mathrm{d}}$, and $v_{\mathrm{p}}$, for cNGR-pQDs and unlabeled pQDs for the tumor rim and core. Only voxels with parameter values above the indicated empirical threshold values were included. Note that $P_{\text {vox }}$ differs for $K_{\mathrm{a}}, k_{\mathrm{d}}$, and $v_{\mathrm{p}}$ since different optimal cut-off values were applied for the three parameters based on Figure 5.5.

\begin{tabular}{|c|c|c|c|c|}
\hline & \multicolumn{4}{|c|}{$K_{a}>0.1 \mathrm{~min}^{-1}$} \\
\hline & \multicolumn{2}{|c|}{$P_{\text {vox }}(\%)$} & \multicolumn{2}{|c|}{$K_{a}\left(\min ^{-1}\right)$} \\
\hline & RIM & CORE & RIM & CORE \\
\hline cNGR & $11.8 \pm 4.7^{a, b}$ & $6.5 \pm 4.2$ & $0.26 \pm 0.07$ & $0.39 \pm 0.23$ \\
\hline \multirow[t]{4}{*}{ Unlabeled } & $5.7 \pm 1.7^{\mathrm{b}}$ & $2.7 \pm 1.6$ & $0.33 \pm 0.08$ & $0.34 \pm 0.11$ \\
\hline & \multicolumn{4}{|c|}{$0.1<k_{\mathrm{d}} 3.0 \mathrm{~min}^{-1}$} \\
\hline & \multicolumn{2}{|c|}{$P_{\text {vox }}(\%)$} & \multicolumn{2}{|c|}{$k_{d}\left(\min ^{-1}\right)$} \\
\hline & RIM & CORE & RIM & CORE \\
\hline cNGR & $4.3 \pm 2.0^{c}$ & $2.0 \pm 1.1^{\mathrm{a}}$ & $0.29 \pm 0.09$ & $0.18 \pm 0.05$ \\
\hline \multirow[t]{4}{*}{ Unlabeled } & $7.3 \pm 5.7$ & $5.7 \pm 3.2$ & $0.40 \pm 0.10$ & $0.34 \pm 0.09$ \\
\hline & \multicolumn{4}{|c|}{$v_{p}<0.2$} \\
\hline & \multicolumn{2}{|c|}{$P_{\text {vox }}(\%)$} & \multicolumn{2}{|c|}{$v_{p}$} \\
\hline & RIM & CORE & RIM & CORE \\
\hline cNGR & $7.4 \pm 2.7^{b}$ & $2.7 \pm 1.2$ & $0.35 \pm 0.02^{c}$ & $0.21 \pm 0.06$ \\
\hline Unlabeled & $8.1 \pm 3.6$ & $5.1 \pm 4.3$ & $0.34 \pm 0.04$ & $0.33 \pm 0.05$ \\
\hline
\end{tabular}

${ }^{a} P<0.05$ compared with unlabeled $p Q D s .{ }^{b} P<0.05$ compared with tumor core. ${ }^{\mathrm{C}} \mathrm{P}<0.1$ compared with tumor core.

\section{Fractional plasma volume}

Figure 5.5C shows histograms of the fractional plasma volume $v_{p}$. Administration of cNGR-pQDs mainly resulted in low $v_{p}$ values, whereas higher values were found for unlabeled $p Q D$ s. Using a threshold value of 0.2 , a statistically significant difference was found between cNGR-pQDs and unlabeled pQDs in the percentage of enhanced voxels in the tumor rim (Table 5.1). Furthermore, mean $v_{p}$ was higher in the tumor rim compared with the tumor core, as expected based on the higher microvessel density in the periphery [158].

Figure 5.5 (facing page): Histograms of the association parameter $K_{a}(A)$, the dissociation constant $k_{\mathrm{d}}(\mathrm{B})$, and the fractional plasma volume $v_{\mathrm{p}}(\mathrm{C})$ for cNGR-pQDs (black bars) and unlabeled $p Q D s$ (grey bars). Dotted lines indicate the empirical cut-off values which were chosen to maximize the differences between cNGR-labeled and unlabeled pQDs. Values were normalized to tumor volume. 

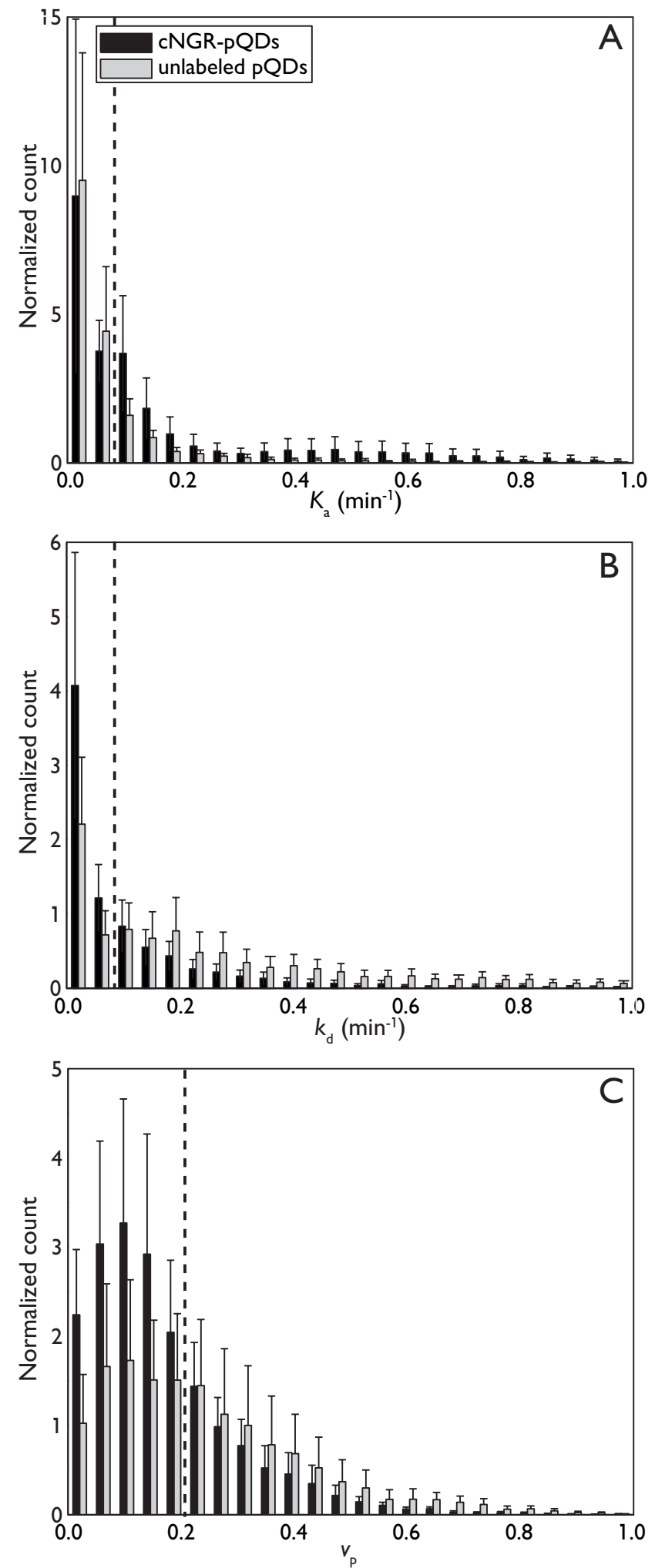


\subsection{Discussion}

\section{Current findings}

The dynamic homing behavior of cNGR-labeled paramagnetic quantum dots targeted to the angiogenic tumor vasculature was investigated using DCE-MRI. First, dynamic curves in the tumor rim showed a continuous signal increase for cNGR-pQDs (Figure 5.2B), indicating contrast agent accumulation. In contrast, unlabeled pQDs only showed a brief rise in signal intensity, which could be caused by contrast agent traversing slowly through the highly irregular tumor vasculature. The dynamic competition experiment (Figure 5.2C) showed effective competition of cNGR-pQDs with free cNGR, further supporting a specific and reversible binding of cNGR-pQDs to the angiogenic tumor vasculature.

Second, a two-compartment model (Figure 5.1B) was defined to extract the association parameter $K_{\mathrm{a}}$, the dissociation constant $k_{\mathrm{d}}$, and the fractional plasma volume $v_{p}$ from the dynamic data. Color-coded overlay images and histogram analyses of the three pharmacokinetic parameters suggested a stronger $K_{a}$ for cNGRpQDs, whereas $k_{d}$ and $v_{p}$ were highest for unlabeled pQDs (Figures 5.3 and 5.5). The higher $v_{p}$ found for unlabeled $p Q D s$ may be due to inflow effects in the 2D DCE-MRI method, and not due to an actual difference in tumor plasma volume between the experimental groups. Assuming that unlabeled pQDs move more freely through the vasculature compared with cNGR-pQDs, which may flow slower due to their avidity of binding to the vascular wall, then the inflow effect of the unlabeled pQDs would be larger and create a greater contrast, resulting in an apparent greater vascular volume. Furthermore, differences in $v_{p}$ are likely not related to differences in intravascular concentration, since this was corrected for by the application of an individually derived VIF. Significant differences in the percentage of enhanced voxels between cNGR-pQDs and unlabeled pQDs, and between tumor rim and core, were detected. This was most pronounced for the association parameter $K_{a}$ (Table 5.1).

Third, it was found that both cNGR-pQDs and unlabeled pQDs can perfuse tumor rim and core regions (Figure 5.4). This provides unequivocal evidence that the previously found rim-core differences (Chapter 3 ) can indeed be ascribed to the higher level of angiogenesis in the rim and not to differences in contrast agent perfusion.

\section{Molecular DCE-MRI}

Molecular DCE-MRI is an alternative to dynamic positron emission tomography (PET) to study contrast agent pharmacokinetics in tumors [192-194]. Advantages of PET are the much lower detection limit for sparse molecular disease markers and the easy translation of dynamic PET methodologies into clinical trials due to the often low radiotracer doses [193]. The ability of MRI to detect specific molecular 
processes in certain body regions depends on the expression level of the molecular disease markers and the ability to deliver a sufficiently high local concentration of the contrast agent. MRI has several distinct advantages. First, no ionizing radiation is involved. Second, images can be obtained in any oblique direction, which allows selection of the optimal imaging slice for each specific anatomic region. Third, MR images have a superior soft-tissue contrast and spatial resolution, which is important for the detection of anatomical landmarks, such as the mesorectal fascia in colorectal cancer. This additionally allows pixel-by-pixel pharmacokinetic analysis of the tumor at a much more detailed level than obtainable with radionuclear acquisition techniques. Fourth, molecular DCE-MRI can be combined with other imaging protocols for tumor diagnosis and therapy monitoring, including diffusion and $T_{2}$-weighted imaging.

\section{Pharmacokinetic modeling}

Molecular DCE-MRI with two-compartment pharmacokinetic modeling enabled the quantification of the dynamic targeting behavior of contrast media, thereby allowing the simultaneous investigation of contrast agent association to and dissociation from endothelial cells, as well as contrast agent delivery over the tissue. In contrast, the comparison of single pre- and post-contrast images only provides information on contrast agent uptake at a certain time-point.

Recently, Neubauer et al. used DCE-MRI and compartmental analysis to describe the pharmacokinetic behavior of perfluorocarbon nanoparticles targeted to the neovasculature of atherosclerotic lesions [195]. Besides the differences in disease model, major methodological differences with the current study are found in the timing of image acquisition. Furthermore, Neubauer et al. used a three-compartment model to describe the dynamic MRI data and measured the VIF via blood sampling. The differences in pathology, MRI protocol, and pharmacokinetic model hamper a direct comparison. However, in line with the current study, Neubauer et al. found that differences between targeted and non-targeted particles were most pronounced for the association parameter [195].

\section{Contrast agent relaxivity}

Although differences in the longitudinal relaxivity $r_{1}$ between intravascular and bound contrast agent were ignored in the present study, intratumoral differences can still be described accurately without proper knowledge of the relaxivities, since the simplification of contrast agent relaxivity affects each tumor area equally. The conclusion that both cNGR-labeled and unlabeled pQDs can perfuse the tumor at all depths therefore remains valid. 
Nevertheless, it is expected that $r_{1}$ will increase upon contrast agent binding due to reduced particle tumbling. Although this effect is not expected to be extremely large since the tumbling rate is already relatively low due to the large particle size, an assumed too low $r_{1}$ will result in an overestimation of the concentration of bound contrast agent. If the true in vivo relaxivities of bound and unbound contrast agent were known, it is therefore expected that $K_{\mathrm{a}}$ will be higher and $k_{\mathrm{d}}$ may be somewhat lower for the targeted contrast agent, and vice versa for the non-targeted contrast agent. This would consequently result in stronger differences between the two contrast agents in terms of the calculated association and dissociation constants. In addition, this may explain why the percentage of enhanced voxels $P_{\text {vox }}$ was more sensitive to detect differences between cNGR-labeled and unlabeled pQDs, than the mean pharmacokinetic parameters $K_{\mathrm{a}}, k_{\mathrm{d}}$, and $v_{\mathrm{p}}$.

\section{Compartments}

Two compartments result in a relatively simple pharmacokinetic model, but the involvement of additional compartments cannot be excluded. Based on the large intracellular spaces typically found in tumor vessels [38] and pQD-size, it is expected that pQDs may enter the extravascular space, necrotic core, and/or blood lakes. However, pQD extravasation may have been hampered by the rapid plasma clearance. For particles that do internalize and/or extravasate, the model should be adapted to include these additional compartments, likely at the expense of increased complexity and uncertainty of the fitted pharmacokinetic parameters.

To evaluate whether quantitative information from an additional compartment could be obtained by further improving the numerical fit quality of the dynamic uptake curves, a three-compartment model was defined constituting the macrovasculature (aorta/vena cava), the tumor microvascular lumen, and the tumor microvascular wall. In comparison with the two-compartment model, the three-compartment model provided a similar description of the dynamic data but did not result in a significantly better fit (data not shown).

\section{Study limitations}

A general limitation for in vivo derived pharmacokinetic parameters is the absence of an accurate validation method. Where the immunohistochemical assessment of microvessel densities is considered a surrogate validation method for permeability mapping with DCE-MRI, the microvascular density does not provide information on whether endothelial cells are activated or quiescent. Moreover, experimental data on the binding constant of cNGR for CD13 is lacking. Reliable in vitro measurement of the binding constant, using for instance surface plasmon resonance, has not been realized thus far and is actually problematic since CD13 exists in at least five different 


\subsection{DISCUSSION}

isoforms of which only the form present on angiogenic vessels binds to cNGR [140]. The structural determinants of the different isoforms are currently unknown. Furthermore, it has been proposed that the selectivity of cNGR for angiogenic endothelial CD13 requires e.g. differential glycosylation or conformational changes [185]. These adaptations are likely to occur only in vivo and not in vitro. A possible future experiment to investigate the in vivo dynamic binding characteristics in more detail would be to perform measurements using both targeted and control contrast agents in the same animal, and varying the order in which the contrast agents are administered.

The application of an individually determined VIF can introduce large uncertainties due to the limited temporal resolution, partial volume effects, or animal motion. An alternative technique to estimate the VIF is to draw blood samples and quantify contrast agent concentration via fluorescence spectroscopy. It is however not expected that this yields a significantly higher temporal resolution than currently obtained with DCE-MRI. Moreover, a VIF derived by blood sampling may not accurately describe the plasma concentration in the irregular tumor vasculature.

\section{Practical applications}

Several applications can be envisioned for molecular DCE-MRI. First, in combination with the static MRI response of the contrast agent, dynamic imaging with two-compartment modeling can provide information on angiogenic activity on both a molecular and functional level within one imaging session. This may allow more accurate diagnosis or therapy monitoring, and may provide insight into possible therapeutic mechanisms. Second, molecular DCE-MRI might be suitable to investigate the tissue distribution of nanoparticles carrying e.g. cytotoxic drugs or gene constructs. This would provide direct information on whether the drugs reach their target and on tissue retention time, which would be valuable for the development and evaluation of pharmaceutical products. Third, the presented method can be easily adapted to study the pharmacokinetics of other contrast agents targeted to, for instance, the Her-2/Neu receptor [196], tumor transglutaminase [197], or the underglycosylated mucin-1 tumor antigen [198]. However, differences in extravasation and cellular uptake of probes should be carefully investigated. For instance, EGF-receptors such as HER-2/neu internalize after binding and this should be included in the pharmacokinetic model. Fourth, knowledge of the contrast agent pharmacokinetic behavior allows calculation of the optimal time frame for post-contrast imaging, i.e. the time during which the post-contrast signal is maximal. This would also be relevant in a clinical setup, since images can be obtained directly at the most suitable time point after contrast agent injection and there is no need for multiple imaging sessions up to several hours post-contrast (see Appendix D). 



\section{6}

\section{Evaluation of MR vessel size imaging by two-photon laser scanning microscopy}

Magnetic resonance vessel size imaging (MR-VSI) is increasingly applied to noninvasively assess microvascular properties of tumors and to evaluate tumor response to anti-angiogenic treatment. MR-VSI provides measures for the microvessel radius and fractional blood volume of tumor tissue. However, data has not yet been evaluated with 3D microscopy techniques. Therefore, 3D two-photon laser scanning microscopy (TPLSM) was performed to assess microvascular radius and fractional vessel volume in tumor and muscle tissue. TPLSM data displayed a maze-like architecture of the tumor microvasculature and mainly parallel oriented muscle microvessels. For both MR-VSI and TPLSM, a larger vessel radius and fractional blood volume were found in the tumor rim than in the core. The microvessel radius was approximately 6 times larger in tumor and muscle for MR-VSI than for TPLSM. The tumor blood volume was 4-fold lower with MR-VSI than with TPLSM, whereas muscle blood volume was comparable for both techniques. Differences between the tumor rim, core, and muscle tissue showed similar trends for both MR-VSI and TPLSM parameters. These results indicate that MR-VSI does not provide absolute measures of microvascular morphology, however, it does reflect heterogeneity in microvascular morphology. Hence, MR-VSI may be used to assess differences in microvascular morphology.

Magnetic Resonance in Medicine. 2009, accepted 


\subsection{Introduction}

Tumor growth and metastasis formation are accompanied by the angiogenic expansion of the supporting microvascular network [199]. Clinical studies have shown that the immunohistochemical microvessel density (MVD) is a valuable prognostic indicator for various tumor types [4]. In addition, changes in MVD may provide a rough indication of therapeutic efficacy [4]. However, tumor MVD as assessed on biopsy samples significantly hampers longitudinal evaluation, is prone to sampling errors in heterogeneous tumors, and is discomforting for patients. Magnetic resonance vessel size imaging (MR-VSI) has been proposed as a non-invasive alternative to the histologically determined MVD, as it allows in vivo estimation of a vessel radius index and fractional blood volume in whole tissue on a voxel-by-voxel basis [58].

MR-VSI is based on the different nature of changes in the transverse relaxation rates $R_{2}$ and $R_{2}^{*}$ of blood and surrounding tissue as a function of microvascular dimensions. These changes can be induced either by endogenous contrast mechanisms, i.e. a change in blood deoxyhemoglobin concentration [200], or by exogenous intravascular contrast agents such as ultra-small superparamagnetic iron oxide (USPIO) particles [56]. In regions adjacent to large vessels, a strong increase in $R_{2}^{*}$ and only a small increase in $R_{2}$ are expected, whereas changes in $R_{2}^{*}$ and $R_{2}$ will be about the same near small vessels, because the proton diffusion path length covers the induced susceptibility gradient [200]. Therefore, the ratio of relaxation rate changes $\left(\Delta R_{2}^{*} / \Delta R_{2}\right)$ was shown to be related to the average dimensions of the microvessels $[56,200]$. This principle was extended by Troprès et al., who suggested that MR-VSI allows estimation of a tumor vessel radius index and fractional blood volume [58].

MR-VSI has been applied to assess vascular development in various pre-clinical tumors, including brain tumors $[58,59,201]$ and subcutaneously induced solid tumors [62, 202]. It allowed monitoring of therapeutic intervention [62, 202, 203] and the effects of stimulated pericyte recruitment [61] and hypoxia [204]. However, evaluation of MR-VSI with standard histology showed a systematic overestimation of the MR-derived vessel radius index with a considerable inter-study variability $[59,201,203]$. Pathak et al. developed a histological method based on tissue sectioning, three-dimensional (3D) reconstruction, and stereological principles, to yield a fractional blood volume that correlated well with MR-VSI [205]. However, the main drawbacks of histological methods for MVD and vessel size quantification include inadequate tissue sampling, non-uniform tissue shrinkage, thereby disturbing the morphological characteristics of the microvasculature $[206,207]$, incomplete immunostaining, and the lack of discrimination between functional and non-functional microvasculature. In addition, histologic preparation results in the loss of the endothelial glycocalyx, which constitutes a significant part of the total vascular volume [208], and hence, may result in an overestimation of vessel radius and volume. Other methods for estimating tumor MVD include quantitative PCR of angiogenic 
markers [209] and monitoring of ${ }^{18}$ FDG accumulation with positron emission tomography (PET) $[210,211]$. Although these methods were shown to correlate with the histologically determined MVD, and hence could potentially replace histology, the obtained results merely represent indicative values for MVD. PCR suffers from similar tissue sampling problems as histological MVD, whereas, ${ }^{18}$ FDG accumulation not solely depends on the MVD, but also on the metabolic activity of the tumor cells, which should be taken into account.

The goal of the present study was to compare MR-VSI measurements with 3D two-photon laser scanning microscopy (TPLSM). TPLSM enables optical sectioning of intact tissue with high spatial resolution $(<1 \mu \mathrm{m})$ and penetration depths of more than $200 \mu \mathrm{m}$ [212], which allows visualization and quantification of individual microvessels.

In addition, MR-VSI has been applied predominantly to assess vascular morphology in brain tumors, where the microvasculature is disorganized and the bloodbrain-barrier may affect the observed contrast between healthy and cancer tissue. Therefore, an additional goal of this study was to apply 3D TPLSM and MR-VSI to both the disorganized and the highly organized microvasculature of subcutaneous tumors and skeletal muscle tissue, respectively.

\subsection{Methods}

\section{Contrast agents}

The USPIO contrast agent Sinerem ${ }^{\circledR}$ (ferumoxtran-10) was kindly provided by Guerbet (Aulnay-sous-Bois, France). The administered dose was $200 \mu \mathrm{mol} \mathrm{Fe} / \mathrm{kg}$ body weight, as described previously [58]. USPIOs were diluted in saline to obtain a total administered volume of $100 \mu \mathrm{L}$.

For TPLSM, the vasculature was demarcated from surrounding tissue by fluorescent labeling of vascular endothelial cells. Therefore, excised tissues were incubated for 30 minutes in FITC-conjugated anti-mouse anti-CD31 ( $\alpha$ CD31-FITC, $0.5 \mathrm{mg} / \mathrm{mL}$, BD Biosciences Pharmingen, Alphen a/d Rijn, the Netherlands), diluted $20 \times$ in Hanks balanced saline solution (HBSS, pH 7.4).

\section{Spectrophotometry}

The inherent fluorescence of the USPIOs was measured using a NanoDrop ND3300 fluorospectrometer (Thermo Fisher Scientific, Waltham, MA). The potential influence of USPIOs on the fluorescence intensity of $\alpha$ CD31-FITC was analyzed in triplicate using an UVIKON 923 spectrophotometer (Bio-Tek, Kontron Instruments, Milan, Italy). The intensity of diluted $\alpha$ CD31-FITC ( $1 \mu \mathrm{M}$ in HBSS) was measured 
at wavelengths of 500 - $700 \mathrm{~nm}$ (step size $4 \mathrm{~nm}$ ) with increasing USPIO concentration $(0,2.2,8.8$, and $22 \mathrm{mM} \mathrm{Fe})$. Fluorescence resonance energy transfer from $\alpha$ CD31-FITC to USPIO was analyzed by comparing the fluorescence lifetime (SPC830, Becker \& Hickl GmbH, Berlin, Germany) of $\alpha$ CD31-FITC ( $1 \mu \mathrm{M}$ in HBSS) in the absence and presence of USPIOs (22 $\mathrm{mM} \mathrm{Fe}$ ).

\section{Animal model}

All animal experiments were approved by the institutional ethical review committee on animal experiments. Ten male Swiss nu/nu mice (Charles River, Maastricht, the Netherlands) received a subcutaneous unilateral injection of approximately $3 \times 10^{6}$ human colorectal adenocarcinoma cells (LS174T, American Type Culture Collection CL-188, Rockville, MD) in the flank. MRI examinations were performed when tumors reached a volume of about $1 \mathrm{~cm}^{3}$ (approximately 14 days after tumor induction). For TPLSM imaging, a separate group of eleven LS174T tumor-bearing mice was used because USPIOs reduced the vascular fluorescence intensity (vide infra). Mice were anesthetized using $1-2 \%$ isoflurane (Abbott Laboratories Ltd, Queensborough, UK) in medical air. An infusion line of PE-10 tubing (Smiths Medical International Ltd, Hythe, UK) was placed in the jugular vein for contrast agent injection. Subsequently, mice were placed prone in an animal holder with built-in anesthesia mask. Body temperature and respiration rate were continuously monitored via an MR compatible small animal monitoring system (SA Instruments, Stony Brook, NY). Physiological body temperature was maintained using a warm water heating pad placed over the mouse.

\section{MRI protocol}

MRI was performed on a 7 Tesla Bruker Biospec 70/30 USR scanner (Bruker Biospin $\mathrm{GmbH}$, Ettlingen, Germany), using the BGA12-S mini-imaging gradient (maximum gradient strength $720 \mathrm{mTm}^{-1}$, slew rate $6000 \mathrm{Tm}^{-1} \mathrm{~s}^{-1}$ ), interfaced to an AVANCE II console. A $3.5 \mathrm{~cm}$ inner diameter quadrature volume resonator was used for RF transmission and reception.

Tumors were localized using coronal and axial $T_{2}$-weighted spin echo images with a repetition time (TR) of $3000 \mathrm{~ms}$ and an echo time (TE) of $36 \mathrm{~ms}$. Pre- and post-contrast $R_{2}$ values were determined using a multi-slice multi-echo (MSME) spin echo sequence with increasing echo times (TR $4000 \mathrm{~ms}$, TE 10, 20.. $80 \mathrm{~ms}$ ). A $4.0 \times 4.0 \mathrm{~cm}^{2}$ field-of-view was used with a $128 \times 128$ matrix, resulting in an in-plane resolution of $0.31 \times 0.31 \mathrm{~mm}^{2}$. Slices were recorded in axial direction with a thickness of $1.2 \mathrm{~mm}$, covering the entire tumor. Depending on tumor size and orientation, 12 slices were recorded on average (ranging between 5 and 17 slices). Acquisition time of the MSME sequence was 6.5 minutes. 
Pre- and post-contrast $R_{2}^{*}$ values were measured using sequentially recorded multi-slice gradient echo (FLASH) images with increasing echo times (TR $1000 \mathrm{~ms}$, TE 2, 4, 6, 8, 10, 15, $20 \mathrm{~ms}$, flip angle $35^{\circ}$ ). Geometrical parameters were identical to those of the MSME measurements and the total acquisition time of the FLASH series was 15 minutes. Post-contrast image acquisition was started at least 5 minutes after USPIO injection to allow circulation and obtain adequate distribution.

The apparent diffusion coefficient (ADC) was determined using a diffusion weighted spin echo sequence with 6 orthogonal directions (TR $2000 \mathrm{~ms}$, TE $30 \mathrm{~ms}, \mathrm{~b}=0$ and $572 \mathrm{~s} \cdot \mathrm{mm}^{-2}$, diffusion gradient duration $8 \mathrm{~ms}$, diffusion gradient separation $13 \mathrm{~ms}$, 4 axial slices positioned around the tumor centre, $1.2 \mathrm{~mm}$ thickness with $1.2 \mathrm{~mm}$ gap, $128 \times 64$ matrix, field-of-view $4.0 \times 4.0 \mathrm{~cm}^{2}, 2$ averages, acquisition time 30 minutes). Diffusion-weighted imaging was only performed before contrast administration.

After MRI, mice were sacrificed by cervical dislocation. Tumors were excised and embedded in optimal cutting temperature compound (Sakura Finetek Europe, Zoeterwoude, the Netherlands), snap-frozen in cold 2-methyl-butane (Acros Organics, Geel, Belgium) and subsequently transferred to liquid nitrogen. The contralateral quadriceps femoris muscle was excised up to approximately $1 \mathrm{~cm}$ proximal to the knee joint and frozen likewise. Tumor and muscle tissues were stored at $-80{ }^{\circ} \mathrm{C}$ until TPLSM measurements.

\section{MRI data analysis}

All data processing was performed using customized software programmed in Matlab (The MathWorks, Natick, MA), unless indicated otherwise. Image coregistration was performed in SPM2 (Statistical Parametric Mapping, Wellcome Trust Centre for Neuroimaging, London, UK) to correct for any animal motion between the MSME and FLASH series. Images were smoothed using an in-plane Gaussian smoothing kernel with a full-width-at-half-maximum of $0.7 \times 0.7 \mathrm{~mm}^{2}$ in SPM2. Regions of interest were defined manually for tumor and muscle tissue using MRIcro [152].

Pre- and post-contrast $R_{2}$ and $R_{2}^{*}$ values were determined on a voxel-by-voxel basis by non-linear curve fitting of the signal intensity versus TE function, obtained from spin echo and gradient echo acquisitions, respectively:

$$
S(\mathrm{TE})=S_{0} \cdot e^{-\mathrm{TE} \cdot R_{2}^{(*)}}
$$

using the Levenberg-Marquardt optimization algorithm. Here, $S_{0}$ is a scaling factor depending on TR, proton density, and general scanner settings including preamplifier and receiver gain, and pulse profiles. Subsequently, $\Delta R_{2}^{(*)}$ values were calculated as $\Delta R_{2}^{(*)}=R_{2, \text { post }}^{(*)}-R_{2, \text { pre }}^{(*)}$. The thresholds for significantly positive $\Delta R_{2}^{(*)}$ values were determined using Monte-Carlo simulations, as described previously (Chapter 3 ). 
Mean ADC values of for the tumor rim, tumor core, and muscle, were calculated for each individual mouse using Paravision 4.0 (Bruker Biospin). Voxel-based ADC values were not applied for the vessel radius index calculations due to the differences in spatial resolution between the $\mathrm{ADC}$ and $\Delta R_{2}^{(*)}$ measurements. Next, the vessel radius index $R I_{M R I}$ and fractional blood volume $f B V_{M R I}$ were calculated on a voxelby-voxel basis according to Troprès et al. [58]:

$$
\begin{gathered}
\mathrm{RI}_{\mathrm{MRI}}(\mu \mathrm{m})=0.424 \cdot \sqrt{\frac{A D C}{\gamma \Delta \chi B_{0}}}\left(\frac{\Delta R_{2}^{*}}{\Delta R_{2}}\right)^{3 / 2} \\
\mathrm{fBV}_{\mathrm{MRI}}(\%)=\frac{3}{4 \pi} \frac{\Delta R_{2}^{*}}{\gamma \Delta \chi B_{0}}
\end{gathered}
$$

All parameters in equations 6.2 and 6.3 are given in normalized cgs units, with $\gamma$ being the gyromagnetic ratio, $\Delta \chi$ the USPIO-induced susceptibility difference between blood and surrounding tissue, and $B_{0}$ the main magnetic field strength. $\Delta \chi$ was set to $0.571 \mathrm{ppm}$ [58]. Only voxels with a significantly positive $\Delta R_{2}^{(*)}$ were included in the calculation. For tumor rim, core, and muscle tissue the resulting percentage of significant voxels was $59.0 \pm 6.1 \%, 57.8 \pm 5.7 \%$ and $86.1 \pm 4.2 \%$, respectively (mean \pm standard error). An upper boundary of $100 \mu \mathrm{m}$ was set for $\mathrm{RI}_{\mathrm{MRI}}$ to exclude unrealistically high values [59].

To investigate differences in $\mathrm{Rl}_{\mathrm{MRI}}$ and $\mathrm{fB} \mathrm{V}_{\mathrm{MRI}}$ between the tumor rim and core, the rim was defined manually as an approximately $1 \mathrm{~mm}$ thick peripheral zone, as described previously (Section 3.2). The tumor core was defined as the difference between whole tumor and tumor rim.

\section{TPLSM protocol}

Prior to TPLSM measurements, tumor and muscle tissue were thawed and washed with HBSS. Tumors were cut in half to resemble the central MR slices; muscle tissue was not processed. Tissues were incubated in $\alpha$ CD31-FITC, embedded in $2 \%(\mathrm{w} / \mathrm{v})$ agarose gel (Invitrogen, Breda, the Netherlands), and imaged with TPLSM. Note that due to the ex vivo labeling of the vascular endothelial cells, TPLSM detects all vessels resulting in the fractional vessel volume. In contrast, MR-VSI only detects the perfused vessels, resulting in the fractional blood volume.

TPLSM was performed as described previously at randomly selected locations within the tissue (Section 3.2). In brief, in-depth images were recorded with an Eclipse E600FN upright microscope (Nikon, Tokyo, Japan) equipped with a Radiance 2100MP optical imaging system (Bio-Rad, Hemel Hempstead, UK). Fluorophores were excited by a mode-locked Tsunami Ti:Sapphire laser (Spectra-Physics, Mountain View, CA, USA) with a central wavelength of $800 \mathrm{~nm}$ and a $120 \mathrm{fs}$ pulse width. $3 \mathrm{D}$ datasets were acquired using a $60 \times$ water-dipping objective lens (Nikon) with a 
numerical aperture of 1.00 . Fluorescence $(520-560 \mathrm{~nm})$ was detected by a photomultiplier tube (Electron Tubes, Ruislip, UK) and color-coded in green. A 512×512 matrix was applied to the $196 \times 196 \mu \mathrm{m}^{2}$ field-of-view, resulting in in-plane pixels of $0.38 \times 0.38 \mu \mathrm{m}^{2}$. The pixel dwell time was $11.8 \mu \mathrm{s}$, which together with a two-fold Kalman averaging for noise reduction resulted in an acquisition rate of $0.16 \mathrm{~Hz}$ for each of the subsequent images in the 3D dataset. The interplanar distance was 1.05 or $0.6 \mu \mathrm{m}$.

\section{TPLSM data analysis}

The acquired 3D datasets were deconvolved using AutoQuant X2 - AutoDeblur (MediaCybernetics, Bethesda, MD, USA) in 3D blind deconvolution mode, which required objective lens specifications, refractive index of the medium $(n=1.33)$, spatial parameters, and the emission wavelength maximum of FITC $(\lambda=518 \mathrm{~nm})$ to estimate the initial point spread function. Deconvolution was optimized using $4.43 \pm 0.31 \mu \mathrm{m}$ diameter fluorescent microspheres (Polysciences Inc., Warrington, PA), which were diluted $20 \times$ in $2 \%(w / v)$ hot agarose gel, homogenized, solidified at room temperature, and imaged with TPLSM. Using 10 iterations, 3D blind deconvolution reduced the microsphere volume from $100.11 \pm 3.46 \mu \mathrm{m}^{3}$ to $42.41 \pm 4.02 \mu \mathrm{m}^{3}$ (mean $\pm S D$, $n=5)$, which is not different from the theoretical volume $\left(45.52 \mu \mathrm{m}^{3}, P=0.1\right)$.

After 3D blind deconvolution of the vasculature datasets, in-plane microvessel radiuses ( $R_{\text {TPLSM }}$ ) in the tumor and muscle were determined using Image Pro Plus V6.2 (MediaCybernetics). RTPLSM values were determined manually between vessel bifurcations at 8 random locations in each 3D dataset to yield the median vessel radius.

The fractional vessel volume ( $\left.\mathrm{fV} \mathrm{V}_{\mathrm{TPLSM}}\right)$ and microvessel length (MVLTPLSM) were determined using 3D Constructor 5.1 (MediaCybernetics), as outlined in Figure 6.1. In brief, (1) the deconvolved 3D datasets (Figure 6.1 A/E) were smoothed using a $3 \times 3 \times 3$ voxel Gaussian kernel, (2) a 3D closing filter was applied to create massive cylinder-shaped structures, and (3) the dataset was convolved with an isotropic 3D Gaussian filter with dimensions of the dataset's median microvessel diameter (Figure 6.1 B/F), (4) an isosurface (Figure 6.1 C/G) was applied by means of intensity thresholding such that the applied isosurface closely matched the raw dataset (visual inspection), (5) the volume of all voxels within the isosurface was integrated and divided by the total dataset volume to yield the fVVTPLSM, (6) a skeletonization procedure was applied to yield the central lines of the microvasculature, and (7) a pruning filter was set to twice the median microvessel diameter as a restriction for the side branches. Next, the MVL TPLSM was calculated and expressed per unit of volume (Figure $6.1 \mathrm{D} / \mathrm{H}$ ). In addition, the total number of branching points in the skeletonized dataset was expressed per unit of volume to obtain the branching index BTPLSM. 


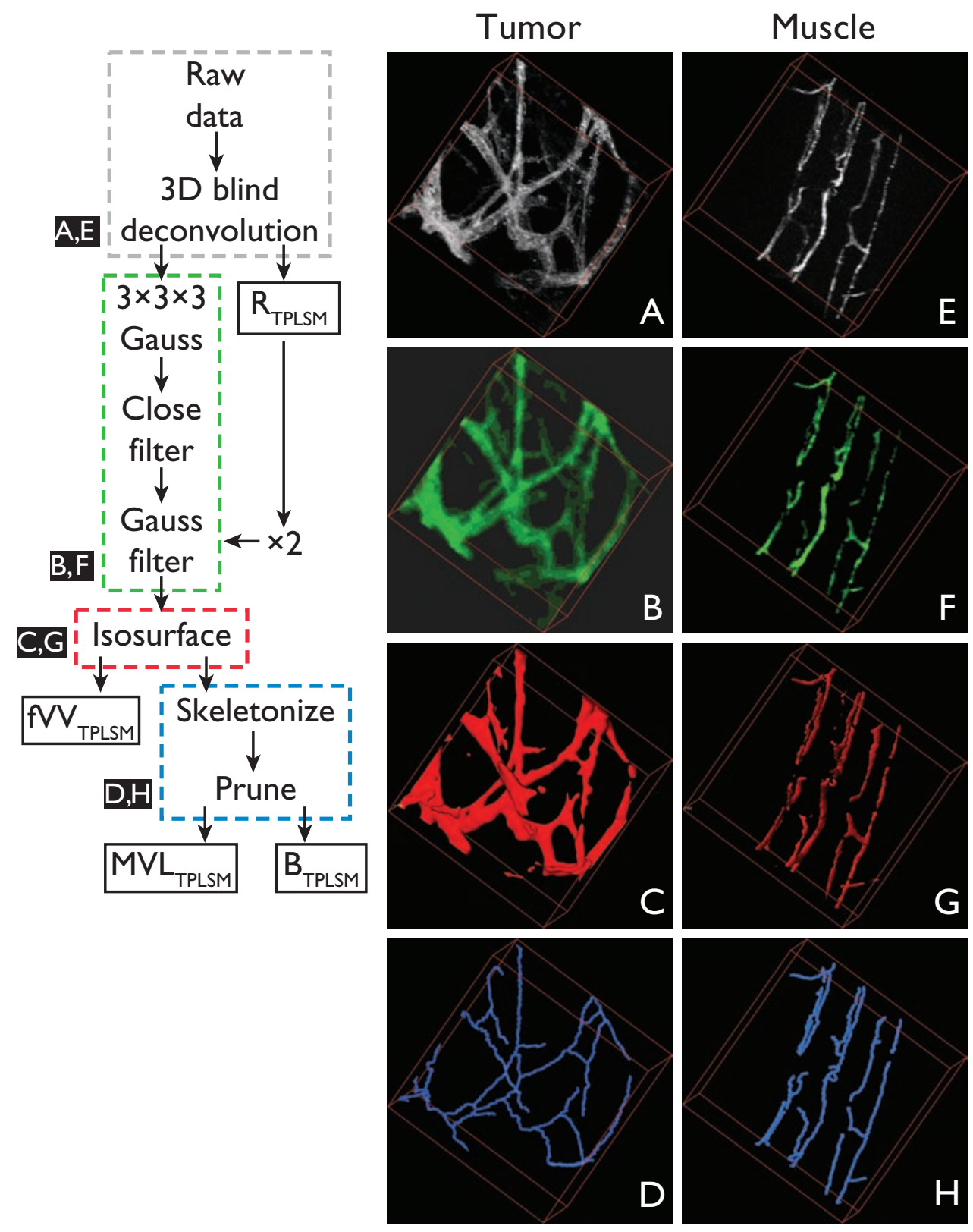

Figure 6.1: Schematic representation of the TPLSM data processing steps (left) with corresponding images for representative tumor (panels A-D, box size: $179 \times 179 \times 77 \mu \mathrm{m}^{3}$ ) and muscle (panels $\mathrm{E}-\mathrm{H}$, box size $179 \times 179 \times 52 \mu \mathrm{m}^{3}$ ) datasets. The different post-processing steps are indicated in different colors. Grey: data after 3D blind deconvolution (A/E). The median vessel radius $\mathrm{R}_{\text {TPLSM }}$ was measured during this step. Green: data after application of a $3 \times 3 \times 3$ Gauss filter, a closing filter, and a second Gauss filter $(B / F)$. Red: data after isosurface rendering, which was used to determine the fractional vessel volume $\mathrm{fVV}_{T P L S M}(C / G)$. Blue: skeletonized data $(D / H)$ used to extract the total microvessel length $M V L_{T P L S M}$ and degree of branching BTPLSM. 


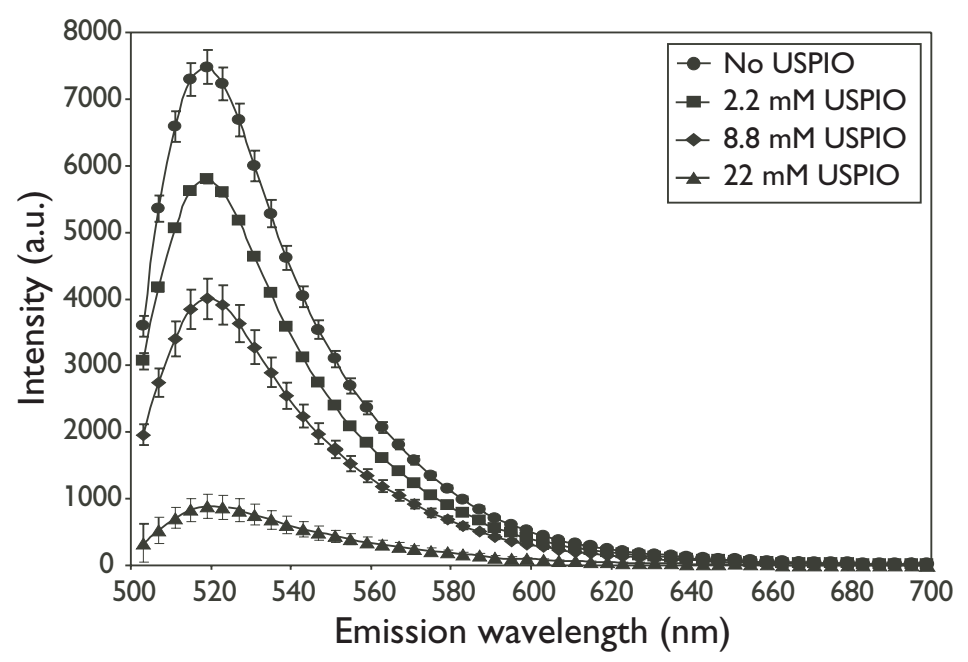

Figure 6.2: Negative effect of USPIOs on the fluorescence intensity of $\alpha$ CD31-FITC. A clearly decreasing signal intensity is observed in the emission spectrum of $\alpha$ CD31-FITC $(1 \mu \mathrm{M})$ with increasing USPIO concentration. The concentrations of $\alpha$ CD31-FITC and USPIO in the tissue were estimated to be approximately $0.7 \mu \mathrm{M}$ and $5 \mathrm{mM}$, respectively. Values are presented as mean \pm standard deviation.

\section{Statistical analysis}

Statistical analysis was performed using SPSS 15.0 (SPSS, Chicago, III). MRI data were tested using a paired non-parametric Wilcoxon signed-rank test. For TPLSM, the datasets from the tumor rim and core originated from different mice. Therefore, these data were analyzed using a non-paired non-parametric Mann-Whitney U-test.

\subsection{Results}

\section{Spectrophotometry}

Spectrophotometry revealed no inherent fluorescent signal of the USPIOs. However, the fluorescence intensity of $\alpha$ CD31-FITC decreased with increasing USPIO concentrations (Figure 6.2). Fluorescence lifetime imaging displayed no alterations in $\alpha$ CD31-FITC lifetime (not shown), indicating that fluorescence resonance energy transfer was absent. The low intensity of $\alpha \mathrm{CD} 31-\mathrm{FITC}$ was probably caused by scattering/absorption of $\alpha$ CD31-FITC fluorescence and/or absorption of excitation light by the USPIOs. 
Table 6.1: Overview of the mean pre- and post-contrast $R_{2}$ and $R_{2}^{*}$ values for tumor rim, tumor core, and muscle. The differences $\Delta R_{2}$ and $\Delta R_{2}^{*}$, and ADC values that were used for calculating the MR-derived vessel radius index and fractional blood volume are also provided. Averages were derived by first calculating the average per mouse from the pixel-based parameter maps (significantly enhanced voxels only). Next, values were averaged over the animals. Values are presented as median with their quartile ranges indicated in parentheses.

\begin{tabular}{lccc}
\hline & Tumor rim & Tumor core & Muscle \\
$R_{2, \text { pre }}\left(\mathrm{s}^{-1}\right)$ & $24.6(23.6-26.1)$ & $22.4(21.0-24.6)$ & $47.9(44.4-51.1)$ \\
$R_{2, \text { post }}\left(\mathrm{s}^{-1}\right)$ & $26.4(25.5-27.6)$ & $23.7(22.2-25.1)$ & $55.9(52.4-59.0)$ \\
$\Delta R_{2}\left(\mathrm{~s}^{-1}\right)$ & $1.7(1.2-2.0)$ & $1.1(1.1-1.4)$ & $8.0(6.5-9.4)$ \\
$R_{2, \text { pre }}^{*}\left(\mathrm{~s}^{-1}\right)$ & $175.5(144.4-199.2)$ & $138.4(104.4-140.9)$ & $145.1(119.2-164.8)$ \\
$R_{2, \text { post }}\left(\mathrm{s}^{-1}\right)$ & $255.4(211.4-314.5)$ & $193.8(158.9-231.4)$ & $220.9(158.8-246.0)$ \\
$\Delta R_{2}^{*}\left(\mathrm{~s}^{-1}\right)$ & $65.5(51.4-74.6)$ & $46.1(37.4-68.8)$ & $56.0(43.9-75.5)$ \\
$\mathrm{ADC}\left(\mu \mathrm{m}^{2} \mathrm{~s}^{-1}\right)$ & $1175(1048-1310)$ & $1212(1116-1355)$ & $1954(1729-2036)$ \\
\hline
\end{tabular}

\section{Animal inclusion}

Eight out of ten mice successfully developed a subcutaneous tumor. Therefore, tumor MR-VSI was performed in 8 mice, whereas muscle imaging was performed in 10. For TPLSM, a separate group of 11 tumor-bearing mice was used since a low and discontinuous fluorescent signal of the vasculature was observed in tissue of mice injected with USPIOs (data not shown). The total number of 3D TPLSM datasets for the tumor rim, tumor core, and muscle tissue was 10 (5 mice), 13 (6 mice), and 32 (9 mice), respectively.

\section{MR-VSI vascular morphology}

Average pre- and post-contrast $R_{2}$ and $R_{2}^{*}$ values, the differences $\Delta R_{2}$ and $\Delta R_{2}^{*}$, and the ADC values that were used for the MR-VSI calculations are summarized in Table 6.1. Figure 6.3 shows $T_{2}$-weighted anatomical images of a tumor with colorcoded overlay of the calculated vessel radius index $R I_{M R I}$ and fractional blood volume $f B V_{M R I}$. A heterogeneous distribution of $R I_{M R I}$ and $f B V_{M R I}$ was found throughout the tumor, with both parameters showing higher values in the tumor rim than in the tumor core (Figure $6.3 \mathrm{~A} / \mathrm{C}$ ). In healthy muscle tissue, the distribution was more homogeneous and significantly lower values of $R I_{M R I}$ and $f B V_{M R I}$ values were found compared with tumor tissue (Figure 6.3 B/D).

The differences in $\mathrm{RI}_{\mathrm{MRI}}$ and $\mathrm{fBV} \mathrm{MRI}_{\mathrm{M}}$ between tumor rim, tumor core, and muscle tissue were further investigated by histogram analysis (Figure 6.4), which confirmed a wide distribution for the tumor and a narrow distribution centered at relatively low values for muscle (Table 6.2). $\mathrm{RI}_{\mathrm{MRI}}$ values in tumor rim and tumor core were approximately 3-fold higher compared with muscle tissue. Although $\mathrm{RI}_{\mathrm{MRI}}$ was slightly 

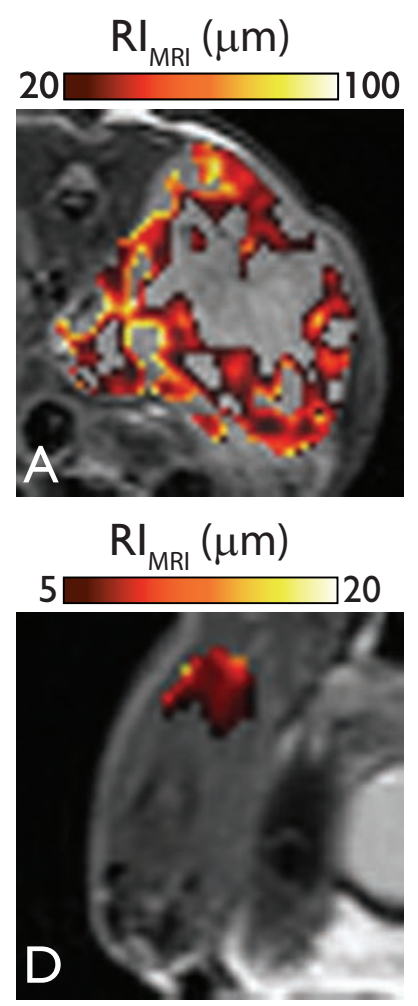
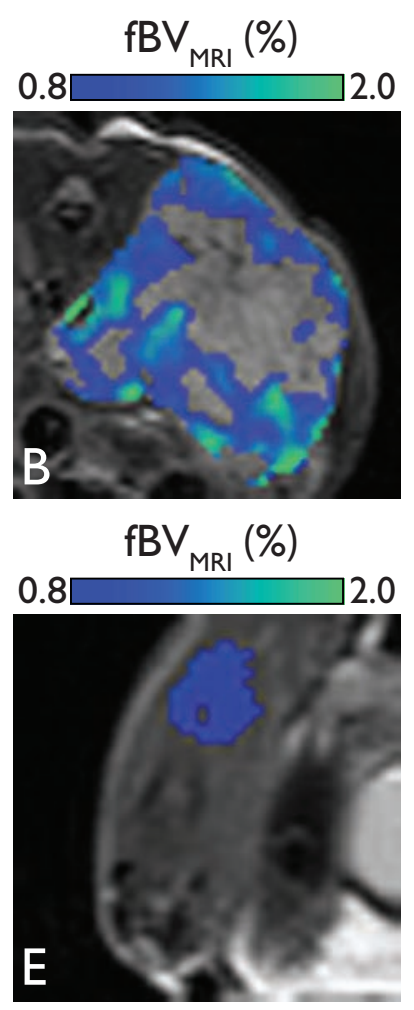
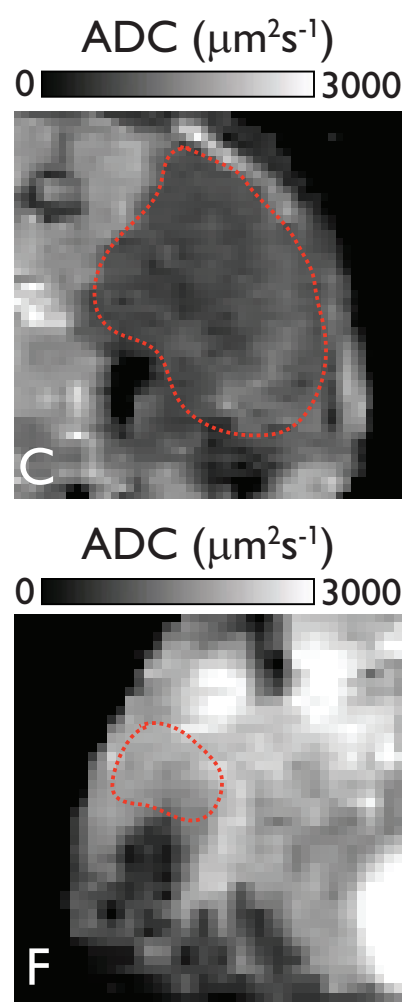

Figure 6.3: Sections of $T_{2}$-weighted images (TE $=30 \mathrm{~ms}$ ) of tumor $(A-C)$ and muscle (D-F) with color-coded overlay of $\mathrm{RI}_{\mathrm{MRI}}(\mathrm{A} / \mathrm{D})$ and $\mathrm{fBV} \mathrm{MRI}_{\mathrm{MI}}(\mathrm{B} / \mathrm{E})$. Note the different scales for $\mathrm{RI}_{\mathrm{MRI}}$ for tumor and muscle tissue. Panels $\mathrm{C}$ and $\mathrm{F}$ show representative $\mathrm{ADC}$ maps for tumor and muscle tissue. For clarity, the tumor and muscle regions are outlined by the dashed red lines. Note that the field-of-view is somewhat different for the ADC maps compared with the other images due to the differences in resolution in the slice direction.

higher in the tumor rim than in the tumor core, this difference was not significant. In contrast, $f B V_{M R I}$ was significantly higher in tumor rim compared with core, and in tumor rim compared with muscle (Table 6.2).

\section{TPLSM vascular morphology}

Qualitative comparison of the tumor and muscle tissue datasets showed clear differences in vessel radius and orientation (Figure 6.1). In muscle tissue, vessels were generally oriented parallel with the muscle fibers, with occasional interconnecting side branches, whereas the tumor microvasculature lacked a distinct orientation and displayed a more arbitrary, maze-like architecture. In correspondence with MR-VSI data, tumor tissue displayed a wide distribution in vessel radius (Figure 6.5). 

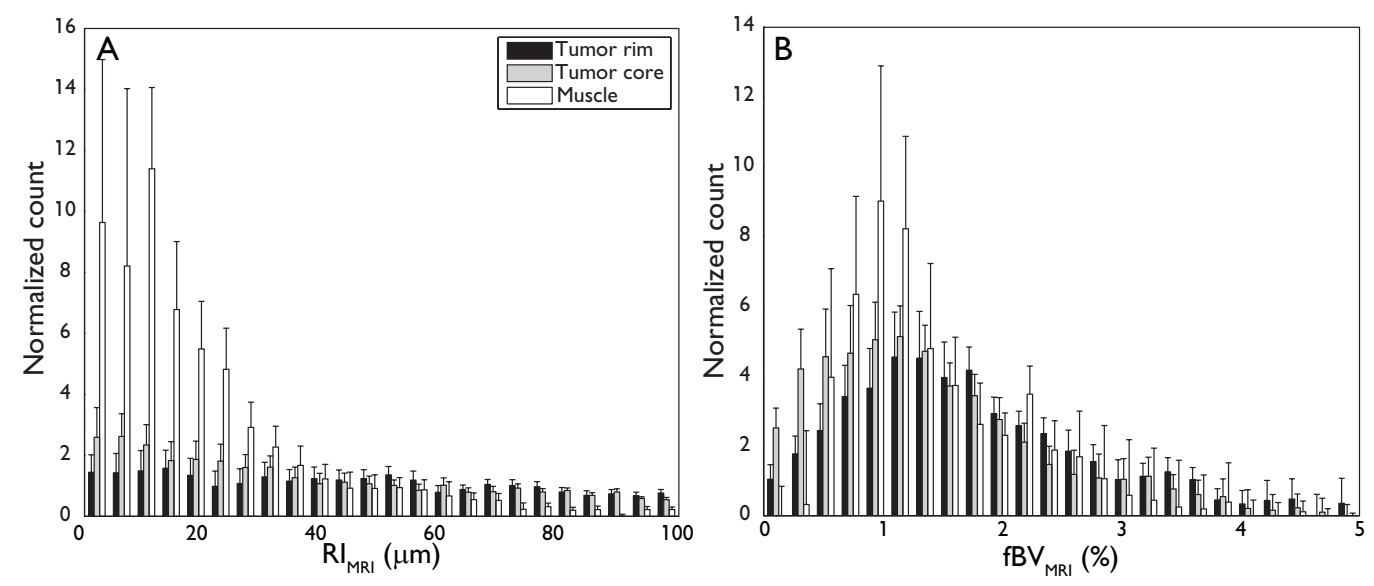

Figure 6.4: Histograms of the vessel radius index (A) and fractional blood volume (B) for the tumor rim (black), tumor core (grey) and muscle tissue (white), as determined using MR-VSI. Values were normalized to tissue volume and are represented as median \pm standard error.

$\mathrm{R}_{\text {TPLSM }}$ was significantly larger in the tumor rim and core compared to muscle tissue (Table 6.2). Similar to MR-VSI, no significant difference was observed in RTPLSM between tumor rim and core. However, the histogram of microvessel radius displayed a slight left-shift in RTPLSM in the tumor core compared to the rim, suggesting that the tumor core holds smaller sized microvessels (Figure 6.5). In accordance, the tumor rim displayed a significantly larger $\mathrm{V} V \mathrm{~V}_{\text {TPLSM }}$ compared with the tumor core and muscle tissue (Table 6.2). Moreover, the fVV $\mathrm{TPLSM}_{\text {in }}$ the tumor core was significantly larger than in muscle tissue. In addition, significantly higher $M V L_{T P L S M}$ and $B_{T P L S M}$ values were found in the tumor rim and core compared with muscle tissue.

\subsection{Discussion}

\section{Current findings}

In the present study, the vessel radius index and fractional blood volume, as determined by MR-VSI in subcutaneous tumors, were evaluated using 3D TPLSM. In addition, the performance of MR-VSI was investigated in healthy muscle tissue, which, in contrast to tumors, has a fully matured and highly organized microvasculature. Image processing of the TPLSM datasets resulted in values for the vessel radius and the fractional vessel volume that comply with literature $[78,213]$ (cf. Tables 6.2 and 6.3). MR-VSI displayed non-physiological vessel radius values for both the tumor and muscle tissue, thereby indicating that MR-VSI provides an index, and not an absolute 
Table 6.2: Overview of microvascular morphology parameters for blood vessels in the tumor rim, tumor core, and muscle tissue, as determined by MR-VSI and TPLSM. All values are presented as median with their quartile ranges indicated in parentheses. Note that no MRI equivalent is available for the total microvessel length MVL TPLSM and the degree of vessel branching BTPLSM.

\begin{tabular}{lccc}
\hline & \multicolumn{1}{c}{ Tumor rim } & Tumor core & Muscle \\
$\mathrm{RI}_{\text {MRI }}(\mu \mathrm{m})^{\mathrm{a}}$ & $38.0(33.6-49.9)^{\mathrm{b}}$ & $34.3(22.8-47.8)^{\mathrm{b}}$ & $11.9(7.0-16.7)$ \\
$\mathrm{R}_{\text {TPLSM }}(\mu \mathrm{m})$ & $6.0(4.5-6.6)^{\mathrm{b}}$ & $4.3(3.1-4.8)^{\mathrm{b}}$ & $2.2(1.7-2.7)$ \\
$\mathrm{fBV}$ MRI $(\%)$ & $1.8(1.2-2.2)^{\mathrm{b}, \mathrm{c}}$ & $1.2(0.9-1.7)$ & $1.2(0.9-1.5)$ \\
$\mathrm{fV}_{\text {TPLSM }}(\%)$ & $8.9(6.1-11.8)^{\mathrm{b}, \mathrm{c}}$ & $4.6(4.0-6.5)^{\mathrm{b}}$ & $1.5(1.0-1.9)$ \\
$\mathrm{MVL}{ }_{\text {TPLSM }}\left(\times 10^{-4} \mu \mathrm{m}^{-2}\right)$ & $8.1(5.2-11.4)^{\mathrm{b}}$ & $7.3(6.3-9.4)^{\mathrm{b}}$ & $3.5(2.7-5.3)$ \\
$\mathrm{B}_{\text {TPLSM }}\left(\times 10^{-6} \mu \mathrm{m}^{-3}\right)$ & $7.9(3.5-13.8)^{\mathrm{b}}$ & $11.0(9.8-14.9)^{\mathrm{b}}$ & $3.3(1.6-5.1)$ \\
\hline
\end{tabular}

a $\mathrm{RI}_{\mathrm{MRI}}$ : vessel radius index as determined by MR-VSI. RTPLSM: vessel radius as determined

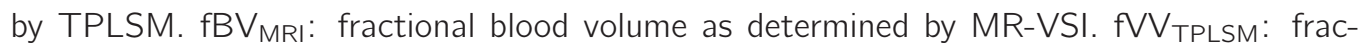
tional vessel volume as determined by TPLSM. MVLTPLSM: total microvessel length as determined by TPLSM. B BPLSM: degree of branching as determined by TPLSM. ${ }^{\mathrm{b}} \mathrm{P}<0.05$ compared with muscle tissue. ${ }^{c} P<0.05$ compared with tumor core.

measure, of the vessel radius. However, TPLSM and MR-VSI showed similar trends for the vessel radius (index) and the fractional blood volume in the observed tissues, i.e. generally higher values in the strongly vascularized tumor rim compared with the tumor core and muscle tissue.

The MR-derived vessel radius index was significantly higher in the tumor compared with muscle tissue, with no significant difference between tumor rim and core. The latter may be due to the heterogeneous distribution of vessel radius index found over the tumor. The fractional blood volume obtained with MR-VSI indicated that the tumor rim was more vascularized than the tumor core and muscle tissue. This corresponds with the reported higher level of angiogenic activity in the tumor rim $[113,119,158]$ and with more necrosis and higher interstitial pressures in the core.

With TPLSM, a heterogeneous distribution of vessel radius was observed over the tumor, showing higher values in the tumor rim than in the core. However, these differences were not statistically significant. Muscle tissue displayed a significantly smaller radius, which was approximately 2-3 fold smaller compared with the tumor (Table 6.2). The TPLSM-derived fractional vessel volume was significantly higher in the tumor rim compared with tumor core and muscle tissue, and in the tumor core compared with muscle tissue. The total microvessel length and the degree of vessel branching, which can be uniquely assessed by TPLSM, were also significantly higher in the tumor than in muscle tissue. Although the individual tumor vessels may appear shorter than the muscle vessels (Figure 6.1), note that TPLSM measures the total, and not the average, microvessel length. Taken together, these results indicate that the tumors were more strongly vascularized than skeletal muscle tissue. 


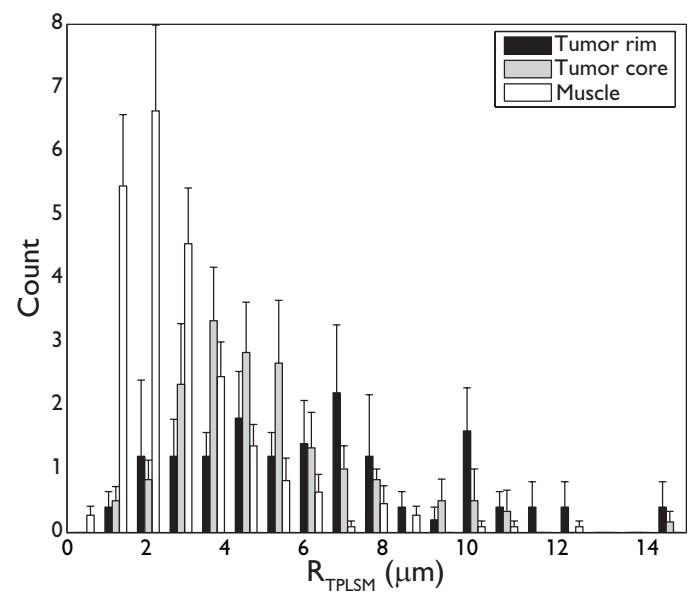

Figure 6.5: Histogram of the vessel radius as determined with TPLSM for the tumor rim (black), tumor core (grey), and muscle tissue (white). Values are presented as mean \pm standard error. Due to the limited number of available data points for $\mathrm{PV} \mathrm{V}_{\mathrm{TPLSM}}, \mathrm{MVL} \mathrm{LPLSM}_{\mathrm{T}}$, and $B_{\text {TPLSM }}$ ( 1 per animal versus 8 for $R_{\text {TPLSM }}$ ), histogram analysis was impractical for these parameters.

In comparison with TPLSM, MR-VSI showed a 6-8 fold overestimation of the vessel radius index for all tissue types. This may be explained by partial volume effects due to the limited spatial resolution of MR-VSI, relative to the dimensions of the microvessels in tumor and muscle tissue. In addition, Kiselev et al. suggested that an overestimation of vessel caliber can be explained by a deviation from the static dephasing regime of relaxation around capillaries, and by the native paramagnetism of venous blood, which are both neglected in the simplified MR-VSI theory [60]. Interestingly, lower intratumoral blood volumes were found for MR-VSI than for TPLSM, whereas these values for the skeletal muscle were similar. These observations may be related to the heterogeneous perfusion effects observed in tumors [63]. Hence, tumor regions with no or relatively low USPIO concentration will result in a lower detected blood volume. This heterogeneous perfusion may also have contributed to the exclusion of approximately $40 \%$ of the tumor voxels in the VSI calculations (see Section 6.2). In addition, whereas in vivo injection of USPIOs results in labeling of perfused, i.e., functional, microvasculature only, ex vivo fluorescent staining of vascular endothelium with $\alpha$ CD31-FITC results in labeling of both perfused and non-perfused microvasculature. This theoretically may have contributed to an over-

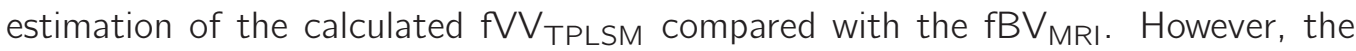
good correspondence of the present TPLSM results with literature values $[78,213]$ suggests that these differences in vascular labeling did not contribute to the observed discrepancies between MR-VSI and TPLSM.

In the present study, a literature value of $0.571 \mathrm{ppm}$ measured at a field strength of $2.35 \mathrm{~T}$ [58] was used for the susceptibility $\Delta \chi$. However, the susceptibility may vary between USPIO batches and as a function of external field strength. At the 
currently applied magnetic field of $7 \mathrm{~T}$ the susceptibility is expected to be somewhat lower. Although this would reduce the underestimation of relative blood volume, it results in an even larger overestimation of vessel radius. Given that the susceptibility is a constant in the definitions of vessel size index and relative blood volume, any error in susceptibility will affect the results in different tissue regions equally. In addition, the same USPIO batch was used for all experiments. Consequently, the relative differences between tumor rim, tumor core and muscle will not depend on the absolute susceptibility value, which justifies the use of a reported reference value.

Although the MR vessel radius index and fractional blood volumes did not correspond with their TPLSM equivalents, the observed differences between tumor rim, tumor core, and muscle showed the same trends for both methods. MR-VSI is therefore not suited for absolute quantification of vascular morphology, but does allow detection of differences within tumors and between tissues. This suggest that MRVSI may be applied for longitudinal in vivo evaluation of relative changes in vessel architecture, induced for instance by tumor growth or by anti-angiogenic therapy.

\section{Quantitative vascular morphology in current literature}

Table 6.3 provides an overview of available literature values for tumor vessel radius (index) and blood volume, measured either by MR-VSI, histology, or microscopy techniques, and the applied experimental setup. Direct comparison with the present results is hampered due to large differences in tumor models. Different tumor locations, stages of tumor growth, and animal species have been studied and a distinction between the tumor rim and core is usually not made. Moreover, tumor vasculature typically harbors unpredictable flow, adding non-systematic variability to MR-VSI measurements as these were shown to depend on the intravascular USPIO concentration [214]. Also, $\Delta R_{2}$ and $\Delta R_{2}^{*}$ measurements depend on technical parameters such as field strength, pulse sequence, field homogeneity and shimming, echo times, etc.

Extensive data processing is required to convert contrast agent induced changes in signal intensity into quantitative vessel parameters, and different approaches have been described. For instance, different maximum $\mathrm{RI} \mathrm{MRI}_{\mathrm{I}}$ values were applied above which vessel radius was considered unrealistic: an upper limit of $100 \mu \mathrm{m}$ was set by Troprès et al. [58] whereas Valable et al. used a $50 \mu \mathrm{m}$ threshold [201]. In addition, diffusion-weighted experiments are not always conducted, although the measurement of individual ADC values was shown to be important for accurate MR-VSI [60].

Taken together, reported literature values are highly variable, indicating that MRVSI is strongly dependent on experimental setup (Table 6.3). Also, vessel radius and diameter appear to be used interchangeably, thereby adding a factor 2 to the observed variability. 
Table 6.3: Overview of available literature values for the average tumor vessel radius (index) R and the fractional blood voume fBV, measured by MR-VSI, histology, or intravital microscopy. The applied tumor model, tumor location, animal kind, and contrast agent are also presented.

The contrast agent dose was $200 \mu \mathrm{mol} \mathrm{Fe/kg} \mathrm{body} \mathrm{weight,} \mathrm{unless} \mathrm{indicated} \mathrm{otherwise.} \mathrm{Values} \mathrm{are} \mathrm{presented} \mathrm{as} \mathrm{mean} \pm$ standard deviation.

\begin{tabular}{|c|c|c|c|c|c|c|c|}
\hline Tumor model & Location & Animal & Contrast agent ${ }^{a}$ & $\mathbf{R}(\mu \mathbf{m})$ & fBV $(\%)$ & Technique $^{b}$ & Ref \\
\hline \multirow[t]{5}{*}{ C6 glioma } & \multirow[t]{5}{*}{ Brain } & \multirow[t]{5}{*}{ Rat } & MION & $12.5 \pm 6.8$ & $N A^{c}$ & MR-VSI & {$[56]$} \\
\hline & & & \multirow[t]{2}{*}{ Sinerem ${ }^{\circledR}$} & $20.0 \pm 6.3$ & NA & MR-VSI & [59] \\
\hline & & & & $5.8 \pm 4.1$ & $2.0 \pm 0.7$ & Histo & \\
\hline & & & \multirow[t]{2}{*}{ Sinerem ${ }^{\circledR}$} & $19.5 \pm 4.8$ & $4.0 \pm 1.4$ & MR-VSI & \multirow[t]{2}{*}{ [201] } \\
\hline & & & & $9.9 \pm 2.1$ & $2.9 \pm 0.6$ & Histo & \\
\hline \multirow[t]{2}{*}{ RG2 glioma } & \multirow[t]{2}{*}{ Brain } & \multirow[t]{2}{*}{ Rat } & \multirow[t]{2}{*}{ Sinerem ${ }^{\circledR}$} & $11.0 \pm 2.0$ & $5.2 \pm 1.6$ & MR-VSI & \multirow[t]{2}{*}{ [201] } \\
\hline & & & & $7.4 \pm 1.3$ & NA & Histo & \\
\hline $\begin{array}{l}\mathrm{GH} 3 \\
\text { prolactinoma }\end{array}$ & S.C $C^{d}$ & Rat & Sinerem $^{\circledR}$ & $13 \pm 4$ & $2.9 \pm 0.8$ & MR-VSI & [202] \\
\hline $\begin{array}{l}\text { Shionogi } \\
\text { prostate } \\
\text { carcinoma }\end{array}$ & S.c. & Mouse & Sinerem ${ }^{\circledR}$ & $35.2 \pm 25.5$ & NA & MR-VSI & {$[62]$} \\
\hline B16 melanoma & S.C. & Mouse & $\begin{array}{c}\text { Clariscan } \\
45 \mu \mathrm{mol} \mathrm{Fe} / \mathrm{kg}\end{array}$ & $13.5 \pm 1$ & $4.1 \pm 0.5$ & MR-VSI & [61] \\
\hline $\begin{array}{l}\text { LS174T } \\
\text { colorectal } \\
\text { adenocarcinoma }\end{array}$ & $\begin{array}{l}\text { Skin-fold } \\
\text { chamber }\end{array}$ & Mouse & $\begin{array}{l}\text { Texas-Red } \\
\text { labeled bovine } \\
\text { serum albumin } \\
\text { and FITC-dextran }\end{array}$ & $6.1 \pm 0.5^{\mathrm{e}}$ & $9.2 \pm 2.9$ & IVM & {$[78,213$} \\
\hline \multirow{2}{*}{$\begin{array}{l}\text { HaCaT-ras-A- } \\
\text { 5RT3 skin } \\
\text { squamous cell } \\
\text { carcinoma }\end{array}$} & \multirow[t]{2}{*}{ S.C. } & \multirow[t]{2}{*}{ Mouse } & \multirow[t]{2}{*}{$\begin{array}{l}\text { VSOP C200 } \\
\text { (Ferropharm) }\end{array}$} & $62 \pm 18$ & NA & MR-VSI & [203] \\
\hline & & & & $10 \pm 7^{\mathrm{e}}$ & $1.4 \pm 0.2$ & Histo & \\
\hline \multirow{2}{*}{$\begin{array}{l}\text { A431 skin } \\
\text { squamous cell } \\
\text { carcinoma }\end{array}$} & \multirow[t]{2}{*}{ S.C. } & \multirow[t]{2}{*}{ Mouse } & \multirow[t]{2}{*}{$\begin{array}{l}\text { VSOP C200 } \\
\text { (Ferropharm) }\end{array}$} & $42 \pm 7$ & NA & MR-VSI & [203] \\
\hline & & & & $10 \pm 3^{e}$ & $1.1 \pm 0.3$ & Histo & \\
\hline Various & Brain & Human & $\begin{array}{l}\text { Magnevist }^{\circledR} \\
0.2 \mathrm{mmol} / \mathrm{kg}\end{array}$ & $79 \pm 68$ & NA & MR-VSI & [60] \\
\hline
\end{tabular}

${ }^{a}$ MION: Monocrystalline iron oxide nanoparticle; VSOP: Very small superparamagnetic iron oxide particle. ${ }^{\text {b MR- }}$ VSI: Magnetic resonance vessel size imaging; Histo: Histology; IVM: Intravital microscopy. ${ }^{c}$ Not available. ${ }^{\text {dS.c.: }}$ subcutaneous. ${ }^{\mathrm{e}}$ Calculated from reported vessel diameters. 


\section{Limitations}

Direct comparison of the MR-VSI results with 3D TPLSM in the same tumors was unfeasible in the present study due to a reduction in $\alpha$ CD31-FITC intensity by USPIOs. This necessitated the use of two separate animal groups for MR-VSI and TPLSM. Moreover, tissue freezing and thawing may have caused deformation and hence affect TPLSM quantification of microvascular morphology. These problems may be circumvented by applying long-circulating bimodal, i.e. fluorescent and superparamagnetic, nanoparticles [215]. Combined with in vivo TPLSM [212], this would theoretically provide the optimal validation method for MR-VSI. Nevertheless, the current ex vivo TPLSM results are in good agreement with previous in vivo studies $[78,213]$.

During TPLSM post-processing, blind 3D deconvolution was performed iteratively using an initial point spread function based on the configuration of the optical system. Although the validity of this approach was confirmed using fluorescent microspheres, Von Tiedemann et al. showed that significant improvements may be obtained when using an a depth-dependent point spread function that is automatically estimated from the dataset [216]. However, this method is still under development.

\section{Conclusions}

MR-VSI allows in vivo estimation of the microvascular radius index and blood volume in both tumor and healthy muscle tissue. TPLSM offers high-resolution 3D visualization of the microvasculature and provides a useful tool in preclinical research. Compared with 3D TPLSM, MR-VSI resulted in a large overestimation of the average vessel radius index, whereas the fractional blood volume was slightly lower. However, for both techniques, morphologic measures were higher for tumor than for muscle tissue, and higher for tumor rim relative to core. Thus, although MR-VSI is not suitable for absolute quantification of vessel radius and blood volume, the image contrast of MR-VSI reflects microvascular morphology and spatial heterogeneity thereof. MR-VSI may therefore be suitable to investigate relative microvascular differences between tissues and the effects of anti-angiogenic therapy in longitudinal studies.

\section{Acknowledgements}

We thank Guerbet for kindly providing Sinerem ${ }^{\circledR}$. We thank the following people for their valuable contributions to this study: Ludwig Dubois for in vitro growth of the LS174T tumor cells, Niek van Stipdonk for technical assistance during animal preparations, Wim Engels for assistance with TPLSM data analysis, and Jonathan Girroir and Yuri Gaidoukevitch from MediaCybernetics for valuable discussions on deconvolution and quantification of the TPLSM data. 



\section{Part II}

\section{Myocardial microvasculature}





\section{Molecular MRI of cardiac angiogenesis after acute myocardial infarction}

Angiogenesis is a natural mechanism to restore perfusion to the ischemic myocardium after acute myocardial infarction (MI). Presently, therapeutic angiogenesis is being explored as a novel treatment for MI patients. However, sensitive, non-invasive in vivo measures of therapeutic efficacy are currently lacking and need to be developed. Here, a molecular magnetic resonance imaging (MRI) method is presented to non-invasively image angiogenic activity in vivo in a murine model of MI using cyclic Asn-Gly-Arg (cNGR)-labeled paramagnetic quantum dots (pQDs). The tripeptide cNGR homes specifically to CD13, an aminopeptidase that is strongly upregulated during myocardial angiogenesis. Intravenous administration of cNGR-pQDs resulted in a strong negative contrast that was mainly located in the infarcted myocardium. This negative contrast was significantly less in MI-mice injected with unlabeled pQDs, and in sham-operated mice injected with cNGR-pQDs. Validation with ex vivo two-photon laser scanning microscopy (TPLSM) revealed a strong colocalization of cNGR-pQDs with vascular endothelial cells, whereas unlabeled pQDs were mostly extravasated and diffused through the tissue. Additionally, TPLSM demonstrated significant microvascular remodeling in the infarct/border zones compared with remote myocardium. In conclusion, data showed that cNGR-pQDs allow selective, non-invasive detection of angiogenic activity in the infarcted heart using in vivo molecular MRI and ex vivo TPLSM.

Circulation. 2009, provisionally accepted 


\subsection{Introduction}

Neovascularization through angiogenesis [2] provides a natural repair mechanism to restore perfusion of ischemic tissue after myocardial infarction (MI), and is a determinant of post-infarct remodeling and patient prognosis $[5,217]$. Consequently, stimulation of the angiogenic response, e.g. via administration of growth factor genes or proteins, seems an attractive option to reduce ischemic injury in the myocardium after MI [218-222], especially in patients where traditional revascularization failed. Although promising results were obtained with therapeutic angiogenesis in animal models of $\mathrm{MI}$, double-blind placebo-controlled clinical trials have been disappointing [218-222]. These contradictory outcomes may be related to the choice of therapeutic agent, route of administration, selection of clinical trial populations, and the absence of a sensitive, validated in vivo (surrogate) marker of therapeutic efficacy $[221,222]$.

Read-out tools that are able to evaluate the efficacy of angiogenic therapy should be non-invasive, repeatable, and sensitive to early therapeutic responses. Traditional non-invasive imaging techniques such as nuclear imaging, echocardiography or magnetic resonance imaging (MRI), detect the physiological consequences of angiogenesis, i.e. improved myocardial perfusion or global cardiac function, which occur relatively late during the angiogenic process. Their applicability to evaluate myocardial angiogenesis therefore remains to be further investigated [221]. In contrast, molecular imaging potentially permits early detection of myocardial angiogenesis, i.e., before changes in perfusion and/or function become apparent, via specific detection of upregulated disease markers using targeted contrast agents $[108,172]$. Currently, only few reports are available that describe molecular imaging of cardiac angiogenesis using nuclear $[223,224]$ or optical [143] imaging.

Here, we investigated the applicability of molecular MRI to detect angiogenic activity non-invasively in vivo in the mouse heart after induction of acute MI. Clinical advantages of MRI include excellent soft tissue contrast and spatial resolution, and absence of ionizing radiation. However, compared with nuclear and optical imaging, the detection limit for molecular markers is significantly higher for MRI.

Previously developed Gd-labeled paramagnetic quantum dots ( $p Q D s)$ conjugated with the cyclic tripeptide Asn-Gly-Arg (cNGR, Chapter 3) were chosen as contrast agent to selectively image the myocardial neovasculature. cNGR homes specifically to CD13, a membrane-bound aminopeptidase that is strongly upregulated on endothelial cells of angiogenic vessels in the infarcted area and border zones of the myocardium, with a peak expression level at 7 days post MI [136, 140,141,143]. The present study for the first time shows the applicability of non-invasive molecular MRI to detect myocardial angiogenesis in vivo using a cNGR-labeled contrast agent. The specificity of the targeted agent was supported by a significantly lower image contrast in shamoperated mice, and in $\mathrm{Ml}$-mice receiving unlabeled control contrast agent. Results 
were confirmed using ex vivo 3D two-photon laser scanning microscopy (TPLSM), which provided information on microvascular structure and contrast agent localization at a subcellular resolution.

\subsection{Methods}

\section{Contrast agents}

Streptavidin coated CdSe-based quantum dots with $585 \mathrm{~nm}$ emission wavelength ( $1 \mu \mathrm{M}$ in borate buffer $\mathrm{pH}$ 8.3) were purchased from Invitrogen (Breda, The Netherlands). cNGR-labeled pQDs were prepared freshly for each experiment by mixing $100 \mu \mathrm{L}$ QDs with biotinylated Gd-wedge, a poly(lysine) dendrimer comprising 8 Gd-DTPA moieties, and biotinylated cNGR peptide in a molar ratio of 1:24:6, as described in Section 3.2. Biotinylated Gd-wedge and CNGR were synthesized using tBoc solid-phase peptide synthesis [147-149]. Unlabeled pQDs were prepared similarly, only without cNGR addition. The maximum number of cNGR ligands and Gd-DTPA groups per cNGR-pQD particle was 6 and 192, respectively (Chapter 3).

For TPLSM measurements, vascular endothelial cells were fluorescently labeled by incubating the hearts for 30 minutes in anti-CD31-FITC $(0.5 \mathrm{mg} / \mathrm{mL}$, BD Biosciences Pharmingen, Alphen a/d Rijn, the Netherlands), diluted 20x in Hanks balanced salt solution (HBSS) pH 7.4.

\section{Animal model}

All experiments were approved by the institutional ethics committee on animal welfare. Ml was induced in 12-weeks old male Swiss mice via permanent occlusion of the left anterior descending artery (LAD), as described previously [225]. In brief, $0.1 \mathrm{mmol} / \mathrm{kg}$ burprenorphin s.c. (Temgesic ${ }^{\circledR}$, Schering-Plough, Utrecht, The Netherlands) was given as pre-operative analgesia. Mice were anesthetized using 5\% isoflurane (Abbott Laboratories Ltd, Queensborough, UK) in medical air, intubated and ventilated with $1-2 \%$ isoflurane at 150 respirations per minute using a microventilator (UNO Roestvaststaal BV, Zevenaar, The Netherlands). The heart was exposed via lateral thoracotomy and the LAD was ligated just below the branch-point of the anterior interventricular descending and diagonal arteries using a 6-0 polypropylene thread (Surgipro II, Syneture, Tyco Healthcare, Gosport, UK). This ligation site produced transmural, anterolateral, apical infarctions with a good post-operative survival rate of approximately $80 \%$. The thorax was closed using 5-0 polypropylene and the skin was sutured using 5-0 silk. Animals recovered overnight at $30{ }^{\circ} \mathrm{C}$. Shamoperated mice underwent the same surgery without tying the ligature (thread was only pulled through the myocardium). MRI experiments were performed 7 days after surgery $[143,223]$. 
Prior to MRI, mice were anesthetized as described above and were allowed to breathe freely thereafter. The left jugular vein was exposed and contrast agent was injected intravenously using a 30 gauge needle. Closure of the vein was induced by applying pressure with an absorption triangle (Fine Science Tools, Heidelberg, Germany). Next, the wound was closed and the mouse was transferred to an animal bed with built-in anesthesia mask for MRI measurements. Neonatal ECG electrodes ( $3 \mathrm{M}$, St Paul, MN) were placed on the paws of the right front leg and left hind leg and connected to an MR compatible small animal monitoring system (SA Instruments, Stony Brook, NY). Temperature and respiratory rate were continuously monitored as well.

\section{MRI protocol}

MRI was performed on a $7 \mathrm{~T}$ Bruker Biospec 70/30 USR (Bruker Biospin GmbH, Ettlingen, Germany) using the BGA12-S mini-imaging gradient (maximum gradient strength $720 \mathrm{mTm}^{-1}$, slew rate $6000 \mathrm{Tm}^{-1} \mathrm{~s}^{-1}$ ), interfaced to an AVANCE II console. Images were acquired using a $3.5 \mathrm{~mm}$ inner diameter quadrature volume resonator. First, a bright blood cine image with 10 cardiac phases was recorded in horizontal long axis view using a retrospectively self-gated protocol (IntraGate ${ }^{T M}$, Bruker Biospin). Second, a short axis, apical self-gated cine image was recorded, passing through the infarction as seen on the long axis view (Figure 7.1). This short axis orientation was applied for all subsequent images. Next, ECG-triggered, respiratory gated, end-diastolic bright blood gradient echo images were recorded as follows: repetition time $15 \mathrm{~ms}$, echo time 2.9 or $6.0 \mathrm{~ms}$, flip angle $50^{\circ}, 1$ slice, $1 \mathrm{~mm}$ thickness, 4 signal averages, $4 \times 4 \mathrm{~cm}^{2}$ field-of-view, $256 \times 256$ matrix, in-plane resolution $0.16 \times 0.16 \mathrm{~mm}^{2}$, acquisition time approximately 3 minutes. Images were recorded up to 2 hours after contrast agent injection, after which mice were sacrificed by cervical dislocation. 15 minutes after death, mice were repositioned in the MRI scanner and postmortem short axis spin echo images were recorded (repetition time $1300 \mathrm{~ms}$, echo time $9.1 \mathrm{~ms}, 5$ slices, $1 \mathrm{~mm}$ thickness, $4 \times 4 \mathrm{~cm}^{2}$ field-of-view, $256 \times 256$ matrix), covering most of the left ventricle (LV). These images allowed more accurate infarct size determination than in vivo images, since the healthy myocardium significantly thickens postmortem, whereas the infarcted myocardium stays thin. Finally, hearts were excised, embedded in optimal cutting temperature (OCT) compound (Sakura Finetek Europe, Zoeterwoude, the Netherlands), snap-frozen in ice-cold 2-methylbutane (Acros Organics, Geel, Belgium) and transferred to liquid nitrogen. Tissues were stored at $-80{ }^{\circ} \mathrm{C}$ until TPLSM measurements.

\section{MRI analysis}

All data processing was performed in Matlab (The MathWorks, Natick, MA), unless stated otherwise. Regions of interest were drawn manually in MRIcro [152]. 

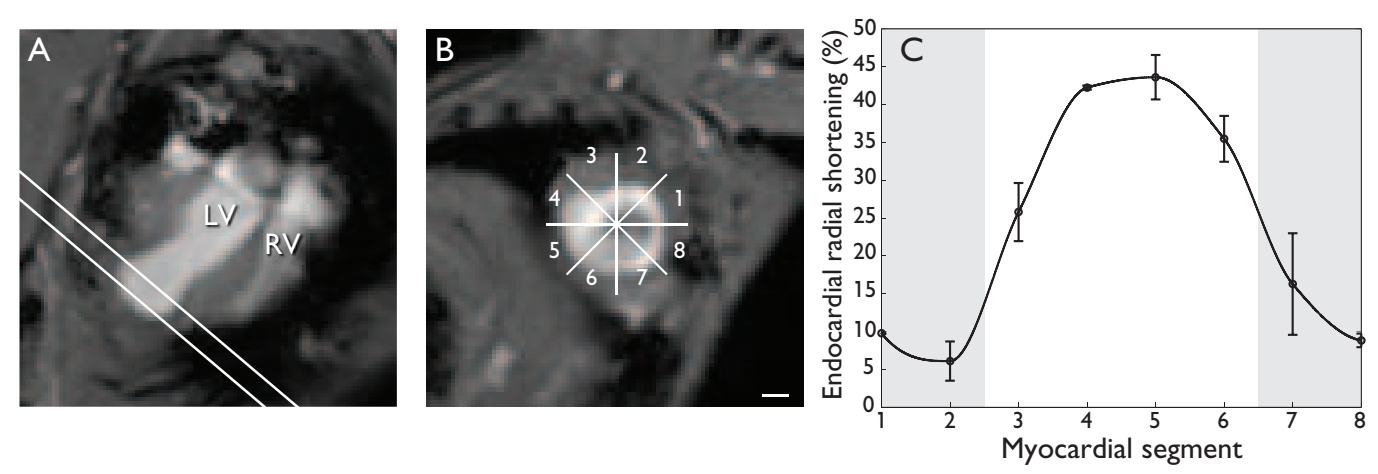

Figure 7.1: A) Horizontal long axis image showing the left (LV) and right (RV) ventricles of a mouse with MI. Diagonal lines indicate the location of the short axis image (slice thickness $1 \mathrm{~mm}$ ). Note that due to LV dilatation, the RV was usually not visible in the apical short axis images. B) Short axis image showing segmentation of the LV into 8 radial segments. Bar: $1 \mathrm{~mm}$. C) Endocardial radial shortening (ERS) versus myocardial segment. Segments $1,2,7$, and 8 showed a reduced ERS and were categorized as infarct/border zone (grey), whereas segments 3-6 were considered remote myocardium. The anterolateral location of the infarction corresponds with the territory that is normally perfused by the LAD.

\section{Ejection fraction}

Orthogonal long and short axis cine images were used to determine the LV ejection fraction (EF). Therefore, end-diastolic (ED) and end-systolic (ES) volumes were estimated using the biplane ellipsoid model [226] and the EF was calculated as (EDvolume - ESvolume)/EDvolume.100\%.

\section{Infarct size}

The infarct size was defined as the percentage of the LV surface that was infarcted and was determined as follows. The LV circumference was measured in each slice of the postmortem images using ImageJ [227] and multiplied by the slice thickness to generate the total LV surface area. The size of the infarcted area was measured analogously. Next, the infarct area was divided by the LV area and multiplied by $100 \%$ to obtain the infarct size.

\section{Myocardial segmentation}

To estimate local cardiac function, the LV myocardium was divided into 8 radial segments (Figure 7.1B, [223]). In each segment, the contractile function was assessed via the endocardial radial shortening (ERS) [228]. To this extent, endocardial contours were drawn on the ES and ED short axis cine frames and the radius $r$ was defined as the distance between the endocardial border and the LV center. The ERS was 
then calculated as $\left(r_{E D}-r_{E S}\right) / r_{E D} \cdot 100 \%$ and plotted as function of segment number (Figure 7.1C). Segments with reduced ERS were categorized as infarct/border zone by two readers in consensus. The remaining segments were considered remote myocardium. Myocardial segmentation was performed for each Ml-mouse individually as infarct size and location varied between animals. No segmentation was performed for sham-operated mice.

\section{Hypointense area}

The size of the hypointense area was measured in each segment by counting the number of voxels with signal intensity below a threshold value and multiplying this by the voxel size. Thresholds were defined for each image individually as the mean signal intensity in a reference region minus two times the standard deviation in this region. Reference regions were drawn manually outside the heart in non-angiogenic skeletal muscle tissue.

\section{TPLSM protocol}

Hearts were thawed and washed in HBSS to remove excess OCT. Next, hearts were incubated for 30 minutes in anti-CD31-FITC and embedded in 2\% (w/v) agarose gel (Invitrogen). Images were recorded in the infarction (identified visually based on the pale color of the MI region and location of the ligature), the border zone (0.5-1.0 mm proximal to the infarction), and remote myocardium (basal part of the heart).

In-depth TPLSM images were recorded with an Eclipse E600FN upright microscope (Nikon, Tokyo, Japan) and a Radiance 2100MP optical imaging system (Bio-Rad, Hemel Hempstead, UK), as described in Section 3.2. Fluorophores were excited by a mode-locked Tsunami Ti:Sapphire laser (Spectra-Physics, Mountain View, CA) with an $800 \mathrm{~nm}$ central wavelength and a $120 \mathrm{fs}$ pulse width. Datasets were acquired using a $60 \times$ water-dipping objective lens (Nikon) with a 1.00 numerical aperture. FITC (520 - 560 nm) and QD (570 - $600 \mathrm{~nm})$ fluorescence were detected by photomultiplier tubes (Electron Tubes, Ruislip, UK) and color-coded in green and red, respectively. An in-plane resolution of $0.35 \times 0.35 \mu \mathrm{m}^{2}$ was obtained using a $179 \times 179 \mu \mathrm{m}^{2}$ field-of-view and a $512 \times 512$ matrix. The pixel dwell time was $11.8 \mu \mathrm{s}$, which together with a two-fold Kalman averaging resulted in an acquisition rate of $0.16 \mathrm{~Hz}$ for each of the imaging planes in the 3D dataset. The interplanar distance was 1.05 or $0.6 \mu \mathrm{m}$.

\section{TPLSM analysis}

TPLSM images were analyzed semi-quantitatively using ImageJ. The following parameters related to microvascular structure and $\mathrm{PQD}$ distribution were considered: 
number of vessels, vessel size, vessel structure, number of pQDs, intravascular pQDs, colocalization of $\mathrm{pQDs}$ with endothelial cells, and $\mathrm{pQD}$ extravasation.

\section{Statistical analysis}

All values are presented as mean \pm standard error. Statistical analysis was performed in SPSS 17.0 (SPSS, Chicago, III). Independent and related samples were compared using a non-parametric Mann-Whitney U-test or Wilcoxon signed ranks test, respectively. $\mathrm{P} \leq 0.05$ was considered significant.

\subsection{Results}

\section{Left ventricular function and infarct size}

No significant differences in body weight, heart rate, and respiration rate were observed between MI-mice and sham-operated mice (Table 7.1). All MI-mice showed a strongly dilated LV on horizontal long axis cine images (Figure 7.1A). The infarcted myocardium was clearly visible as a hypo- or akinetic apical region with a thin myocardial wall. Short axis cine images were recorded perpendicular to the long axis plane and transected the $\mathrm{Ml}$ area (Figure 7.1B). Here, an impaired contractile function was also clearly observed. In contrast, sham-operated mice showed normal LV contractility and wall thickness on long and short axis cine images.

Global LV function was assessed by the EF, which was determined using the biplane ellipsoid model [226]. The validity of the model was confirmed by the EF of sham-operated mice (Table 7.1), which corresponded with previously published values for healthy mice [229-231]. In contrast, a significantly lower EF was found for both infarct groups (Table 7.1). No differences in EF were found between the MI groups, indicating similar global LV function. Also, the infarct size was not significantly different between the MI groups (Table 7.1).

Table 7.1: Summary of mouse body weight, heart rate, respiration rate, left ventricular ejection fraction, and infarct size 7 days after surgery. Heart and respiration rates were derived from the self-gated IntraGate ${ }^{T M}$ reconstruction algorithm.

\begin{tabular}{lllllll}
\hline Group & $\mathbf{n}$ & $\begin{array}{l}\text { Body } \\
\text { weight }\end{array}$ & $\begin{array}{l}\text { Heart rate } \\
\text { (bpm) }\end{array}$ & $\begin{array}{l}\text { Respiration } \\
\left.\text { rate } \mathbf{( m i n}^{-1}\right)\end{array}$ & $\begin{array}{l}\text { Ejection } \\
\text { fraction (\%) }\end{array}$ & $\begin{array}{l}\text { Infarct } \\
\text { size (\%) }\end{array}$ \\
Ml + cNGR-pQDs & 6 & $39.3 \pm 1.9$ & $437 \pm 29$ & $62 \pm 2$ & $38 \pm 3^{\mathrm{a}}$ & $32 \pm 5$ \\
$\mathrm{Ml}+$ unlabeled pQDs & 4 & $40.9 \pm 1.2$ & $408 \pm 4$ & $55 \pm 6$ & $42 \pm 5^{\mathrm{a}}$ & $29 \pm 5$ \\
Sham + cNGR-pQDs & 5 & $41.6 \pm 0.9$ & $454 \pm 37$ & $63 \pm 6$ & $58 \pm 5$ & $\mathrm{NA}^{\mathrm{b}}$ \\
\hline
\end{tabular}

${ }^{a} \mathrm{P} \leq 0.05$ compared with sham-operated mice. ${ }^{\mathrm{b}} \mathrm{NA}$ : not applicable. 

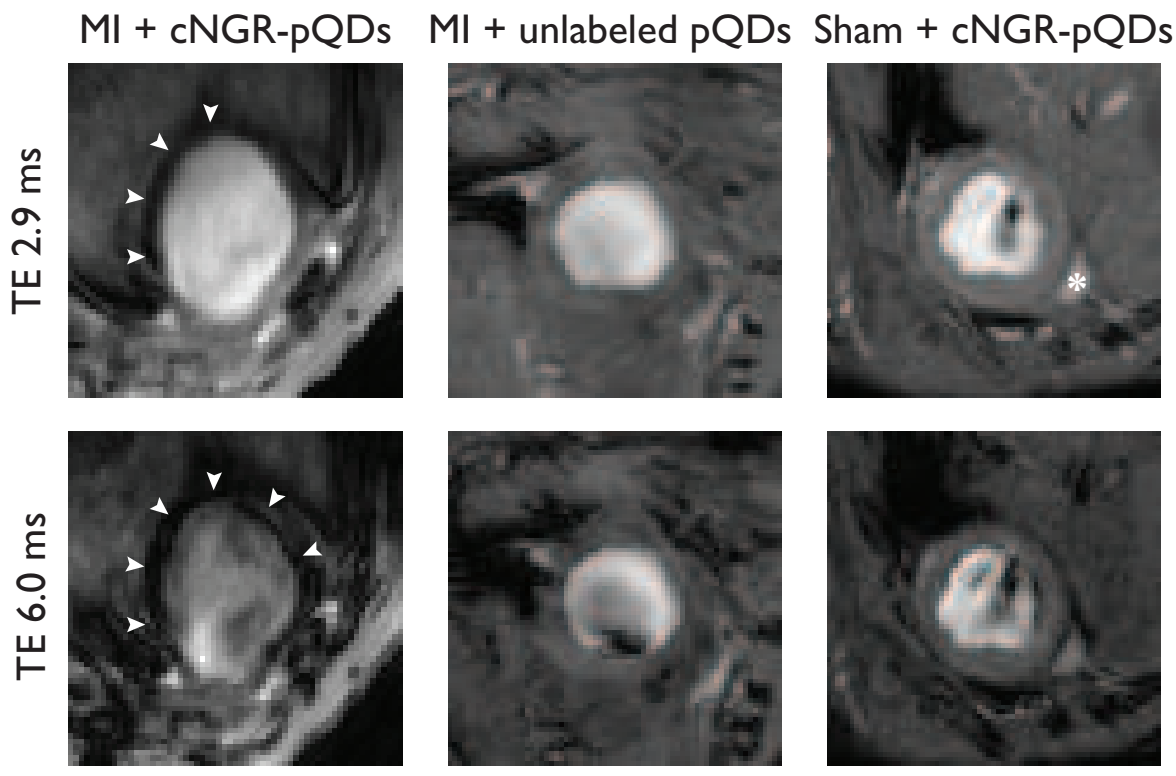

Figure 7.2: Short axis gradient echo images with TE $2.9 \mathrm{~ms}$ (top row) and TE $6.0 \mathrm{~ms}$ (bottom row) for an Ml-mouse injected with cNGR-pQDs (left), an MI-mouse injected with unlabeled pQDs (middle), and a sham-operated mouse injected with cNGR-pQDs (right). Short TE images allow better visualization of the anatomy, whereas the longer TE images show stronger $\mathrm{pQD}$-induced negative contrast. A large hypointense area is seen for Ml-mice injected with cNGR-pQDs (arrowheads), which is much smaller or absent in the other groups. In this specific case, the infarction extended also into the posterior wall. Note that for shamoperated mice, the LV is not dilated and the RV is therefore visible on short axis images (asterisk in upper right panel).

\section{Contrast agent uptake on molecular MRI}

Figure 7.2 shows short axis gradient echo images at 60 minutes post-contrast administration for a representative mouse of each experimental group. A large hypointense area was observed in the myocardium of Ml-mice injected with cNGR-pQDs, which was much smaller or absent in Ml-mice injected with unlabeled pQDs or in shamoperated mice injected with cNGR-pQDs. Any blood pool effect of circulating contrast agent was excluded based on the plasma half life, which was approximately 7 minutes for both cNGR-labeled and unlabeled pQDs, as estimated previously using dynamic MRI (Chapter 5) and fluorescence microscopy.

Images recorded with the shortest echo time of 2.9 ms allowed good visualization and delineation of the LV myocardium, whereas images recorded with an echo time of $6.0 \mathrm{~ms}$ were more suitable to determine the size of the hypointense area. In addition, gradient echo images with two echo times allowed estimation of the apparent transverse relaxation time $T_{2}^{*}$, which was approximately $10 \mathrm{~ms}$ in healthy myocardium. 


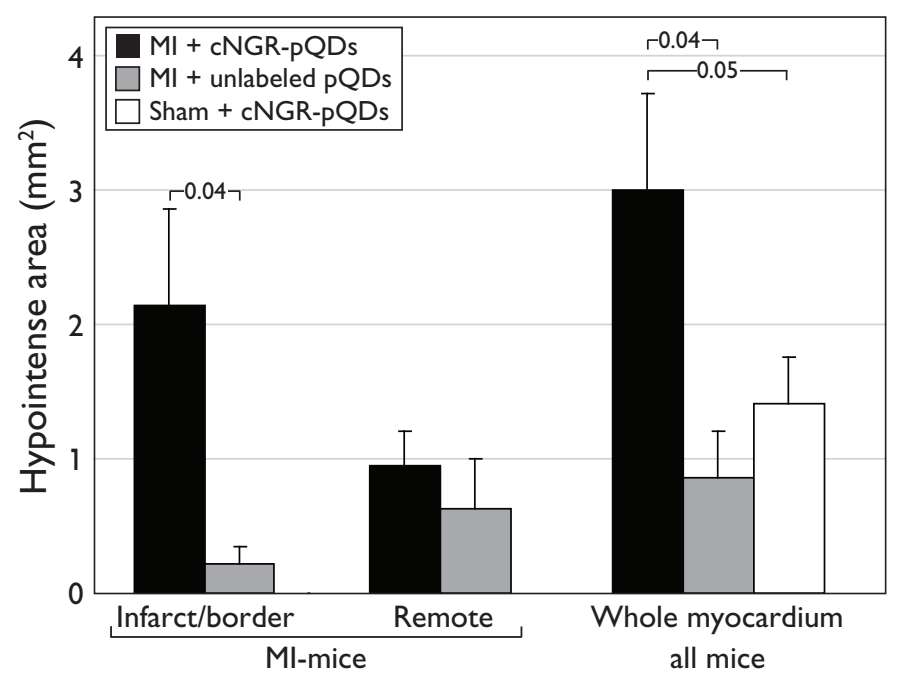

Figure 7.3: Average size of the hypointense area as measured on bright blood gradient echo images with TE $6.0 \mathrm{~ms}$ for the infarct/border zone, remote area, and whole myocardium. The infarct/border zone and remote area were defined by segmental analysis as explained in Figure 7.1. The whole myocardium was considered for comparison with sham-operated mice as no segmentation was performed in these mice. Data for Ml-mice injected with cNGR-pQDs $(n=6)$, MI-mice injected with unlabeled pQDs $(n=4)$, and sham-operated mice injected with cNGR-pQDs $(n=5)$ are indicated in black, grey, and white, respectively. Statistically significant $\mathrm{P}$-values are shown as well.

To link local cardiac function with the molecular MRI response, the myocardium was divided into 8 radial segments [223]. In each segment, contractile function was measured via the ERS and categorized as infarct/border zone or remote myocardium (Figure 7.1C). Next, the size of the hypointense area was quantified for each radial segment. For Ml-mice injected with cNGR-pQDs, the hypointense area was mostly located in the infarct/border zone and to a much lesser extent in the remote area (Figure 7.3). Furthermore, the hypointense area in the infarct/border zone was significantly larger for MI-mice injected with cNGR-pQDs compared with unlabeled pQDs, indicating a higher local contrast agent concentration in the former. No significant differences were found between the two groups in the remote myocardium. For comparison with sham-operated mice, the whole myocardium was considered as no segmentation was performed in these mice. A larger hypointense area was found for MI-mice injected with cNGR-pQDs than for sham-operated mice (Figure 7.3). Furthermore, cNGR-pQDs were retained in the myocardium of Ml-mice. At 2 hours post contrast administration, the hypointense area of MI-mice injected with cNGRpQDs was similar to 1 hour post contrast (whole myocardium $3.0 \pm 0.7$ and $2.4 \pm$ $0.5 \mathrm{~mm}^{2}$, respectively, $\mathrm{P}=0.5$ ). 

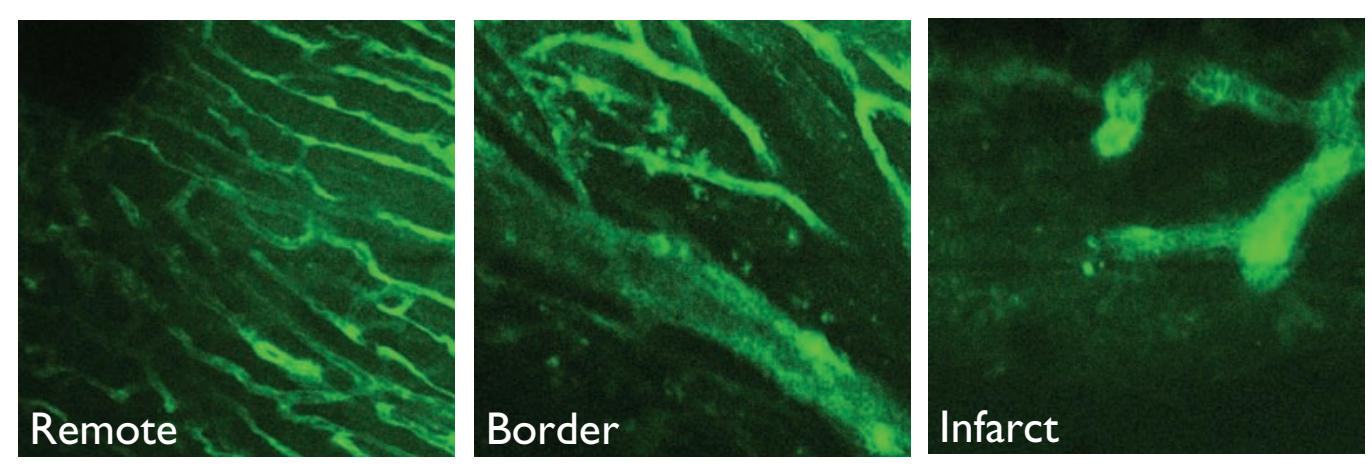

Figure 7.4: Representative TPLSM images showing vessel architecture in the remote myocardium, border zone, and infarct area. Vessels were stained in green using anti-CD31-FITC. Image size: $179 \times 179 \mu \mathrm{m}^{2}$.

\section{Microvascular structure and remodeling}

TPLSM images recorded in the infarct area, border zone, and remote myocardium of infarcted hearts showed remarkable differences in microvascular morphology using an all-vessel staining (Figure 7.4). In remote regions, numerous small, parallel oriented vessels were found, following the orientation of the cardiomyocytes [143]. In contrast, the number of vessels was 2- to 3-fold lower in the infarcted area, which appeared rather heterogeneous and also contained avascular regions. Furthermore, vessels in the infarcted area lacked a distinct orientation and had a 2-fold larger diameter than in the remote region. In the border zone, a mixed morphology was observed: both small and larger vessels were found and the organization was less structured compared with remote regions. Taken together, these findings clearly reveal microvascular remodeling in the infarcted and border zones in response to myocardial ischemia.

\section{Contrast agent localization}

Both cNGR-labeled and unlabeled pQDs were located in the infarct and border zones, while being only sparsely detected in the remote myocardium (Figure 7.5). Infarct and border zone could not be distinguished by QD contrast or localization, but was rather apparent from vascular morphology. Therefore, these two regions were grouped in the subsequent analysis.

In the infarct/border zone, cNGR-pQDs were mainly found to colocalize with vascular endothelial cells (Figure 7.5), which concurs with earlier results [143]. Although intra- and extravascular cNGR-pQDs were also observed, these were 3 times less frequent. In contrast, unlabeled pQDs were mainly observed in the extravascular space, with intravascular or colocalized unlabeled pQDs being 2-fold less frequent. TPLSM data therefore support that cNGR-pQDs bind specifically to activated endothelial cells of angiogenic vessels in the infarct/border zone of the myocardium, 
while unlabeled pQDs do not. In sham-operated mice, considerably less cNGR-pQDs were found compared with MI-mice. If present, cNGR-pQDs were found to be intravascular and not colocalized with endothelial cells.

\subsection{Discussion}

\section{Current findings}

Here, we demonstrated the applicability of molecular MRI to detect myocardial angiogenesis in vivo in a murine model of acute Ml. Compared with standard delayed enhancement imaging, which can only detect infarct size and location based on passive extravasation and abnormal wash-in and wash-out kinetics of a small contrast agent in the infarcted tissue [232], the presented method provides unique information on the angiogenic status of the myocardium. First, a strong negative contrast effect was observed in infarcted hearts using cNGR-labeled pQDs, which was mostly absent in sham-operated mice or in MI-mice injected with unlabeled pQDs. Second, quantification of local contractile function demonstrated that the hypointense area was mainly located in the infarct/border zone of the myocardium. Any presence of cNGR-pQDs in the remote myocardium might be related to the development of myocardial hypertrophy, since an increase in muscle tissue is likely accompanied by expansion of the vascular network, and hence, angiogenic activity. Third, administration of cNGR-pQDs resulted in a larger hypointense area in the infarct/border zone than unlabeled pQDs, indicating specific accumulation of cNGR-pQDs in this myocardial region. This corresponds with intravital microscopy results, where injection of a cNGR-labeled fluorophore resulted in a significantly higher signal in the infarct/border zone compared with unlabeled fluorophore [143]. Fourth, TPLSM investigation revealed a remarkably different vascular morphology in the infarct and border zone compared with remote myocardium, indicating vascular adaptation and remodeling in response to myocardial ischemia. This concurs with results of Grass et al., who described a transition into vascular remodeling with relatively large caliber vessels crossing the infarct area at 7 days post-MI [233]. Fifth, TPLSM showed that both cNGR-pQDs and unlabeled pQDs were present in the infarction and border zone. However, cNGR-pQDs were found to colocalize with vascular endothelial cells, whereas unlabeled $p Q D$ s were mostly found in the extravascular space. This further supports the specificity of cNGR-pQDs for angiogenic vessels in the heart. Taken together, these results demonstrate that cNGR-pQDs are a suitable contrast agent to selectively detect post-MI angiogenesis in the heart using in vivo molecular MRI and ex vivo TPLSM. 

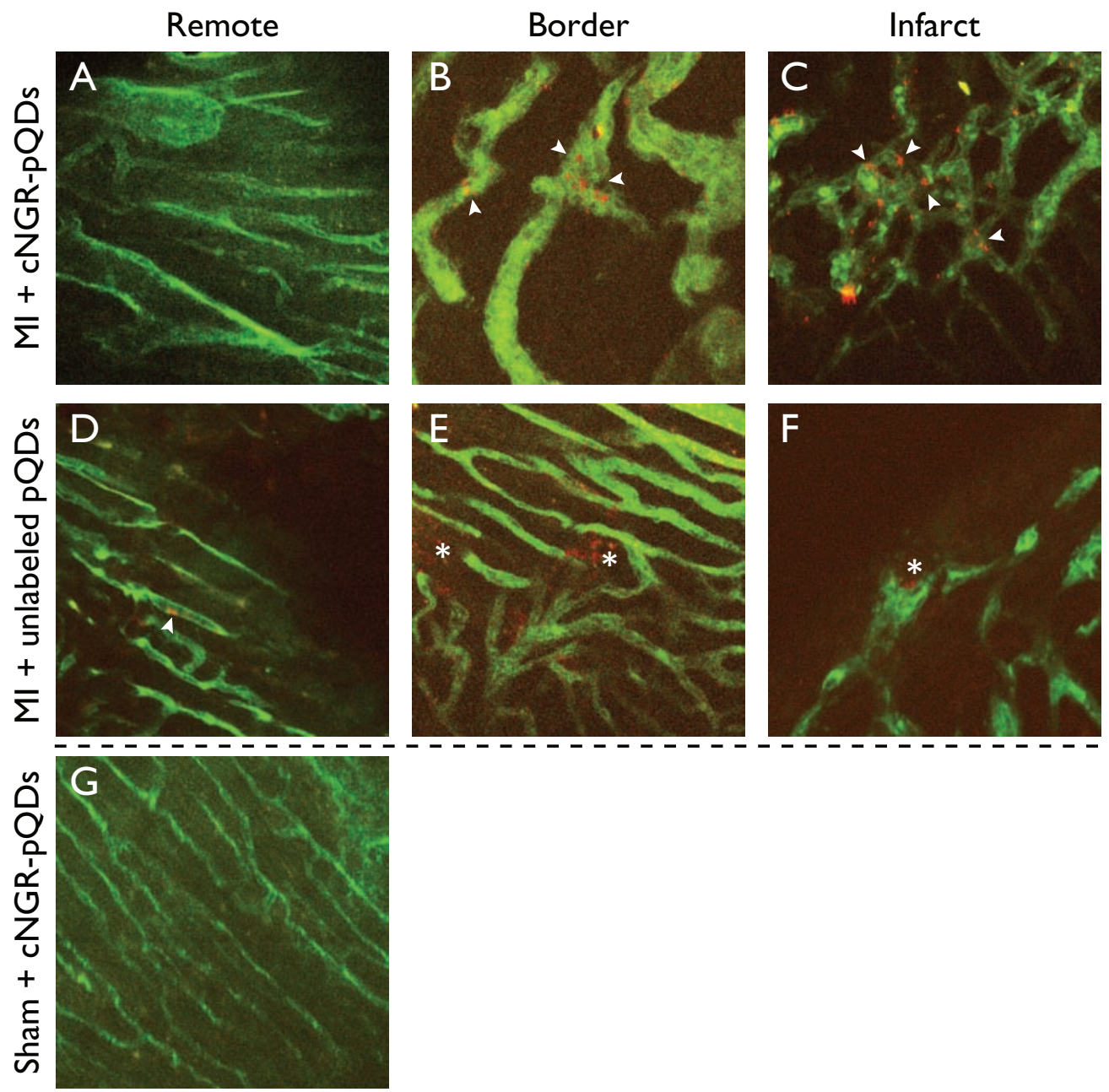

Figure 7.5: TPLSM images of an MI-mouse injected with cNGR-pQDs (A-C), an MI-mouse injected with unlabeled pQDs (D-F), and a sham-operated mouse injected with cNGR-pQDs $(G)$, for the remote myocardium $(A, D)$, border zone $(B, E)$, and infarct area $(C, F)$. The dashed line indicates that tissue classifications are not applicable to sham-operated mice. Vessels were stained in green using anti-CD31-FITC, whereas the signal from pQDs is shown in red. Arrowheads and asterisks denote colocalized and extravasated pQDs, respectively. For both MI groups, pQDs were mainly found in the border zone and infarct areas. Colocalization was most often observed for cNGR-pQDs, whereas unlabeled pQDs were found to extravasate more. In sham-operated mice (G), less cNGR-pQDs were observed compared with MI-mice. Image size: $179 \times 179 \mu \mathrm{m}^{2}$. 


\subsection{DISCUSSION}

\section{Contrast agent}

QDs were applied as contrast agent scaffold to allow non-invasive molecular MRI of the infarcted murine heart, validated by postmortem TPLSM. Although cNGRlabeled pQDs could selectively detect myocardial angiogenesis, the method cannot be translated directly into clinical trials. QDs have a cadmium-selenium core, accumulate mainly in the liver and spleen (Figure 3.4), and long-term toxicity is still unknown. Nevertheless, the current data demonstrate that cNGR selectively targets the contrast agent to angiogenic neovessels in the heart, which corresponds with previous studies [143]. Furthermore, validation of the MRI results with a subcellular resolution technique such as TPLSM is a necessary step in the development and evaluation of novel contrast agents. Once the targeting efficacy is fully established, cNGR could be coupled to more clinically applicable particles such as USPIOs (ultrasmall superparamagnetic iron oxide particles). USPIOs generate sufficient changes in image contrast, are cleared from the body, and were proven to be safe, even in patients with impaired renal function [234]. The applicability of cNGR-labeled USPIOs for cardiac imaging will be a topic of future research.

Although pQDs were originally designed as a positive contrast agent by labeling QDs with multiple Gd-DTPA moieties, as observed in previous tumor angiogenesis studies (Chapter 3), a strong negative contrast was observed in the myocardium. We suggest the following explanation. First, for proper $T_{1}$-weighting in gradient echo imaging and consequent positive contrast, $T_{2}^{*}$-relaxation effects can be minimized by setting the echo time much lower than tissue $T_{2}^{*}$. With echo times of 2.9 (shortest possible) and $6.0 \mathrm{~ms}$, and a tissue $T_{2}^{*}$ of $10 \mathrm{~s}$, this was clearly not the case. Second, a hypothetical relaxation mechanism leading to negative contrast is presented in Figure 7.6.

Tissue edema was shown to result in higher intrinsic $T_{1}$ and $T_{2}$ relaxation times in infarcted myocardium compared with remote areas $[235,236]$. Therefore, any small contrast agent induced reduction in $T_{1}$ or $T_{2}$ in the infarcted/border zones may have been cancelled by these intrinsic relaxation time differences. In contrast, the intrinsic $T_{2}^{*}$ was shown to be approximately $30 \%$ lower in infarcted myocardium compared with the remote area due to increased capillary recruitment [237]. Combined with the $T_{2}^{*}$-shortening effect of the contrast agent, this may have resulted in an enhanced contrast that exceeded the detection limit of MRI. The intrinsic differences in relaxation times between infarcted and healthy myocardium may therefore explain the absence of any detectable $T_{1}$-contrast and why $T_{2}^{*}$-contrast was more dominant to detect the presence of cNGR-pQDs in the heart.

\section{Molecular imaging}

Besides imaging angiogenic activity via endothelial cell targeting, other processes can be exploited for cardiac molecular imaging, including matrix metalloproteinase activity 
$[238,239]$, blood coagulation factor XIII related to infarct healing and ventricular remodeling [240], cardiomyocyte apoptosis [241-243], and fibrosis [229]. However, a major advantage of endothelial cell targets is their easy accessibility from the blood stream, resulting in relatively quick binding of the intravascular contrast agent to the target and high local concentrations. This is especially important in light of the short plasma circulation time of the currently applied contrast agent. From a clinical viewpoint, fast target binding and plasma clearance is also advantageous, as it would allow the complete examination to be finished within a clinically acceptable time frame. In contrast, imaging of an intracellular target would be performed at least 24 hours post contrast administration to allow sufficient contrast agent uptake, as is for instance seen in lymph node imaging [244].

An important issue regarding molecular imaging of the human heart is the spatial resolution of clinical MRI (approximately $1 \mathrm{~mm}$ ) versus nuclear imaging (5$10 \mathrm{~mm}$ [245]). This implies that clinical MRI, in contrast to nuclear methods, can spatially resolve pathologically different regions/features in the radial direction of the myocardial wall, detect infarct size and transmural extent, as well as the adjacent area at risk, and localize the expression of angiogenic biomarkers with respect to morphological details of the infarcted myocardium. As the area at risk is the part of the myocardium that is potentially salvageable upon revascularization, accurate detection of this area could have implications for the therapeutic strategy.

\section{Practical applications}

Several future (pre)clinical applications can be envisioned for molecular MRI of myocardial angiogenesis. First, it provides information on global and local cardiac function (e.g. EF and ERS), and the level of angiogenic activity within a single imaging session. It may therefore be applied for the early, in vivo evaluation of the response to angiogenic treatments in both preclinical and clinical studies. In addition, molecular MRI may provide a surrogate marker of therapeutic efficacy and can serve as a timely end-point in clinical trials. Second, future improvements in myocardial perfusion and function might be predicted based on the early angiogenic response. Third, measurement of baseline angiogenic activity may allow risk stratification, development of individualized therapy, and patient selection for clinical trials. One might argue that patients with a limited level of angiogenesis have the largest need for pro-angiogenic therapy and may therefore benefit the most. However, it has also been suggested that these patients apparently have a failed natural angiogenic response and will therefore be more resistant to exogenous angiogenic stimuli [221]. Fourth, conjugation of an imaging label to pro-angiogenic drugs may allow monitoring of drug delivery. Fifth, the presented method to detect angiogenesis in the mouse heart opens the way for studies in transgenic/knock-out animals, which may provide novel insights into the mechanisms of vascular adaptation and remodeling in the heart, and the roles of individual genes in these processes. 
Quiescent vessel

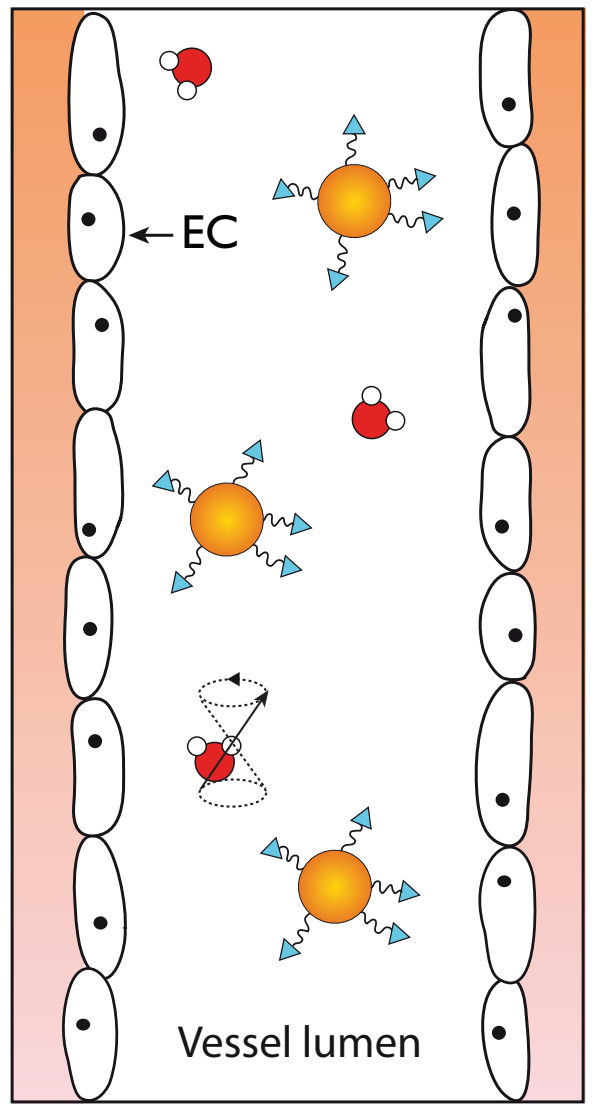

Angiogenic vessel

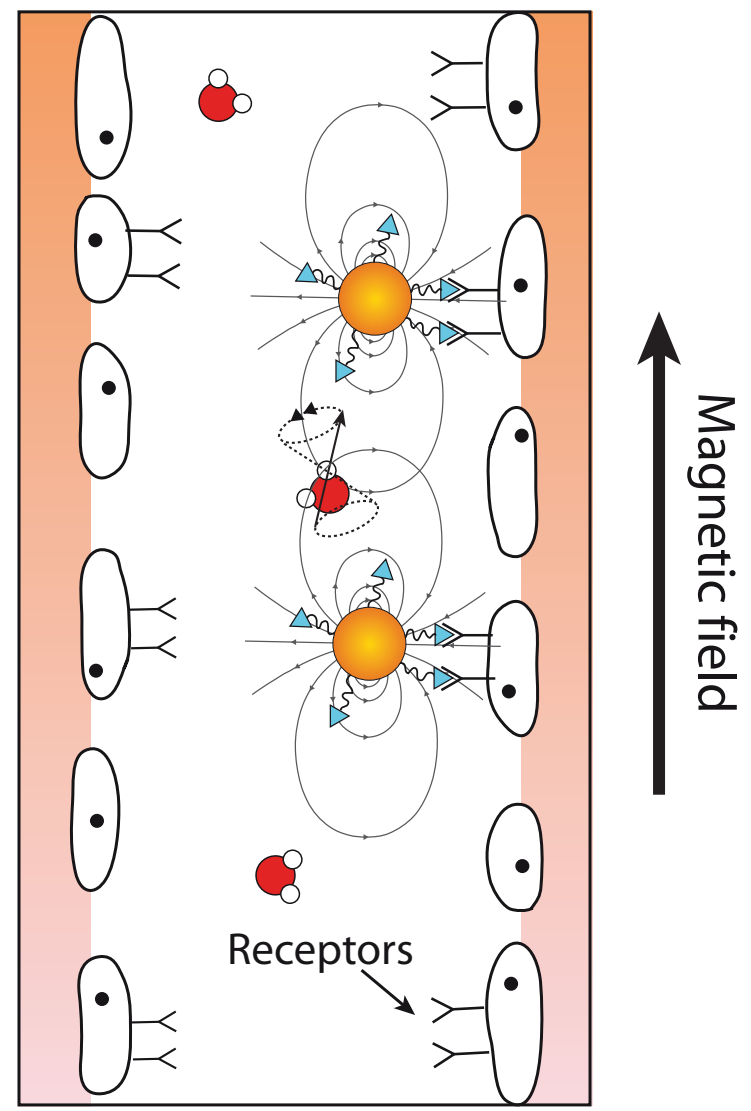

Figure 7.6: Hypothetical negative contrast mechanism for cNGR-pQDs. Vascular endothelial cells in quiescent vessels (left panel) form a nicely aligned network and show no upregulation of specific receptors. The targeted contrast agent flows freely through the vasculature and the magnetic properties of $\mathrm{pQDs}$ have a relatively weak effect on the water proton resonance frequency. In angiogenic vessels (right panel), endothelial cells display upregulated receptors to which the targeted agent can bind with high affinity. This results in high local contrast agent concentrations, which in turn generates static field perturbations due to the magnetic properties of $\mathrm{pQDs}$ [159] in combination with the dense gadolinium concentration on the QD surface. These field perturbations, which are aligned with the main magnetic field, likely result in an altered resonance frequency of nearby water protons and a consequently reduced signal intensity due to locally strong intravoxel dephasing effects of the water proton spins. Whether this negative contrast effect can be applied to generate positive contrast images using techniques like susceptibility gradient mapping [246] or white marker imaging [247] needs to be explored. Furthermore, these novel techniques need to be tailored to cardiac imaging. For unlabeled pQDs, there is no binding to upregulated receptors and contrast agent may extravasate through the permeable endothelial cell layer. Contrast agent distribution is relatively diffuse in the extravascular space and high local contrast agent concentrations are not reached. Consequently, negative contrast is not observed on the corresponding MR images. 


\section{CHAPTER 7. MOLECULAR MRI OF CARDIAC ANGIOGENESIS}

In conclusion, cNGR-labeled pQDs allowed specific detection of post-MI myocardial angiogenesis, as shown by the strong contrast observed in the infarcted mouse heart on molecular MR images, and by the colocalization of cNGR-pQDs with vascular endothelial cells as detected by TPLSM. In addition, TPLSM provided unique, detailed information on microvascular structure and remodeling in different regions of the heart. 
Part III

Renal microvasculature 



\section{8}

\section{MRI of renal oxygenation and function after normothermic ischemia-reperfusion injury}

Kidneys transplanted after cardiac death suffer from a high incidence of delayed graft function and primary non-function, likely caused by normothermic ischemia after cardiac arrest of the donor and subsequent reperfusion. In order to improve the clinical outcome of these kidneys, novel therapies are currently being developed that limit renal ischemia-reperfusion (I/R) injury. However, to fully address their therapeutic potential, non-invasive imaging methods are required which allow in vivo visualization of different renal compartments and longitudinal evaluation of kidney function after transplantation. Here, magnetic resonance imaging (MRI) was applied to study kidney oxygenation and function, in a murine model of renal I/R injury. Longitudinal oxygenation measurements showed a significantly reduced oxygenation of the outer medulla of the injured kidney compared with the contralateral control, which persisted up to 24 hours post reperfusion. In contrast, the oxygenation level of the inner medulla returned to baseline within 1 hour, whereas the cortex showed a slight but significant increase in oxygenation after $\mathrm{I} / \mathrm{R}$ injury. Ongoing ischemic injury was further supported by dynamic contrast-enhanced MRI, which revealed a significantly reduced renal function, comprising perfusion and filtration, in the I/R injured kidney 24 hours after reperfusion. Extensive I/R injury was confirmed by histology. In conclusion, MRI is suitable for the non-invasive, longitudinal evaluation of renal oxygenation and function. Even at 24 hours after reperfusion, oxygenation and function were significantly reduced in the I/R injured kidney, indicating ongoing ischemic damage. These time and location dependent MRI features may be used to monitor therapeutic intervention and to predict the outcome of transplanted kidneys from donors with cardiac arrest.

Submitted 


\subsection{Introduction}

Kidney transplantation is the preferred treatment for end-stage renal disease. Although the number of available kidneys is insufficient, the donor pool can be considerably expanded via donation after cardiac death [248]. However, these donors typically undergo a variable period of normothermic ischemia from the time of circulatory arrest to the time of intravascular flushing, causing ischemic injury to the kidneys. This results in acute renal failure (ARF), and a high incidence of delayed graft function and primary non-function after transplantation [248]. After reperfusion, there is a severely reduced blood flow, stasis, and accumulation of red and white blood cells [249], likely resulting in ongoing ischemic damage to the transplanted kidney. Of the different functional regions in the kidney, the outer medulla is especially vulnerable to ischemic damage because of its inherent hypoxic environment [250].

To improve the clinical outcome of kidneys transplanted after cardiac death, novel therapies, such as growth factor and cell therapies, are being developed that limit ischemia-reperfusion (I/R) injury [251]. However, to fully assess their therapeutic potential non-invasively in an early stage, new imaging methods are required that allow evaluation of the function of different renal compartments in a longitudinal manner in both preclinical and clinical setups. In addition, longitudinal monitoring of renal performance may provide more insight into long term pathophysiological development, which in turn may aid in the improvement of therapies directed against I/R injury and ARF.

Magnetic resonance imaging (MRI) is the most versatile non-invasive imaging method available today. Advantages are a superior spatial resolution and soft tissue contrast, which allows differentiation between specific anatomical regions of the kidney (i.e. cortex, inner and outer medulla). In addition, MRI uses no ionizing radiation or nephrotoxic contrast agents and can therefore be safely repeated in the same patient. Here, two complementary MRI techniques, i.e. blood oxygen level dependent (BOLD) MRI and dynamic contrast-enhanced (DCE) MRI, were applied to study renal oxygenation and perfusion, respectively, in a murine model of $I / R$ injury.

BOLD-MRI was shown to be useful in evaluating intrarenal oxygenation in humans [252-256] and in rodents $[257,258]$. It is based on susceptibility differences between oxyhemoglobin and deoxyhemoglobin. While the former is diamagnetic, the latter contains an even number of unpaired electrons, which makes it paramagnetic [259]. When the tissue oxygenation state is altered, the ratio between oxyhemoglobin and deoxyhemoglobin concentrations will change accordingly, which results in a local change in magnetic susceptibility. These differences can be detected via changes in the apparent transverse relaxation rate $R_{2}^{*}\left(=1 / T_{2}^{*}\right)$, where an increase in $R_{2}^{*}$ indicates a decreased oxygenation and vice versa. In the kidney, BOLD measurements were found to be highly reproducible and robust [260]. Furthermore, cortical and medullar $R_{2}^{*}$ as detected by BOLD-MRI were found to be linearly related to renal $\mathrm{pO}_{2}$ as measured using invasive microelectrodes [261]. In addition, BOLD- 
MRI allowed differentiation between acute rejection and tubular necrosis after renal transplantation [262].

BOLD-MRI cannot distinguish between changes in $R_{2}^{*}$ caused by altered tissue oxygenation, perfusion, or oxygen consumption [255]. Therefore, DCE-MRI measurements were included in the present study to estimate local renal perfusion. DCE-MRI measurements aim to detect abnormal contrast agent wash-in and washout kinetics in diseased tissue compared with healthy tissue. Subsequent analysis of the dynamic signal enhancement curves using pharmacokinetic multi-compartment modeling provides quantitative information on tissue perfusion, which is independent on inflow effects, bolus length, or cardiac function. Although DCE-MRI was primarily developed for oncological purposes, it has also been successfully applied to study normal renal function in humans [263-265]. However, only few papers described the application of DCE-MRI in diseased kidneys [266], or in animal models of kidney disease $[267,268]$.

Here, we applied non-invasive MR imaging to demonstrate the feasibility of longitudinal monitoring of renal oxygenation in various regions of the kidney using a murine model of I/R injury. In addition, we showed for the first time that ongoing ischemic injury, comprising impaired renal oxygenation and function, can be detected non-invasively at a relatively late time point during renal I/R injury, i.e. 24 hours after reperfusion.

\subsection{Methods}

\section{Animal model}

All animal experiments were approved by the institutional ethics committee on the care for experimental animals. Nine male Swiss mice were anesthetized using 5\% isoflurane (Abbott Laboratories Ltd, Queensborough, UK) in medical air. Animals were transferred to a dedicated animal bed with built-in mask for anesthesia gas supply during the MRI measurements. Body temperature and respiration rate were continuously monitored via an MR compatible small animal monitoring system (SA Instruments, Stony Brook, NY). Normothermic conditions were maintained using a warm water heating pad. After baseline MRI measurements, mice were transferred to an operating area outside the magnet room. Pre-operative analgesia was induced by administrating buprenorphine (Temgesic ${ }^{\circledR}$, Schering-Plough, Utrecht, The Netherlands) $0.1 \mathrm{mg} / \mathrm{kg}$ subcutaneously. Next, the left renal pedicle was exposed and clamped using a non-traumatic MR-compatible surgical clamp (Vascu-Statt ${ }^{\circledR}$ midi angled 1001-501, Scanlan International, St Paul, MN), as a model of renal I/R injury. Mice were transferred back into the MRI scanner, where images were obtained during ischemia. After 45 minutes of ischemia, the clamp was removed and images were obtained as soon as possible (mean 10 minutes after reperfusion) and at 1 hour after 
reperfusion. After the 1 hour scan, the wound was closed in two layers and buprenorphine $0.25 \%$ was applied topically for postoperative pain management. Next, images were obtained at 5 and 24 hours after reperfusion. Animals were allowed to recover after imaging at 1 and 5 hours post reperfusion. For the final measurement session, an infusion line was placed in the tail vein to allow contrast agent injection during the MRI exam. After MRI, mice were sacrificed and kidneys were excised and fixed in formalin for histological analysis.

\section{MRI protocol}

All MR experiments were performed on a 7 Tesla Bruker Biospec 70/30 USR MRI scanner (Bruker Biospin GmbH, Ettlingen, Germany), interfaced to an AVANCE II console. The BGA12-S mini-imaging gradient system was used (maximum gradient strength $720 \mathrm{mTm}^{-1}$, slew rate $6000 \mathrm{Tm}^{-1} \mathrm{~s}^{-1}$ ) and images were acquired using a $3.5 \mathrm{~cm}$ inner diameter quadrature volume resonator.

Kidneys were localized using axial $T_{1^{-}}$and $T_{2}$-weighted spin echo images covering both kidneys completely. The repetition and echo times (TR/TE) for $T_{1}$ and $T_{2}$ weighted images were $1500 / 7.6$ and $3000 / 40$ ms, respectively. The field-of-view was $4 \times 4 \mathrm{~cm}^{2}$, which together with a $256 \times 256$ acquisition matrix resulted in an inplane resolution of $0.16 \times 0.16 \mathrm{~mm}^{2}$. On average, $191 \mathrm{~mm}$ thick slices were recorded (range 16-20, depending on kidney orientation). Four signal averages were acquired to reduce motion artifacts.

\section{BOLD-MRI}

The apparent transverse relaxation rate $R_{2}^{*}$ was measured using a multi-slice multiecho gradient echo sequence (MGE) with TR $750 \mathrm{~ms}$, TE ranging from 4 to $34 \mathrm{ms,}$ with an echo interval of $6 \mathrm{~ms}$, and a $30^{\circ}$ flip angle. Geometrical image parameters were similar to the anatomical images and 4 signal averages were obtained.

\section{DCE-MRI}

Dynamic imaging was performed using a gradient echo sequence with a TR and TE of 65 and $1.9 \mathrm{~ms}$, respectively, and a $35^{\circ}$ flip angle. To reduce scan time, the acquisition matrix was reduced to $128 \times 128$, and only 10 axial slices were recorded with 2 signal averages. In total, 100 dynamic phases were acquired with a $16 \mathrm{~s}$ temporal resolution. Injection of $0.1 \mathrm{mmol} / \mathrm{kg}$ Gadovist $^{\circledR}$ (Bayer Schering Pharma AG, Berlin, Germany), diluted to a total volume of $100 \mu \mathrm{L}$, was started at phase 11 and followed by a $50 \mu \mathrm{L}$ saline flush. The total injection duration was approximately 20 seconds. 


\section{MRI analysis}

All data analysis was performed in Matlab (The MathWorks, Natick, MA), unless stated otherwise. Regions of interest (ROIs) were drawn manually in MRIcro [152] using both $T_{1^{-}}$and $T_{2}$-weighted anatomical images for correct delineation of the whole kidney and the different kidney regions.

\section{BOLD-MRI}

ROls were drawn in the cortex, outer medulla, and inner medulla in three slices through the center of each kidney (Figure 1 ). $R_{2}^{*}$ values were determined on a voxelby-voxel basis via nonlinear fitting of the gradient echo signal intensity function [153]:

$$
S=S_{0} \cdot e^{-\mathrm{TE} \cdot R_{2}^{*}}
$$

using the Levenberg-Marquardt optimization algorithm. Here, $S_{0}$ is a scaling factor depending on proton density, TR, flip angle, pulse profile, and preamplifier gain.

\section{DCE-MRI}

First, dynamic data were spatially coregistered using the rigid body transformation algorithm in SPM2 (Wellcome Trust Centre for Neuroimaging, London, UK) to correct for possible animal motion during data acquisition. Second, the relative signal intensity changes $\Delta S(t)$ were calculated per voxel as $\Delta S(t)=S(t) / S_{\text {pre }}-1$, with $S_{\text {pre }}$ the average signal intensity of the 10 pre-contrast phases. Third, using phantoms with relatively low gadolinium concentrations ranging from $0-1.0 \mathrm{mM}$, DCE-MRI signal intensities were found to be linearly related to gadolinium concentration. All phantoms contained $0.1 \mathrm{mM} \mathrm{MnCl} 2$ to mimic a background $T_{1}$ of muscle tissue $(\approx 1000 \mathrm{~ms})$. The linear coefficients as derived from the phantom experiment were used to convert in vivo $\Delta S(t)$ curves to $C(t)$ curves, with $C$ the gadolinium concentration in $\mathrm{mM}$. Fourth, dynamic tissue enhancement curves $C_{\text {tissue }}(t)$ were analyzed using the two-compartment filtration model of Sourbron et al. [264]:

$$
C_{\text {tissue }}(t)=V_{P} \cdot C_{P}+F_{T} \cdot e^{-t / T_{T}} \otimes C_{P}(t)
$$

Here, $V_{P}$ is the relative plasma volume, $F_{T}$ is the tubular flow, which was shown to be related to the glomerular filtration rate, and $T_{T}$ is the tubular transit time. $C_{P}(t)$ is the plasma gadolinium concentration in the kidney, which can be obtained from the arterial gadolinium concentration $C_{A}(t)$ according to:

$$
C_{P}(t)=T_{P}^{-1} \cdot e^{-t / T_{P}} \otimes C_{A}(t)
$$


with $T_{P}$ being the plasma transit time. The plasma flow $F_{P}$, which is related to renal perfusion, can be calculated as $V_{P} / T_{P}$. As no reliable arterial input function could be obtained from the abdominal aorta, the input function was obtained from the erector spinae muscle using a reference region model with vascular term [269]. The following values were assumed for the pharmacokinetic parameters of the reference muscle: $K^{\text {trans }}=0.15 \mathrm{~min}^{-1}, v_{\mathrm{p}}=0.025$, and $v_{\mathrm{e}}=0.1$. Voxels with an overall fit error of more than $50 \%$ were excluded from further analysis [158]. Note that the two-compartment model applies to the kidney as a whole or to the cortex, but not to the medulla $[264,265]$. Consequently, DCE-MRI analyses concerned only the whole kidney and no distinction was made between the 3 functional regions as for BOLD-MRI.

\section{Histology}

Kidneys were sectioned into $3 \mu \mathrm{m}$ slides and stained for histology with periodic acid Schiff (PAS). Based on the method of Leemans et al. [270], the extent of tubular damage in the corticomedullary junction was scored by an experienced nephropathologist, who was blinded to which experimental group the samples belonged, using a scale from 0 to 5 , representing no and extensive damage, respectively. The following criteria were assessed in 10 randomly chosen, non-overlapping fields: tubular dilatation, cast deposition, brush border loss, and necrosis.

\section{Statistical analysis}

All values are presented as mean \pm standard error, unless stated otherwise. Statistical analysis was performed using paired Student's t-tests in SPSS 17.0 (SPSS, Chicago, III). For DCE-MRI data, the parameters $F_{P}$ and $F_{T}$ were first combined according to O'Brien and Läuter $[156,157]$ to obtain an overall measure that is more sensitive to changes in renal function than the individual parameters. To this extent, values were standardized according to $z_{P, T}=\left(F_{P, T}-\operatorname{mean}\left(F_{P, T}\right)\right) / \operatorname{sd}\left(F_{P, T}\right)$. Next, $z_{P}$ and $z_{T}$ were averaged per kidney and the resulting summary measure was tested using a paired t-test. $\mathrm{P}<0.05$ was considered statistically significant.

\subsection{Results}

As a model of renal I/R injury, the left renal pedicle of male Swiss mice was clamped for 45 minutes. The contralateral kidney served as an internal control. Kidney size was measured using anatomical $T_{2}$-weighted images covering both kidneys completely. At 24 hours after reperfusion, no significant differences were found in either total kidney volume or cortical volume between clamped and control kidneys ( Table 8.1). 
Table 8.1: Renal and cortical volumes of clamped and control kidneys, as measured on $T_{2^{-}}$ weighted anatomical MR images. No significant differences were found between clamped and control kidneys $(P>0.05)$.

\begin{tabular}{lcc}
\hline & Clamped & Control \\
Renal volume $\left(\mathrm{mm}^{3}\right)$ & $541 \pm 21$ & $537 \pm 20$ \\
Cortical volume $\left(\mathrm{mm}^{3}\right)$ & $305 \pm 17$ & $285 \pm 16$ \\
\hline
\end{tabular}

\section{Renal oxygenation}

BOLD measurements of renal oxygenation and subsequent quantification of the tissue $R_{2}^{*}$ were performed at baseline, during ischemia, and at 10 minutes and 1,5 , and 24 hours after reperfusion. Figure 8.1 shows representative images of clamped and control kidneys from the same mouse, with color-coded overlay of the renal $R_{2}^{*}$ at different time-points during I/R injury. During ischemia, a clearly increased $R_{2}^{*}$ was observed in the clamped kidney, implying a strongly decreased oxygenation, which persisted after reperfusion.

To analyze oxygenation in the different functional regions of the kidney, regions of interest were defined in the cortex, inner medulla, and outer medulla, based on tissue contrast on $T_{1}$ - and $T_{2}$-weighted anatomical images (Figure 8.1). Care was taken to exclude large veins. Figure 8.2 shows the average course of renal oxygenation in these three functional regions during I/R injury. Compared with the control kidney, a significantly decreased oxygenation was found during ischemia for all regions of the clamped kidney, which persisted in the outer medulla at all investigated time-points after reperfusion. In contrast, in the cortex, a significantly increased oxygenation was found compared with the control kidney at 1, 5, and 24 hours post reperfusion, whereas in the inner medulla oxygenation returned to baseline level within 1 hour after reperfusion.

Linear regression analysis of the $R_{2}^{*}$ changes over time in the control kidney revealed no significant slopes (cortex and outer medulla) or a slope that was irrelevantly small $\left(\mathrm{d} R_{2}^{*} / \mathrm{dt}=-0.009 \mathrm{~s}^{-1} \mathrm{~min}^{-1}\right.$ for inner medulla, not shown) compared with the ischemic effect in the injured kidney.

\section{Renal function}

Renal function as assessed by DCE-MRI was performed at 24 hours post reperfusion only, since the gadolinium-based contrast agent also effects $R_{2}^{*}$ and may therefore have interfered with the longitudinal BOLD experiments. Figure 8.3 shows a representative example of dynamic gadolinium enhancement curves obtained in clamped and control kidneys. The clamped kidney showed an increased enhancement compared with the control kidney, and both kidneys showed a higher perfusion than muscle tissue. 


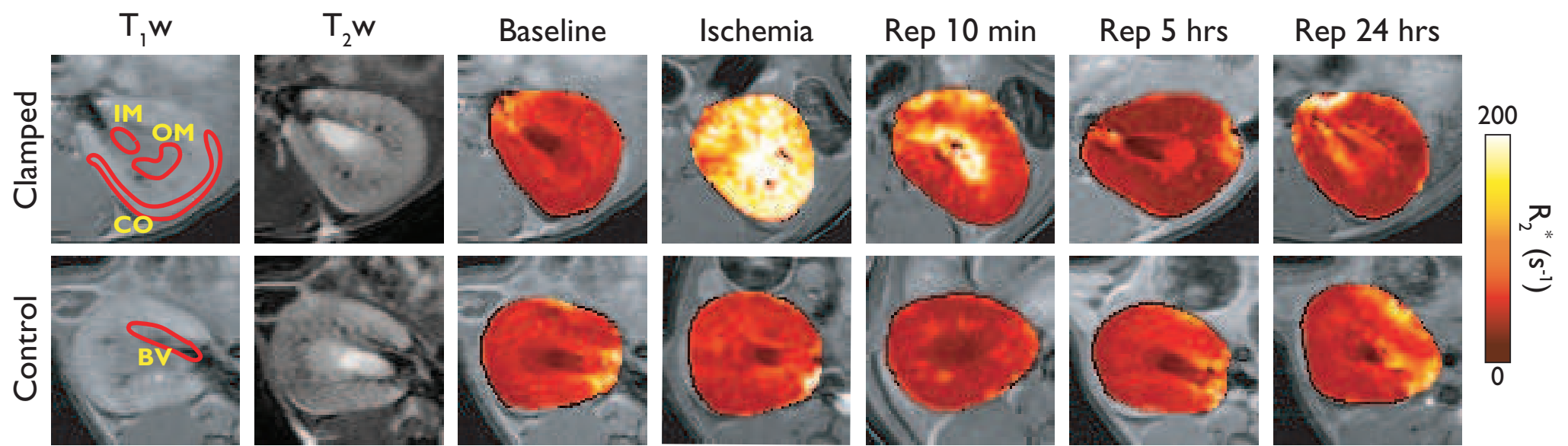

Figure 8.1: $T_{1}$ - and $T_{2}$-weighted anatomical images (left), with the different regions of interest indicated on the images. CO: cortex, IM: inner medulla, OM: outer medulla. Blood vessels (BV) were excluded from analysis. The remaining panels show color-coded overlays of renal $R_{2}^{*}$-maps for the clamped (top row) and the control kidney (bottom row) of the same mouse, recorded at different time-points during ischemia-reperfusion injury. Note that the shape of the kidneys and their surrounding anatomy differs between the images since the mouse was repositioned in the MRI scanner between each of the longitudinal images. 

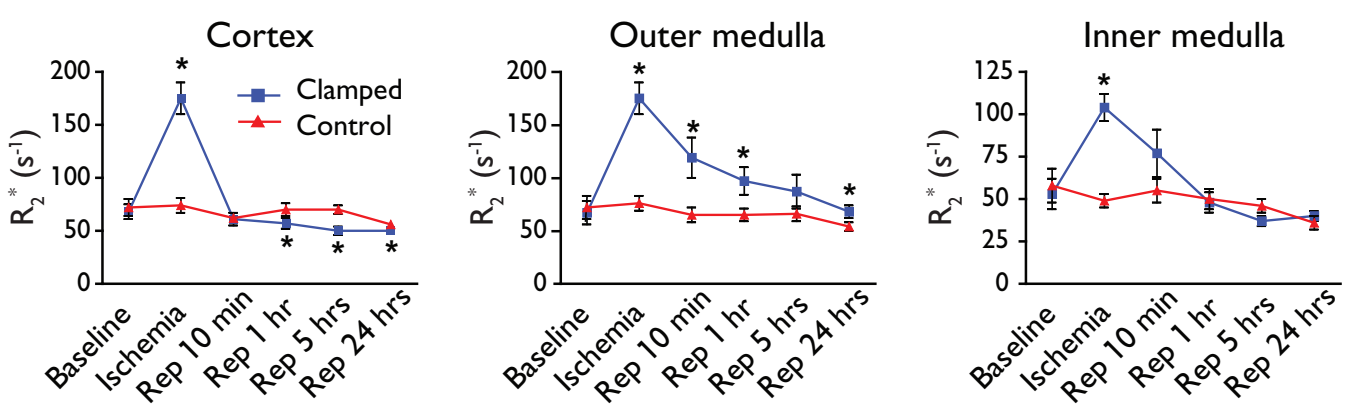

Figure 8.2: Average $R_{2}^{*}$ values at different time-points during ischemia-reperfusion injury in the cortex, outer medulla, and inner medulla of clamped (blue) and control (red) kidneys. $* \mathrm{P}<0.05$ compared with control kidney.

Next, dynamic enhancement curves were fitted using the two-compartment filtration model of Sourbron et al. [264], combined with a reference region input function [269]. From this model, the following functional parameters were derived: the renal plasma flow $F_{P}$, which is related to kidney perfusion; the tubular flow $F_{T}$, which is related to the glomerular filtration rate; the plasma transit time $T_{P}$; the tubular transit time $T_{T}$; and the fractional plasma volume $V_{P}$. Anatomical images of mouse kidneys with color-coded overlays of the perfusion and filtration parameters $F_{P}$ and $F_{T}$, respectively, are shown in Figure 8.4. Compared with the control kidney, a marked reduction in both perfusion and filtration was observed in the clamped kidney. Averaged values of all DCE-MRI derived functional parameters are summarized in Table 8.2. To obtain a summary measure that is more sensitive to changes in renal function than the individual parameters, $F_{P}$ and $F_{T}$ were combined according to the method of O'Brien and Läuter $[156,157]$. This summary measure indicated a significantly lower renal function in the clamped kidney compared with the contralateral control $(P=0.004$, Table 8.2).

Table 8.2: Whole kidney averaged pharmacokinetic parameters for clamped and control kidneys. An O'Brien-Läuter test of the combined functional parameters $F_{P}$ and $F_{T}$ resulted in a highly significant difference between clamped and control kidneys $(P=0.004) . F_{P}$ : plasma flow, related to renal perfusion; $F_{T}$ : tubular flow, related to renal filtration; $T_{P}$ : contrast agent plasma transit time; $T_{T}$ : contrast agent tubular transit time; $V_{P}$ : relative plasma volume.

\begin{tabular}{lcc}
\hline & Clamped & Control \\
$F_{P}\left(\mathrm{~mL} / \mathrm{min} / 100 \mathrm{~cm}^{3}\right)$ & $451 \pm 24$ & $481 \pm 26$ \\
$F_{T}\left(\mathrm{~mL} / \mathrm{min} / 100 \mathrm{~cm}^{3}\right)$ & $0.91 \pm 0.15$ & $1.04 \pm 0.16$ \\
$T_{P}(\mathrm{~min})$ & $0.039 \pm 0.001$ & $0.037 \pm 0.001$ \\
$T_{T}(\mathrm{~min})$ & $210 \pm 91$ & $301 \pm 60$ \\
$V_{P}\left(\mathrm{~mL} / 100 \mathrm{~cm}^{3}\right)$ & $17.69 \pm 1.1$ & $17.7 \pm 1.0$ \\
\hline
\end{tabular}




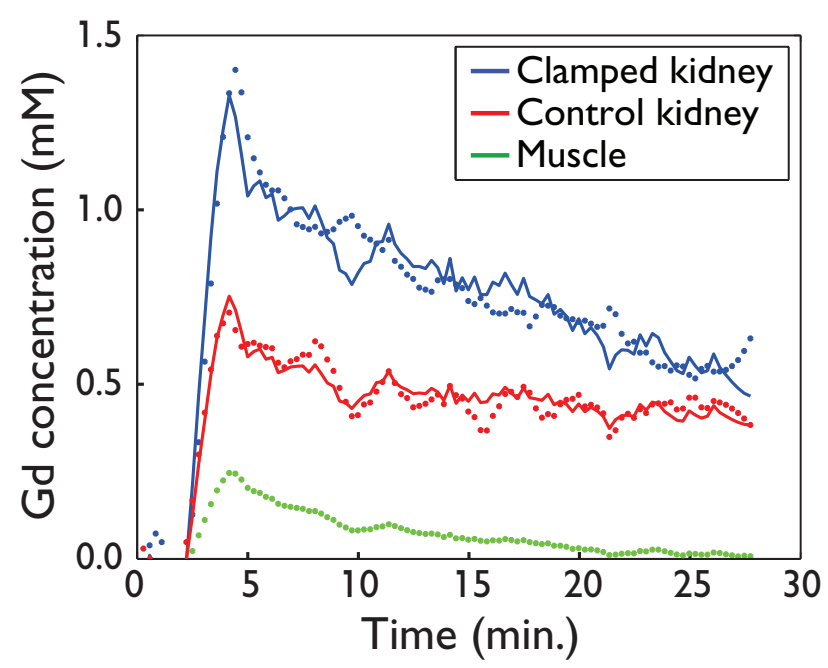

Figure 8.3: Dynamic gadolinium enhancement curves for the clamped kidney (blue), control kidney (red), and erector spinae muscle (green) of a representative mouse. The clamped kidney displays a stronger enhancement compared with the control kidney. Dotted lines represent the experimental data, whereas solid lines indicate the data fitted using the twocompartment filtration model.
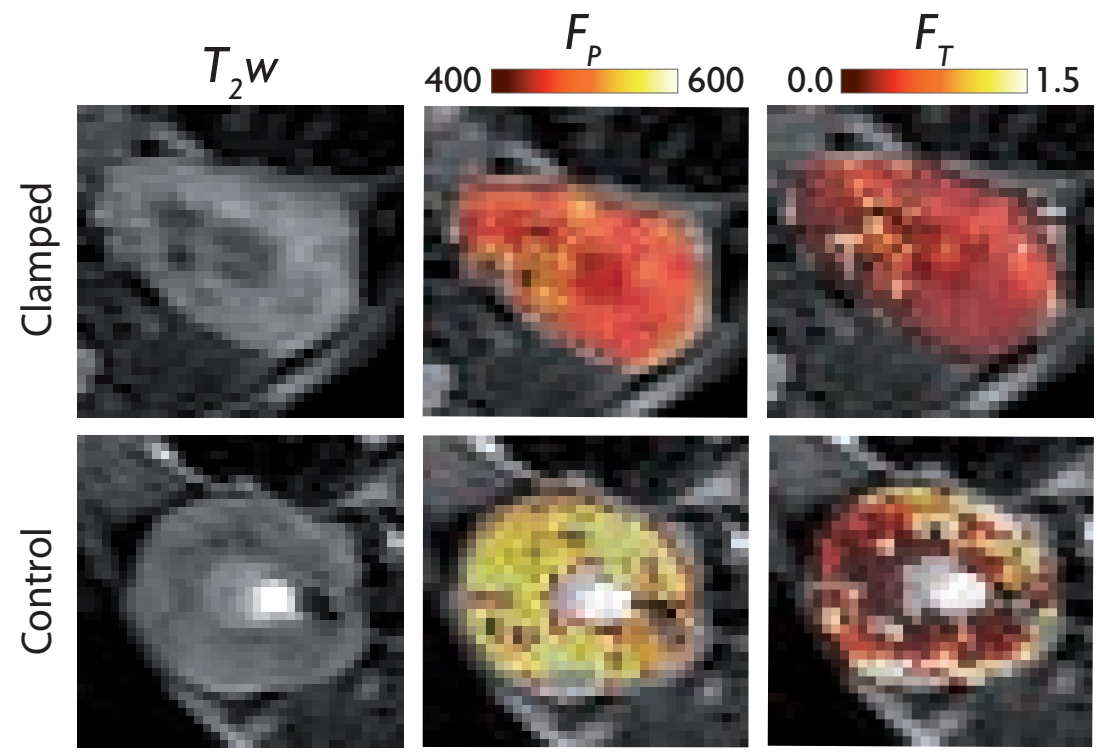

Figure 8.4: $T_{2}$-weighted anatomical image (left) with color-coded overlay of the perfusion parameter $F_{P}$ (middle, unit: $\mathrm{mL} / \mathrm{min} / 100 \mathrm{~cm}^{3}$ ) and the filtration parameter $F_{T}$ (right, unit: $\mathrm{mL} / \mathrm{min} / 100 \mathrm{~cm}^{3}$ ) for a clamped (upper panels) and control (lower panels) kidney of the same mouse. 


\section{Histology}

Histological PAS staining confirmed extensive I/R injury in the clamped kidneys, whereas negligible damage was found in contralateral control kidneys (Figure 8.5). The abundance of tubular dilatation, tubular casts, tubular necrosis, and loss of brush borders throughout a major part of the medulla resulted in high injury scores in the clamped kidneys, which is a typical result 24 hours after clamping.
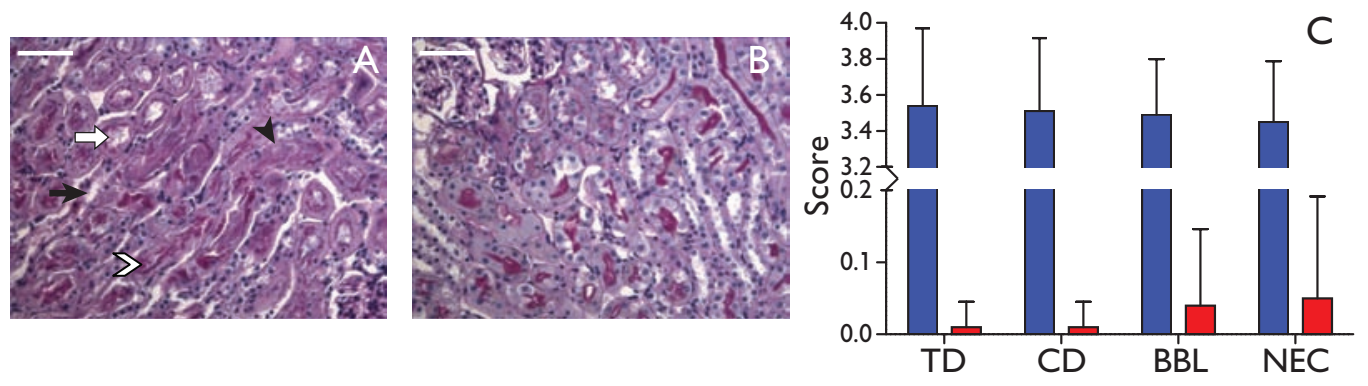

Figure 8.5: PAS-stained sections (magnification $100 \times$ ) of a clamped (A) and a control kidney (B) showing extensive injury of the clamped kidney. Open arrow: tubular dilatation (TD). Open chevron: cast deposition (CD). Black arrow: brush border loss (BBL). Black arrowhead: necrosis (NEC). Bar: $50 \mu \mathrm{m}$. C). Average histological scores of renal damage according to Leemans et al. [270]. Clamped and control kidneys are indicated in blue and red, respectively. Note the discontinuous $y$-axis, which was required to allow visualization of the extremely low values found in the control kidneys, compared with the high scores of the clamped kidneys. Values are presented as mean \pm standard deviation.

\subsection{Discussion}

\section{Current findings}

In the present study, BOLD and DCE-MRI were applied to study renal oxygenation and function, respectively, in a murine model of normothermic I/R injury. First, BOLD-MRI revealed a significantly higher $R_{2}^{*}$, indicating lower oxygenation, in the outer medulla of a clamped kidney compared with the contralateral control kidney, even at 24 hours after reperfusion (Figure 8.2). Second, higher $R_{2}^{*}$ values were found in the outer medulla compared with the cortex of the same kidney during I/R injury. These results indicate ongoing ischemic tissue damage in the injured kidney, which might play an important role in the development of ARF in humans after kidney transplantation. In contrast, the cortex of the clamped kidney showed a significantly reduced $R_{2}^{*}$ compared with the control, which might be caused by compensatory mechanisms after I/R injury. Third, ongoing ischemic tissue damage at 24 hours post reperfusion was supported by DCE-MRI measurements of kidney function. Dynamic 
gadolinium enhancement curves showed an increased enhancement of the injured kidney compared with the contralateral control (Figure 8.3). This is likely related to an increased permeability of the renal microvasculature, which was shown to be maximal at 24 hours post reperfusion and can be explained by a disruption of the actin cytoskeleton and loss of adherens junctions between the endothelial cells [271]. The two-compartment filtration model combined with a reference region input function allowed accurate fitting of the DCE-MRI data, which showed a significantly reduced renal function (comprising perfusion and filtration) in the clamped versus the control kidney. Fourth, extensive ischemic injury in the clamped kidney was confirmed by histological analysis (Figure 8.5).

During I/R injury, an increased work load and a consequently increased function might be expected for the contralateral kidney to compensate for the loss of function in the clamped kidney. However, no detectable changes were found for the cortex and outer medulla of the control kidney, as shown by the constant $R_{2}^{*}$ during the entire experiment (Figure 8.2). The presented differences between clamped and control kidneys in these regions are therefore not considered biased by any possible compensatory mechanism of the control kidney.

Taken together, our results demonstrate that both BOLD and DCE-MRI are suitable to study I/R injury in murine kidneys non-invasively. In addition, BOLDMRI uniquely allowed longitudinal evaluation of renal oxygenation and differentiation between the different functional regions of the kidney.

\section{Applicability of MRI}

Although BOLD-MRI can be directly translated into human studies, DCE-MRI relies on the administration of a gadolinium-based low molecular weight contrast agent. These agents were found to be related to an increased risk of nephrogenic systemic fibrosis (NSF) in patients with impaired renal function [272]. However, in the present study, the macrocyclic contrast agent Gadovist was applied, which was shown to be least associated with the development of NSF $[273,274]$ and can also be applied safely in patients with chronic kidney disease (up to stage 4 [275]). It is therefore expected that DCE-MRI with Gadovist can be safely translated into studies regarding $I / R$ injury in humans.

Alternatively, renal perfusion could be measured using arterial spin labeling (ASL). This technique is based on endogenous contrast mechanisms using global and selective inversion pulses. However, the signal-to-noise ratio of ASL images is often low $[276,277]$ and absolute quantification of vascular flow and perfusion can be problematic in patients with large variations in arterial transit times. Furthermore, ASL has been mainly used in the brain and application in the kidneys remains challenging [277]. Although ASL has been applied in transplanted rat kidneys [278], it has not been realized in murine kidneys yet. 
Another non-contrast-enhanced method to study kidney dysfunction is diffusion weighted MR imaging (DWI). The main output parameter of DWI is the apparent diffusion coefficient (ADC), which is a combined measure of capillary perfusion and water diffusion, thereby reflecting the structural integrity of the tissue microenvironment. In animal models of renal I/R injury and transplantation, significantly decreased ADC values were found $[279,280]$. This was likely related to a reduced glomerular filtration rate, tubular cast deposition, and edema of the tubular epithelial cells, which resulted in restricted water diffusion [279]. DWI was also feasible in transplanted kidneys in humans, and provided complementary information to BOLDMRI [255]. Nevertheless, DWI of the kidneys remains technically challenging due to its strong sensitivity to motion (i.e. respiration, peristalsis, and vascular pulsations) and magnetic susceptibility effects at tissue-air interfaces in the abdomen [280]. Clinical solutions include imaging during breath-hold and fast image acquisition using echo-planar imaging techniques. However, echo-planar imaging is precluded in rodent studies due to the strong susceptibility effects, which are even more severe at the high magnetic field strengths typically used in small animal studies [280]. Although these issues can be reduced to some extent using spin-echo acquisition, this is still hampered by bulk motion artifacts, limited spatial resolution, and long acquisition times of at least 30 minutes. DWI was therefore not included in the current MRI protocol.

Isoflurane was shown to have a protective effect on renal I/R injury in rats, via attenuation of the inflammatory response [281]. Although significant differences between clamped and control kidneys were found in the present study, the use of isoflurane anesthesia may have reduced the extent of I/R injury. However, ketamine/xylazine injection anesthesia was not feasible since the duration of anesthesia (maximally 1 hour) is less than the time required for MRI (> 1.5 hours). The potential effect of the type of anesthesia on the BOLD and DCE-MRI results is a topic of future research. Also, future studies investigating the effects of novel therapeutics aimed at reducing renal I/R injury should consider the effect of anesthesia.

\section{Preclinical and clinical perspectives}

BOLD and DCE-MRI are suitable techniques to investigate renal I/R injury noninvasively and longitudinally. Since our methods were developed in mice, they can be directly applied in preclinical studies regarding the development of novel drugs which protect against I/R injury during or after kidney transplantation. In addition, dedicated MRI protocols for murine imaging open the way for studies in transgenic or knock-out animals. This may provide more insight into the effects of various genes in kidney (dys)function and the response to renal I/R injury.

MRI is a highly clinically relevant technique and both BOLD and DCE-MRI can be easily translated into human studies. With respect to the potential risk of NSF it is however advised to monitor serum creatinine levels in DCE-MRI examinations. 
Creatinine was not measured in the current study since the presence of the healthy contralateral kidney ensured a normal global kidney function. In addition, creatinine provides no information on single kidney function.

Besides studying renal I/R injury due to transplantation, the presented MRI methods could also be applied to assess renal injury in patients receiving medication with known renal side-effects, including nephrotoxic xenobiotics [253], cytostatic drugs, or radiation therapy. Regular non-invasive monitoring with BOLD and/or DCE-MRI may allow early detection of renal pathology in these patients, and consequently may lead to an earlier intervention or change of therapy to minimize kidney damage. 


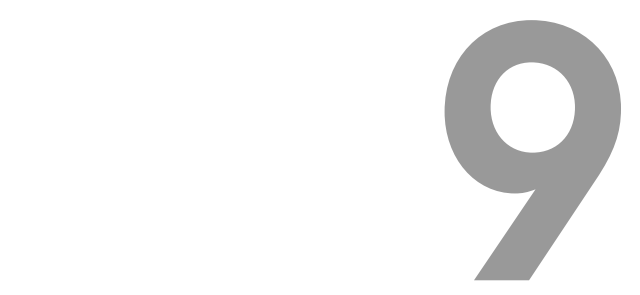

General discussion

Neovascularization plays an important role in many diseases, including cancer and myocardial ischemia due to coronary artery disease. In cancer, angiogenesis provides the growing tumor with its own blood supply, and the level of angiogenic activity is related to tumor malignancy and a consequently poor patient prognosis $[3,4]$. In contrast, angiogenic activity is positively associated with patient prognosis in coronary artery disease $[2,5]$ as it provides a natural repair mechanism through restoration of perfusion, thereby limiting the extent of ischemic tissue damage.

Currently, novel therapeutics are being developed that either inhibit angiogenic activity in cancer [282-285], or stimulate angiogenesis in the heart [218-222]. However, the success of both anti- and pro-angiogenic treatments depends on the availability of clinically relevant in vivo methods to evaluate therapeutic efficacy. Ideally, these methods should meet the following criteria. First, the method should provide an early marker of any therapeutic response (i.e. before macrostructural changes or altered tissue perfusion become apparent). Second, the method should be noninvasive to allow longitudinal monitoring. Third, the method should be quantitative to allow objective comparison of changes in angiogenic activity over time. Fourth, the development of therapeutic neovascularization proceeds in small steps, so any effect should be detectable with high sensitivity.

In this thesis, novel molecular MRI methods were developed to detect and quantify angiogenic activity in preclinical models of cancer and myocardial infarction. In general, molecular MRI is defined as the in vivo detection of biological processes at a molecular or cellular level using MRI techniques. As molecular markers of disease, including upregulation of receptors and adhesion molecules, extracellular matrix degradation, or inflammation, occur relatively early during the angiogenic process, molecular MRI may provide the required early and sensitive (surrogate) biomarker of therapeutic response. 


\subsection{Quantitative MRI in oncology}

In Chapter 3, a novel quantitative in vivo MRI method was developed and applied to selectively image angiogenic activity in subcutaneous solid tumors in mice. Quantum dots were chosen as contrast agent scaffold based on their excellent fluorescent properties, which allowed validation of the MRI results with independent fluorescence microscopy, and their ability to bind multiple gadolinium constructs and targeting ligands. This was especially important as it likely enhanced the contrast agent's relaxivity and targeting efficacy, respectively. Specific targeting to angiogenic vasculature was achieved via coupling of the cNGR ligand, which homes to upregulated CD13 on activated endothelial cells $[136,140,141]$.

Endothelial cell targeting has several distinct advantages over targeting of intraor extracellular markers. First, target binding occurs relatively fast due to the easy accessibility of the targets for intravascular contrast agents. Second, due to the fast binding, there is no need for long circulating contrast agent particles, which would be required for both intra- and extracellular-extravascular targets. A relatively rapid plasma clearance (half life in the order of 10 minutes) is advantageous, as it quickly reduces any signal from unbound circulating agent, which would obscure the signal from bound contrast agent particles. Consequently, images can be obtained within a clinically acceptable time frame after contrast agent injection. Third, reversible binding to vascular targets also results in fast clearance of bound contrast agents, since the agent is directly transported via the blood stream after dissociation from the target and does not have to be reabsorbed into the blood or lymph. Fourth, contrast agent internalization into endosomes was shown to significantly reduce the agent's relaxivity [286]. Fifth, endothelial cell targeting can be achieved using intravenous contrast agent injection, which is considerably less invasive than, for instance, intratumoral injection. A disadvantage of vascular targets, however, is that only $10 \%$ of the cells in a tumor are endothelial [111], which could result in signal reduction due to partial volume effects. In addition, the interaction of the contrast agent and the target needs to be strong enough to overcome shear stress forces at the vascular wall. This is however not considered to be a major problem in tumors, as flow, and therefore shear stress, is much lower in the highly irregular vascular networks in tumors compared with normal tissues.

As shown in Chapter 3, administration of cNGR-labeled quantum dots resulted in a significant increase in $R_{1}$ in the tumor rim compared with unlabeled control quantum dots, despite that the signal increases in the individual $T_{1}$-weighted inversion recovery images were only marginal. This highlights the importance of image quantification. Absolute quantification of $R_{1}$ using multiple images allowed detection of small contrast agent induced changes that would have been missed using visual image analysis. This is especially important when investigating sparse molecular markers of disease. 


\subsection{QUANTITATIVE MRI IN ONCOLOGY}

In Chapter 4 it was shown that quantum dots have magnetic properties, resulting in a reduced signal intensity on spin echo and gradient echo images. Consequently, the contrast enhancement of gadolinium-labeled quantum dots involves both $R_{1}$ and $R_{2}$ effects. Quantitative analysis of changes in tumor relaxation rates after quantum dot injection revealed that although significant changes in $R_{2}$ could be detected, changes in $R_{1}$ were more pronounced and resulted in a higher number of significantly enhanced voxels. $R_{1}$ based methods are therefore more suitable to detect angiogenic activity in tumors using gadolinium-labeled quantum dots.

An important aspect of molecular MRI is validation, as the limited spatial resolution of MRI hampers discrimination between bound, intravascular, or extravascular contrast agent, and between specific and aspecific binding, based on the obtained $\Delta R_{1}$ values and distribution of enhanced tumor regions. Therefore, we applied twophoton laser scanning microscopy (TPLSM) of excised tissue to investigate contrast agent localization at a subcellular resolution. TPLSM showed extensive colocalization of cNGR-labeled quantum dots with vascular endothelial cells in the tumor rim, thereby confirming the MRI results. Additional evidence on the specificity of cNGR was obtained through a competition experiment of cNGR-labeled quantum dots with a large excess of free cNGR (i.e. no paramagnetic or fluorescent label).

The importance of validation is further emphasized in Chapter 6 . Here, MR vessel size imaging was applied to obtain measures related to vessel radius and fractional blood volume over the complete tissue of interest, on a voxel-by-voxel basis. In contrast to molecular MRI, MR vessel size imaging has already been applied in clinical trials [60]. Critical evaluation of the MRI results with ex vivo 3D TPLSM showed that $M R$ vessel size imaging severely overestimates the vessel radius in both tumors and in muscle tissue, whereas it underestimates the relative blood volume. It was therefore concluded that MR vessel size imaging does not allow absolute assessment of vascular morphology. It is, however, suitable for detecting relative changes in vessel radius and blood volume, for instance induced by tumor growth or by anti-angiogenic therapy.

In Chapter 5, molecular MRI and dynamic contrast-enhanced MRI were combined to obtain simultaneous information on tumor perfusion and the level of angiogenic activity. A two-compartment model was developed, which provided insight into the pharmacokinetic behavior of cNGR-labeled and unlabeled quantum dots. Results showed that both targeted and non-targeted contrast agent particles are delivered throughout the entire tumor, and that previously described differences between tumor rim and core can indeed be ascribed to differences in angiogenic activity and are not due to differences in perfusion. When combining therapeutic agents with an imaging label, the developed dynamic molecular MRI method may also allow monitoring of drug delivery over the tumor and other organs. 


\subsection{Quantitative MRI in cardiovascular disease}

Chapter 7 describes the successful application of cNGR-labeled quantum dots to image neovascularization in the murine heart after acute myocardial infarction, using both MRI and TPLSM methods. A strong image contrast was observed in the infarct/border zones of the heart after administration of cNGR-quantum dots, which was absent in mice with myocardial infarction injected with unlabeled quantum dots, or in sham-operated mice injected with cNGR-labeled quantum dots. Validation with TPLSM showed ample colocalization of the CNGR-labeled contrast agent with vascular endothelial cells, indicating effective target binding, whereas unlabeled contrast agent was mostly located in the extravascular space. In addition, TPLSM revealed unique information on microvascular remodeling after myocardial infarction.

A novel method that may complement molecular MRI of the heart is myocardial perfusion imaging. As described in Section 2.4, myocardial perfusion imaging is based on the detection of the first passage of contrast agent through the myocardium, using fast dynamic imaging protocols. Subsequent analysis of the dynamic signal intensity curves using model-independent deconvolution techniques provides quantitative information on local myocardial blood flow [104].

Although it would be scientifically interesting to apply myocardial perfusion imaging in rodents (e.g. in studies with transgenic mice, or in preclinical trials with novel therapies), this is currently unavailable due to the rapid cardiac and respiratory motion of mice and rats. To this extent, we developed a respiratory gated MRI protocol, based on a True-FISP pulse sequence, which allows dynamic imaging of the mouse heart with a temporal resolution of $300 \mathrm{~ms}$ (single slice, one cine frame), and a spatial resolution of $0.6 \times 0.6 \mathrm{~mm}^{2}$. Preliminary results showed accurate detection of the first pass of a gadolinium-based low molecular weight contrast agent in the left ventricular lumen and the myocardium (Figure 9.1). Application of this novel technique in healthy mice and in mice with myocardial infarction, during both rest and pharmacologically induced cardiac stress, will be a topic of future research.

Other cardiovascular applications of quantitative MRI are found in peripheral artery disease. Due to occlusion of one or multiple large arteries in the upper leg, ischemia and consequent angiogenic activity are induced in the lower leg. Additionally, arteriogenesis, i.e. formation of collateral vessels, occurs in the upper leg (see also Section 2.2). Although peripheral artery disease is relatively common in the industrialized society, with an estimated prevalence of 8-12 million in the United States alone, it is often underdiagnosed in routine medical practice [287]. Consequently, there is a need for a reliable (surrogate) biomarker to diagnose peripheral artery disease. Quantitative MRI may provide a potential solution as it allows characterization of peripheral artery disease at different levels. First, vascular/collateral morphology can be depicted over the entire leg using contrast-enhanced MR angiography (Section 2.3). Second, tissue perfusion and blood flow can be estimated using dynamic contrast-enhanced MRI (Section 2.4). Third, it was shown that the $\alpha_{v} \beta_{3}$-integrin is 

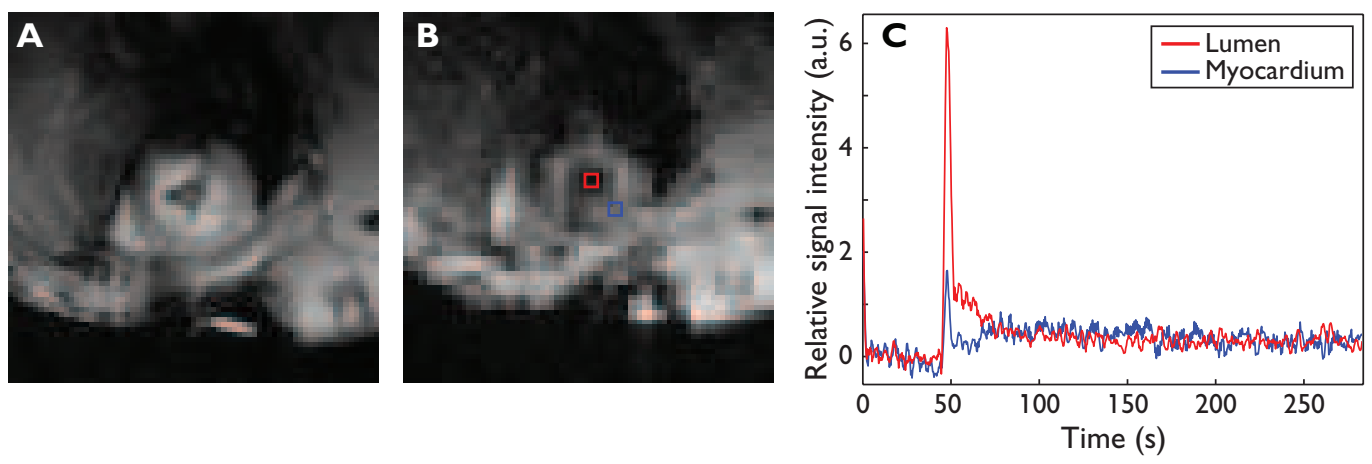

Figure 9.1: A) High resolution (voxel size $0.16 \times 0.16 \mathrm{~mm}^{2}$ ) bright blood short axis image of the mouse heart. B) Myocardial perfusion image showing the location of the regions of interest in the left ventricular lumen (red) and myocardial wall (blue). C). Dynamic signal intensity curves showing first pass of the contrast agent $(0.1 \mathrm{mmol} / \mathrm{kg}$ Gadovist $)$ in the lumen and in the myocardium.

significantly upregulated in both ischemic muscle and on collateral vessels $[288,289]$. This therefore allows molecular MRI of angiogenic activity in the leg using CRGDlabeled contrast agents. Whether angiogenesis in the leg can also be targeted using the CNGR-ligand remains to be shown.

For patients with critical limb ischemia, only limited treatment options are available when revascularization is impossible [290]. Disease progression usually results in amputation, unless there is an intervention to increase arterial perfusion. Similar to coronary artery disease, pro-angiogenic therapy is therefore explored as a novel therapeutic option for these patients. As an example, the phase II, randomized, double-blind, placebo-controlled trial of Lederman et al. showed that intra-arterial administration of recombinant fibroblast growth factor 2 in patients with intermittent claudication resulted in a significant increase in peak walking time at 90 days post therapy [291]. Other pro-angiogenic proteins that have been applied include vascular endothelial growth factor, hepatocyte growth factor, angiopoeitin 1, and hypoxia inducible factor $1 \alpha$ (see references $[290,292]$ for an excellent overview of important clinical trials). The therapeutic end-points used in these trials include transcutaneous oxygen tension, ankle brachial index, walking time, wound healing, and amputation rate [290]. However, these surrogate markers have all been validated for the detection of changes in large vessel perfusion, and not for the accurate assessment of microvascular changes induced by angiogenesis [292]. Novel methods are therefore required and the quantitative methods described in this thesis may provide a basis for the development of molecular MRI techniques to specifically detect angiogenesis in the leg, using either functional or molecular imaging techniques. In addition, MRI allows accurate localization of angiogenic activity within the leg, which is impossible using the aforementioned surrogate end-points. Evaluation of angiogenesis in ischemic hind limb models using molecular MRI will be explored in future research. 


\subsection{Quantitative MRI in nephrology}

A nephrological application of MRI is described in Chapter 8, where quantification of renal oxygenation and function using blood oxygen level dependent (BOLD) and dynamic contrast-enhanced MRI, respectively, were applied to study renal ischemiareperfusion (I/R) injury. I/R injury is an important determinant of acute renal failure and delayed graft function after renal transplantation. Using a murine model of $I / R$ injury, a significant reduction in renal oxygenation and function (comprising perfusion and filtration) was observed, even at 24 hours after reperfusion, indicating ongoing ischemic pathogenesis in the injured kidney. Additionally, the developed methods can be applied to study the potentially beneficial effects of novel treatments aimed at reducing renal I/R injury [251].

An important aspect of the studies described in Chapter 8 is their high clinical relevance and easy translation into the clinic. BOLD-MRI is based on endogenous contrast and can therefore be readily applied in patients with impaired renal function. Dynamic contrast-enhanced MRI requires injection of a gadolinium based contrast agent, which was shown to be associated with an increased risk of developing nephrogenic systemic fibrosis in patients with renal failure [293]. However, in the current study we applied the macrocyclic agent Gadovist, which has the lowest incidence of developing this side effect $[273,274]$, and can also be safely applied in patients with chronic kidney disease (upto stage 4 [275]).

\section{Renal molecular MRI}

An example of molecular MRI in the kidney was recently described by Southworth et al. [294]. Here, renal vascular inflammation in atherosclerotic mice was imaged using a perfluorocarbon nanoparticle contrast agent targeted to endothelial vascular cell adhesion molecule-1 (VCAM-1). Early endothelial activation was found throughout the kidney, particularly in the glomeruli, which occurred prior to any significant change in renal function and structure. Besides increased inflammation, there was also a modest induction of angiogenesis. The methods developed in this thesis might therefore be applied as an adjunctive to VCAM-1 targeting, which might provide more insight into the development of renal inflammation and angiogenesis associated with atherosclerosis.

A second example of renal molecular MRI was provided by Bennet et al. [295], who developed a method to non-invasively detect the structural integrity of the basement membrane in the kidney glomerulus. Instead of using a high affinity ligand, specific targeting was achieved via electrostatic interactions between a cationic contrast agent and the negatively charged proteoglycans of the basement membrane. In a murine model of glomerular basement membrane breakdown, a significantly enhanced contrast was observed using the cationic contrast agent compared with an anionic control contrast agent. As degradation and subsequent reestablishment of 
the basement membrane are also essential steps during angiogenesis (see Section 2.2), combined imaging of basement membrane integrity with the level of activated vascular endothelial cells may provide more insight into the maturity of the neovasculature.

\subsection{Translational challenges in molecular MRI}

Despite the promising results obtained with molecular MRI of angiogenesis described in this thesis, big challenges are still ahead before molecular MRI can be translated into the clinic. First, more sensitive imaging techniques with a higher spatial resolution are required for accurate detection of the spatial extent of angiogenic activity. This can be achieved via faster imaging sequences, using for instance a Look-Locker based approach $[171,296]$ or via more advanced hardware, including cryoprobes and dedicated parallel imaging coils, which allow imaging with higher signal-to-noise ratio without sacrificing imaging time or spatial resolution. Likely, a combination of both approaches will determine the balance between optimal image quality, resolution, and still acceptable acquisition times. Second, the sensitivity and reproducibility of molecular MRI need to be fully characterized. Third, suitable clinical biomarkers are needed which are strongly upregulated during disease and can be targeted with high affinity ligands. Fourth, major improvements are required in contrast agent design, as will be described in more detail in the following section.

\section{Molecular MRI contrast agents}

Clinically acceptable molecular MRI agents need to be cleared from the body, should be non-toxic, should not interfere with the targeted biological process, should bind with high affinity to the target, and should generate sufficient contrast enhancement. Although the quantum dot based contrast agent described in this thesis is a very useful tool for preclinical research, it will never be clinically applicable due to the potential release of toxic cadium and selenium ions, and its strong accumulation in the liver and spleen. In addition, this precludes evaluation of angiogenic activity in these organs. A potentially successful strategy towards clinical translation of molecular MRI is to design low molecular weight gadolinium based agents targeted to a very abundant disease marker, such as fibrin, collagen, or other matrix components $[229,297]$. The small molecular size ensures renal clearance and low toxicity, whereas the strong binding and high target concentration guarantee sufficient signal enhancement. However, for a cellular targeting agent, rough estimations showed that approximately $10^{6}$ contrast agent molecules are still required per cell to obtain sufficient contrast enhancement using a low molecular weight agent containing one or a few gadolinium ions per molecule [298]. Another option might be to apply biodegradable polymers as contrast agent. Their relatively large size results in a high 
relaxivity due to the high gadolinium load and reduced particle tumbling rate. Consequently, the number of required contrast agent particles for high molecular weight contrast agents was shown to be considerably lower compared with low molecular weight agents [298]. Furthermore, biodegradable agents can be cleared via renal filtration after degradation of the polymer [132].

An alternative approach is to use superparamagnetic iron oxide particles instead of gadolinium-based contrast agents for molecular MRI. Iron oxide particles provide sufficient image contrast via their effect on the local magnetic field strength, resulting in a strong reduction in tissue $T_{2}$ and $T_{2}^{*}$, and their relative contrast enhancement efficacy is considerably larger than for gadolinium based particles [298]. Furthermore, iron oxide particles are cleared via normal iron metabolism and were proven to be safe, even in patients with impaired renal function [234]. Clinical applications of iron oxide include pulmonary MR angiography [299], staging of lymph node status in colorectal cancer patients [244], and imaging of macrophage content in carotid artery atherosclerotic lesions [300]. However, a major disadvantage of iron oxide particles is that they induce negative contrast, which can be difficult to distinguish from susceptibility artifacts arising, for instance, from air-tissue interfaces or patient motion.

To overcome these difficulties with negative contrast, several new MR sequences have been developed which allow imaging of iron oxide with positive contrast. Generally, these methods are based on the changed resonance frequency in the vicinity of iron oxide particles, or on the locally altered magnetic field gradient. Currently, we have implemented three positive contrast techniques for the detection of iron oxide particles: white marker imaging [247], fast low angle positive contrast steady state free precession imaging (FLAPS) [301], and susceptibility gradient mapping (SGM) [246]. Using agarose phantoms with iron oxide, a clearly positive signal was observed for all three methods [302] (Figure 9.2).

Next, targeted iron oxide particles were developed to image angiogenic activity in tumors. The positive imaging methods were compared with traditional negative contrast (i.e. $R_{2}^{*}$ increase), to evaluate the merits of positive contrast imaging in vivo. Preliminary results showed that upon injection of cNGR-labeled iron oxide particles, significantly enhanced voxels could be detected in the tumor rim using $\Delta R_{2}^{*}$-mapping and SGM, whereas white marker and FLAPS were not diagnostically useful [302] (Figure 9.3). However, a full quantitative analysis is required to determine which positive or negative contrast technique is best suited to detect tumor angiogenesis in vivo using targeted iron oxide particles.

\section{Biomarker quantification}

An ultimate challenge in molecular MRI is the absolute quantification of biomarker concentrations. Currently, quantification of $\alpha_{v} \beta_{3}$-integrin concentrations with MRI has only been performed in vitro [303]. Although this provided useful information on 

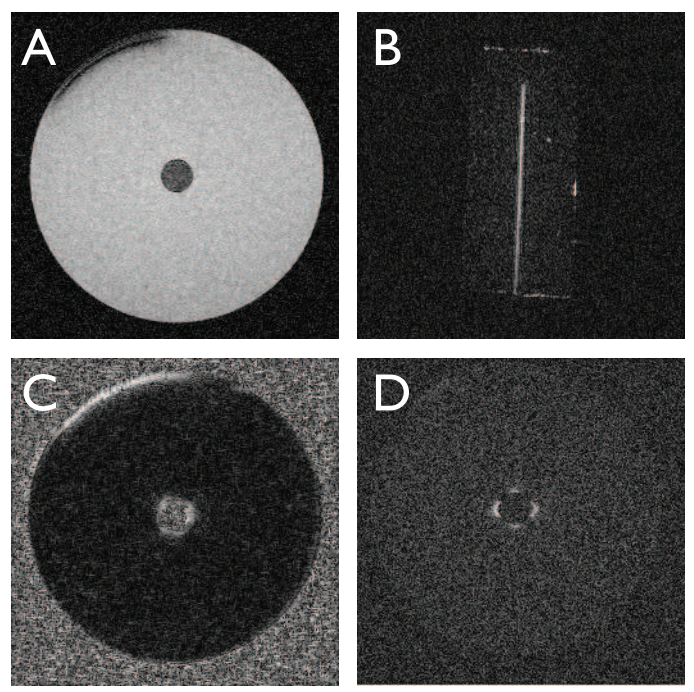

Figure 9.2: Images of $2 \%(\mathrm{w} / \mathrm{v})$ agarose phantom with $3 \mathrm{~mm}$ diameter core doped with iron oxide particles (Fe concentration $0.15 \mathrm{mM}$ ). A) Gradient echo image wit TE $7 \mathrm{~ms}$, TR $750 \mathrm{~ms}$, and $40^{\circ}$ flip angle. B) White marker image with $0 \%$ rephasing gradient, TE $5 \mathrm{~ms}$, TR 750 ms. Note that white marker images cannot be recorded in the coronal plane of the phantom due to directional sensitivity. C) SGM image created from a gradient echo with TE 15 ms and TR 750 ms. D) FLAPS image with a $2^{\circ}$ flip angle, TE 5 ms, and TR 10 ms. Images courtesy of Martijn Wolters.

the angiogenic potential of different tumor cell lines, the presented values likely differ from the in vivo situation due to inherent tumor heterogeneity. Expression levels of $\alpha_{v} \beta_{3}$ in murine tumors have only been determined ex vivo, i.e. after sacrificing the animal, using biochemical methods [304]. Disadvantages of biochemical evaluation of $\alpha_{v} \beta_{3}$ levels are that it is not necessarily related with bioavailability and that $\alpha_{v} \beta_{3}$ expression is not limited to the (neo)vasculature. In vivo biomarker concentrations were estimated to be in the pico- to nanomolar range [305], but accurate values are lacking.

Accurate quantification of biomarker concentration using in vivo molecular MRI requires knowledge of the in vivo contrast agent relaxivities and of the difference in relaxivity between bound and unbound contrast agent, as the detection of both gadolinium- and iron-based contrast agents is based on the indirect relaxation effects of the agent on the surrounding water molecules. Currently, no accurate and validated methods are available to measure this reliably in vivo or in vitro, as discussed in Chapters 4 and 5.

A potential solution towards in vivo biomarker quantification with MRI might be to use ${ }^{19} \mathrm{~F}$ labeled contrast agents or chemical exchange saturation transfer (CEST) agents. The ${ }^{19} \mathrm{~F}$ nucleus has spin $1 / 2$ and has a relative sensitivity that is comparable to protons. ${ }^{19} \mathrm{~F}$ is therefore a suitable alternative nucleus for MRI. CEST agents reduce the water proton signal through transfer of saturated magnetization [306]. 

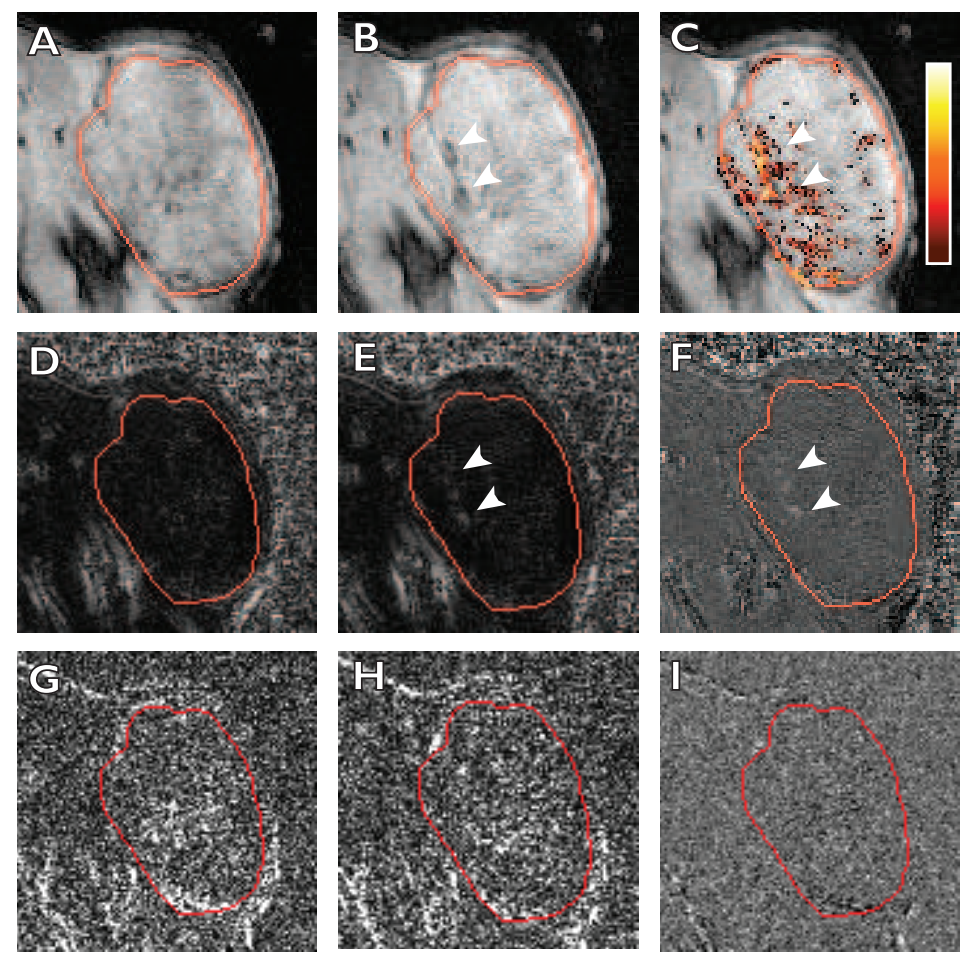

Figure 9.3: Excerpts of axial images of a tumor-bearing mouse injected with cNGR-labeled iron oxide particles. The subcutaneous tumor is outlined in red. Pre (A) and post (B) contrast gradient echo images with TR $750 \mathrm{~ms}$, TE $6 \mathrm{~ms}$, and $40^{\circ}$ flip angle. C) Postcontrast gradient echo with color coded overlay of $\Delta R_{2}^{*}$ (range $0-100 \mathrm{~s}^{-1}$ ). Pre (D) and post (E) contrast SGM images calculated from the images in panel $A$ and $B$, respectively. F) $\triangle \mathrm{SGM}$ image. Pre $(\mathrm{G})$ and post $(\mathrm{H})$ contrast white marker image with $0 \%$ rephasing, TE $5 \mathrm{~ms}$, and TR $75 \mathrm{~ms}$. I) $\triangle$ White marker image. Arrowheads in panels $\mathrm{B}, \mathrm{C}, \mathrm{E}$, and $F$ indicate areas of cNGR-labeled iron oxide accumulation. White marker images showed no contrast agent accumulation. In vivo FLAPS images are not shown due to too many artifacts. Images courtesy of Martijn Wolters.

Specific mobile protons of the CEST agent are saturated by application of selective radio frequency pulses at an agent specific frequency. Subsequent exchange of these protons with the bulk water protons leads to the required signal decrease.

As both ${ }^{19} \mathrm{~F}$ and CEST agents can be detected directly, the post-contrast signal is directly related to contrast agent concentration, and consequently, to biomarker expression levels. A major advantage of both techniques is the absence of any detectable background signal, which simplifies image quantification considerably. Furthermore, both methods theoretically allow imaging of several contrast agents simultaneously, as long as the agents have distinct ${ }^{19} \mathrm{~F}$ resonance frequencies or CEST saturation frequencies $[307,308]$. Consequently, multiple processes can be visualized if the contrast agent is made specific for a certain molecule or organ. In this 
way, unique specificity for a disease process can be introduced, as imaging of only one biological marker (e.g. inflammation) might not be sufficient to differentiate between possible diagnoses. In addition, Waters et al. showed that the signals from bound and unbound ${ }^{19} \mathrm{~F}$ particles can be separated using diffusion-weighted preparation pulses [125]. A disadvantage of both ${ }^{19} \mathrm{~F}$ and CEST imaging is, however, that they require dedicated hardware, which is currently only available in specialized academic centers. Furthermore, high contrast agent concentrations of at least several molars are required to allow accurate detection of both ${ }^{19} \mathrm{~F}$ and CEST agents. For ${ }^{19} \mathrm{~F}$ agents, this could be solved using perfluorocarbon nanoparticles, which have an internal ${ }^{19} \mathrm{~F}$ concentration (i.e. inside each particle) of approximately 100 mo$\operatorname{lar}[309]$.

A final category of novel molecular MRI contrast agents are the so-called smart or activatable agents. These agents function only when cleaved or activated by a certain protein. An elegant example was provided by Shiftan et al., who selectively visualized hyaluronidase activity, an enzyme indirectly involved in tumor angiogenesis, using a gadolinium-based contrast agent covalently attached to the substrate hyaluronan on the surface of agarose beads [310]. Upon cleavage of the substrate, a significant increase in relaxivity was observed and a consequently higher signal was detected. As the increase in signal intensity induced by these smart contrast agents is directly related to biomarker presence, it may provide an alternative towards absolute quantification of biomarker concentrations in vivo.

\subsection{Towards clinical angiogenesis imaging}

As indicated previously, molecular MRI of angiogenesis potentially allows early diagnosis of tumor growth. This is however not expected to become a major future application for the diagnosis of relatively new and small primary and metastasized tumors. Although small tumors of $1-2 \mathrm{~mm}$ in diameter already induce angiogenic activity, the patient experiences no clinical symptoms of tumor growth or metastatization yet and will therefore not visit a physician. In contrast, molecular MRI of tumor angiogenesis is considered useful for the non-invasive staging of previously diagnosed, larger tumors, due to the direct relationship between angiogenic activity and tumor malignancy $[3,4]$. In addition, molecular MRI of tumor angiogenesis could be used for periodic screening in patients with a high familiar risk of developing certain types of cancer. Furthermore, molecular MRI might be applied for the early detection of tumor recurrence.

Non-invasive monitoring of the efficacy of pro- or anti-angiogenic therapies is considered the most important future clinical application of molecular MRI of angiogenesis. In this case, the diagnosis is already confirmed (e.g. histologically) and the radiologist knows what he is looking for. This significantly reduces the complexity of finding the proverbial needle in the hay stack, i.e. the small, local changes in tissue 
angiogenic activity induced by the therapy. Especially for longitudinal assessments, the quantitative methods described in Chapters $3-7$ are expected to become important, as they allow objective comparison between images recorded at different time points. Other potential clinical applications include the development of individualized angiogenic therapy based on assessment of the patient's baseline level of angiogenic activity, and monitoring of targeted drug delivery.

Besides the challenges regarding contrast agent design described in Section 9.4, an important issue in the clinical translation of molecular MRI of angiogenesis is its cost-effectiveness. Currently applied contrast agents are extremely expensive (e.g. the quantum dot contrast agent costs approximately $€ 300$ for one murine dose), and are produced in small batches only. Consequently, significant improvements are required to allow contrast agent synthesis in large quantities and at acceptable costs, before trials in large animal models or in humans will become feasible.

\section{Clinical multimodal imaging}

A development towards the clinical application of multimodal angiogenesis imaging is the introduction of the integrated positron emission tomography (PET)/MRI system [311]. PET/MRI combines the best of both worlds: the high spatial resolution and soft tissue contrast of MRI with the low detection limit for sparse molecular markers of PET. In addition, a hybrid PET/MRI system ensures accurate colocalization of the individual PET and MR images. Compared with molecular MRI, PET molecular imaging requires considerably lower contrast agent doses, which is advantageous for rapid clinical translation. An important potential indication for PET/MRI in oncological imaging includes all tumors where imaging profits from high soft-tissue contrast, i.e. in body regions with complex anatomy. These include musculoskeletal, head/neck, intracranial, breast, and liver tumors [312,313]. For example, in mammography, PET/MRI combines the high sensitivity of MRI to detect breast lesions with the high specificity of PET to differentiate between benign and malignant tumors [312]. Furthermore, MRI can provide valuable, advanced functional and metabolic information using perfusion or diffusion imaging, and MR spectroscopy, respectively, which can complement the molecular information obtained through PET imaging. A specific application of PET/MRI to visualize tumor angiogenesis was presented by Lee et al., who developed a CRGD-labeled probe that can be simultaneously detected using MRI and PET [314]. As an alternative, different probes for MRI and PET could be applied. Molecular processes of disease are complex and it is unlikely that diagnoses can be made using only one molecular biomarker. Consequently, if distinct biomarkers of tumor angiogenesis were selected, this would provide complementary information to that coming from a single-target bimodal probe. As an example, one could target early angiogenic activity using CRGD- or cNGR-labeled contrast agents with one modality, whereas the other modality could be used to image another biomarker, such as NG2. The NG2 proteoglycan is expressed by pericytes 


\subsection{TOWARDS CLINICAL ANGIOGENESIS IMAGING}

in pathological microvasculature, is not detectable in normal quiescent vessels, and can be targeted using small peptide ligands [315-317]. As pericytes are only involved in the later stages of vessel development (Section 2.2), NG2 targeting may provide insight into vessel maturation. The combined approach of two different ligands and imaging modalities may therefore generate novel information on the true angiogenic status of the tissue. Other applications of PET/MR include more accurate differentiation between benign and malignant lesions, improved staging of lymph node status, and more specific assessment of metastatic spread compared with either PET or MRI alone, and compared with its direct competitor PET/CT [312].

In cardiac imaging, PET/MRI may pose several potential benefits over the individual imaging methods as well. Combining left ventricular function as assessed by MRI with PET information on myocardial perfusion and metabolism may improve risk stratification in heart failure [318]. An even more detailed risk assessment could be obtained using myocardial viability as measured with ${ }^{18}$ F-FDG PET and delayed enhancement MRI, which provide information on the extent of the area at risk and the transmurality of irreversibly damaged (scar) tissue, respectively. A specific molecular imaging application of PET/MRI in the heart might be to track iron oxide-labeled stem cell therapy for myocardial infarction with MRI, while simultaneously evaluating the angiogenic response with a PET radiotracer.

However, PET/MRI is presently still experimental and clinical prototypes have only been described for brain imaging [319]. In addition, it is expected that PET/MRI will be more expensive and have a reduced patient throughput compared with MRI alone or with PET/CT. Hicks and Lau therefore proposed that PET/MRI should be designed as a complementary tool running in parallel with PET/CT [313]. In this case, PET/CT should be used for whole-body screening to identify lesions that need more detailed anatomical, functional, and/or molecular characterization with $\mathrm{PET} / \mathrm{MRI}$.

An alternative hybrid system is single photon emission computed tomography (SPECT)/MRI. Compared with PET, SPECT has a two to three orders of magnitude lower sensitivity [320], but it is still considerably more sensitive than MRI. Furthermore, SPECT does not allow absolute quantification of tracer concentration. Distinct advantages of SPECT over PET are lower costs and larger variety in specific radiotracers. In addition, SPECT offers the possibility to widen the observational time window due to the longer half lifes of single photon emitters. Moreover, dual tracer imaging is currently still unique to SPECT, due to the possibility of using multiple energy windows for simultaneous imaging of radiotracers with distinct energies. In PET, dual tracer imaging is more difficult since all tracers have the same energy (511 keV). Although dual tracer imaging could be very beneficial for (pre)clinical molecular imaging of angiogenesis, the development of SPECT/MRI is still in an earlier stage than PET/MRI. Goetz et al. recently described the development of a back-to-back combination of a SPECT imager with a low-field (0.1 T) MRI system [321]. Preliminary results showed accurate colocalization of functional SPECT 
images with anatomical MR imaging in the lungs, kidneys, and brain of mice. These results may provide the basis for the development of a truly intregrated SPECT/MRI system.

\subsection{Conclusions}

In this thesis, the feasibility of non-invasive in vivo molecular MRI of angiogenic activity using a targeted, bimodal contrast agent was demonstrated. Different preclinical models of human disease were applied where angiogenesis plays either a negative or a positive role in patient prognosis, i.e. tumor growth and myocardial infarction, respectively. Both in tumors and in the heart, significantly enhanced tissue contrast could be observed upon intravenous administration of the targeted agent compared with a non-targeted control agent. Results were confirmed by ex vivo three-dimensional fluorescence microscopy techniques. These promising results provide an initial step towards the development of clinical molecular MRI. However, especially in the field of contrast agent design, several challenges have to be faced before molecular MRI can make the step towards the clinical arena and show its added value in medicine. 
Appendices 



\section{A \\ Pharmacokinetic modeling in DCE-MRI}

DCE-MRI data can be analyzed semi-quantitatively using descriptive parameters like initial slope of the curve, maximum peak enhancement, and area-under-the-curve at predefined time-points [322]. However, these parameters depend strongly on the acquisition parameters and contrast administration protocol. Less method dependent measures can be obtained using pharmacokinetic modeling. The most commonly applied pharmacokinetic model is the two-compartment model $[323,324]$. The two compartments are the blood plasma and the extravascular-extracellular space (EES, Figure 2.6). For high quality data, the model allows estimation of the relative plasma volume $\left(v_{p}\right)$, the relative EES volume $\left(v_{e}\right)$, and the volume transfer coefficient $\left(K^{\text {trans }}\right)$. Here, only a brief description of the model will be provided. For more information, we refer to [22,323-326] and references therein.

At any time $t$, the contrast agent concentration in the tumor $C_{T}$ is given by:

$$
C_{T}=K^{\text {trans }} \int_{0}^{t} C_{P}(\tau) e^{-K^{\text {trans }} / v_{e}(t-\tau)} d \tau+v_{p} C_{p}(t)
$$

where $C_{p}(t)$ is the concentration time course in the blood plasma of the tumor (vascular input function, VIF). The concentration $C$ can be derived from the longitudinal relaxation time $T_{1}$ :

$$
\frac{1}{T_{1}(t)}=\frac{1}{T_{10}}+r_{1} \cdot C(t)
$$

with $T_{10}$ being the pre-contrast tissue $T_{1}$ and $r_{1}$ the contrast agent's longitudinal relaxivity. $T_{1}$ can be obtained from the signal intensity of a spoiled gradient recalled echo image using the Ernst formula:

$$
S(t)=S_{0} \frac{\sin \alpha \cdot\left(1-e^{-\mathrm{TR} / T_{1}(t)}\right)}{\left(1-\cos \alpha \cdot e^{-\mathrm{TR} / T_{1}(t)}\right)} \cdot e^{-\mathrm{TE} / T_{2}^{*}}
$$




\section{APPENDIX A. PHARMACOKINETIC MODELING IN DCE-MRI}

in which $S_{0}$ is proportional to the equilibrium magnetization, $\alpha$ is the flip angle, and $T_{2}^{*}$ is the apparent transverse relaxation time. TE and $T R$ are the echo and repetition times, respectively. $S_{0}$ and $T_{10}$ can be determined by recording a series of images with varying flip angles prior to contrast agent injection. For proper $T_{1}$-weighted imaging, the factor $e^{-\mathrm{TE} / T_{2}^{*}}$ in equation $\mathrm{A} .3$ may be ignored when TE is much smaller than $T_{2}^{*}$. Alternative sequences that can be applied to determine tissue $T_{1}$ are inversion recovery, gradient echo, and Look-Locker.

Before $K^{\text {trans }}, v_{e}$, and $v_{p}$ can be extracted by numerical optimization of equation A.1, $C_{P}(t)$ needs to be estimated from the temporal signal in a relatively large vessel adjacent to the tissue of interest using equations $A .2$ and A.3. The plasma $T_{1}$ can be calculated from the measured blood $T_{1}$ using the blood hematocrit Hct:

$$
\frac{1}{T_{1, \text { plasma }}}=\frac{1-\text { Hct }}{T_{1, \text { blood }}}
$$




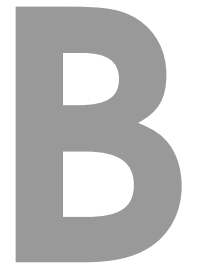

\section{Magnetic effects of quantum dots}

The magnetic effects of non-gadolinium labeled QDs were investigated using the following phantom solutions: $1 \mu \mathrm{M}$ QDs in borate buffer, $0.1 \mathrm{mM}$ gadobutrol (Gadovist) in borate buffer, and borate buffer without any contrast agent. The $R_{1}$ and $R_{2}$ of the samples were measured using IR and MSME sequences as described in Section 4.2. In addition, $R_{2}^{*}$ was determined using a multi-slice multi-echo gradient echo with TR $5000 \mathrm{~ms}$ and TE ranging from 4 to $67 \mathrm{~ms}$ with an echo interval of $7 \mathrm{~ms}$.

The effect of QDs on the relaxation rate was relatively small for $R_{1}$, more pronounced for $R_{2}$, and most prominent for $R_{2}^{*}$ (Table B.1). This is also shown in Figure B.1, where a lower signal intensity is observed for the QD sample in comparison with borate buffer. Taken together, these results demonstrate that QDs have an intrinsic relaxation effect, with an additional (magnetic) signal effect on gradient echo images compared with spin echo images.

Table B.1: Relaxation rates for non-gadolinium labeled QDs, $0.1 \mathrm{mM}$ gadobutrol, and borate buffer at $7 \mathrm{~T}$.

\begin{tabular}{lccr}
\hline & $R_{1}\left(\mathrm{~s}^{-1}\right)$ & $R_{2}\left(\mathrm{~s}^{-1}\right)$ & $R_{2}^{*}\left(\mathrm{~s}^{-1}\right)$ \\
$1 \mu \mathrm{QD}$ & $0.46 \pm 0.00$ & $2.7 \pm 0.1$ & $28.0 \pm 1.3$ \\
$0.1 \mathrm{mM}$ Gadobutrol & $0.93 \pm 0.00$ & $2.7 \pm 0.1$ & $2.9 \pm 0.1$ \\
Borate buffer & $0.42 \pm 0.00$ & $2.0 \pm 0.1$ & $2.5 \pm 0.1$ \\
\hline
\end{tabular}
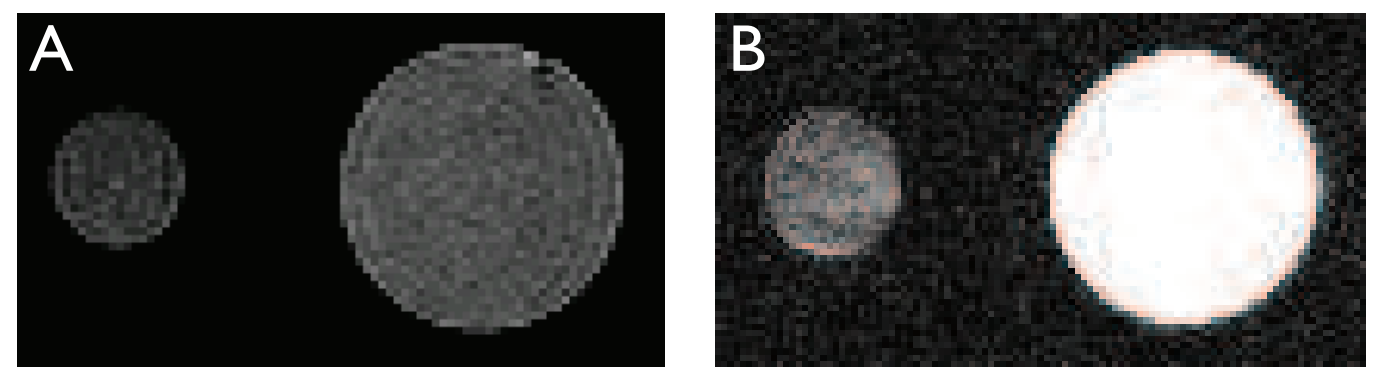

Figure B.1: Spin echo image with TE $100 \mathrm{~ms}$ (A) and gradient echo image with TE $50 \mathrm{~ms}$ (B) showing lower signal intensity for the QD sample $(1 \mu \mathrm{M}$, left) compared with borate buffer (right). Note that the window settings were optimized for each image to allow visualization of the QD sample. 



\section{c}

\section{IR and SE sensitivities}

The signal intensity of a $T_{1}$-weighted inversion recovery (IR) experiment can be described by [153]:

$$
S_{\mathrm{IR}}=S_{\mathrm{IR}}^{0}\left(1-2 e^{-\mathrm{TI} \cdot R_{1}}+e^{-\mathrm{TR} \cdot R_{1}}\right)
$$

where $S_{I R}^{0}$ is a scaling factor including proton density, pre-amplifier gain, and echo time. TI and TR are the inversion and repetition time, respectively, and $R_{1}$ is the longitudinal relaxation rate. The signal intensity of a $T_{2}$-weighted spin echo (SE) sequence is given by [153]:

$$
S_{\mathrm{SE}}=S_{\mathrm{SE}}^{0} \cdot e^{-\mathrm{TE} \cdot R_{2}}
$$

with $S_{\mathrm{SE}}^{0}$ being a scaling factor depending on proton density, pre-amplifier gain, and repetition time. TE is the echo time and $R_{2}$ the transverse relaxation rate.

$R_{1}$ and $R_{2}$ are related to contrast agent concentration $C$ via the relaxivities $r_{1}$ and $r_{2}$, respectively:

$$
R_{1,2}=R_{1,2}^{0}+r_{1,2} \cdot C
$$

where $R_{1,2}^{0}$ represents the intrinsic tissue $R_{1,2}$. Therefore, the sensitivity of a sequence to detect changes in contrast agent concentration is related to the sensitivity to detect changes in relaxation rate. Note that although relaxation rate and contrast agent concentration are related via the relaxivity, the relaxivity is not constant as it was shown to depend on, for instance, magnetic field strength, macromolecular concentration [16], and local conditions of water exchange [17]. Nevertheless, the sensitivities $G_{\mathrm{IR}}$ and $G_{\mathrm{SE}}$ of the IR and SE sequences, respectively, are defined as:

$$
\begin{gathered}
G_{\mathrm{IR}}=\frac{\mathrm{d} S_{\mathrm{IR}}}{\mathrm{d} R_{1}}=S_{\mathrm{IR}}^{0}\left(2 \mathrm{TI} \cdot e^{-\mathrm{TI} \cdot R_{1}}-\mathrm{TR} \cdot e^{-\mathrm{TR} \cdot R_{1}}\right) \\
G_{\mathrm{SE}}=\frac{\mathrm{d} S_{\mathrm{SE}}}{\mathrm{d} R_{2}}=-S_{\mathrm{SE}}^{0} \cdot \mathrm{TE} \cdot e^{-\mathrm{TE} \cdot R_{2}}
\end{gathered}
$$



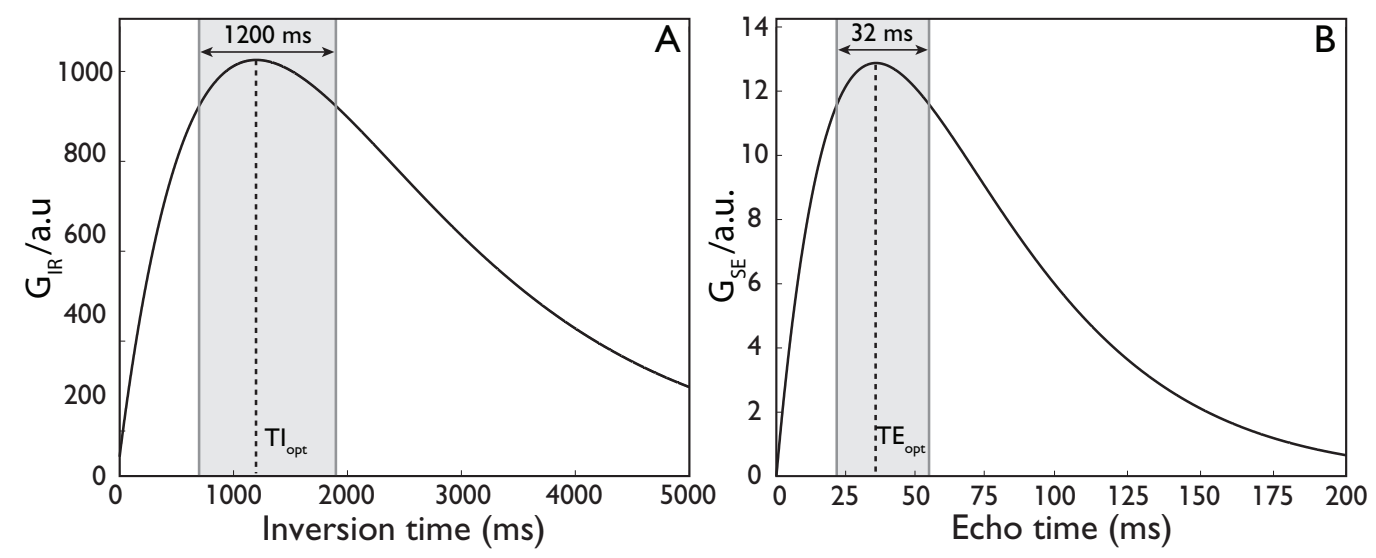

Figure C.1: Relative sensitivities (i.e. $\mathrm{d} S / \mathrm{d} R$ divided by $S_{0}$ ) for a $T_{1}$-weighted inversion recovery (A) and a $T_{2}$-weighted spin echo sequence (B) versus inversion or echo time, respectively. Absolute values are plotted for the spin echo. The maximal sensitivity for IR and SE, obtained at optimal TI and TE respectively, is approximately 80-fold larger for IR. Furthermore, the region where the relative sensitivity is at least $90 \%$ of its maximal value is much wider for IR than for SE (grey shaded areas).

Figure $C .1$ shows a plot of the sensitivities $G_{I R}$ and $G_{S E}$ versus $T I$ and $T E$, respectively. The maximum sensitivity $G_{\max }$ for $\mathrm{IR}$ and $\mathrm{SE}$ is obtained when $\mathrm{TI}=1 / R_{1}$ and $\mathrm{TE}=1 / R_{2}$, respectively. In addition, the maximal change in signal intensity that can be achieved for IR and SE is given by:

$$
\Delta S_{\mathrm{IR}, \mathrm{SE}}^{\max }={\frac{\mathrm{d} S_{\mathrm{IR}, \mathrm{SE}}}{\mathrm{d} R_{1,2}}}^{\max } \cdot \Delta R_{1,2}=G_{\mathrm{IR}, \mathrm{SE}}^{\max } \cdot r_{1,2} \cdot \Delta C
$$




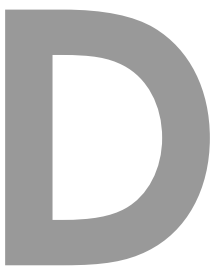

\section{Pharmacokinetic modeling in dynamic molecular MRI}

Within the tumor, the targeted contrast agent can be present in the intravascular space or on the blood vessel wall. Using general compartmental analysis theory [75], the dynamic change in the concentration of bound contrast agent can be described by the differential equation:

$$
\frac{d C_{w}(t)}{d t}=k_{a} C_{p}(t)-k_{d} C_{w}(t)
$$

Here, $C_{w}(t)$ is the concentration of contrast agent bound to the vessel wall, $k_{a}$ and $k_{d}$ are the association and dissociation constants as defined in Figure 5.1B, respectively, and $C_{p}(t)$ is the contrast agent concentration in the blood plasma. Solving Equation D. 1 results in:

$$
C_{w}(t)=k_{a} \int_{0}^{t} C_{p}\left(t^{\prime}\right) e^{-k_{d}\left(t-t^{\prime}\right)} d t^{\prime}
$$

The total tumor contrast agent concentration $C_{T}(t)$ is the sum of the contrast agent concentrations in the blood plasma and vessel wall compartments, weighted by their respective volume fractions $v_{p}$ and $v_{w}$ :

$$
C_{T}(t)=v_{p} C_{p}(t)+v_{w} C_{w}(t)
$$

Combining Equations D.2 and D.3 yields:

$$
C_{T}(t)=v_{p} C_{p}(t)+v_{w} k_{a} \int_{0}^{t} C_{p}\left(t^{\prime}\right) e^{-k_{d}\left(t-t^{\prime}\right)} d t^{\prime}
$$

Using the convolution theorem, Equation D.4 can be rewritten as:

$$
C_{T}(t)=v_{p} C_{p}(t)+C_{p}(t) \otimes H(t)
$$


with the transfer function $H(t)$ given by:

$$
H(t)=K_{a} e^{-k_{d} t}
$$

and the association parameter $K_{a}$ defined as:

$$
K_{a}=v_{w} k_{a}
$$

Note that for non-targeted contrast agents, the mathematical equations described above have the same appearance as the standardized Tofts model (Appendix A, [327]), where $K_{\mathrm{a}}$ and $k_{\mathrm{d}}$ represent the volume transfer constant and the reflux rate, respectively. However, the physiological interpretation here is somewhat different, as there is no exchange between the extracellular-extravascular space and the blood space.

\section{Optimizing the post-contrast imaging time window}

The pharmacokinetic parameters as determined using molecular DCE-MRI can be used directly to estimate the optimal time window for steady-state post-contrast imaging. This would be very beneficial in a clinical setup, since images can be obtained directly after contrast agent injection and there is no need for multiple imaging sessions up to several hours post-contrast.

This concept is further explained in Figure D.1, where the theoretical relative signal intensity is plotted versus the time after contrast agent injection for cNGRpQDs and unlabeled pQDs. The optimal imaging time window is defined as the post-contrast time period during which the relative signal intensity is at least $80 \%$ of its maximum value. For cNGR-pQDs, the optimal imaging time window is between 8 and 23 minutes after contrast agent injection, with a maximum at 13 minutes (Figure D.1).

For clinical imaging one would only be interested in the targeted contrast agent. In preclinical studies, however, targeted agents are often compared with non-targeted controls. Therefore, the optimal preclinical imaging time should be determined based on the difference in relative signal intensity between targeted and non-targeted contrast agents. Figure D.1 demonstrates that the optimal imaging time frame is slightly shifted for the comparison of cNGR-pQDs and unlabeled pQDs, now ranging between 11 and 28 minutes post-contrast, with a maximum at 18 minutes. 


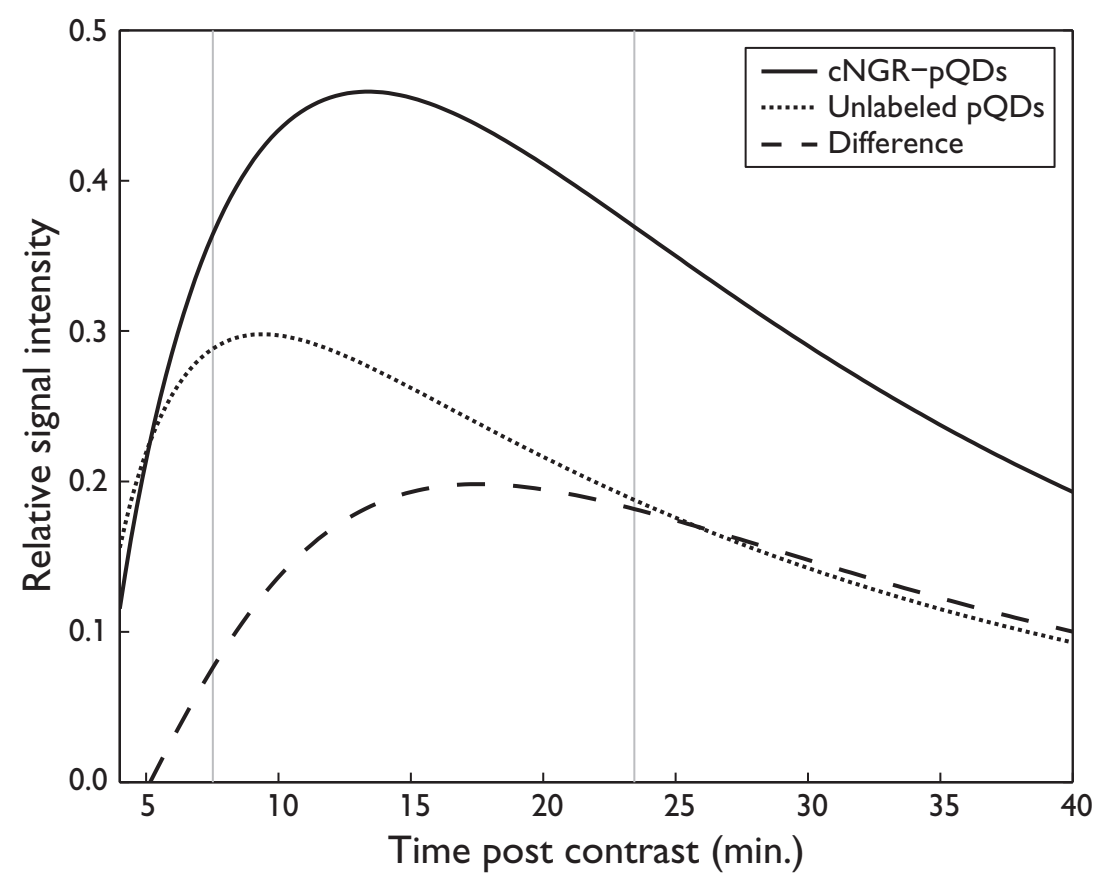

Figure D.1: Calculated relative signal intensity versus time post contrast agent injection for cNGR-pQDs (solid line) and unlabeled pQDs (dotted line). The dashed line represents the difference in relative signal intensity between cNGR-pQDs and unlabeled pQDs. Curves were calculated using the described two-compartment pharmacokinetic model, with the experimentally determined averaged vascular input function and whole tumor $K_{a}, k_{d}$, and $v_{p}$ as input. Vertical grey lines indicate the optimal imaging time window (i.e. relative signal intensity $>80 \%$ of its maximum value) for cNGR-pQDs. Note that the dynamic imaging time of 15 minutes was too short to detect contrast agent wash-out from the tumor in the experiment described in Chapter 5. Therefore, the descending part of the curve is an extrapolation based on the calculated pharmacokinetic parameters. 



\section{References}

[1] Carmeliet P, Jain RK. Angiogenesis in cancer and other diseases. Nature. 2000;407:249-57.

[2] Carmeliet P. Angiogenesis in health and disease. Nat Med. 2003;9:653-60.

[3] Meitar D, Crawford SE, Rademaker AW, Cohn SL. Tumor angiogenesis correlates with metastatic disease, N-myc amplification, and poor outcome in human neuroblastoma. J Clin Oncol. 1996;14:405-14.

[4] Hlatky L, Hahnfeldt P, Folkman J. Clinical application of antiangiogenic therapy: microvessel density, what it does and doesn't tell us. J Natl Cancer. 2002;94:883-93.

[5] Billinger M, Kloos P, Eberli FR, Windecker S, Meier B, Seiler C. Physiologically assessed coronary collateral flow and adverse cardiac ischemic events: a follow-up study in 403 patients with coronary artery disease. J Am Coll Cardiol. 2002;40:1545-50.

[6] Jain RK, Carmeliet PF. Vessels of death or life. Sci Am. 2001;285:38-45.

[7] Ah-See ML, Makris A, Taylor NJ, et al. Early changes in functional dynamic magnetic resonance imaging predict for pathologic response to neoadjuvant chemotherapy in primary breast cancer. Clin Cancer Res. 2008;14:6580-9.

[8] DeLano MC, DeMarco JK. 3.0 T versus 1.5 T MR angiography of the head and neck. Neuroimaging Clin N Am. 2006;16:321-41, xi.

[9] Cappendijk VC, Cleutjens KB, Kessels AG, et al. Assessment of human atherosclerotic carotid plaque components with multisequence MR imaging: initial experience. Radiology. 2005;234:487-92.

[10] Trick GL, Edwards PA, Desai U, Morton PE, Latif Z, Berkowitz BA. MRI retinovascular studies in humans: research in patients with diabetes. NMR Biomed. 2008;21:100312.

[11] Ersoy H, Rybicki FJ. MR angiography of the lower extremities. AJR Am J Roentgenol. 2008;190:1675-84.

[12] Nijveldt R, Beek AM, Hirsch A, Hofman VA, Algra PR, Van Rossum AC. 'No-reflow' after acute myocardial infarction: direct visualisation of microvascular obstruction by gadolinium-enhanced CMR. Neth Heart J. 2008;16:179-81.

[13] Takeuchi M, Matsuzaki K, Uehara H, Nishitani H. Malignant transformation of pelvic endometriosis: MR imaging findings and pathologic correlation. Radiographics. 2006;26:407-17.

[14] Levitt MH. Spin Dynamics: Basics of Nuclear Magnetic Resonance. John Wiley and Sons, Ltd. 1st ed. 2001.

[15] Haacke EM, Brown RW, Thompson MR, Venkatesan R. Magnetic Resonance Imaging Physical Principles and Sequence Design. John Wiley and Sons, Inc. 1st ed. 1999.

[16] Stanisz GJ, Henkelman RM. Gd-DTPA relaxivity depends on macromolecular content. Magn Reson Med. 2000;44:665-7. 


\section{REFERENCES}

[17] Landis CS, Li X, Telang FW, et al. Determination of the MRI contrast agent concentration time course in vivo following bolus injection: effect of equilibrium transcytolemmal water exchange. Magn Reson Med. 2000;44:563-74.

[18] Caravan P, Farrar CT, Frullano L, Uppal R. Influence of molecular parameters and increasing magnetic field strength on relaxivity of gadolinium- and manganese-based T1 contrast agents. Contrast Media Mol Imaging. 2009;4:89-100.

[19] Sipkins DA, Cheresh DA, Kazemi MR, Nevin LM, Bednarski MD, Li KC. Detection of tumor angiogenesis in vivo by alphaVbeta3-targeted magnetic resonance imaging. Nat Med. 1998;4:623-6.

[20] Carmeliet P. Angiogenesis in life, disease and medicine. Nature. 2005;438:932-6.

[21] Esaki J, Marui A, Tabata Y, Komeda M. Controlled release systems of angiogenic growth factors for cardiovascular diseases. Expert Opin Drug Deliv. 2007;4:635-49.

[22] Padhani AR. Dynamic contrast-enhanced MRI in clinical oncology: current status and future directions. J Magn Reson Imaging. 2002;16:407-22.

[23] Neeman M, Dafni H, Bukhari O, Braun RD, Dewhirst MW. In vivo BOLD contrast MRI mapping of subcutaneous vascular function and maturation: validation by intravital microscopy. Magn Reson Med. 2001;45:887-98.

[24] Barrett T, Brechbiel M, Bernardo M, Choyke PL. MRI of tumor angiogenesis. J Magn Reson Imaging. 2007;26:235-49.

[25] Kiessling F, Jugold M, Woenne E C, Brix G. Non-invasive assessment of vessel morphology and function in tumors by magnetic resonance imaging. Eur Radiol. 2007; 17:2136-48.

[26] Gillies RJ, Raghunand N, Karczmar GS, Bhujwalla ZM. MRI of the tumor microenvironment. J Magn Reson Imaging. 2002;16:430-50.

[27] Bhujwalla ZM, Artemov D, Glockner J. Tumor angiogenesis, vascularization, and contrast-enhanced magnetic resonance imaging. Top Magn Reson Imaging. 1999;10:92-103.

[28] Carmeliet P. Mechanisms of angiogenesis and arteriogenesis. Nat Med. 2000;6:38995.

[29] Risau W. Mechanisms of angiogenesis. Nature. 1997;386:671-4.

[30] Murasawa S, Asahara T. Endothelial progenitor cells for vasculogenesis. Physiology. 2005;20:36-42.

[31] Doyle B, Metharom P, Caplice NM. Endothelial progenitor cells. Endothelium. 2006;13:403-10.

[32] Werner N, Nickenig G. Endothelial progenitor cells in health and atherosclerotic disease. Ann Med. 2007;39:82-90.

[33] Lobov IB, Renard RA, Papadopoulos N, et al. Delta-like ligand 4 (DII4) is induced by VEGF as a negative regulator of angiogenic sprouting. Proc Natl Acad Sci USA. 2007;104:3219-24. 
[34] Siekmann AF, Lawson ND. Notch signalling limits angiogenic cell behaviour in developing zebrafish arteries. Nature. 2007;445:781-4.

[35] Folkman J. The role of angiogenesis in tumor growth. Semin Cancer Biol. 1992;3:6571.

[36] Bergers G, Benjamin LE. Tumorigenesis and the angiogenic switch. Nat Rev Cancer. 2003;3:401-10.

[37] Folkman J, Shing Y. Angiogenesis. J Biol Chem. 1992;267:10931-4.

[38] McDonald DM, Choyke PL. Imaging of angiogenesis: from microscope to clinic. Nat Med. 2003;9:713-25.

[39] Scholz D, Cai WJ, Schaper W. Arteriogenesis, a new concept of vascular adaptation in occlusive disease. Angiogenesis. 2001;4:247-57.

[40] Helisch A, Schaper W. Arteriogenesis: the development and growth of collateral arteries. Microcirculation. 2003;10:83-97.

[41] Heil M, Schaper W. Influence of mechanical, cellular, and molecular factors on collateral artery growth (arteriogenesis). Circ Res. 2004;95:449-58.

[42] Prince MR. Gadolinium-enhanced MR aortography. Radiology. 1994;191:155-164.

[43] Ho KY, Leiner T, de Haan MW, van Engelshoven JM. Peripheral MR angiography. Eur Radiol. 1999;9:1765-74.

[44] Zhang H, Maki JH, Prince MR. 3D contrast-enhanced MR angiography. J Magn Reson Imaging. 2007;25:13-25.

[45] Ersoy H, Zhang H, Prince MR. Peripheral MR angiography. J Cardiovasc Magn Reson. 2006;8:517-28.

[46] de Lussanet QG, van Golde JC, Beets-Tan RG, et al. Magnetic resonance angiography of collateral vessel growth in a rabbit femoral artery ligation model. NMR Biomed. 2006;19:77-83.

[47] Nijenhuis RJ, Jacobs MJ, Schurink GW, Kessels AG, Van Engelshoven JM, Backes $\mathrm{WH}$. Magnetic resonance angiography and neuromonitoring to assess spinal cord blood supply in thoracic and thoracoabdominal aortic aneurysm surgery. J Vasc Surg. 2007;45:71-77.

[48] Backes WH, Nijenhuis RJ, Mess WH, Wilmink FA, Schurink GW, Jacobs MJ. Magnetic resonance angiography of collateral blood supply to spinal cord in thoracic and thoracoabdominal aortic aneurysm patients. J Vasc Surg. 2008;48:261-71.

[49] Wang L, Li Z S, Lu J P, Wang F, Liu Q, Tian JM. Cavernous transformation of the portal vein: three-dimensional dynamic contrast-enhanced MR angiography. Abdom Imaging. 2008;33:463-8.

[50] Janssen BJ, Smits JF. Autonomic control of blood pressure in mice: basic physiology and effects of genetic modification. Am J Physiol Regul Integr Comp Physiol. 2002;282:R1545-64.

[51] Brasch R, Pham C, Shames D, et al. Assessing tumor angiogenesis using macromolecular MR imaging contrast media. J Magn Reson Imaging. 1997;7:68-74. 


\section{REFERENCES}

[52] Brasch R, K Turetschek. MRI characterization of tumors and grading angiogenesis using macromolecular contrast media: status report. Eur J Radiol. 2000;34:148-155.

[53] Preda A, van Vliet M, Krestin GP, Brasch RC, van Dijke CF. Magnetic resonance macromolecular agents for monitoring tumor microvessels and angiogenesis inhibition. Invest Radiol. 2006;41:325-31.

[54] Fink C, Kiessling F, Bock M, et al. High-resolution three-dimensional MR angiography of rodent tumors: morphologic characterization of intratumoral vasculature. J Magn Reson Imaging. 2003;18:59-65.

[55] Weidner N. Tumoural vascularity as a prognostic factor in cancer patients: The evidence continues to grow. Journal of Pathology. 1998;184:119-122.

[56] Dennie J, Mandeville JB, Boxerman JL, Packard SD, Rosen BR, Weisskoff RM. NMR imaging of changes in vascular morphology due to tumor angiogenesis. Magn Reson Med. 1998;40:793-9.

[57] Jensen JH, Chandra R. MR imaging of microvasculature. Magn Reson Med. 2000;44:224-30.

[58] Tropres I, Grimault S, Vaeth A, et al. Vessel size imaging. Magn Reson Med. 2001;45:397-408.

[59] Tropres I, Lamalle L, Peoc'h M, et al. In vivo assessment of tumoral angiogenesis. Magn Reson Med. 2004;51:533-541.

[60] Kiselev VG, Strecker R, Ziyeh S, Speck O, Hennig J. Vessel size imaging in humans. Magn Reson Med. 2005;53:553-63.

[61] Robinson SP, Ludwig C, Paulsson J, Ostman A. The effects of tumor-derived plateletderived growth factor on vascular morphology and function in vivo revealed by susceptibility MRI. Int J Cancer. 2007;122:1548-1556.

[62] Wade TP, Kozlowski P. Longitudinal studies of angiogenesis in hormone-dependent Shionogi tumors. Neoplasia. 2007;9:563-8.

[63] Brurberg KG, Benjaminsen IC, Dorum LM, Rofstad EK. Fluctuations in tumor blood perfusion assessed by dynamic contrast-enhanced MRI. Magn Reson Med. 2007;58:473-81.

[64] Zahra MA, Hollingsworth KG, Sala E, Lomas DJ, Tan LT. Dynamic contrast-enhanced MRI as a predictor of tumour response to radiotherapy. Lancet Oncol. 2007;8:63-74.

[65] de Lussanet QG, Backes WH, Griffioen AW, et al. Dynamic contrast-enhanced magnetic resonance imaging of radiation therapy-induced microcirculation changes in rectal cancer. Int J Radiat Oncol Biol Phys. 2005;63:1309-15.

[66] Lankester KJ, Taylor JN, Stirling JJ, et al. Dynamic MRI for imaging tumor microvasculature: comparison of susceptibility and relaxivity techniques in pelvic tumors. $J$ Magn Reson Imaging. 2007;25:796-805.

[67] Kiessling F, Morgenstern B, Zhang C. Contrast agents and applications to assess tumor angiogenesis in vivo by magnetic resonance imaging. Curr Med Chem. 2007;14:77-91. 
[68] Parker GJ, Roberts C, Macdonald A, et al. Experimentally-derived functional form for a population-averaged high-temporal-resolution arterial input function for dynamic contrast-enhanced MRI. Magn Reson Med. 2006;56:993-1000.

[69] Simpson NE, Evelhoch JL. Deuterium NMR tissue perfusion measurements using the tracer uptake approach: II Comparison with microspheres in tumors. Magn Reson Med. 1999;42:240-7.

[70] Yankeelov TE, Luci JJ, Lepage M, et al. Quantitative pharmacokinetic analysis of DCE-MRI data without an arterial input function: a reference region model. Magn Reson Imaging. 2005;23:519-29.

[71] Yankeelov TE, DeBusk LM, Billheimer DD, et al. Repeatability of a reference region model for analysis of murine DCE-MRI data at 7T. J Magn Reson Imaging. 2006;24:1140-7.

[72] Yankeelov TE, Cron GO, Addison CL, et al. Comparison of a reference region model with direct measurement of an AIF in the analysis of DCE-MRI data. Magn Reson Med. 2007;57:353-61.

[73] Bazelaire C, Rofsky NM, Duhamel G, et al. Combined T2* and T1 measurements for improved perfusion and permeability studies in high field using dynamic contrast enhancement. Eur Radiol. 2006;16:2083-91.

[74] Cheng HL. T1 measurement of flowing blood and arterial input function determination for quantitative 3D T1-weighted DCE-MRI. J Magn Reson Imaging. 2007;25:1073-8.

[75] Peters AM, Myers MJ. The peripheral circulation and microvascular solute transfer. in Physiological measurements with radionuclides in clinical practice Oxford University Press Inc. 1998.

[76] de Lussanet QG, Langereis S, Beets-Tan RGH, et al. Dynamic contrast-enhanced MR imaging kinetic parameters and molecular weight of dendritic contrast agents in tumor angiogenesis in mice. Radiology. 2005;235:65-72.

[77] Cosgrove D, Eckersley R, Blomley M, Harvey C. Quantification of blood flow. Eur Radiol. 2001;11:1338-44.

[78] Yuan F, Leunig M, Berk DA, Jain RK. Microvascular permeability of albumin, vascular surface area, and vascular volume measured in human adenocarcinoma LS174T using dorsal chamber in SCID mice. Microvasc Res. 1993;45:269-89.

[79] Prinzen FW, Bassingthwaighte JB. Blood flow distributions by microsphere deposition methods. Cardiovasc Res. 2000;45:13-21.

[80] Lüdemann L, Schmitt B, Podrabsky P, Schnackenburg B, Bock J, Gutberlet M. Usage of the $T 1$ effect of an iron oxide contrast agent in an animal model to quantify myocardial blood flow by MRI. Eur J Radiol. 2007;62:247-56.

[81] de Lussanet QG, Beets-Tan RG, Backes WH, et al. Dynamic contrast-enhanced magnetic resonance imaging at 1.5 Tesla with gadopentetate dimeglumine to assess the angiostatic effects of anginex in mice. Eur J Cancer. 2004;40:1262-8. 


\section{REFERENCES}

[82] Tuncbilek N, Karakas HM, Altaner S. Dynamic MRI in indirect estimation of microvessel density, histologic grade, and prognosis in colorectal adenocarcinomas. Abdom Imaging. 2004;29:166-72.

[83] Schlemmer HP, Merkle J, Grobholz R, et al. Can pre-operative contrast-enhanced dynamic MR imaging for prostate cancer predict microvessel density in prostatectomy specimens? Eur Radiol. 2004;14:309-17.

[84] van Dijke CF, Brasch RC, Roberts TP, et al. Mammary carcinoma model: correlation of macromolecular contrast-enhanced MR imaging characterizations of tumor microvasculature and histologic capillary density. Radiology. 1996;198:813-8.

[85] Buckley DL. Uncertainty in the analysis of tracer kinetics using dynamic contrastenhanced T1-weighted MRI. Magn Reson Med. 2002;47:601-6.

[86] Lopata RG, Backes WH, van den Bosch PP, van Riel NA. On the identifiability of pharmacokinetic parameters in dynamic contrast-enhanced imaging. Magn Reson Med. 2007;58:425-9.

[87] Port RE, Knopp MV, Hoffmann U, Milker-Zabel S, Brix G. Multicompartment analysis of gadolinium chelate kinetics: blood-tissue exchange in mammary tumors as monitored by dynamic MR imaging. J Magn Reson Imaging. 1999;10:233-41.

[88] Zhou R, Pickup S, Yankeelov TE, Springer Jr CS, Glickson JD. Simultaneous measurement of arterial input function and tumor pharmacokinetics in mice by dynamic contrast enhanced imaging: effects of transcytolemmal water exchange. Magn Reson Med. 2004;52:248-57.

[89] Padhani AR, Hayes C, Landau S, Leach MO. Reproducibility of quantitative dynamic MRI of normal human tissues. NMR Biomed. 2002;15:143-53.

[90] Galbraith M, Lodge MA, Taylor NJ, et al. Reproducibility of dynamic contrast-enhanced MRI in human muscle and tumours: comparison of quantitative and semi-quantitative analysis. NMR Biomed. 2002;15:132-42.

[91] Roberts C, Issa B, Stone A, Jackson A, Waterton JC, Parker GJ. Comparative study into the robustness of compartmental modeling and model-free analysis in DCE-MRI studies. J Magn Reson Imaging. 2006;23:554-63.

[92] Daldrup H, Shames DM, Wendland M, et al. Correlation of dynamic contrast-enhanced MR imaging with histologic tumor grade: comparison of macromolecular and smallmolecular contrast media. AJR Am J Roentgenol. 1998;171:941-9.

[93] Radjenovic A, Dall BJ, Ridgway JP, Smith MA. Measurement of pharmacokinetic parameters in histologically graded invasive breast tumours using dynamic contrastenhanced MRI. Br J Radiol. 2008;81:120-8.

[94] Jackson A, O'Connor JP, Parker GJ, Jayson GC. Imaging tumor vascular heterogeneity and angiogenesis using dynamic contrast-enhanced magnetic resonance imaging. Clin Cancer Res. 2007;13:3449-59.

[95] Lehman CD, Gatsonis C, Kuhl CK, et al. MRI evaluation of the contralateral breast in women with recently diagnosed breast cancer. N Engl J Med. 2007;356:1295-303. 
[96] Alonzi R, Padhani AR, Allen C. Dynamic contrast enhanced MRI in prostate cancer. Eur J Radiol. 2007;63:335-50.

[97] Kerwin W, Hooker A, Spilker M, et al. Quantitative magnetic resonance imaging analysis of neovasculature volume in carotid atherosclerotic plaque. Circulation. 2003;107:851-6.

[98] Kerwin WS, O'Brien KD, Ferguson MS, Polissar N, Hatsukami TS, Yuan C. Inflammation in carotid atherosclerotic plaque: a dynamic contrast-enhanced MR imaging study. Radiology. 2006;241:459-68.

[99] Jain RK, Finn AV, Kolodgie FD, Gold HK, Virmani R. Antiangiogenic therapy for normalization of atherosclerotic plaque vasculature: a potential strategy for plaque stabilization. Nat Clin Pract Cardiovasc Med. 2007;4:491-502.

[100] Kerwin WS, Oikawa M, Yuan C, Jarvik GP, Hatsukami TS. MR imaging of adventitial vasa vasorum in carotid atherosclerosis. Magn Reson Med. 2008;59:507-514.

[101] Uren NG, Melin JA, De Bruyne B, Wijns W, Baudhuin T, Camici PG. Relation between myocardial blood flow and the severity of coronary-artery stenosis. N Engl J Med. 1994;330:1782-8.

[102] Gebker R, Schwitter J, Fleck E, Nagel E. How we perform myocardial perfusion with cardiovascular magnetic resonance. J Cardiovasc Magn Reson. 2007;9:539-47.

[103] Kellman P, Arai AE. Imaging sequences for first pass perfusion - a review. J Cardiovasc Magn Reson. 2007;9:525-37.

[104] Jerosch-Herold M, Swingen C, Seethamraju RT. Myocardial blood flow quantification with MRI by model-independent deconvolution. Med Phys. 2002;29:886-97.

[105] Werner GS. Collaterals: how important are they? Heart. 2007;93:778-9.

[106] Muehling OM, Huber A, Cyran C, et al. The delay of contrast arrival in magnetic resonance first-pass perfusion imaging: a novel non-invasive parameter detecting collateraldependent myocardium. Heart. 2007;93:842-7.

[107] Jerosch-Herold M, Hu X, Murthy NS, Seethamraju RT. Time delay for arrival of MR contrast agent in collateral-dependent myocardium. IEEE Trans Med Imaging. 2004;23:881-90.

[108] Massoud TF, Gambhir SS. Molecular imaging in living subjects: seeing fundamental biological processes in a new light. Genes Dev. 2003;17:545-80.

[109] Winter PM, Caruthers SD, Wickline SA, Lanza GM. Molecular imaging by MRI. Curr Cardiol Rep. 2006;8:65-9.

[110] Zhang C, Jugold M, Woenne EC, et al. Specific targeting of tumor angiogenesis by RGD-conjugated ultrasmall superparamagnetic iron oxide particles using a clinical $1.5 T$ magnetic resonance scanner. Cancer Res. 2007;67:1555-62.

[111] Baeten Cl, Wagstaff J, Verhoeven IC, Hillen HF, Griffioen AW. Flow cytometric quantification of tumour endothelial cells; an objective alternative for microvessel density assessment. Br J Cancer. 2002;87:344-7. 


\section{REFERENCES}

[112] Kok RJ, Schraa AJ, Bos EJ, et al. Preparation and functional evaluation of RGDmodified proteins as alpha(v)beta(3) integrin directed therapeutics. Bioconjug Chem. 2002;13:128-35.

[113] Mulder WJ, Strijkers GJ, Habets JW, et al. MR molecular imaging and fluorescence microscopy for identification of activated tumor endothelium using a bimodal lipidic nanoparticle. Faseb J. 2005;19:2008-10.

[114] Strijkers GJ, Mulder WJ, van Heeswijk RB, et al. Relaxivity of liposomal paramagnetic MRI contrast agents. MAGMA. 2005;18:186-192.

[115] Schmieder AH, Winter PM, Caruthers SD, et al. Molecular MR imaging of melanoma angiogenesis with alphanubeta3-targeted paramagnetic nanoparticles. Magn Reson Med. 2005;53:621-7.

[116] Winter PM, Morawski AM, Caruthers SD, et al. Molecular imaging of angiogenesis in early-stage atherosclerosis with alpha(v)beta3-integrin-targeted nanoparticles. Circulation. 2003;108:2270-4.

[117] Anderson SA, Rader RK, Westlin WF, et al. Magnetic resonance contrast enhancement of neovasculature with alpha(v)beta(3)-targeted nanoparticles. Magn Reson Med. 2000;44:433-9.

[118] Schmieder AH, Williams TA, Allen JS, et al. High-Resolution 3D MRI mapping of tumor angiogenesis using a5b1-targeted perfluorocarbon nanoparticles. in Proceedings 15th Scientific Meeting, International Society for Magnetic Resonance in Medicine (Berlin):1187 2007.

[119] Oostendorp M, Douma K, Hackeng TM, et al. Quantitative molecular magnetic resonance imaging of tumor angiogenesis using cNGR-labeled paramagnetic quantum dots. Cancer Res. 2008;68:7676-83.

[120] Morawski AM, Winter PM, Crowder KC, et al. Targeted nanoparticles for quantitative imaging of sparse molecular epitopes with MRI. Magn Reson Med. 2004;51:480-6.

[121] Mulder WJ, Strijkers GJ, Griffioen AW, et al. A liposomal system for contrast-enhanced magnetic resonance imaging of molecular targets. Bioconjug Chem. 2004;15:799-806.

[122] Kang HW, Torres D, Wald L, Weissleder R, Bogdanov Jr AA. Targeted imaging of human endothelial-specific marker in a model of adoptive cell transfer. Lab Invest. 2006;86:599-609.

[123] Sipkins DA, Gijbels K, Tropper FD, Bednarski M, Li KC, Steinman L. ICAM-1 expression in autoimmune encephalitis visualized using magnetic resonance imaging. $J$ Neuroimmunol. 2000;104:1-9.

[124] Geninatti Crich S, Bussolati B, Tei L, et al. Magnetic resonance visualization of tumor angiogenesis by targeting neural cell adhesion molecules with the highly sensitive gadolinium-loaded apoferritin probe. Cancer Res. 2006;66:9196-201.

[125] Waters EA, Chen J, Yang X, et al. Detection of targeted perfluorocarbon nanoparticle binding using 19F diffusion weighted MR spectroscopy. Magn Reson Med. 2008;60:1232-6. 
[126] Langereis S, de Lussanet QG, van Genderen MHP, et al. Evaluation of Gd(III)DTPAterminated poly (propylene imine) dendrimers as contrast agents for MR imaging. NMR in Biomedicine. 2006;19:133-141.

[127] Arbab AS, Pandit SD, Anderson SA, et al. Magnetic resonance imaging and confocal microscopy studies of magnetically labeled endothelial progenitor cells trafficking to sites of tumor angiogenesis. Stem Cells. 2006;24:671-8.

[128] Weber A, Pedrosa I, Kawamoto A, et al. Magnetic resonance mapping of transplanted endothelial progenitor cells for therapeutic neovascularization in ischemic heart disease. Eur J Cardiothorac Surg. 2004;26:137-43.

[129] Partlow KC, Chen J, Brant JA, et al. 19F magnetic resonance imaging for stem/progenitor cell tracking with multiple unique perfluorocarbon nanobeacons. Faseb J. 2007;21:1647-54.

[130] Kanal E, Broome DR, Martin DR, Thomsen HS. Response to the FDA's May 23, 2007, nephrogenic systemic fibrosis update. Radiology. 2008;246:11-4.

[131] Website FDA: information for healthcare professionals gadolinium-based contrast agents for magnetic resonance imaging (marketed as Magnevist, MultiHance, Omniscan, OptiMARK, ProHance). Last updated May 23 2007. Accessed February 15, 2008

[132] Mohs AM, Lu ZR. Gadolinium(III)-based blood-pool contrast agents for magnetic resonance imaging: status and clinical potential. Expert Opin Drug Deliv. 2007;4:14964.

[133] Ladd ME. High-field-strength magnetic resonance: potential and limits. Top Magn Reson Imaging. 2007;18:139-52.

[134] Rinck PA, Muller RN. Field strength and dose dependence of contrast enhancement by gadolinium-based MR contrast agents. Eur Radiol. 1999;9:998-1004.

[135] Folkman J. Tumor angiogenesis: therapeutic implications. N Engl J Med. 1971;285:1182-6.

[136] Arap W, Pasqualini R, Ruoslahti E. Cancer treatment by targeted drug delivery to tumor vasculature in a mouse model. Science. 1998;279:377-80.

[137] Curnis F, Sacchi A, Borgna L, Magni F, Gasparri A, Corti A. Enhancement of tumor necrosis factor alpha antitumor immunotherapeutic properties by targeted delivery to aminopeptidase N (CD13). Nat Biotechnol. 2000;18:1185-90.

[138] Zarovni N, Monaco L, Corti A. Inhibition of tumor growth by intramuscular injection of cDNA encoding tumor necrosis factor alpha coupled to NGR and RGD tumor-homing peptides. Hum Gene Ther. 2004;15:373-82.

[139] van Laarhoven HW, Gambarota G, Heerschap A, et al. Effects of the tumor vasculature targeting agent NGR-TNF on the tumor microenvironment in murine lymphomas. Invest New Drugs. 2006;24:27-36.

[140] Curnis F, Arrigoni G, Sacchi A, et al. Differential binding of drugs containing the NGR motif to CD13 isoforms in tumor vessels, epithelia, and myeloid cells. Cancer Res. 2002;62:867-74. 


\section{REFERENCES}

[141] Pasqualini R, Koivunen E, Kain R, et al. Aminopeptidase N is a receptor for tumorhoming peptides and a target for inhibiting angiogenesis. Cancer Res. 2000;60:722-7.

[142] Rangel R, Sun Y, Guzman-Rojas L, et al. Impaired angiogenesis in aminopeptidase N-null mice. Proc Natl Acad Sci USA. 2007;104:4588-93.

[143] Buehler A, van Zandvoort MAMJ, Stelt BJ, et al. cNGR: a novel homing sequence for CD13/APN targeted molecular imaging of murine cardiac angiogenesis in vivo. Arterioscler Thromb Vasc Biol. 2006;26:2681-7.

[144] von Wallbrunn A, Waldeck J, Holtke C, et al. In vivo optical imaging of CD13/APNexpression in tumor xenografts. J Biomed Opt. 2008;13:011007.

[145] Chan WC, Nie S. Quantum dot bioconjugates for ultrasensitive nonisotopic detection. Science. 1998;281:2016-8.

[146] Michalet X, Pinaud FF, Bentolila LA, et al. Quantum dots for live cells, in vivo imaging, and diagnostics. Science. 2005;307:538-44.

[147] Schnolzer M, Alewood P, Jones A, Alewood D, Kent SB. In situ neutralization in Boc-chemistry solid phase peptide synthesis. Rapid, high yield assembly of difficult sequences. Int J Pept Protein Res. 1992;40:180-93.

[148] Dirksen A, Langereis S, de Waal BFM, van Genderen MHP, Hackeng TM, Meijer EW. A supramolecular approach to multivalent target-specific MRI contrast agents for angiogenesis. Chemical Communications. 2005;22:2811-13.

[149] Dirksen A, Meijer EW, Adriaens W, Hackeng TM. Strategy for the synthesis of multivalent peptide-based nonsymmetric dendrimers by native chemical ligation. Chem Commun. 2006;15:1667-9.

[150] Curnis F, Longhi R, Crippa L, et al. Spontaneous formation of L-isoaspartate and gain of function in fibronectin. Journal of Biological Chemistry. 2006;281:36466-36476.

[151] Maes F, Collignon A, Vandermeulen D, Marchal G, Suetens P. Multimodality image registration by maximization of mutual information. IEEE Trans Med Imaging. 1997;16:187-98.

[152] Rorden C, Brett M. Stereotaxic display of brain lesions. Behav Neurol. 2000;12:191200.

[153] Haacke EM, Brown RW, Thompson MR, Venkatesan R. Introductory signal acquisition methods: free induction decay, spin echoes, inversion recovery and spectroscopy. in Magnetic resonance imaging: physical principles and sequence design John Wiley and Sons, Inc. 1st ed. 1999.

[154] Gambarota G, van Laarhoven HW, Philippens M, et al. Assessment of absolute blood volume in carcinoma by USPIO contrast-enhanced MRI. Magn Reson Imaging. 2006;24:279-86.

[155] Padhani AR. Functional MRI for anticancer therapy assessment. Eur J Cancer. 2002;38:2116-27.

[156] O'Brien PC. Procedures for comparing samples with multiple endpoints. Biometrics. 1984; $40: 1079-87$. 
[157] Läuter J. Exact $t$ and $F$ tests for analyzing studies with multiple endpoints. Biometrics. 1996;52:964-970.

[158] de Lussanet QG, Backes WH, Griffioen AW, van Engelshoven JM, Beets-Tan RG. Gadopentetate dimeglumine versus ultrasmall superparamagnetic iron oxide for dynamic contrast-enhanced MR imaging of tumor angiogenesis in human colon carcinoma in mice. Radiology. 2003;229:429-38.

[159] Manninen A, Koskinen M, Reimann SM, Mottelson B. Magnetic properties of quantum dots and rings. European Physical Journal D. 2001;16:381-385.

[160] Wismer GL, Buxton RB, Rosen BR, et al. Susceptibility induced MR line broadening: applications to brain iron mapping. J Comput Assist Tomogr. 1988;12:259-65.

[161] Yang RS, Chang LW, Wu JP, et al. Persistent tissue kinetics and redistribution of nanoparticles, quantum dot 705, in mice: ICP-MS quantitative assessment. Environ Health Perspect. 2007;115:1339-43.

[162] Prinzen L, Miserus RJ, Dirksen A, et al. Optical and magnetic resonance imaging of cell death and platelet activation using annexin a5-functionalized quantum dots. Nano Lett. 2007;7:93-100.

[163] Cho SJ, Maysinger D, Jain M, Roder B, Hackbarth S, Winnik FM. Long-term exposure to $\mathrm{CdTe}$ quantum dots causes functional impairments in live cells. Langmuir. 2007;23:1974-80.

[164] Yu WW, Chang E, Falkner JC, et al. Forming biocompatible and nonaggregated nanocrystals in water using amphiphilic polymers. J Am Chem Soc. 2007;129:2871-9.

[165] Soo Choi H, Liu W, Misra P, et al. Renal clearance of quantum dots. Nat Biotech. 2007;25:1165-1170.

[166] Zimmer JP, Kim SW, Ohnishi S, Tanaka E, Frangioni JV, Bawendi MG. Size series of small indium arsenide-zinc selenide core-shell nanocrystals and their application to in vivo imaging. J Am Chem Soc. 2006;128:2526-2527.

[167] Vliegen RF, Beets GL, von Meyenfeldt MF, et al. Rectal cancer: MR imaging in local staging-is gadolinium-based contrast material helpful? Radiology. 2005;234:179-88.

[168] Freeman A, Gowland P, Jellineck D, et al. Dynamic T1 studies of gadolinium uptake in brain tumors using LL-EPI. MAGMA. 1994;2:409-412.

[169] Schmitt P, Griswold MA, Jakob PM, et al. Inversion recovery TrueFISP: quantification of T1, T2, and spin density. Magn Reson Med. 2004;51:661-7.

[170] Warntjes JB, Dahlqvist O, Lundberg P. Novel method for rapid, simultaneous T1, T2*, and proton density quantification. Magn Reson Med. 2007;57:528-37.

[171] Messroghli DR, Radjenovic A, Kozerke S, Higgins DM, Sivananthan MU, Ridgway JP. Modified Look-Locker inversion recovery (MOLLI) for high-resolution T1 mapping. Magn Reson Med. 2004;52:141-6.

[172] Weissleder R, Mahmood U. Molecular imaging. Radiology. 2001;219:316-33. 


\section{REFERENCES}

[173] Mulder WJ, Cormode DP, Hak S, Lobatto ME, Silvera S, Fayad ZA. Multimodality nanotracers for cardiovascular applications. Nat Clin Pract Cardiovasc Med. 2008;5 Suppl 2:S103-11.

[174] Bakalova R, Zhelev Z, Aoki I, Kanno I. Designing quantum-dot probes. Nature Photonics. $2007 ; 1: 487-489$.

[175] Fu AH, Gu WW, Larabell C, Alivisatos AP. Semiconductor nanocrystals for biological imaging. Current Opinion in Neurobiology. 2005;15:568-575.

[176] Mulder WJ, Koole R, Brandwijk RJ, et al. Quantum dots with a paramagnetic coating as a bimodal molecular imaging probe. Nano Lett. 2006;6:1-6.

[177] van Schooneveld MM, Vucic E, Koole R, et al. Improved biocompatibility and pharmacokinetics of silica nanoparticles by means of a lipid coating: a multimodality investigation. Nano Lett. 2008;8:2517-25.

[178] van Tilborg GA, Mulder WJ, Chin PT, et al. Annexin A5-conjugated quantum dots with a paramagnetic lipidic coating for the multimodal detection of apoptotic cells. Bioconjug Chem. 2006;17:865-8.

[179] Bottomley PA, Foster TH, Argersinger RE, Pfeifer LM. A review of normal tissue hydrogen NMR relaxation times and relaxation mechanisms from 1-100 MHz: dependence on tissue type, NMR frequency, temperature, species, excision, and age. Med Phys. 1984;11:425-48.

[180] Pintaske J, Martirosian P, Graf H, et al. Relaxivity of Gadopentetate Dimeglumine (Magnevist), Gadobutrol (Gadovist), and Gadobenate Dimeglumine (MultiHance) in human blood plasma at 0.2, 1.5, and 3 Tesla. Invest Radiol. 2006;41:213-21.

[181] Dockery SE, Suddarth SA, Johnson GA. Relaxation measurements at $300 \mathrm{MHz}$ using MR microscopy. Magn Reson Med. 1989;11:182-92.

[182] Streif JU, Hiller KH, Waller C, et al. In vivo assessment of absolute perfusion in the murine skeletal muscle with spin labeling MRI. J Magn Reson Imaging. 2003;17:14752.

[183] Cremillieux Y, Ding S, Dunn JF. High-resolution in vivo measurements of transverse relaxation times in rats at 7 Tesla. Magn Reson Med. 1998;39:285-90.

[184] Guilfoyle DN, Dyakin VV, O'Shea J, Pell GS, Helpern JA. Quantitative measurements of proton spin-lattice (T1) and spin-spin (T2) relaxation times in the mouse brain at 7.0 T. Magn Reson Med. 2003;49:576-80.

[185] Corti A, Curnis F, Arap W, Pasqualini R. The neovasculature homing motif NGR: more than meets the eye. Blood. 2008;112:2628-2635.

[186] Winter PM, Caruthers SD, Kassner A, et al. Molecular imaging of angiogenesis in nascent $\mathrm{Vx}-2$ rabbit tumors using a novel alpha(v)beta3-targeted nanoparticle and 1.5 tesla magnetic resonance imaging. Cancer Res. 2003;63:5838-43.

[187] Guccione S, Li KCP, Bednarski MD. Molecular imaging and therapy directed at the neovasculature in pathologies. leee Engineering in Medicine and Biology Magazine. 2004;23:50-56. 
[188] Villanueva FS. Molecular images of neovascularization - Art for art's sake or form with a function? Circulation. 2005;111:3188-3191.

[189] Di Matteo P, Curnis F, Longhi R, et al. Immunogenic and structural properties of the Asn-Gly-Arg (NGR) tumor neovasculature-homing motif. Mol Immunol. 2006;43:1509-18.

[190] Colombo G, Curnis F, De Mori GM, et al. Structure-activity relationships of linear and cyclic peptides containing the NGR tumor-homing motif. J Biol Chem. 2002;277:47891-7.

[191] Savitsky A, Golay MJE. Smoothing and differentiation of data by simplified least squares procedures. Anal Chem. 1964;36:1627-1639.

[192] Bading JR, Yoo PB, Fissekis JD, Alauddin MM, D'Argenio DZ, Conti PS. Kinetic modeling of 5-fluorouracil anabolism in colorectal adenocarcinoma: a positron emission tomography study in rats. Cancer Res. 2003;63:3667-74.

[193] Beer AJ, Haubner R, Goebel M, et al. Biodistribution and pharmacokinetics of the alphavbeta3-selective tracer 18F-galacto-RGD in cancer patients. J Nucl Med. 2005;46:1333-41.

[194] Kim SJ, Lee JS, Im KC, et al. Kinetic modeling of 3'-deoxy-3'-18F-fluorothymidine for quantitative cell proliferation imaging in subcutaneous tumor models in mice. $\mathrm{J}$ NuCl Med. 2008;49:2057-66.

[195] Neubauer AM, Sim H, Winter PM, et al. Nanoparticle pharmacokinetic profiling in vivo using magnetic resonance imaging. Magn Reson Med. 2008;60:1353-61.

[196] Artemov D, Mori N, Okollie B, Bhujwalla ZM. MR molecular imaging of the Her$2 /$ neu receptor in breast cancer cells using targeted iron oxide nanoparticles. Magn Reson Med. 2003;49:403-8.

[197] Mazooz G, Mehlman T, Lai TS, Greenberg CS, Dewhirst MW, Neeman M. Development of magnetic resonance imaging contrast material for in vivo mapping of tissue transglutaminase activity. Cancer Res. 2005;65:1369-75.

[198] Medarova Z, Rashkovetsky L, Pantazopoulos P, Moore A. Multiparametric monitoring of tumor response to chemotherapy by noninvasive imaging. Cancer Res. 2009;69:1182-9.

[199] Folkman J. Angiogenesis in cancer, vascular, rheumatoid and other disease. Nat Med. 1995;1:27-31.

[200] Prinster A, Pierpaoli C, Turner R, Jezzard P. Simultaneous measurement of DeltaR2 and DeltaR2* in cat brain during hypoxia and hypercapnia. Neuroimage. 1997;6:191200.

[201] Valable S, Lemasson B, Farion R, et al. Assessment of blood volume, vessel size, and the expression of angiogenic factors in two rat glioma models: a longitudinal in vivo and ex vivo study. NMR Biomed. 2008;21:1043-56.

[202] Howe FA, McPhail LD, Griffiths JR, Mclntyre D J, Robinson SP. Vessel size index magnetic resonance imaging to monitor the effect of antivascular treatment in a rodent tumor model. Int J Radiat Oncol Biol Phys. 2008;71:1470-6. 


\section{REFERENCES}

[203] Zwick S, Strecker R, Kiselev V, et al. Assessment of vascular remodeling under antiangiogenic therapy using DCE-MRI and vessel size imaging. J Magn Reson Imaging. 2009;29:1125-1133.

[204] Julien C, Payen JF, Tropres I, et al. Assessment of vascular reactivity in rat brain glioma by measuring regional blood volume during graded hypoxic hypoxia. $\mathrm{Br} J$ Cancer. 2004; $91: 374-80$.

[205] Pathak AP, Schmainda KM, Ward BD, Linderman JR, Rebro KJ, Greene AS. MRderived cerebral blood volume maps: issues regarding histological validation and assessment of tumor angiogenesis. Magn Reson Med. 2001;46:735-47.

[206] Bucher D, Scholz M, Stetter M, Obermayer K, Pfluger HJ. Correction methods for three-dimensional reconstructions from confocal images: I Tissue shrinking and axial scaling. J Neurosci Methods. 2000;100:135-43.

[207] Dorph-Petersen KA, Nyengaard JR, Gundersen HJ. Tissue shrinkage and unbiased stereological estimation of particle number and size. J Microsc. 2001;204:232-46.

[208] Nieuwdorp M, Mooij HL, Kroon J, et al. Endothelial glycocalyx damage coincides with microalbuminuria in type 1 diabetes. Diabetes. 2006;55:1127-32.

[209] Loges S, Clausen H, Reichelt U, et al. Determination of microvessel density by quantitative real-time PCR in esophageal cancer: correlation with histologic methods, angiogenic growth factor expression, and lymph node metastasis. Clin Cancer Res. 2007;13:76-80.

[210] Bos R, van Der Hoeven JJ, van Der Wall E, et al. Biologic correlates of (18)fluorodeoxyglucose uptake in human breast cancer measured by positron emission tomography. J Clin Oncol. 2002;20:379-87.

[211] van der Veldt AA, Hooft L, van Diest PJ, et al. Microvessel density and p53 in detecting cervical cancer by FDG PET in cases of suspected recurrence. Eur J Nucl Med Mol Imaging. 2006;33:1408-16.

[212] Brown EB, Campbell RB, Tsuzuki Y, et al. In vivo measurement of gene expression, angiogenesis and physiological function in tumors using multiphoton laser scanning microscopy. Nat Med. 2001;7:864-8.

[213] Leunig M, Yuan F, Menger MD, et al. Angiogenesis, microvascular architecture, microhemodynamics, and interstitial fluid pressure during early growth of human adenocarcinoma LS174T in SCID mice. Cancer Res. 1992;52:6553-60.

[214] Troprès I, Lamalle L, Farion R, Segebarth C, Remy C. Vessel size imaging using low intravascular contrast agent concentrations. MAGMA. 2004;17:313-6.

[215] Jaffer FA, Nahrendorf M, Sosnovik D, Kelly KA, Aikawa E, Weissleder R. Cellular imaging of inflammation in atherosclerosis using magnetofluorescent nanomaterials. Mol Imaging. 2006;5:85-92.

[216] Von Tiedemann M, Fridberger A, Ulfendahl M, Tomo I, Monvel J. Image adaptive point-spread function estimation and deconvolution for in vivo confocal microscopy. Microsc Res Tech. 2006;69:10-20. 
[217] Jaffer FA, Sosnovik DE, Nahrendorf M, Weissleder R. Molecular imaging of myocardial infarction. J Mol Cell Cardiol. 2006;41:921-33.

[218] Ahn A, Frishman WH, Gutwein A, Passeri J, Nelson M. Therapeutic angiogenesis: a new treatment approach for ischemic heart disease-Part I. Cardiol Rev. 2008;16:16371.

[219] Ahn A, Frishman WH, Gutwein A, Passeri J, Nelson M. Therapeutic angiogenesis: a new treatment approach for ischemic heart disease-Part II. Cardiol Rev. 2008;16:21929.

[220] Molin D, Post MJ. Therapeutic angiogenesis in the heart: protect and serve. Curr Opin Pharmacol. 2007;7:158-63.

[221] Simons M, Bonow RO, Chronos NA, et al. Clinical trials in coronary angiogenesis: issues, problems, consensus: An expert panel summary. Circulation. 2000;102:E7386.

[222] Simons M, Ware JA. Therapeutic angiogenesis in cardiovascular disease. Nat Rev Drug Discov. 2003;2:863-71.

[223] Meoli DF, Sadeghi MM, Krassilnikova S, et al. Noninvasive imaging of myocardial angiogenesis following experimental myocardial infarction. J Clin Invest. 2004;113:168491.

[224] Wagner B, Anton M, Nekolla SG, et al. Noninvasive characterization of myocardial molecular interventions by integrated positron emission tomography and computed tomography. J Am Coll Cardiol. 2006;48:2107-15.

[225] Lutgens E, Daemen MJAP, De Muinck ED, Debets J, Leenders P, Smits JFM. Chronic myocardial infarction in the mouse: cardiac structural and functional changes. Cardiovascular Research. 1999;41:586-593.

[226] Dulce MC, Mostbeck GH, Friese KK, Caputo GR, Higgins CB. Quantification of the left ventricular volumes and function with cine MR imaging: comparison of geometric models with three-dimensional data. Radiology. 1993;188:371-6.

[227] Abramoff MD, Magelhaes PJ, Ram SJ. Image processing with ImageJ. Biophotonics International. 2004;11:36-42.

[228] Sato K, Laham RJ, Pearlman JD, et al. Efficacy of intracoronary versus intravenous FGF-2 in a pig model of chronic myocardial ischemia. Ann Thorac Surg. 2000;70:21138.

[229] Helm PA, Caravan P, French BA, et al. Postinfarction myocardial scarring in mice: molecular MR imaging with use of a collagen-targeting contrast agent. Radiology. 2008;247:788-96.

[230] Ross AJ, Yang Z, Berr SS, et al. Serial MRI evaluation of cardiac structure and function in mice after reperfused myocardial infarction. Magn Reson Med. 2002;47:1158-68.

[231] Stegger L, Heijman E, Schafers KP, Nicolay K, Schafers MA, Strijkers GJ. Quantification of left ventricular volumes and ejection fraction in mice using PET, compared with MRI. J Nucl Med. 2009;50:132-8. 


\section{REFERENCES}

[232] Kim RJ, Chen EL, Lima JA, Judd RM. Myocardial Gd-DTPA kinetics determine MRI contrast enhancement and reflect the extent and severity of myocardial injury after acute reperfused infarction. Circulation. 1996;94:3318-26.

[233] Grass TM, Lurie DI, Coffin JD. Transitional angiogenesis and vascular remodeling during coronary angiogenesis in response to myocardial infarction. Acta Histochem. 2006;108:293-302.

[234] Neuwelt EA, Hamilton BE, Varallyay CG, et al. Ultrasmall superparamagnetic iron oxides (USPIOs): a future alternative magnetic resonance (MR) contrast agent for patients at risk for nephrogenic systemic fibrosis (NSF)? Kidney Int. 2009;75:465-74.

[235] Abdel-Aty H, Simonetti O, Friedrich MG. T2-weighted cardiovascular magnetic resonance imaging. J Magn Reson Imaging. 2007;26:452-9.

[236] Goldfarb JW, Arnold S, Han J. Recent myocardial infarction: assessment with unenhanced T1-weighted MR imaging. Radiology. 2007;245:245-50.

[237] Wacker CM, Hartlep AW, Pfleger S, Schad LR, Ertl G, Bauer WR. Susceptibilitysensitive magnetic resonance imaging detects human myocardium supplied by a stenotic coronary artery without a contrast agent. J Am Coll Cardiol. 2003;41:834-40.

[238] Chen J, Tung CH, Allport JR, Chen S, Weissleder R, Huang PL. Near-infrared fluorescent imaging of matrix metalloproteinase activity after myocardial infarction. Circulation. 2005;111:1800-5.

[239] Su H, Spinale FG, Dobrucki LW, et al. Noninvasive targeted imaging of matrix metalloproteinase activation in a murine model of postinfarction remodeling. Circulation. 2005;112:3157-67.

[240] Nahrendorf M, Hu K, Frantz S, et al. Factor XIII deficiency causes cardiac rupture, impairs wound healing, and aggravates cardiac remodeling in mice with myocardial infarction. Circulation. 2006;113:1196-202.

[241] Hiller KH, Waller C, Nahrendorf M, Bauer WR, Jakob PM. Assessment of cardiovascular apoptosis in the isolated rat heart by magnetic resonance molecular imaging. $\mathrm{Mol}$ Imaging. 2006;5:115-21.

[242] Hofstra L, Liem IH, Dumont EA, et al. Visualisation of cell death in vivo in patients with acute myocardial infarction. Lancet. 2000;356:209-12.

[243] Sosnovik DE, Schellenberger EA, Nahrendorf M, et al. Magnetic resonance imaging of cardiomyocyte apoptosis with a novel magneto-optical nanoparticle. Magn Reson Med. 2005;54:718-24.

[244] Lahaye MJ, Engelen SM, Kessels AG, et al. USPIO-enhanced MR imaging for nodal staging in patients with primary rectal cancer: predictive criteria. Radiology. 2008;246:804-11.

[245] Dowsett DJ, Kenny PA, Johnston RE. Nuclear medicine: radiopharmaceuticals and imaging equipment. in The Physics of Diagnostic Imaging: 469-509 London, UK: Hodder Arnold. 2nd ed. 2006. 
[246] Dahnke H, Liu W, Herzka D, Frank JA, Schaeffter T. Susceptibility gradient mapping (SGM): a new postprocessing method for positive contrast generation applied to superparamagnetic iron oxide particle (SPIO)-labeled cells. Magn Reson Med. 2008;60:595603.

[247] Seppenwoolde JH, Viergever MA, Bakker CJ. Passive tracking exploiting local signal conservation: the white marker phenomenon. Magn Reson Med. 2003;50:784-90.

[248] Brook NR, Waller JR, Nicholson ML. Nonheart-beating kidney donation: current practice and future developments. Kidney Int. 2003;63:1516-29.

[249] Sutton TA, Fisher CJ, Molitoris BA. Microvascular endothelial injury and dysfunction during ischemic acute renal failure. Kidney Int. 2002;62:1539-49.

[250] Brezis M, Rosen S. Hypoxia of the renal medulla-its implications for disease. N Engl J Med. 1995;332:647-55.

[251] Aydin Z, Van Zonneveld AJ, De Fijter JW, Rabelink TJ. New horizons in prevention and treatment of ischaemic injury to kidney transplants. Nephrol Dial Transplant. 2007;22:342-6.

[252] Djamali A, Sadowski EA, Samaniego-Picota M, et al. Noninvasive assessment of early kidney allograft dysfunction by blood oxygen level-dependent magnetic resonance imaging. Transplantation. 2006;82:621-8.

[253] Hofmann L, Simon-Zoula S, Nowak A, et al. BOLD-MRI for the assessment of renal oxygenation in humans: acute effect of nephrotoxic xenobiotics. Kidney Int. 2006;70:144-50.

[254] Prasad PV, Edelman RR, Epstein FH. Noninvasive evaluation of intrarenal oxygenation with BOLD MRI. Circulation. 1996;94:3271-5.

[255] Thoeny HC, Zumstein D, Simon-Zoula S, et al. Functional evaluation of transplanted kidneys with diffusion-weighted and BOLD MR imaging: initial experience. Radiology. 2006;241:812-21.

[256] Tumkur SM, Vu AT, Li LP, Pierchala L, Prasad PV. Evaluation of intra-renal oxygenation during water diuresis: a time-resolved study using BOLD MRI. Kidney Int. 2006;70:139-43.

[257] Li LP, Ji L, Lindsay S, Prasad PV. Evaluation of intrarenal oxygenation in mice by BOLD MRI on a 3.0T human whole-body scanner. J Magn Reson Imaging. 2007;25:635-8.

[258] Li LP, Ji L, Santos EA, Dunkle E, Pierchala L, Prasad PV. Effect of nitric oxide synthase inhibition on intrarenal oxygenation as evaluated by blood oxygenation leveldependent magnetic resonance imaging. Invest Radiol. 2009;44:67-73.

[259] Haacke EM, Brown RW, Thompson MR, Venkatesan R. Magnetic properties of tissue: theory and measurement. in Magnetic resonance imaging: physical principles and sequence design. John Wiley and Sons, Inc. 1st ed. 1999.

[260] Simon-Zoula SC, Hofmann L, Giger A, et al. Non-invasive monitoring of renal oxygenation using BOLD-MRI: a reproducibility study. NMR Biomed. 2006;19:84-9. 


\section{REFERENCES}

[261] Pedersen M, Dissing TH, Morkenborg J, et al. Validation of quantitative BOLD MRI measurements in kidney: application to unilateral ureteral obstruction. Kidney Int. 2005;67:2305-12.

[262] Sadowski EA, Fain SB, Alford SK, et al. Assessment of acute renal transplant rejection with blood oxygen level-dependent MR imaging: initial experience. Radiology. 2005;236:911-9.

[263] Dujardin M, Sourbron S, Luypaert R, Verbeelen D, Stadnik T. Quantification of renal perfusion and function on a voxel-by-voxel basis: a feasibility study. Magn Reson Med. 2005;54:841-9.

[264] Sourbron SP, Michaely HJ, Reiser MF, Schoenberg SO. MRI-measurement of perfusion and glomerular filtration in the human kidney with a separable compartment model. Invest Radiol. 2008;43:40-8.

[265] Michaely HJ, Sourbron S, Dietrich O, Attenberger U, Reiser MF, Schoenberg SO. Functional renal MR imaging: an overview. Abdom Imaging. 2007;32:758-71.

[266] Dujardin M, Luypaert R, Vandenbroucke F, et al. Combined T1-based perfusion MRI and MR angiography in kidney: first experience in normals and pathology. Eur $J$ Radiol. 2009;69:542-9.

[267] Sadick M, Schock D, Kraenzlin B, Gretz N, Schoenberg SO, Michaely HJ. Morphologic and dynamic renal imaging with assessment of glomerular filtration rate in a pcy-mouse model using a clinical 3.0 Tesla scanner. Invest Radiol. 2009.

[268] Sari-Sarraf F, Pomposiello S, Laurent D. Acute impairment of rat renal function by L-NAME as measured using dynamic MRI. MAGMA. 2008;21:291-7.

[269] Faranesh AZ, Yankeelov TE. Incorporating a vascular term into a reference region model for the analysis of DCE-MRI data: a simulation study. Phys Med Biol. 2008:53:2617-31.

[270] Leemans JC, Stokman G, Claessen N, et al. Renal-associated TLR2 mediates ischemia/reperfusion injury in the kidney. J Clin Invest. 2005;115:2894-903.

[271] Sutton TA, Mang HE, Campos SB, Sandoval RM, Yoder MC, Molitoris BA. Injury of the renal microvascular endothelium alters barrier function after ischemia. Am J Physiol Renal Physiol. 2003;285:F191-8.

[272] Agarwal R, Brunelli SM, Williams K, Mitchell MD, Feldman HI, Umscheid CA. Gadolinium-based contrast agents and nephrogenic systemic fibrosis: a systematic review and meta-analysis. Nephrol Dial Transplant. 2009;24:856-63.

[273] Shellock FG, Spinazzi A. MRI safety update 2008: part 1, MRI contrast agents and nephrogenic systemic fibrosis. AJR Am J Roentgenol. 2008;191:1129-39.

[274] Van der Molen AJ. Nephrogenic systemic fibrosis and the role of gadolinium contrast media. J Med Im Rad Oncol. 2008;52:339-50.

[275] Chrysochou C, Buckley DL, Dark P, Cowie A, Kalra PA. Gadolinium-enhanced magnetic resonance imaging for renovascular disease and nephrogenic systemic fibrosis: critical review of the literature and UK experience. J Magn Reson Imaging. 2009;29:887-94. 
[276] Carr JP, Buckley DL, Tessier J, Parker GJ. What levels of precision are achievable for quantification of perfusion and capillary permeability surface area product using ASL? Magn Reson Med. 2007;58:281-9.

[277] Petersen ET, Zimine I, Ho YC, Golay X. Non-invasive measurement of perfusion: a critical review of arterial spin labelling techniques. Br J Radiol. 2006;79:688-701.

[278] Wang JJ, Hendrich KS, Jackson EK, Ildstad ST, Williams DS, Ho C. Perfusion quantitation in transplanted rat kidney by MRI with arterial spin labeling. Kidney Int. 1998;53:1783-91.

[279] Liu AS, Xie JX. Functional evaluation of normothermic ischemia and reperfusion injury in dog kidney by combining MR diffusion-weighted imaging and Gd-DTPA enhanced first-pass perfusion. J Magn Reson Imaging. 2003;17:683-93.

[280] Yang D, Ye Q, Williams DS, Hitchens TK, Ho C. Normal and transplanted rat kidneys: diffusion MR imaging at 7 T. Radiology. 2004;231:702-9.

[281] Lee HT, Ota-Setlik A, Fu Y, Nasr SH, Emala CW. Differential protective effects of volatile anesthetics against renal ischemia-reperfusion injury in vivo. Anesthesiology. 2004;101:1313-24.

[282] Chan A. Antiangiogenic therapy for metastatic breast cancer: current status and future directions. Drugs. 2009;69:167-81.

[283] Norden AD, Drappatz J, Wen PY. Novel anti-angiogenic therapies for malignant gliomas. Lancet Neurol. 2008;7:1152-60.

[284] Seiwert TY, Cohen EE. Targeting angiogenesis in head and neck cancer. Semin Oncol. 2008;35:274-85.

[285] Jain RK. Lessons from multidisciplinary translational trials on anti-angiogenic therapy of cancer. Nat Rev Cancer. 2008;8:309-316.

[286] Kok MB, Hak S, Mulder WJ, Van der Schaft DW, Strijkers GJ, Nicolay K. Cellular compartmentalization of internalized paramagnetic liposomes strongly influences both T1 and T2 relaxivity. Magn Reson Med. 2009;61:1022-32.

[287] Annex BH. Is a simple biomarker for peripheral arterial disease on the horizon? Circulation. 2002;116:1346-8.

[288] Lee $\mathrm{KH}$, Jung $\mathrm{KH}$, Song $\mathrm{SH}$, et al. Radiolabeled RGD uptake and alphav integrin expression is enhanced in ischemic murine hindlimbs. J Nucl Med. 2005;46:472-8.

[289] Fernández B, Broich K. Cell-cell and cell-matrix interactions. in Arteriogenesis. Eds Schaper W and Schaper J. Kluwer Academic Publishers, 2004.

[290] Attanasio S, Snell J. Therapeutic angiogenesis in the management of critical limb ischemia: current concepts and review. Cardiol Rev. 2009;17:115-20.

[291] Lederman RJ, Mendelsohn FO, Anderson RD, et al. Therapeutic angiogenesis with recombinant fibroblast growth factor-2 for intermittent claudication (the TRAFFIC study): a randomised trial. Lancet. 2002;359:2053-8.

[292] Tongers J, Roncalli JG, Losordo DW. Therapeutic angiogenesis for critical limb ischemia: microvascular therapies coming of age. Circulation. 2008;118:9-16. 


\section{REFERENCES}

[293] Broome DR. Nephrogenic systemic fibrosis associated with gadolinium based contrast agents: a summary of the medical literature reporting. Eur J Radiol. 2008;66:230-4.

[294] Southworth R, Kaneda M, Chen J, et al. Renal vascular inflammation induced by Western diet in ApoE-null mice quantified by 19F NMR of VCAM-1 targeted nanobeacons. Nanomedicine. 2009; Epub ahead of print.

[295] Bennett KM, Zhou H, Sumner JP, et al. MRI of the basement membrane using charged nanoparticles as contrast agents. Magn Reson Med. 2008;60:564-74.

[296] Shin W, Gu H, Yang Y. Fast high-resolution T1 mapping using inversion-recovery LookLocker echo-planar imaging at steady state: optimization for accuracy and reliability. Magn Reson Med. 2009;61:899-906.

[297] Botnar RM, Buecker A, Wiethoff AJ, et al. In vivo magnetic resonance imaging of coronary thrombosis using a fibrin-binding molecular magnetic resonance contrast agent. Circulation. 2004;110:1463-6.

[298] Gore JC, Yankeelov TE, Peterson TE, MJ Avison. Molecular imaging without radiopharmaceuticals? J Nucl Med. 2009;50:999-1007.

[299] Ahlström KH, Johansson LO, Rodenburg JB, Ragnarsson AS, Akeson P, Börseth A. Pulmonary MR angiography with ultrasmall superparamagnetic iron oxide particles as a blood pool agent and a navigator echo for respiratory gating: pilot study. Radiology. 1999;211:865-9.

[300] Kooi ME, Cappendijk VC, Cleutjens KB, et al. Accumulation of ultrasmall superparamagnetic particles of iron oxide in human atherosclerotic plaques can be detected by in vivo magnetic resonance imaging. Circulation. 2003;107:2453-8.

[301] Dharmakumar R, Koktzoglou I, Li D. Generating positive contrast from off-resonant spins with steady-state free precession magnetic resonance imaging: theory and proofof-principle experiments. Phys Med Biol. 2006;51:4201-15.

[302] Wolters M. Susceptibility imaging in solid tumors with targeted SPIOs in MRI - in vitro and in vivo comparison of different positive contrast techniques. Master's thesis Eindhoven University of Technology, 2009.

[303] Benedetto S, Pulito R, Crich SG, et al. Quantification of the expression level of integrin receptor alpha(v)beta3 in cell lines and MR imaging with antibody-coated iron oxide particles. Magn Reson Med. 2006;56:711-6.

[304] Zhang X, Xiong Z, Wu Y, et al. Quantitative PET imaging of tumor integrin alphavbeta3 expression with 18F-FRGD. J Nucl Med. 2006;47:113-21.

[305] Nunn AD, Linder KE, Tweedle MF. Can receptors be imaged with MRI agents? Q J Nucl Med. 1997;41:155-62.

[306] Ward KM, Aletras AH, Balaban RS. A new class of contrast agents for MRI based on proton chemical exchange dependent saturation transfer (CEST). J Magn Reson. 2000;143:79-87.

[307] Caruthers SD, Neubauer AM, Hockett FD, et al. In vitro demonstration using 19F magnetic resonance to augment molecular imaging with paramagnetic perfluorocarbon nanoparticles at 1.5 Tesla. Invest Radiol. 2006;41:350-12. 
[308] Ali MM, Liu G, Shah T, Flask CA, Pagel MD. Using two chemical exchange saturation transfer magnetic resonance imaging contrast agents for molecular imaging studies. Acc Chem Res. 2009; Epub ahead of print.

[309] Tran TD, Caruthers SD, Hughes M, et al. Clinical applications of perfluorocarbon nanoparticles for molecular imaging and targeted therapeutics. Int J Nanomedicine. 2007;2:515-26.

[310] Shiftan L, Israely T, Cohen M, et al. Magnetic resonance imaging visualization of hyaluronidase in ovarian carcinoma. Cancer Res. 2005;65:10316-23.

[311] Wehrl HF, Judenhofer MS, Wiehr S, Pichler BJ. Pre-clinical PET/MR: technological advances and new perspectives in biomedical research. Eur J Nucl Med Mol Imaging. 2009;36 Suppl 1:S56-68.

[312] Antoch G, Bockisch A. Combined PET/MRI: a new dimension in whole-body oncology imaging? Eur J Nucl Med Mol Imaging. 2009;36 Suppl 1:S113-20.

[313] Hicks RJ, Lau EWA. PET/MRI: a different spin from under the rim. Eur J Nucl Med Mol Imaging. 2009;36 Suppl 1:S10-4.

[314] Lee HY, Li Z, Chen K, et al. PET/MRI dual-modality tumor imaging using arginineglycine-aspartic (RGD)-conjugated radiolabeled iron oxide nanoparticles. J Nucl Med. 2008;49:1371-9.

[315] Ozerdem U, Grako KA, Dahlin-Huppe K, Monosov E, Stallcup WB. NG2 proteoglycan is expressed exclusively by mural cells during vascular morphogenesis. Dev Dyn. 2001;222:218-27.

[316] Ozerdem U, Monosov E, Stallcup WB. NG2 proteoglycan expression by pericytes in pathological microvasculature. Microvasc Res. 2002;63:129-34.

[317] Burg MA, Pasqualini R, Arap W, Ruoslahti E, Stallcup WB. NG2 proteoglycan-binding peptides target tumor neovasculature. Cancer Res. 1999;59:2869-74.

[318] Nekolla SG, Martinez-Moeller A, Saraste A. PET and MRI in cardiac imaging: from validation studies to integrated applications. Eur J Nucl Med Mol Imaging. 2009;36 Suppl 1:S121-30.

[319] Heiss WD. The potential of PET/MR for brain imaging. Eur J Nucl Med Mol Imaging. 2009;36 Suppl 1:S105-12.

[320] Rahmim A, Zaidi H. PET versus SPECT: strengths, limitations and challenges. NuCl Med Commun. 2008;29:193-207.

[321] Goetz C, Breton E, Choquet P, Israel-Jost V, Constantinesco A. SPECT low-field MRI system for small-animal imaging. J Nucl Med. 2008;49:88-93.

[322] de Vries AF, Griebel J, Kremser C, et al. Tumor microcirculation evaluated by dynamic magnetic resonance imaging predicts therapy outcome for primary rectal carcinoma. Cancer Res. 2001;61:2513-6.

[323] Larsson HB, Stubgaard M, Frederiksen JL, Jensen M, Henriksen O, Paulson OB. Quantitation of blood-brain barrier defect by magnetic resonance imaging and gadoliniumDTPA in patients with multiple sclerosis and brain tumors. Magn Reson Med. 1990;16:117-31. 


\section{REFERENCES}

[324] Tofts PS, Kermode AG. Measurement of the blood-brain barrier permeability and leakage space using dynamic MR imaging. 1. Fundamental concepts. Magn Reson Med. 1991;17:357-67.

[325] Tofts PS. Modeling tracer kinetics in dynamic Gd-DTPA MR imaging. J Magn Reson Imaging. 1997;7:91-101

[326] Choyke PL, Dwyer AJ, Knopp MV. Functional tumor imaging with dynamic contrastenhanced magnetic resonance imaging. J Magn Reson Imaging. 2003;17:590-20.

[327] Tofts PS, Brix G, Buckley DL, et al. Estimating kinetic parameters from dynamic contrast-enhanced $\mathrm{T}(1)$-weighted MRI of a diffusable tracer: standardized quantities and symbols. J Magn Reson Imaging. 1999;10:223-32. 


\section{Summary}

Angiogenesis, the growth of new blood vessels from pre-existing vessels, is involved in many common diseases, where it can play considerably different roles. On the one hand, it is an important negative prognosticator of tumor growth and malignancy. On the other hand, the level of angiogenic activity is positively associated with functional outcome after myocardial infarction. Currently, novel therapies are being developed that either inhibit angiogenesis in cancer, or stimulate angiogenesis after myocardial infarction. However, to fully address their therapeutic efficacy, non-invasive imaging methods are required which provide an early marker of therapeutic response and which can be safely repeated in the same patient to allow longitudinal monitoring.

Magnetic resonance imaging (MRI) is considered the safest and most versatile non-invasive imaging modality available today. It allows anatomic and functional imaging at a superior spatial resolution and soft tissue contrast of almost every region of the human body. In addition, molecular imaging, i.e. the non-invasive in vivo visualization of disease processes at a molecular or cellular level, was shown to be feasible with MRI using specific contrast agents targeted to upregulated biomarkers of disease. As the molecular response to pro- or anti-angiogenic therapy is expected to occur prior to any change in gross anatomy and/or organ function, molecular MRI may provide the required early marker of therapeutic efficacy. Accurate longitudinal evaluation can be achieved via absolute quantification of the therapeutic response in terms that are independent of experimental MRI parameters or physical condition of the patient.

In this thesis, several studies are presented that investigate the microvasculature using quantitative (molecular) MRI techniques. The main focus of this research project was on data acquisition and on the development of novel, quantitative post-processing techniques. The applicability of these methods to explore the microvasculature was demonstrated in preclinical models of tumor growth, myocardial infarction, and ischemia-reperfusion injury in the kidney.

Chapter 1 gives an outline of this thesis and provides a general introduction into the physical basics of MRI and the microvasculature.

In Chapter 2, a thorough review is presented on quantitative contrastenhanced MRI techniques that can be applied to study vessel growth and function. This chapter provides the technical basis for the experimental studies described in the subsequent chapters. 
Chapter 3 describes the successful application of a bimodal (i.e. containing magnetic and fluorescent properties) contrast agent to visualize angiogenic activity in subcutaneous tumors in mice using molecular MRI. Selective contrast agent homing to the angiogenic neovasculature was achieved via a small peptide ligand coupled to the contrast agent surface. Changes in image contrast induced by the contrast agent were most pronounced in the tumor periphery, the tumor area with the known highest level of angiogenic activity. Validation of the MRI results with fluorescence microscopy showed accurate colocalization of the contrast agent with vascular endothelial cells in the tumor rim.

The magnetic properties of the bimodal contrast agent were further explored in Chapter 4. The contrast agent was found to influence both longitudinal and transverse relaxation rates of the surrounding tissue, and the most efficient method of contrast enhancement is therefore a priori unknown. Using absolute quantification of the level and spatial extent of contrast enhancement in murine tumors, it was found that contrast agent induced changes in image contrast can be most accurately detected using techniques based on the longitudinal relaxation rate. Consequently, these methods are likely most suitable for the visualization of angiogenic activity in tumors.

Molecular MRI contrast agents are often relatively large particles with a diameter in the order of several tens to hundreds of nanometers. Combined with the highly irregular tumor microvasculature, this might hamper uniform contrast agent delivery throughout the entire tumor. In addition, the pharmacokinetic behavior of molecular MRI contrast agents is largely unknown. In Chapter 5, a dynamic MRI acquisition method combined with a twocompartment pharmacokinetic model is presented to evaluate contrast agent pharmacokinetics in molecular MRI. The targeted contrast agent was found to have a stronger association with tumor vascular endothelial cells compared with a non-targeted control agent, whereas contrast agent dissociation was weaker. Furthermore, contrast agent delivery could be detected throughout the tumor.

In Chapter 6, MR vessel size imaging was applied to obtain quantitative measures related to the local vessel radius and fractional blood volume in tumors. Although MR vessel size imaging has already been applied in several preclinical as well as in clinical studies, the results were not yet validated with an independent three-dimensional microscopy technique. Comparison of in vivo MR vessel size imaging with ex vivo fluorescence microscopy revealed that MR vessel size imaging severely overestimated vessel radius, whereas it underestimated the relative blood volume. MR vessel size imaging is therefore 
not suitable for absolute quantification of vascular morphology. However, it can be used to detect relative differences, for instance between various tissue types within the same patient, or in one tissue before and after therapy.

Molecular MRI of angiogenic activity in the murine heart after myocardial infarction was explored in Chapter 7. Administration of the targeted bimodal contrast agent resulted in a significantly enhanced image contrast in the infarct and border zones of the heart, which was absent in mice injected with control contrast agent, and in sham-operated mice injected with the targeted agent. The MRI results were confirmed by fluorescence microscopy, which showed extensive colocalization of the targeted contrast agent with the microvasculature in the infarcted area and border zone of the heart.

In Chapter 8, quantitative MRI was applied to study microvascular damage in the kidney after ischemia-reperfusion injury, which likely plays an important role in allograft dysfunction after kidney transplantation. A significant reduction in renal oxygenation and function was found in the injured kidney compared with the contralateral control, which persisted up to 24 hours after reperfusion. This may indicate ongoing ischemic damage to the injured kidney.

Finally, Chapter 9 integrates the results of the various studies and discusses the remaining challenges towards clinical application of quantitative contrast-enhanced MRI of the microvasculature. 



\section{Samenvatting}

Angiogenese, de groei van nieuwe bloedvaten uit reeds bestaande vaten, speelt een belangrijke rol bij een aantal veel voorkomende ziektes. De exacte bijdrage van angiogenese kan zeer verschillend zijn per ziekte. Aan de ene kant is het een belangrijke negatieve voorspeller van tumorgroei en de vorming van uitzaaiingen. Aan de andere kant is de hoeveelheid angiogene activiteit juist geassocieerd met een positieve prognose en (gedeeltelijk) functieherstel na een hartinfarct. Momenteel worden nieuwe therapieën ontwikkeld waarmee angiogenese geremd kan worden bij kwaadaardige tumoren, of waarme angiogenese gestimuleerd kan worden in het geval van een hartinfarct. Echter, om de werkzaamheid van deze therapieën volledig in kaart te kunnen brengen, zijn niet-invasieve technieken, waarmee de therapeutische effecten in een vroeg stadium gedetecteerd kunnen worden, noodzakelijk. Verder moeten de metingen veilig herhaald kunnen worden in dezelfde patiënt, zodat de werkzaamheid van de therapie ook over een langere periode in de tijd kan worden vervolgd.

Beeldvorming door middel van magnetische kernspin resonantie (in het Engels magnetic resonance imaging, afgekort MRI) wordt gezien als de meest veilige en veelzijdige niet-invasieve beeldvormende techniek van de huidige radiologische praktijk. Met MRI kan zowel anatomische als functionele informatie worden verkregen over vrijwel elk orgaan in het menselijk lichaam, met een zeer groot oplossend vermogen en contrast tussen de verschillende weefsels. Verder zijn recentelijk ook moleculaire MRI technieken geïntroduceerd, waarmee ziekteprocessen niet-invasief kunnen worden afgebeeld in levende organismen op moleculair en cellulair niveau. Hiervoor wordt gebruik gemaakt van speciale contrastmiddelen, die met hoge affiniteit binden aan bepaalde biomarkers die verhoogd tot expressie komen tijdens ziekte. Moleculaire MRI wordt gezien als een potentiële vroege marker van therapeutische werkzaamheid, aangezien de effecten van pro- of anti-angiogene therapie zich hoogstwaarschijnlijk het eerst op moleculair niveau zullen manifesteren, voordat veranderingen zichtbaar worden in anatomie en/of orgaanfunctie. Nauwkeurige longitudinale evaluatie kan worden gerealiseerd door middel van absolute kwantificatie van de therapierespons in termen die onafhankelijk zijn van experimentele MRI parameters of van de fysieke conditie van de patiënt.

In dit proefschrift wordt een aantal studies beschreven waarin de microvasculatuur werd onderzocht met kwantitatieve (moleculaire) MRI technieken. Het onderzoeksproject was met name gericht op de data-acquisitie en op de ontwikkeling van nieuwe technieken voor kwantitatieve beeldanalyse. De ont- 
wikkelde technieken werden gebruikt om de microvasculatuur te bestuderen in preklinische (d.w.z. proefdier) modellen van tumorgroei, hartinfarcten en ischemie-reperfusie schade in de nieren.

Hoofdstuk 1 beschrijft kort de inhoud van dit proefschrift en geeft tevens een algemene introductie over de fysische achtergrond van MRI en over de microvasculatuur.

In Hoofdstuk 2 wordt een uitgebreid overzicht gegeven van kwantitatieve contrast-versterkte MR technieken waarmee vaatgroei en -functie bestudeerd kunnen worden. Dit hoofdstuk vormt een actueel literatuuroverzicht en is tevens de technische basis voor de in de daaropvolgende hoofdstukken beschreven experimentele studies.

Hoofdstuk 3 beschrijft de succesvolle toepassing van een nieuw, bimodaal (d.w.z. magnetische en fluorescente eigenschappen bevattend) contrastmiddel voor het afbeelden van angiogene activiteit in onderhuidse tumoren bij muizen, met behulp van moleculaire MRI. Selectieve binding van het contrastmiddel aan de angiogene bloedvaten werd geïntroduceerd door een klein, specifiek peptide aan het contrastmiddel te koppelen. De door het contrastmiddel geïnduceerde veranderingen in beeldcontrast waren voornamelijk gelokaliseerd in de rand van de tumor. Dit tumorgebied staat bekend om zijn hoge mate van angiogene activiteit. Validatie van de MRI resultaten met fluorescentie microscopie toonde aan dat het contrastmiddel specifiek geassocieerd was met de vasculaire endotheelcellen in de rand van het tumorweefsel.

De magnetische eigenschappen van het bimodale contrastmiddel werden verder onderzocht in Hoofdstuk 4. Het contrastmiddel bleek zowel de longitudinale als de transversale relaxatiesnelheden te beïnvloeden, waardoor het a priori onbekend is welke contrast-versterkte MRI techniek het meest optimaal is. Door middel van absolute kwantificatie van het niveau en de ruimtelijke uitgebreidheid van de contrastverandering in tumoren, werd ontdekt dat technieken welke gebaseerd zijn op de longitudinale relaxatiesnelheid het meest geschikt zijn om contrastveranderingen ten gevolge van het bimodale contrastmiddel te kunnen detecteren. Deze methodes zijn daarom waarschijnlijk ook het meest geschikt om angiogene activiteit in tumoren te detecteren met MRI.

Over het algemeen zijn contrastmiddelen voor moleculaire MRI relatief grote deeltjes met een diameter tussen enkele tientallen en honderden nanometers. In combinatie met de sterk onregelmatige stuctuur van de microvaten in de tumor, hindert dit mogelijk de gelijkmatige verdeling van het contrastmiddel over de gehele tumor. Daarnaast is er tot nu toe weinig bekend over 
de farmacokinetische eigenschappen van deze grote moleculaire MRI contrastmiddelen. Hoofdstuk $\mathbf{5}$ beschijft de ontwikkeling van een dynamische MRI methode en een twee-compartimenten farmacokinetisch model, waarmee de farmacokinetiek van moleculaire MRI contrastmiddelen kan worden bestudeerd. In vergelijking met een controle contrastmiddel werd een verhoogde associatie gevonden van het specifieke bimodale contrastmiddel met de vasculaire endotheelcellen, terwijl de dissociatie juist lager was. Verder werd onomstotelijk vastgesteld dat het moleculaire MRI contrastmiddel zich ondanks zijn grootte over de hele tumor kan verspreiden.

In Hoofdstuk $\mathbf{6}$ werd een MRI techniek gebruikt waarmee kwantitatieve informatie kan worden verkregen over de lokale vaatgrootte en het relatieve bloedvolume in een tumor. Ondanks dat deze techniek al in meerdere preklinische en zelfs in klinische studies is toegepast, zijn de resultaten nog niet eerder gevalideerd met een onafhankelijke driedimensionale microscopie techniek. Vergelijking van de in vivo MRI resultaten met ex vivo fluorescentie microscopie toonde aan dat MRI de vaatdiameter aanzienlijk overschat, terwijl het relatieve bloedvolume juist wordt onderschat. Deze MRI techniek is daarom niet geschikt voor de absolute kwantificatie van vasculaire morfologie. Het zou echter wel gebruikt kunnen worden om relatieve verschillen te detecteren, bijvoorbeeld tussen verschillende weefsels in dezelfde patiënt, of binnen één weefseltype voor en na therapie.

Hoofdstuk 7 beschrijft de toepassing van moleculaire MRI voor de visualisatie van angiogene activiteit in het hart, na inductie van een hartinfarct in muizen. Na toediening van het specifieke bimodale contrastmiddel werd een significante verandering in beeldcontrast waargenomen in het infarctgebied en de naastgelegen randzones. Er werd geen verandering gevonden wanneer een controle contrastmiddel werd gegeven, of wanneer het specifieke contrastmiddel werd toegediend aan muizen zonder hartinfarct. De MRI resultaten werden bevestigd met fluorescentie microscopie, waarmee veel colokalisatie werd gevonden van het contrastmiddel met de microvaten in het infarctgebied en de randzones van het hart.

In Hoofdstuk 8 werd microvasculaire schade in de nieren als gevolg van ischemie en reperfusie onderzocht met behulp van kwantitatieve MRI. Deze ischemie-reperfusie schade speelt waarschijnlijk een belangrijke rol bij de ontwikkeling van nierfunctiestoornissen na een niertransplantatie. Tot 24 uur na ischemie-reperfusie werd een significante reductie in renale oxygenatie en functie waargenomen in een beschadigde nier in vergelijking met de andere, gezonde nier. Dit duidt mogelijk op voortdurende ontwikkeling van ischemische schade in de aangedane nier. 
In Hoofdstuk 9 worden de voorgaande resultaten in een breder perspectief geplaatst en wordt bediscussieerd welke uitdagingen overwonnen dienen te worden op weg naar de klinische toepassing van kwantitatieve contrastversterkte MRI van de microvasculatuur. 


\section{Dankwoord}

Als je snel wilt gaan moet je alleen gaan.

Als je ver wilt komen moet je samen gaan.

Afrikaans gezegde

Ja, het is gelukt, mijn proefschrift is af! Maar het boekje dat nu voor je ligt had ik natuurlijk nooit in mijn eentje kunnen schrijven. De afgelopen vier jaar heb ik zeer plezierig samengewerkt met meerdere groepen binnen het ziekenhuis en de universiteit, en ook buiten het werk hebben verschillende mensen indirect bijgedragen aan een leuke promotietijd in Maastricht. Een aantal mensen zou ik hieronder graag willen bedanken, maar bij deze dank ik ook alvast iedereen die ik onverhoopt vergeten ben te noemen.

Beste Walter, je deur stond altijd open en ik heb veel geleerd van onze discussies, je passie voor de wetenschap en je gedreven manier van werken. Bedankt voor alle kansen en uitdagingen die je me hebt gegeven, ik had me geen betere begeleider kunnen wensen.

Mark, als promotor was je nauw betrokken bij mijn onderzoek. Tijdens onze maandelijkse werkoverleggen kwam je vaak met interessante weetjes en ideeën voor nieuwe experimenten. Ik ben erg blij dat het cardiale hoofdstuk na veel tegenslag uiteindelijk toch nog zo goed is gelukt.

Met stip op drie: Kim. Mijn paranimf en partner in crime bij de meeste experimenten uit dit boekje. De vele dagen achter de scanner of in het donker achter de microscoop vond ik altijd erg gezellig, en met behulp van Wikipedia hebben we vele relevante, maar ook minstens net zo veel minder relevante vragen opgelost. Ik denk dat we de afgelopen jaren goede vrienden zijn geworden en hoop dat we dat ook in de toekomst zullen blijven.

Marc van Zandvoort, ook jij hartelijk dank voor je bijdrage aan de microscopie gedeeltes van dit boekje.

Tilman, je enthousiasme voor wetenschap en chemie werkt erg aanstekelijk. Dankzij jou en Dennis voelde ik me soms toch nog een beetje chemicus. Bedankt voor alle peptides en wedges, want zonder dat was dit boekje vast en zeker een stuk dunner geworden.

Een grote pluim gaat naar de biotechnici Allard, Niek, Hanneke (super dat je zelfs in je vakantie nog bereid was om me te helpen!) en Viviane. Jullie grote kennis en kunde in het uitvoeren van operaties op muizen waardeer ik enorm. Dankzij jullie verliepen de operaties (meestal) erg soepel en jullie hebben daardoor een onmisbare bijdrage geleverd aan dit proefschrift. Ik zou 
hierbij ook graag de medewerkers van de CPV willen bedanken voor de goede verzorging van mijn in totaal bijna 150 muizen.

Eva, dank je voor de gezellige samenwerking bij het nieren-project. Ik vond het erg leuk om eens aan een heel ander onderwerp te werken en ik ben blij dat het zulke mooie resultaten heeft opgeleverd.

Tijdens mijn promotie heb ik de kans gekregen om twee studenten te mogen begeleiden. Isabela en Martijn, hartelijk dank voor jullie inzet, ik vond het erg leuk om met jullie samen te werken. Veel succes met jullie eigen carrière!

Zeker niet onbelangrijk tijdens een promotie is een leuke groep collega's. Robbert-Jan, ik vond het altijd erg leuk om je kamergenoot te zijn en om soms op vrijdagmiddag een stukje mee te zingen met de radio. Bedankt ook voor de gezellig trip naar de ISMRM in Toronto! Frank, inhoudelijk konden we elkaar weinig helpen, maar wat betreft alle praktische zaken die een promotie met zich mee brengt juist des te meer. Ik vond het leuk om samen met jou en Robbert-Jan ongeveer hetzelfde traject te volgen en dank je natuurlijk voor de tip over de grootte van mijn captions! Karolien, bedankt dat je in de afgelopen vier jaar mijn collega, onderbuurvrouw en vriendin bent geworden. Ik ga onze gezellige avondjes thuis en jouw uitstekende kookkunst zeker missen, maar ik weet zeker dat we goede vriendinnen zullen blijven! Hartelijk dank ook aan Jos voor alle hulp bij de scanner, en aan Marc voor het oplossen van mijn soms toch wel merkwaardige computerproblemen. Verder zou ik graag alle oude en nieuwe collega's willen bedanken voor een leuke tijd bij de Radiologie: Suzanne, Jaap, Marc, Eline, Gerrit, Dave, Quido, Tim, Vera, Sanne, Max, Robbert, Nils, Bas, Maarten, Doenja, Monique, Robert, Stefan, Ghazal en Eline. Dank ook aan de collega's van de afdelingen Biofysica en Fysiologie voor de gezellige praatjes en algemene interesse als ik bij jullie op de afdeling een experiment kwam doen.

Een andere belangrijke groep zijn mijn vrienden van de Koninklijke Harmonie van Heer: Nicole, Dennis, Frank, Rianne, Joost, Olav, Jaap, Yvonne en Hilde. Dankzij jullie voelde ik me al snel helemaal thuis in Maastricht. Bedankt voor alle gezellige repetities, weekendjes weg en andere activiteiten! Nicole, ook onze wekelijkse squash-activiteiten (en natuurlijk het bijbehorende kletsen) ga ik zeker missen!

Remmelt, Grietje, Miranda en Maurice, ook jullie zou ik graag willen bedanken voor jullie oprechte interesse in mijn werk. Mees, jij snapt er nu nog wel niets van, maar als je boekjes lezen over een jaar of 10 nog steeds zo leuk vindt dan komt dat vast nog wel goed. 
Lieve pap, mam en Corien, jullie mogen hier zeker niet ontbreken. Zonder jullie onvoorwaardelijke steun, vertrouwen en advies was ik nooit zo ver gekomen als ik nu ben. Bedankt voor alles! Corien, geweldig dat je tijdens de verdediging naast me staat als paranimf. Ik vind het erg leuk dat ik nu weer dichterbij woon, nu kunnen we in elk geval weer wat vaker samen op stap.

Lieve Pieter, ik word altijd vrolijk als ik bij jou ben. Jouw bijdrage aan dit boekje is weliswaar niet inhoudelijk, maar is des te meer in hoe het er nu uit ziet. En laten we wel wezen, dat wordt uiteindelijk toch door meer mensen beoordeeld dan de inhoud. Dank je voor een geweldige vakantie naar Kenia waarvan nu een foto op de voorkant prijkt. Dank je ook dat je me af en toe wat met LATEX hebt geholpen, dat heeft ongetwijfeld veel Word-frustraties voorkomen. Dank je eigenlijk voor alles! 



\section{Scientific publications}

This thesis is based on the following publications:

- Oostendorp M, Post MJ and Backes WH. Vessel growth and function: depiction with contrast-enhanced MR imaging. Radiology. 2009; 251: 317-35.

- Oostendorp M, Douma K, Hackeng TM, Dirksen A, Post MJ, Van Zandvoort MAMJ and Backes WH. Quantitative molecular magnetic resonance imaging using cNGR-labeled paramagnetic quantum dots. Cancer Research. 2008; 68: 7676-83.

- Oostendorp M, Douma K, Hackeng TM, Post MJ, Van Zandvoort MAMJ and Backes WH. Gadolinium-labeled quantum dots for molecular magnetic resonance imaging: $R_{1}$ versus $R_{2}$ mapping. Magnetic Resonance in Medicine. 2009, provisionally accepted.

- Oostendorp M, Douma K, Hackeng TM, Post MJ, Van Zandvoort MAMJ and Backes WH. Pharmacokinetics of contrast agents targeted to the tumor vasculature in molecular magnetic resonance imaging. Contrast Media and Molecular Imaging. 2009, accepted.

- Oostendorp M, Douma K, Slaaf DW, Post MJ, Backes WH and Van Zandvoort MAMJ. Evaluation of magnetic resonance vessel size imaging by two-photon laser scanning microscopy. Magnetic Resonance in Medicine. 2009, accepted.

- Oostendorp M, Douma K, Wagenaar A, Slenter JMGM, Hackeng TM, Van Zandvoort MAMJ, Post MJ and Backes WH. Molecular MRI of myocardial angiogenesis after acute myocardial infarction. Circulation. 2009, provisionally accepted.

- Oostendorp M, De Vries EE, Slenter JMGM, Peutz-Kootstra C, Snoeijs MG, Post MJ, Van Heurn LWE and Backes WH. Magnetic resonance imaging of renal oxygenation and function after normothermic ischemiareperfusion injury. 2009, submitted. 
Other publications:

- Jaspers K, Aerts HJWL, Leiner T, Oostendorp M, Van Riel NAW, Post MJ and Backes WH. Reliability of pharmacokinetic parameters: small versus medium-sized contrast agents. Magnetic Resonance in Medicine. 2009; 62: 779-787.

- Engelke UHF, Oostendorp $\mathbf{M}$ and Wevers RA. NMR spectroscopy of body fluids as a metabolomics approach to inborn errors of metabolism. In: The handbook of metabonomics and metabolomics. Lindon JC, Nicholson JK and Holmes E. (Eds.), 1 ${ }^{\text {st }}$ Ed. Elsevier, 2007.

- Oostendorp M, Engelke UHF, Willemsen MAAP and Wevers RA. Diagnosing inborn errors of lipid metabolism using ${ }^{1} \mathrm{H}$ nuclear magnetic resonance spectroscopy. Clinical Chemistry. 2006; 52: 1395-1405.

- Walma T, Aelen JMA, Nabuurs SB, Van den Berk L, Oostendorp M, Hendriks W and Vuister GW. A closed binding pocket modifies the structure of an alternatively spliced form of the second PDZ domain of PTP-BL. Structure. 2004; 12: 11-20. 


\section{Curriculum vitae}

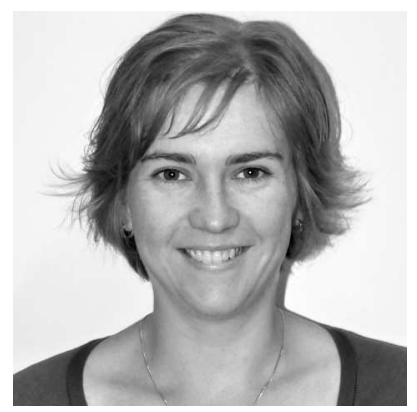

Marlies Oostendorp was born on October $11^{\text {th }} 1982$ in Arnhem, The Netherlands. In 2000, she finished her secondary school education (Gymnasium) at the Olympus College in Arnhem. In the same year, she started her Chemistry studies at the Radboud University Nijmegen, where she obtained her Master's degree cum laude in 2005. She did her major in Biophysical Chemistry at the department of Biophysical Chemistry of the Radboud University Nijmegen, under the supervision of Dr. Hugo van Ingen, Dr. Mark Hilge and Prof. Dr. Geerten W. Vuister. During this research project, she studied the structure, mobility, and function of two different protein domains using high resolution nuclear magnetic resonance (NMR) spectroscopy. Marlies did her minor in Clinical Chemistry at the Laboratory of Pediatrics and Neurology of the University Medical Center St. Radboud in Nijmegen, under the supervision of Dr. Udo H.F. Engelke and Prof. Dr. Ron A. Wevers. Here, she studied the applicability of NMR spectroscopy to identify and quantify lipids in the blood plasma of patients suffering from inborn errors in lipid or cholesterol metabolism. She was granted the Unilever research award for talented university graduates, for the work performed during both her major and minor research projects. In 2005, she started her PhD project at the department of Radiology of the Maastricht University Medical Center, under the supervision of Dr. Ir. Walter H. Backes and Prof. Dr. Mark J. Post, which resulted in the work described in this thesis. Marlies presented her work during several international conferences, for which she received travel grants from the International Society for Magnetic Resonance in Medicine, and from the Royal Dutch Academy of Sciences. In October 2009, Marlies started her Clinical Chemistry residency at the University Medical Center Utrecht. 


$$
\text { WERKVERSCHAFFING }
$$

NATUURLLIK

VERMELDT ELKE

ONDERZOEKER IN

ZIIN ONDERZOEK DAT

VERDER ONDERZOEK NOODZZAKELIJK IS

Loesje 